\title{
The Confrontation between General Relativity and Experiment
}

\author{
Clifford M. Will \\ Department of Physics \\ University of Florida \\ Gainesville FL 32611, U.S.A. \\ email: cmw@physics.ufl.edu \\ http://www.phys.ufl.edu/ cmw/
}

Accepted: 6 June 2014

Published: 11 June 2014

(Update of lrr-2006-3)

\begin{abstract}
The status of experimental tests of general relativity and of theoretical frameworks for analyzing them is reviewed and updated. Einstein's equivalence principle (EEP) is well supported by experiments such as the Eötvös experiment, tests of local Lorentz invariance and clock experiments. Ongoing tests of EEP and of the inverse square law are searching for new interactions arising from unification or quantum gravity. Tests of general relativity at the post-Newtonian level have reached high precision, including the light deflection, the Shapiro time delay, the perihelion advance of Mercury, the Nordtvedt effect in lunar motion, and frame-dragging. Gravitational wave damping has been detected in an amount that agrees with general relativity to better than half a percent using the Hulse-Taylor binary pulsar, and a growing family of other binary pulsar systems is yielding new tests, especially of strong-field effects. Current and future tests of relativity will center on strong gravity and gravitational waves.
\end{abstract}

Keywords: Tests of relativistic gravity, Theories of gravity, Post-Newtonian limit, Gravitational radiation

This review is licensed under a Creative Commons Attribution-Non-Commercial 3.0 Germany License. http://creativecommons .org/licenses/by-nc/3.0/de/ 


\section{Imprint / Terms of Use}

Living Reviews in Relativity is a peer reviewed open access journal published by the Max Planck Institute for Gravitational Physics, Am Mühlenberg 1, 14476 Potsdam, Germany. ISSN 1433-8351.

This review is licensed under a Creative Commons Attribution-Non-Commercial 3.0 Germany License: http://creativecommons.org/licenses/by-nc/3.0/de/. Figures that have been previously published elsewhere may not be reproduced without consent of the original copyright holders.

Because a Living Reviews article can evolve over time, we recommend to cite the article as follows:

Clifford M. Will,

"The Confrontation between General Relativity and Experiment", Living Rev. Relativity, 17, (2014), 4. URL (accessed <date>): http://www.livingreviews.org/lrr-2014-4

The date given as $<$ date $>$ then uniquely identifies the version of the article you are referring to. 


\section{Article Revisions}

Living Reviews supports two ways of keeping its articles up-to-date:

Fast-track revision. A fast-track revision provides the author with the opportunity to add short notices of current research results, trends and developments, or important publications to the article. A fast-track revision is refereed by the responsible subject editor. If an article has undergone a fast-track revision, a summary of changes will be listed here.

Major update. A major update will include substantial changes and additions and is subject to full external refereeing. It is published with a new publication number.

For detailed documentation of an article's evolution, please refer to the history document of the article's online version at http://www. livingreviews.org/lrr-2014-4.

11 June 2014: Major revision, updated and expanded.

Added new Section 2.3.3 on the Pioneer anomaly; split former Section 3 into new 3 and 4, and extended Section 3.3 on competing theories of gravity; added new Sections 5.3 and 5.4 on compact binary systems; added a new Section 8 on astrophysical and cosmological tests.

The number of references increased from 299 to 454. Added two figures (8, 9) and updated Figures 1, 3, 5, and 7 . 


\section{Contents}

1 Introduction $\quad 7$

2 Tests of the Foundations of Gravitation Theory 9

2.1 The Einstein equivalence principle . . . . . . . . . . . . . . . . . . . 9

2.1.1 Tests of the weak equivalence principle . . . . . . . . . . . . . . . 10

2.1 .2 Tests of local Lorentz invariance . . . . . . . . . . . . . . . . . . . . . . . . . . . . . . . . . . . . . .

2.1.3 Tests of local position invariance . . . . . . . . . . . . . . . . . . . . . . . . . . . . . . . . . . . .

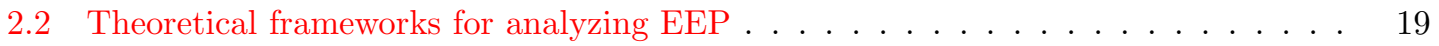

2.2.1 Schiff's conjecture . . . . . . . . . . . . . . . . . . . . . . . . . . . . . . . . . . . .

2.2 .2 The $T H \epsilon \mu$ formalism . . . . . . . . . . . . . . . . . . . . 20

2.2 .3 The $c^{2}$ formalism . . . . . . . . . . . . . . . . . 22

2.2.4 The standard model extension (SME) . . . . . . . . . . . . . . . . . 24

2.3 EEP, particle physics, and the search for new interactions . . . . . . . . . . . . 25

2.3.1 The "fifth" force . . . . . . . . . . . . . . . . . . 26

2.3.2 Short-range modifications of Newtonian gravity . . . . . . . . . . . . . 27

2.3.3 The Pioneer anomaly . . . . . . . . . . . . . . . . 27

3 Metric Theories of Gravity and the PPN Formalism 28

3.1 Metric theories of gravity and the strong equivalence principle . . . . . . . . . . 28

3.1.1 Universal coupling and the metric postulates . . . . . . . . . . . . . . 28

3.1.2 The strong equivalence principle . . . . . . . . . . . . . . . . . . . . 28

3.2 The parametrized post-Newtonian formalism . . . . . . . . . . . . . . . 30

3.3 Competing theories of gravity . . . . . . . . . . . . . . . . . . . . . . . . . . . . . . . . 34

3.3 .1 General relativity . . . . . . . . . . . . . . . . . . . . . . . . 34

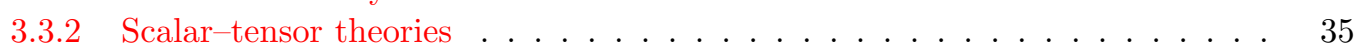

$3.3 .3 f(R)$ theories . . . . . . . . . . . . . . . . . 37

3.3 .4 Vector-tensor theories . . . . . . . . . . . . . . . . 37

3.3.5 Tensor-vector-scalar $(\mathrm{TeVeS})$ theories . . . . . . . . . . . . . . . . . . 39

3.3.6 Quadratic gravity and Chern-Simons theories . . . . . . . . . . . . 40

3.3.7 Massive gravity . . . . . . . . . . . . . . . . . . . 41

4 Tests of Post-Newtonian Gravity $\quad 42$

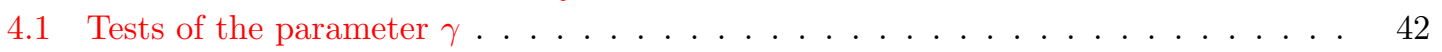

4.1 The deflection of light . . . . . . . . . . . . . . . . . . . . . . . . 42

4.1 .2 The time delay of light . . . . . . . . . . . . . . . . . . . . 44

4.1.3 Shapiro time delay and the speed of gravity . . . . . . . . . . . . . . . . . . . 45

4.2 The perihelion shift of Mercury . . . . . . . . . . . . . . . . . . . 46

4.3 Tests of the strong equivalence principle . . . . . . . . . . . . . . . . . . . . 47

4.3.1 The Nordtvedt effect and the lunar Eötvös experiment . . . . . . . . . . . . 47

4.3.2 Preferred-frame and preferred-location effects . . . . . . . . . . . . . . 49

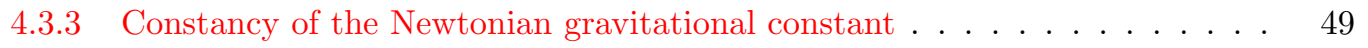

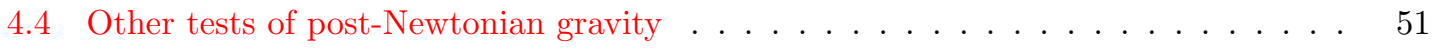

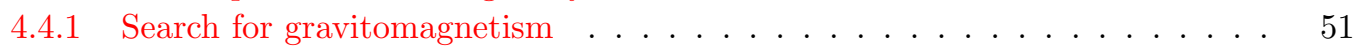

4.4 Geodetic precession . . . . . . . . . . . . . . . . . . 52

4.4 .3 Tests of post-Newtonian conservation laws . . . . . . . . . . . . . . . . . 53

4.5 Prospects for improved PPN parameter values . . . . . . . . . . . . . . 54 
5 Strong Gravity and Gravitational Waves: Tests for the 21st Century 5

5.1 Strong-field systems in general relativity . . . . . . . . . . . . . . . 55

5.1 Defining weak and strong gravity . . . . . . . . . . . . . . . . 55

5.1 .2 Compact bodies and the strong equivalence principle . . . . . . . . . 56

5.2 Motion and gravitational radiation in general relativity: A history . . . . . . . . 57

5.3 Compact binary systems in general relativity . . . . . . . . . . . . . . . . . . 59

5.3 .1 Einstein's equations in "relaxed" form . . . . . . . . . . . . . . 59

5.3.2 Equations of motion and gravitational waveform . . . . . . . . . . 61

5.4 Compact binary systems in scalar-tensor theories . . . . . . . . . . . . . . . 62

5.4.1 Scalar-tensor equations in "relaxed" form . . . . . . . . . . . . . 63

5.4 .2 Equations of motion and gravitational waveform . . . . . . . . . . . . 64

5.4 .3 Binary systems containing black holes . . . . . . . . . . . . . . 66

6 Stellar System Tests of Gravitational Theory $\quad 68$

6.1 The binary pulsar and general relativity . . . . . . . . . . . . . . . 68

6.2 A zoo of binary pulsars . . . . . . . . . . . . . . . . . . . . . . 72

6.3 Binary pulsars and alternative theories . . . . . . . . . . . . . . . . . . . . . . . 74

6.4 Binary pulsars and scalar-tensor gravity . . . . . . . . . . . . . . . 75

7 Gravitational-Wave Tests of Gravitational Theory $\quad 79$

7.1 Gravitational-wave observatories . . . . . . . . . . . . . . . . . . 79

7.2 Gravitational-wave amplitude and polarization . . . . . . . . . . . . . . 79

7.2 .1 General relativity . . . . . . . . . . . . . . . . . . 79

7.2 .2 Alternative theories of gravity . . . . . . . . . . . . . . . . . . . 80

7.3 Gravitational-wave phase evolution . . . . . . . . . . . . . . . . 83

7.3.1 General relativity . . . . . . . . . . . . . . . . . . . . 83

7.3.2 Alternative theories of gravity . . . . . . . . . . . . . . . . 84

7.4 Speed of gravitational waves . . . . . . . . . . . . . . . . . . 85

8 Astrophysical and Cosmological Tests $\quad 87$

9 Conclusions $\quad 88$

$\begin{array}{lr}\text { References } & 90\end{array}$

\section{List of Tables}

1 Bounds on cosmological variation of fundamental constants of non-gravitational

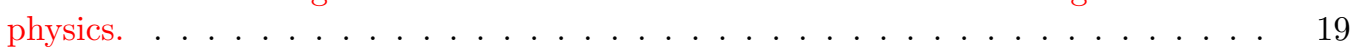

2 The PPN Parameters and their significance. . . . . . . . . . . . . . . . . . . . . . . . . 31

3 Metric theories and their PPN parameter values. . . . . . . . . . . . . . . 35

4 Current limits on the PPN parameters. . . . . . . . . . . . . . . . . . . . . 46

5 Constancy of the gravitational constant. . . . . . . . . . . . . . . . . 50

6 Parameters used in the equations of motion. . . . . . . . . . . . . . . . . . . . 64

7 Parameters of the binary pulsar B1913+16. . . . . . . . . . . . . . . . 68

8 Parameters of other binary pulsars. . . . . . . . . . . . . . . . . . 74 



\section{Introduction}

When general relativity was born 100 years ago, experimental confirmation was almost a side issue. Admittedly, Einstein did calculate observable effects of general relativity, such as the perihelion advance of Mercury, which he knew to be an unsolved problem, and the deflection of light, which was subsequently verified. But compared to the inner consistency and elegance of the theory, he regarded such empirical questions as almost secondary. He famously stated that if the measurements of light deflection disagreed with the theory he would "feel sorry for the dear Lord, for the theory is correct!".

By contrast, today experimental gravitation is a major component of the field, characterized by continuing efforts to test the theory's predictions, both in the solar system and in the astronomical world, to detect gravitational waves from astronomical sources, and to search for possible gravitational imprints of phenomena originating in the quantum, high-energy or cosmological realms.

The modern history of experimental relativity can be divided roughly into four periods: Genesis, Hibernation, a Golden Era, and the Quest for Strong Gravity. The Genesis (1887-1919) comprises the period of the two great experiments which were the foundation of relativistic physics - the Michelson-Morley experiment and the Eötvös experiment - and the two immediate confirmations of general relativity - the deflection of light and the perihelion advance of Mercury. Following this was a period of Hibernation (1920-1960) during which theoretical work temporarily outstripped technology and experimental possibilities, and, as a consequence, the field stagnated and was relegated to the backwaters of physics and astronomy.

But beginning around 1960, astronomical discoveries (quasars, pulsars, cosmic background radiation) and new experiments pushed general relativity to the forefront. Experimental gravitation experienced a Golden Era (1960-1980) during which a systematic, world-wide effort took place to understand the observable predictions of general relativity, to compare and contrast them with the predictions of alternative theories of gravity, and to perform new experiments to test them. New technologies - atomic clocks, radar and laser ranging, space probes, cryogenic capabilities, to mention only a few - played a central role in this golden era. The period began with an experiment to confirm the gravitational frequency shift of light (1960) and ended with the reported decrease in the orbital period of the Hulse-Taylor binary pulsar at a rate consistent with the general relativistic prediction of gravitational-wave energy loss (1979). The results all supported general relativity, and most alternative theories of gravity fell by the wayside (for a popular review, see [421]).

Since that time, the field has entered what might be termed a Quest for Strong Gravity. Much like modern art, the term "strong" means different things to different people. To one steeped in general relativity, the principal figure of merit that distinguishes strong from weak gravity is the quantity $\epsilon \sim G M / R c^{2}$, where $G$ is the Newtonian gravitational constant, $M$ is the characteristic mass scale of the phenomenon, $R$ is the characteristic distance scale, and $c$ is the speed of light. Near the event horizon of a non-rotating black hole, or for the expanding observable universe, $\epsilon \sim 1$; for neutron stars, $\epsilon \sim 0.2$. These are the regimes of strong gravity. For the solar system, $\epsilon<10^{-5}$; this is the regime of weak gravity.

An alternative view of "strong" gravity comes from the world of particle physics. Here the figure of merit is $G M / R^{3} c^{2} \sim \ell^{-2}$, where the Riemann curvature of spacetime associated with the phenomenon, represented by the left-hand-side, is comparable to the inverse square of a favorite length scale $\ell$. If $\ell$ is the Planck length, this would correspond to the regime where one expects conventional quantum gravity effects to come into play. Another possible scale for $\ell$ is the $\mathrm{TeV}$ scale associated with many models for unification of the forces, or models with extra spacetime dimensions. From this viewpoint, strong gravity is where the curvature is comparable to the inverse length squared. Weak gravity is where the curvature is much smaller than this. The universe at the Planck time is strong gravity. Just outside the event horizon of an astrophysical black hole is weak gravity. 
Considerations of the possibilities for new physics from either point of view have led to a wide range of questions that will motivate new tests of general relativity as we move into its second century:

- Are the black holes that are in evidence throughout the universe truly the black holes of general relativity?

- Do gravitational waves propagate with the speed of light and do they contain more than the two basic polarization states of general relativity?

- Does general relativity hold on cosmological distance scales?

- Is Lorentz invariance strictly valid, or could it be violated at some detectable level?

- Does the principle of equivalence break down at some level?

- Are there testable effects arising from the quantization of gravity?

In this update of our Living Review, we will summarize the current status of experiments, and attempt to chart the future of the subject. We will not provide complete references to early work done in this field but instead will refer the reader to selected recent papers and to the appropriate review articles and monographs, specifically to Theory and Experiment in Gravitational Physics [420], hereafter referred to as TEGP; references to TEGP will be by chapter or section, e.g., "TEGP 8.9". Additional reviews in this subject are [40, 361, 392]. The "Resource Letter" by the author [428], contains an annotated list of 100 key references for experimental gravity. 


\section{Tests of the Foundations of Gravitation Theory}

\subsection{The Einstein equivalence principle}

The principle of equivalence has historically played an important role in the development of gravitation theory. Newton regarded this principle as such a cornerstone of mechanics that he devoted the opening paragraph of the Principia to it. In 1907, Einstein used the principle as a basic element in his development of general relativity (GR). We now regard the principle of equivalence as the foundation, not of Newtonian gravity or of GR, but of the broader idea that spacetime is curved. Much of this viewpoint can be traced back to Robert Dicke, who contributed crucial ideas about the foundations of gravitation theory between 1960 and 1965. These ideas were summarized in his influential Les Houches lectures of 1964 [130], and resulted in what has come to be called the Einstein equivalence principle (EEP).

One elementary equivalence principle is the kind Newton had in mind when he stated that the property of a body called "mass" is proportional to the "weight", and is known as the weak equivalence principle (WEP). An alternative statement of WEP is that the trajectory of a freely falling "test" body (one not acted upon by such forces as electromagnetism and too small to be affected by tidal gravitational forces) is independent of its internal structure and composition. In the simplest case of dropping two different bodies in a gravitational field, WEP states that the bodies fall with the same acceleration (this is often termed the Universality of Free Fall, or UFF).

The Einstein equivalence principle (EEP) is a more powerful and far-reaching concept; it states that:

1. WEP is valid.

2. The outcome of any local non-gravitational experiment is independent of the velocity of the freely-falling reference frame in which it is performed.

3. The outcome of any local non-gravitational experiment is independent of where and when in the universe it is performed.

The second piece of EEP is called local Lorentz invariance (LLI), and the third piece is called local position invariance (LPI).

For example, a measurement of the electric force between two charged bodies is a local nongravitational experiment; a measurement of the gravitational force between two bodies (Cavendish experiment) is not.

The Einstein equivalence principle is the heart and soul of gravitational theory, for it is possible to argue convincingly that if EEP is valid, then gravitation must be a "curved spacetime" phenomenon, in other words, the effects of gravity must be equivalent to the effects of living in a curved spacetime. As a consequence of this argument, the only theories of gravity that can fully embody EEP are those that satisfy the postulates of "metric theories of gravity", which are:

1. Spacetime is endowed with a symmetric metric.

2. The trajectories of freely falling test bodies are geodesics of that metric.

3. In local freely falling reference frames, the non-gravitational laws of physics are those written in the language of special relativity.

The argument that leads to this conclusion simply notes that, if EEP is valid, then in local freely falling frames, the laws governing experiments must be independent of the velocity of the frame (local Lorentz invariance), with constant values for the various atomic constants (in order to be independent of location). The only laws we know of that fulfill this are those that are compatible with special relativity, such as Maxwell's equations of electromagnetism, and the standard model of 
particle physics. Furthermore, in local freely falling frames, test bodies appear to be unaccelerated, in other words they move on straight lines; but such "locally straight" lines simply correspond to "geodesics" in a curved spacetime (TEGP 2.3 [420]).

General relativity is a metric theory of gravity, but then so are many others, including the Brans-Dicke theory and its generalizations. Theories in which varying non-gravitational constants are associated with dynamical fields that couple to matter directly are not metric theories. Neither, in this narrow sense, is superstring theory (see Section 2.3), which, while based fundamentally on a spacetime metric, introduces additional fields (dilatons, moduli) that can couple to material stressenergy in a way that can lead to violations, say, of WEP. It is important to point out, however, that there is some ambiguity in whether one treats such fields as EEP-violating gravitational fields, or simply as additional matter fields, like those that carry electromagnetism or the weak interactions. Still, the notion of curved spacetime is a very general and fundamental one, and therefore it is important to test the various aspects of the Einstein equivalence principle thoroughly. We first survey the experimental tests, and describe some of the theoretical formalisms that have been developed to interpret them. For other reviews of EEP and its experimental and theoretical significance, see [183, 239]; for a pedagogical review of the variety of equivalence principles, see [128].

\subsubsection{Tests of the weak equivalence principle}

A direct test of WEP is the comparison of the acceleration of two laboratory-sized bodies of different composition in an external gravitational field. If the principle were violated, then the accelerations of different bodies would differ. The simplest way to quantify such possible violations of WEP in a form suitable for comparison with experiment is to suppose that for a body with inertial mass $m_{\mathrm{I}}$, the passive gravitational mass $m_{\mathrm{P}}$ is no longer equal to $m_{\mathrm{I}}$, so that in a gravitational field $g$, the acceleration is given by $m_{\mathrm{I}} a=m_{\mathrm{P}} g$. Now the inertial mass of a typical laboratory body is made up of several types of mass-energy: rest energy, electromagnetic energy, weak-interaction energy, and so on. If one of these forms of energy contributes to $m_{\mathrm{P}}$ differently than it does to $m_{\mathrm{I}}$, a violation of WEP would result. One could then write

$$
m_{\mathrm{P}}=m_{\mathrm{I}}+\sum_{A} \frac{\eta^{A} E^{A}}{c^{2}},
$$

where $E^{A}$ is the internal energy of the body generated by interaction $A, \eta^{A}$ is a dimensionless parameter that measures the strength of the violation of WEP induced by that interaction, and $c$ is the speed of light. A measurement or limit on the fractional difference in acceleration between two bodies then yields a quantity called the "Eötvös ratio" given by

$$
\eta \equiv 2 \frac{\left|a_{1}-a_{2}\right|}{\left|a_{1}+a_{2}\right|}=\sum_{A} \eta^{A}\left(\frac{E_{1}^{A}}{m_{1} c^{2}}-\frac{E_{2}^{A}}{m_{2} c^{2}}\right),
$$

where we drop the subscript "I" from the inertial masses. Thus, experimental limits on $\eta$ place limits on the WEP-violation parameters $\eta^{A}$.

Many high-precision Eötvös-type experiments have been performed, from the pendulum experiments of Newton, Bessel, and Potter to the classic torsion-balance measurements of Eötvös [148], Dicke [131], Braginsky [65], and their collaborators (for a bibliography of experiments up to 1991, see [155]). . In the modern torsion-balance experiments, two objects of different composition are connected by a rod or placed on a tray and suspended in a horizontal orientation by a fine wire. If the gravitational acceleration of the bodies differs, and this difference has a component perpendicular to the suspension wire, there will be a torque induced on the wire, related to the angle between the wire and the direction of the gravitational acceleration $\boldsymbol{g}$. If the entire apparatus is rotated about some direction with angular velocity $\omega$, the torque will be modulated with period 


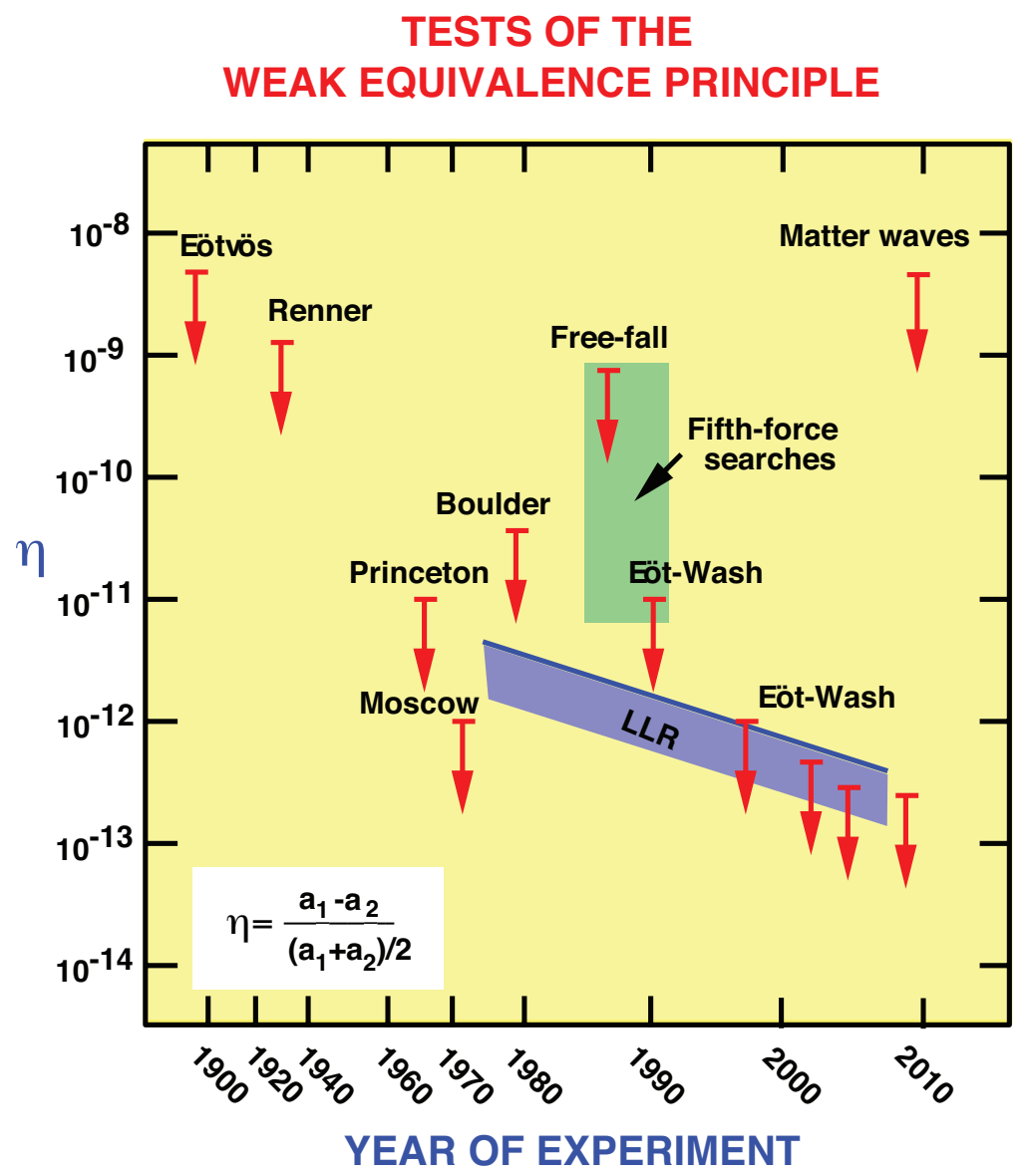

Figure 1: Selected tests of the weak equivalence principle, showing bounds on $\eta$, which measures fractional difference in acceleration of different materials or bodies. The free-fall and Eöt-Wash experiments were originally performed to search for a fifth force (green region, representing many experiments). The blue band shows evolving bounds on $\eta$ for gravitating bodies from lunar laser ranging (LLR).

$2 \pi / \omega$. In the experiments of Eötvös and his collaborators, the wire and $\boldsymbol{g}$ were not quite parallel because of the centripetal acceleration on the apparatus due to the Earth's rotation; the apparatus was rotated about the direction of the wire. In the Dicke and Braginsky experiments, $\boldsymbol{g}$ was that of the Sun, and the rotation of the Earth provided the modulation of the torque at a period of $24 \mathrm{hr}$ (TEGP 2.4 (a) [420]). Beginning in the late 1980s, numerous experiments were carried out primarily to search for a "fifth force" (see Section 2.3.1), but their null results also constituted tests of WEP. In the "free-fall Galileo experiment" performed at the University of Colorado, the relative free-fall acceleration of two bodies made of uranium and copper was measured using a laser interferometric technique. The "Eöt-Wash" experiments carried out at the University of Washington used a sophisticated torsion balance tray to compare the accelerations of various materials toward local topography on Earth, movable laboratory masses, the Sun and the galaxy [379, 29], and have reached levels of $2 \times 10^{-13}$ [1,354,402]. The resulting upper limits on $\eta$ are summarized in Figure 1.

The recent development of atom interferometry has yielded tests of WEP, albeit to modest accuracy, comparable to that of the original Eötvös experiment. In these experiments, one measures 
the local acceleration of the two separated wavefunctions of an atom such as Cesium by studying the interference pattern when the wavefunctions are combined, and compares that with the acceleration of a nearby macroscopic object of different composition [278, 294]. A claim that these experiments test the gravitational redshift [294] was subsequently shown to be incorrect [439].

A number of projects are in the development or planning stage to push the bounds on $\eta$ even lower. The project MICROSCOPE is designed to test WEP to $10^{-15}$. It is being developed by the French space agency CNES for launch in late 2015, for a one-year mission [280]. The drag-compensated satellite will be in a Sun-synchronous polar orbit at $700 \mathrm{~km}$ altitude, with a payload consisting of two differential accelerometers, one with elements made of the same material (platinum), and another with elements made of different materials (platinum and titanium). Other concepts for future improvements include advanced space experiments (Galileo-Galilei, STEP, STEQUEST), experiments on sub-orbital rockets, lunar laser ranging (see Section 4.3.1), binary pulsar observations, and experiments with anti-hydrogen. For an update on past and future tests of WEP, see the series of articles introduced by [372]. The recent discovery of a pulsar in orbit with two white-dwarf companions [332] may provide interesting new tests of WEP, because of the strong difference in composition between the neutron star and the white dwarfs, as well as precise tests of the Nordtvedt effect (see Section 4.3.1).

\subsubsection{Tests of local Lorentz invariance}

Although special relativity itself never benefited from the kind of "crucial" experiments, such as the perihelion advance of Mercury and the deflection of light, that contributed so much to the initial acceptance of GR and to the fame of Einstein, the steady accumulation of experimental support, together with the successful merger of special relativity with quantum mechanics, led to its acceptance by mainstream physicists by the late 1920s, ultimately to become part of the standard toolkit of every working physicist. This accumulation included

- the classic Michelson-Morley experiment and its descendents [279, 357, 208, 69],

- the Ives-Stillwell, Rossi-Hall, and other tests of time-dilation [200, 343, 151],

- tests of whether the speed of light is independent of the velocity of the source, using both binary X-ray stellar sources and high-energy pions [67, 8],

- tests of the isotropy of the speed of light [75, 340, 234].

In addition to these direct experiments, there was the Dirac equation of quantum mechanics and its prediction of anti-particles and spin; later would come the stunningly successful relativistic theory of quantum electrodynamics. For a pedagogical review on the occasion of the 2005 centenary of special relativity, see [426].

In 2015, on the 110th anniversary of the introduction of special relativity, one might ask "what is there to test?" Special relativity has been so thoroughly integrated into the fabric of modern physics that its validity is rarely challenged, except by cranks and crackpots. It is ironic then, that during the past several years, a vigorous theoretical and experimental effort has been launched, on an international scale, to find violations of special relativity. The motivation for this effort is not a desire to repudiate Einstein, but to look for evidence of new physics "beyond" Einstein, such as apparent, or "effective" violations of Lorentz invariance that might result from certain models of quantum gravity. Quantum gravity asserts that there is a fundamental length scale given by the Planck length, $\ell_{\mathrm{Pl}}=\left(\hbar G / c^{3}\right)^{1 / 2}=1.6 \times 10^{-33} \mathrm{~cm}$, but since length is not an invariant quantity (Lorentz-FitzGerald contraction), then there could be a violation of Lorentz invariance at some level in quantum gravity. In brane-world scenarios, while physics may be locally Lorentz invariant in the higher dimensional world, the confinement of the interactions of normal physics to our fourdimensional "brane" could induce apparent Lorentz violating effects. And in models such as string 
theory, the presence of additional scalar, vector, and tensor long-range fields that couple to matter of the standard model could induce effective violations of Lorentz symmetry. These and other ideas have motivated a serious reconsideration of how to test Lorentz invariance with better precision and in new ways.

A simple and useful way of interpreting some of these modern experiments, called the $c^{2}$ formalism, is to suppose that the electromagnetic interactions suffer a slight violation of Lorentz invariance, through a change in the speed of electromagnetic radiation $c$ relative to the limiting speed of material test particles ( $c_{0}$, made to take the value unity via a choice of units), in other words, $c \neq 1$ (see Section 2.2.3). Such a violation necessarily selects a preferred universal rest frame, presumably that of the cosmic background radiation, through which we are moving at about $370 \mathrm{~km} \mathrm{~s}^{-1}$ [253]. Such a Lorentz-non-invariant electromagnetic interaction would cause shifts in the energy levels of atoms and nuclei that depend on the orientation of the quantization axis of the state relative to our universal velocity vector, and on the quantum numbers of the state. The presence or absence of such energy shifts can be examined by measuring the energy of one such state relative to another state that is either unaffected or is affected differently by the supposed violation. One way is to look for a shifting of the energy levels of states that are ordinarily equally spaced, such as the Zeeman-split $2 J+1$ ground states of a nucleus of total spin $J$ in a magnetic field; another is to compare the levels of a complex nucleus with the atomic hyperfine levels of a hydrogen maser clock. The magnitude of these "clock anisotropies" turns out to be proportional to $\delta \equiv\left|c^{-2}-1\right|$.

The earliest clock anisotropy experiments were the Hughes-Drever experiments, performed in the period 1959-60 independently by Hughes and collaborators at Yale University, and by Drever at Glasgow University, although their original motivation was somewhat different $[194,136]$. The Hughes-Drever experiments yielded extremely accurate results, quoted as limits on the parameter $\delta \equiv c^{-2}-1$ in Figure 2. Dramatic improvements were made in the 1980s using laser-cooled trapped atoms and ions [325, 240, 81]. This technique made it possible to reduce the broading of resonance lines caused by collisions, leading to improved bounds on $\delta$ shown in Figure 2 (experiments labelled NIST, U. Washington and Harvard, respectively).

Also included for comparison is the corresponding limit obtained from Michelson-Morley type experiments (for a review, see [184]). In those experiments, when viewed from the preferred frame, the speed of light down the two arms of the moving interferometer is $c$, while it can be shown using the electrodynamics of the $c^{2}$ formalism, that the compensating Lorentz-FitzGerald contraction of the parallel arm is governed by the speed $c_{0}=1$. Thus the Michelson-Morley experiment and its descendants also measure the coefficient $c^{-2}-1$. One of these is the Brillet-Hall experiment [69], which used a Fabry-Pérot laser interferometer. In a recent series of experiments, the frequencies of electromagnetic cavity oscillators in various orientations were compared with each other or with atomic clocks as a function of the orientation of the laboratory [438, 254, 293, 20, 376]. These placed bounds on $c^{-2}-1$ at the level of better than a part in $10^{9}$. Haugan and Lämmerzahl [182] have considered the bounds that Michelson-Morley type experiments could place on a modified electrodynamics involving a "vector-valued" effective photon mass.

The $c^{2}$ framework focuses exclusively on classical electrodynamics. It has recently been extended to the entire standard model of particle physics by Kostelecký and colleagues [92, 93, 228]. The "standard model extension" (SME) has a large number of Lorentz-violating parameters, opening up many new opportunities for experimental tests (see Section 2.2.4). A variety of clock anisotropy experiments have been carried out to bound the electromagnetic parameters of the SME framework [227]. For example, the cavity experiments described above [438, 254, 293] placed bounds on the coefficients of the tensors $\tilde{\kappa}_{\mathrm{e}-}$ and $\tilde{\kappa}_{\mathrm{o}}$ (see Section 2.2.4 for definitions) at the levels of $10^{-14}$ and $10^{-10}$, respectively. Direct comparisons between atomic clocks based on different nuclear species place bounds on SME parameters in the neutron and proton sectors, depending on the nature of the transitions involved. The bounds achieved range from $10^{-27}$ to $10^{-32} \mathrm{GeV}$. 


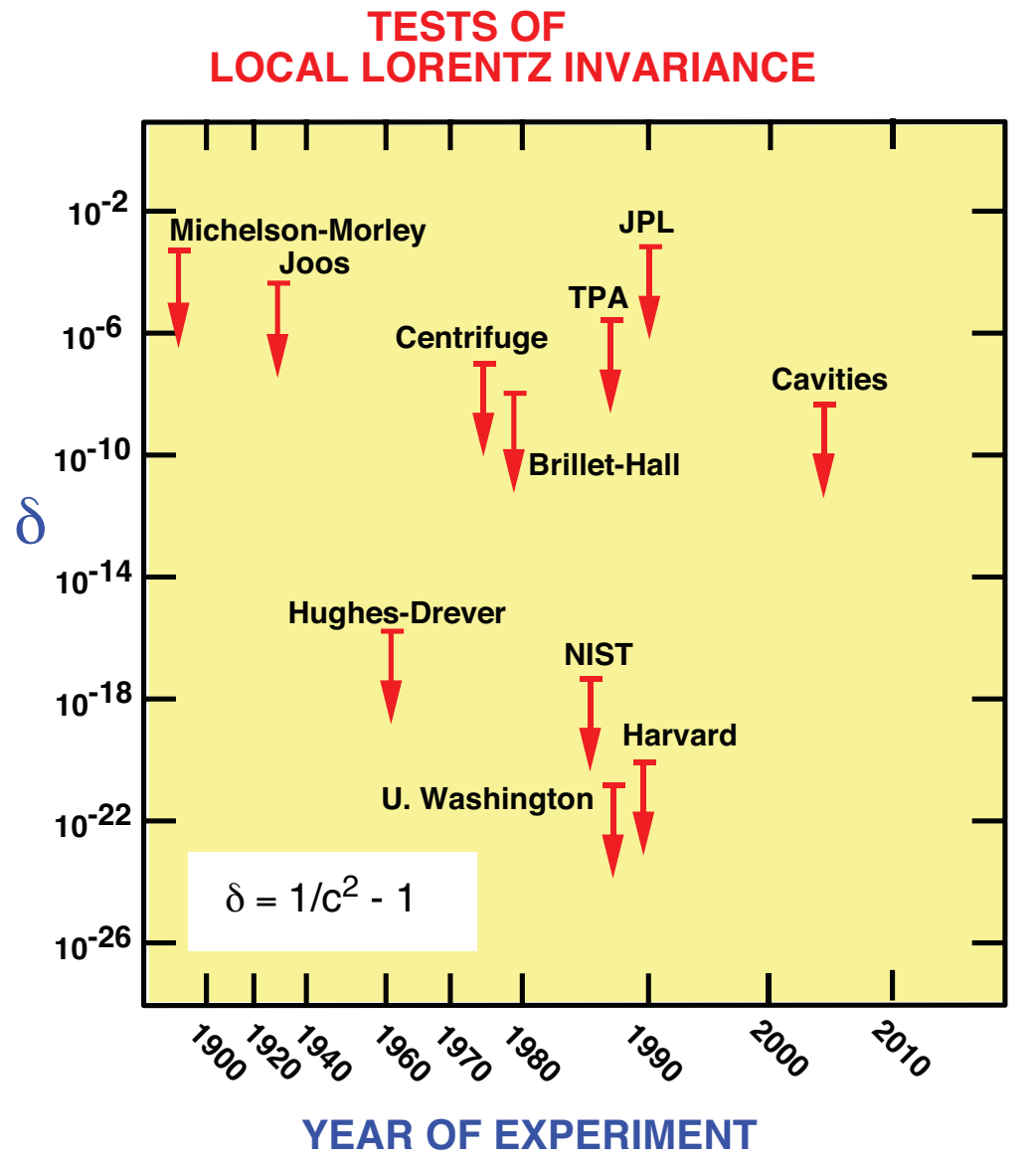

Figure 2: Selected tests of local Lorentz invariance showing the bounds on the parameter $\delta$, which measures the degree of violation of Lorentz invariance in electromagnetism. The Michelson-Morley, Joos, Brillet-Hall and cavity experiments test the isotropy of the round-trip speed of light. The centrifuge, twophoton absorption (TPA) and JPL experiments test the isotropy of light speed using one-way propagation. The most precise experiments test isotropy of atomic energy levels. The limits assume a speed of Earth of $370 \mathrm{~km} \mathrm{~s}^{-1}$ relative to the mean rest frame of the universe.

Recent examples include [440, 369].

Astrophysical observations have also been used to bound Lorentz violations. For example, if photons satisfy the Lorentz violating dispersion relation

$$
E^{2}=p^{2} c^{2}+E_{\mathrm{Pl}} f^{(1)}|p| c+f^{(2)} p^{2} c^{2}+\frac{f^{(3)}}{E_{\mathrm{Pl}}}|p|^{3} c^{3}+\ldots,
$$

where $E_{\mathrm{Pl}}=\left(\hbar c^{5} / G\right)^{1 / 2}$ is the Planck energy, then the speed of light $v_{\gamma}=\partial E / \partial p$ would be given, to linear order in the $f^{(n)}$ by

$$
\frac{v_{\gamma}}{c} \approx 1+\sum_{n \geq 1} \frac{(n-1) f_{\gamma}^{(n)} E^{n-2}}{2 E_{\mathrm{Pl}}^{n-2}}
$$

Such a Lorentz-violating dispersion relation could be a relic of quantum gravity, for instance. By bounding the difference in arrival time of high-energy photons from a burst source at large dis- 
tances, one could bound contributions to the dispersion for $n>2$. One limit, $\left|f^{(3)}\right|<128$ comes from observations of 1 and $2 \mathrm{TeV}$ gamma rays from the blazar Markarian 421 [48]. Another limit comes from birefringence in photon propagation: In many Lorentz violating models, different photon polarizations may propagate with different speeds, causing the plane of polarization of a wave to rotate. If the frequency dependence of this rotation has a dispersion relation similar to Eq. (3), then by studying "polarization diffusion" of light from a polarized source in a given bandwidth, one can effectively place a bound $\left|f^{(3)}\right|<10^{-4}$ [173]. Measurements of the spectrum of ultra-highenergy cosmic rays using data from the HiRes and Pierre Auger observatories show no evidence for violations of Lorentz invariance [378, 47]. Other testable effects of Lorentz invariance violation include threshold effects in particle reactions, gravitational Cerenkov radiation, and neutrino oscillations.

For thorough and up-to-date surveys of both the theoretical frameworks and the experimental results for tests of LLI see the reviews by Mattingly [273], Liberati [251] and Kostelecký and Russell [229]. The last article gives "data tables" showing experimental bounds on all the various parameters of the SME.

Local Lorentz invariance can also be violated in gravitational interactions; these will be discussed under the rubric of "preferred-frame effects" in Section 4.3.2.

\subsubsection{Tests of local position invariance}

The principle of local position invariance, the third part of EEP, can be tested by the gravitational redshift experiment, the first experimental test of gravitation proposed by Einstein. Despite the fact that Einstein regarded this as a crucial test of GR, we now realize that it does not distinguish between GR and any other metric theory of gravity, but is only a test of EEP. The iconic gravitational redshift experiment measures the frequency or wavelength shift $Z \equiv \Delta \nu / \nu=-\Delta \lambda / \lambda$ between two identical frequency standards (clocks) placed at rest at different heights in a static gravitational field. If the frequency of a given type of atomic clock is the same when measured in a local, momentarily co-moving freely falling frame (Lorentz frame), independent of the location or velocity of that frame, then the comparison of frequencies of two clocks at rest at different locations boils down to a comparison of the velocities of two local Lorentz frames, one at rest with respect to one clock at the moment of emission of its signal, the other at rest with respect to the other clock at the moment of reception of the signal. The frequency shift is then a consequence of the first-order Doppler shift between the frames. The structure of the clock plays no role whatsoever. The result is a shift

$$
Z=\frac{\Delta U}{c^{2}},
$$

where $\Delta U$ is the difference in the Newtonian gravitational potential between the receiver and the emitter. If LPI is not valid, then it turns out that the shift can be written

$$
Z=(1+\alpha) \frac{\Delta U}{c^{2}},
$$

where the parameter $\alpha$ may depend upon the nature of the clock whose shift is being measured (see TEGP 2.4 (c) [420] for details).

The first successful, high-precision redshift measurement was the series of Pound-Rebka-Snider experiments of $1960-1965$ that measured the frequency shift of gamma-ray photons from ${ }^{57} \mathrm{Fe}$ as they ascended or descended the Jefferson Physical Laboratory tower at Harvard University. The high accuracy achieved - one percent - was obtained by making use of the Mössbauer effect to produce a narrow resonance line whose shift could be accurately determined. Other experiments since 1960 measured the shift of spectral lines in the Sun's gravitational field and the change in rate of atomic clocks transported aloft on aircraft, rockets and satellites. Figure 3 summarizes the important redshift experiments that have been performed since 1960 (TEGP 2.4 (c) [420]). 


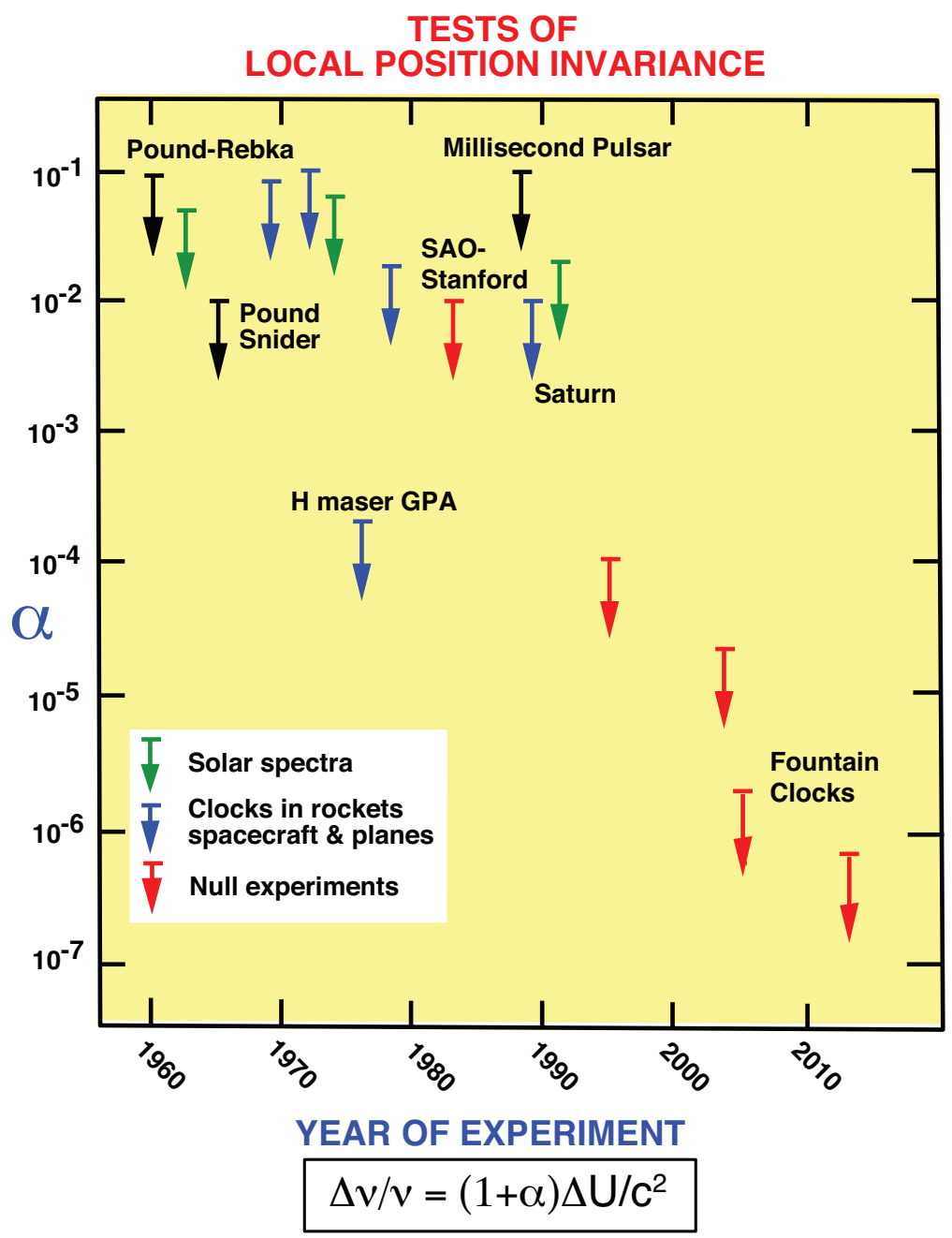

Figure 3: Selected tests of local position invariance via gravitational redshift experiments, showing bounds on $\alpha$, which measures degree of deviation of redshift from the formula $\Delta \nu / \nu=\Delta U / c^{2}$. In null redshift experiments, the bound is on the difference in $\alpha$ between different kinds of clocks.

After almost 50 years of inconclusive or contradictory measurements, the gravitational redshift of solar spectral lines was finally measured reliably. During the early years of GR, the failure to measure this effect in solar lines was seized upon by some as reason to doubt the theory (see [95] for an engaging history of this period). Unfortunately, the measurement is not simple. Solar spectral lines are subject to the "limb effect", a variation of spectral line wavelengths between the center of the solar disk and its edge or "limb"; this effect is actually a Doppler shift caused by complex convective and turbulent motions in the photosphere and lower chromosphere, and is expected to be minimized by observing at the solar limb, where the motions are predominantly transverse to the line of sight. The secret is to use strong, symmetrical lines, leading to unambiguous wavelength measurements. Successful measurements were finally made in 1962 and 1972 (TEGP 2.4 (c) [420]). In 1991, LoPresto et al. [259] measured the solar shift in agreement with LPI to about 2 percent by observing the oxygen triplet lines both in absorption in the limb and in emission just off the limb. 
The most precise standard redshift test to date was the Vessot-Levine rocket experiment known as Gravity Probe-A (GPA) that took place in June 1976 [400]. A hydrogen-maser clock was flown on a rocket to an altitude of about $10000 \mathrm{~km}$ and its frequency compared to a hydrogen-maser clock on the ground. The experiment took advantage of the masers' frequency stability by monitoring the frequency shift as a function of altitude. A sophisticated data acquisition scheme accurately eliminated all effects of the first-order Doppler shift due to the rocket's motion, while tracking data were used to determine the payload's location and the velocity (to evaluate the potential difference $\Delta U$, and the special relativistic time dilation). Analysis of the data yielded a limit $|\alpha|<2 \times 10^{-4}$.

A "null" redshift experiment performed in 1978 tested whether the relative rates of two different clocks depended upon position. Two hydrogen maser clocks and an ensemble of three superconducting-cavity stabilized oscillator (SCSO) clocks were compared over a 10-day period. During the period of the experiment, the solar potential $U / c^{2}$ within the laboratory was known to change sinusoidally with a 24 -hour period by $3 \times 10^{-13}$ because of the Earth's rotation, and to change linearly at $3 \times 10^{-12}$ per day because the Earth is 90 degrees from perihelion in April. However, analysis of the data revealed no variations of either type within experimental errors, leading to a limit on the LPI violation parameter $\left|\alpha^{\mathrm{H}}-\alpha^{\mathrm{SCSO}}\right|<2 \times 10^{-2}$ [391]. This bound has been improved using more stable frequency standards, such as atomic fountain clocks [174, 326, 34, 63]. The best current bounds, from comparing a Rubidium atomic fountain with a Cesium-133 fountain or with a hydrogen maser $[179,319]$, and from comparing transitions of two different isotopes of Dysprosium [246], hover around the one part per million mark.

The Atomic Clock Ensemble in Space (ACES) project will place both a cold trapped atom clock based on Cesium called PHARAO (Projet d'Horloge Atomique par Refroidissement d'Atomes en Orbite), and an advanced hydrogen maser clock on the International Space Station to measure the gravitational redshift to parts in $10^{6}$, as well as to carry out a number of fundamental physics experiments and to enable improvements in global timekeeping [335]. Launch is currently scheduled for May 2016.

The varying gravitational redshift of Earth-bound clocks relative to the highly stable millisecond pulsar PSR 1937+21, caused by the Earth's motion in the solar gravitational field around the Earth-Moon center of mass (amplitude $4000 \mathrm{~km}$ ), was measured to about 10 percent [383]. Two measurements of the redshift using stable oscillator clocks on spacecraft were made at the one percent level: one used the Voyager spacecraft in Saturn's gravitational field [233], while another used the Galileo spacecraft in the Sun's field [235].

The gravitational redshift could be improved to the $10^{-10}$ level using an array of laser cooled atomic clocks on board a spacecraft which would travel to within four solar radii of the Sun [270]. Sadly, the Solar Probe Plus mission, scheduled for launch in 2018, has been formulated as an exclusively heliophysics mission, and thus will not be able to test fundamental gravitational physics.

Modern advances in navigation using Earth-orbiting atomic clocks and accurate time-transfer must routinely take gravitational redshift and time-dilation effects into account. For example, the Global Positioning System (GPS) provides absolute positional accuracies of around $15 \mathrm{~m}$ (even better in its military mode), and 50 nanoseconds in time transfer accuracy, anywhere on Earth. Yet the difference in rate between satellite and ground clocks as a result of relativistic effects is a whopping 39 microseconds per day ( $46 \mu$ s from the gravitational redshift, and $-7 \mu$ s from time dilation). If these effects were not accurately accounted for, GPS would fail to function at its stated accuracy. This represents a welcome practical application of GR! (For the role of GR in GPS, see [25, 26]; for a popular essay, see [424].)

A final example of the almost "everyday" implications of the gravitational redshift is a remarkable measurement using optical clocks based on trapped aluminum ions of the frequency shift over a height of $1 / 3$ of a meter [80].

Local position invariance also refers to position in time. If LPI is satisfied, the fundamental constants of non-gravitational physics should be constants in time. Table 1 shows current bounds 
on cosmological variations in selected dimensionless constants. For discussion and references to early work, see TEGP 2.4 (c) [420] or [138]. For a comprehensive recent review both of experiments and of theoretical ideas that underlie proposals for varying constants, see [397].

Experimental bounds on varying constants come in two types: bounds on the present rate of variation, and bounds on the difference between today's value and a value in the distant past. The main example of the former type is the clock comparison test, in which highly stable atomic clocks of different fundamental type are intercompared over periods ranging from months to years (variants of the null redshift experiment). If the frequencies of the clocks depend differently on the electromagnetic fine structure constant $\alpha_{\mathrm{EM}}$, the electron-proton mass ratio $m_{\mathrm{e}} / m_{\mathrm{p}}$, or the gyromagnetic ratio of the proton $g_{\mathrm{p}}$, for example, then a limit on a drift of the fractional frequency difference translates into a limit on a drift of the constant(s). The dependence of the frequencies on the constants may be quite complex, depending on the atomic species involved. Experiments have exploited the techniques of laser cooling and trapping, and of atom fountains, in order to achieve extreme clock stability, and compared the Rubidium-87 hyperfine transition [271], the Mercury-199 ion electric quadrupole transition [49], the atomic Hydrogen $1 S-2 S$ transition [159], or an optical transition in Ytterbium-171 [318], against the ground-state hyperfine transition in Cesium-133. More recent experiments have used Strontium-87 atoms trapped in optical lattices [63] compared with Cesium to obtain $\dot{\alpha}_{\mathrm{EM}} / \alpha_{\mathrm{EM}}<6 \times 10^{-16} \mathrm{yr}^{-1}$, compared Rubidium-87 and Cesium-133 fountains [179] to obtain $\dot{\alpha}_{\mathrm{EM}} / \alpha_{\mathrm{EM}}<2.3 \times 10^{-16} \mathrm{yr}^{-1}$, or compared two isotopes of Dysprosium [246] to obtain $\dot{\alpha}_{\mathrm{EM}} / \alpha_{\mathrm{EM}}<1.3 \times 10^{-16} \mathrm{yr}^{-1}$,

The second type of bound involves measuring the relics of or signal from a process that occurred in the distant past and comparing the inferred value of the constant with the value measured in the laboratory today. One sub-type uses astronomical measurements of spectral lines at large redshift, while the other uses fossils of nuclear processes on Earth to infer values of constants early in geological history.

Earlier comparisons of spectral lines of different atoms or transitions in distant galaxies and quasars produced bounds $\alpha_{\mathrm{EM}}$ or $g_{\mathrm{p}}\left(m_{\mathrm{e}} / m_{\mathrm{p}}\right)$ on the order of a part in 10 per Hubble time [441]. Dramatic improvements in the precision of astronomical and laboratory spectroscopy, in the ability to model the complex astronomical environments where emission and absorption lines are produced, and in the ability to reach large redshift have made it possible to improve the bounds significantly. In fact, in 1999, Webb et al. [406, 296] announced that measurements of absorption lines in $\mathrm{Mg}$, $\mathrm{Al}, \mathrm{Si}, \mathrm{Cr}, \mathrm{Fe}, \mathrm{Ni}$, and $\mathrm{Zn}$ in quasars in the redshift range $0.5<Z<3.5$ indicated a smaller value of $\alpha_{\mathrm{EM}}$ in earlier epochs, namely $\Delta \alpha_{\mathrm{EM}} / \alpha_{\mathrm{EM}}=(-0.72 \pm 0.18) \times 10^{-5}$, corresponding to $\dot{\alpha}_{\mathrm{EM}} / \alpha_{\mathrm{EM}}=(6.4 \pm 1.4) \times 10^{-16} \mathrm{yr}^{-1}$ (assuming a linear drift with time). The Webb group continues to report changes in $\alpha$ over large redshifts [217]. Measurements by other groups have so far failed to confirm this non-zero effect [373, 76, 329]; an analysis of $\mathrm{Mg}$ absorption systems in quasars at $0.4<Z<2.3$ gave $\dot{\alpha}_{\mathrm{EM}} / \alpha_{\mathrm{EM}}=(-0.6 \pm 0.6) \times 10^{-16} \mathrm{yr}^{-1}$ [373]. Recent studies have also yielded no evidence for a variation in $\alpha_{\mathrm{EM}}[210,248]$

Another important set of bounds arises from studies of the "Oklo" phenomenon, a group of natural, sustained ${ }^{235} \mathrm{U}$ fission reactors that occurred in the Oklo region of Gabon, Africa, around 1.8 billion years ago. Measurements of ore samples yielded an abnormally low value for the ratio of two isotopes of Samarium, ${ }^{149} \mathrm{Sm} /{ }^{147} \mathrm{Sm}$. Neither of these isotopes is a fission product, but ${ }^{149} \mathrm{Sm}$ can be depleted by a flux of neutrons. Estimates of the neutron fluence (integrated dose) during the reactors' "on" phase, combined with the measured abundance anomaly, yield a value for the neutron cross-section for ${ }^{149} \mathrm{Sm} 1.8$ billion years ago that agrees with the modern value. However, the capture cross-section is extremely sensitive to the energy of a low-lying level $(E \sim 0.1 \mathrm{eV})$, so that a variation in the energy of this level of only $20 \mathrm{meV}$ over a billion years would change the capture cross-section from its present value by more than the observed amount. This was first analyzed in 1976 by Shlyakter [365]. Recent reanalyses of the Oklo data [101, 166, 320] lead to a bound on $\dot{\alpha}_{\mathrm{EM}}$ at the level of around $5 \times 10^{-17} \mathrm{yr}^{-1}$. 
Table 1: Bounds on cosmological variation of fundamental constants of non-gravitational physics. For an in-depth review, see [397].

\begin{tabular}{|c|c|c|c|}
\hline Constant $k$ & $\begin{array}{l}\text { Limit on } \dot{k} / k \\
\qquad\left(\mathrm{yr}^{-1}\right)\end{array}$ & Redshift & Method \\
\hline \multirow[t]{5}{*}{$\begin{array}{l}\text { Fine structure constant } \\
\left(\alpha_{\mathrm{EM}}=e^{2} / \hbar c\right)\end{array}$} & $<1.3 \times 10^{-16}$ & 0 & $\begin{array}{l}\text { Clock comparisons } \\
{[63,179,246]}\end{array}$ \\
\hline & $<0.5 \times 10^{-16}$ & 0.15 & $\begin{array}{l}\text { Oklo Natural Reactor } \\
{[101,166,320]}\end{array}$ \\
\hline & $<3.4 \times 10^{-16}$ & 0.45 & $\begin{array}{l}{ }^{187} \text { Re decay in meteorites } \\
{[312]}\end{array}$ \\
\hline & $(6.4 \pm 1.4) \times 10^{-16}$ & $0.2-3.7$ & $\begin{array}{l}\text { Spectra in distant quasars } \\
{[406,296,217]}\end{array}$ \\
\hline & $<1.2 \times 10^{-16}$ & $0.4-2.3$ & $\begin{array}{l}\text { Spectra in distant quasars } \\
{[373,76,329,210,248]}\end{array}$ \\
\hline \multirow[t]{2}{*}{$\begin{array}{l}\text { Weak interaction constant } \\
\left(\alpha_{\mathrm{W}}=G_{\mathrm{f}} m_{\mathrm{p}}^{2} c / \hbar^{3}\right)\end{array}$} & $<1 \times 10^{-11}$ & 0.15 & $\begin{array}{l}\text { Oklo Natural Reactor } \\
{[101]}\end{array}$ \\
\hline & $<5 \times 10^{-12}$ & $10^{9}$ & $\begin{array}{l}\text { Big-Bang nucleosynthesis } \\
{[269,334]}\end{array}$ \\
\hline \multirow[t]{2}{*}{ e-p mass ratio } & $<3.3 \times 10^{-15}$ & 0 & $\begin{array}{l}\text { Clock comparisons } \\
{[63]}\end{array}$ \\
\hline & $<3 \times 10^{-15}$ & $2.6-3.0$ & $\begin{array}{l}\text { Spectra in distant quasars } \\
\text { [199] }\end{array}$ \\
\hline
\end{tabular}

In a similar manner, recent reanalyses of decay rates of ${ }^{187} \mathrm{Re}$ in ancient meteorites (4.5 billion years old) gave the bound $\dot{\alpha}_{\mathrm{EM}} / \alpha_{\mathrm{EM}}<3.4 \times 10^{-16} \mathrm{yr}^{-1}[312]$.

\subsection{Theoretical frameworks for analyzing EEP}

\subsubsection{Schiff's conjecture}

Because the three parts of the Einstein equivalence principle discussed above are so very different in their empirical consequences, it is tempting to regard them as independent theoretical principles. On the other hand, any complete and self-consistent gravitation theory must possess sufficient mathematical machinery to make predictions for the outcomes of experiments that test each principle, and because there are limits to the number of ways that gravitation can be meshed with the special relativistic laws of physics, one might not be surprised if there were theoretical connections between the three sub-principles. For instance, the same mathematical formalism that produces equations describing the free fall of a hydrogen atom must also produce equations that determine the energy levels of hydrogen in a gravitational field, and thereby the ticking rate of a hydrogen maser clock. Hence a violation of EEP in the fundamental machinery of a theory that manifests itself as a violation of WEP might also be expected to show up as a violation of local position invariance. Around 1960, Leonard Schiff conjectured that this kind of connection was a necessary feature of any self-consistent theory of gravity. More precisely, Schiff's conjecture states that any complete, self-consistent theory of gravity that embodies WEP necessarily embodies EEP. In other words, the validity of WEP alone guarantees the validity of local Lorentz and position invariance, and thereby of EEP.

If Schiff's conjecture is correct, then Eötvös experiments may be seen as the direct empirical 
foundation for EEP, hence for the interpretation of gravity as a curved-spacetime phenomenon. Of course, a rigorous proof of such a conjecture is impossible (indeed, some special counter-examples are known [311,300,91]), yet a number of powerful "plausibility" arguments can be formulated.

The most general and elegant of these arguments is based upon the assumption of energy conservation. This assumption allows one to perform very simple cyclic gedanken experiments in which the energy at the end of the cycle must equal that at the beginning of the cycle. This approach was pioneered by Dicke, Nordtvedt, and Haugan (see, e.g., [181]). A system in a quantum state $A$ decays to state $B$, emitting a quantum of frequency $\nu$. The quantum falls a height $H$ in an external gravitational field and is shifted to frequency $\nu^{\prime}$, while the system in state $B$ falls with acceleration $g_{B}$. At the bottom, state $A$ is rebuilt out of state $B$, the quantum of frequency $\nu^{\prime}$, and the kinetic energy $m_{B} g_{B} H$ that state $B$ has gained during its fall. The energy left over must be exactly enough, $m_{A} g_{A} H$, to raise state $A$ to its original location. (Here an assumption of local Lorentz invariance permits the inertial masses $m_{A}$ and $m_{B}$ to be identified with the total energies of the bodies.) If $g_{A}$ and $g_{B}$ depend on that portion of the internal energy of the states that was involved in the quantum transition from $A$ to $B$ according to

$$
g_{A}=g\left(1+\frac{\alpha E_{A}}{m_{A} c^{2}}\right), \quad g_{B}=g\left(1+\frac{\alpha E_{B}}{m_{B} c^{2}}\right), \quad E_{A}-E_{B} \equiv h \nu
$$

(violation of WEP), then by conservation of energy, there must be a corresponding violation of LPI in the frequency shift of the form (to lowest order in $h \nu / m c^{2}$ )

$$
Z=\frac{\nu^{\prime}-\nu}{\nu^{\prime}}=(1+\alpha) \frac{g H}{c^{2}}=(1+\alpha) \frac{\Delta U}{c^{2}} .
$$

Haugan generalized this approach to include violations of LLI [181] (TEGP 2.5 [420]).

\subsubsection{The $T H \epsilon \mu$ formalism}

The first successful attempt to prove Schiff's conjecture more formally was made by Lightman and Lee [252]. They developed a framework called the $T H \epsilon \mu$ formalism that encompasses all metric theories of gravity and many non-metric theories (see Box 1). It restricts attention to the behavior of charged particles (electromagnetic interactions only) in an external static spherically symmetric (SSS) gravitational field, described by a potential $U$. It characterizes the motion of the charged particles in the external potential by two arbitrary functions $T(U)$ and $H(U)$, and characterizes the response of electromagnetic fields to the external potential (gravitationally modified Maxwell equations) by two functions $\epsilon(U)$ and $\mu(U)$. The forms of $T, H, \epsilon$, and $\mu$ vary from theory to theory, but every metric theory satisfies

$$
\epsilon=\mu=\left(\frac{H}{T}\right)^{1 / 2}
$$

for all $U$. This consequence follows from the action of electrodynamics with a "minimal" or metric coupling:

$$
I=-\sum_{a} m_{0 a} \int\left(-g_{\mu \nu} v_{a}^{\mu} v_{a}^{\nu}\right)^{1 / 2} d t+\sum_{a} e_{a} \int A_{\mu}\left(x_{a}^{\nu}\right) v_{a}^{\mu} d t-\frac{1}{16 \pi} \int \sqrt{-g} g^{\mu \alpha} g^{\nu \beta} F_{\mu \nu} F_{\alpha \beta} d^{4} x
$$

where the variables are defined in Box 1 , and where $F_{\mu \nu} \equiv A_{\nu, \mu}-A_{\mu, \nu}$. By identifying $g_{00}=T$ and $g_{i j}=H \delta_{i j}$ in a SSS field, $F_{i 0}=E_{i}$ and $F_{i j}=\epsilon_{i j k} B_{k}$, one obtains Eq. (9). Conversely, every theory within this class that satisfies Eq. (9) can have its electrodynamic equations cast into "metric" form. In a given non-metric theory, the functions $T, H, \epsilon$, and $\mu$ will depend in general on the 
full gravitational environment, including the potential of the Earth, Sun, and Galaxy, as well as on cosmological boundary conditions. Which of these factors has the most influence on a given experiment will depend on the nature of the experiment.

Lightman and Lee then calculated explicitly the rate of fall of a "test" body made up of interacting charged particles, and found that the rate was independent of the internal electromagnetic structure of the body (WEP) if and only if Eq. (9) was satisfied. In other words, WEP $\Rightarrow$ EEP and Schiff's conjecture was verified, at least within the restrictions built into the formalism.

\section{Box 1. The $T H \epsilon \mu$ formalism}

\section{Coordinate system and conventions:}

$x^{0}=t$ : time coordinate associated with the static nature of the static spherically symmetric (SSS) gravitational field; $\mathbf{x}=(x, y, z)$ : isotropic quasi-Cartesian spatial coordinates; spatial vector and gradient operations as in Cartesian space.

\section{Matter and field variables:}

- $m_{0 a}$ : rest mass of particle $a$.

- $e_{a}$ : charge of particle $a$.

- $x_{a}^{\mu}(t)$ : world line of particle $a$.

- $v_{a}^{\mu}=d x_{a}^{\mu} / d t$ : coordinate velocity of particle $a$.

- $A_{\mu}=$ : electromagnetic vector potential; $\mathbf{E}=\nabla A_{0}-\partial \mathbf{A} / \partial t, \mathbf{B}=\nabla \times \mathbf{A}$.

\section{Gravitational potential:}

$$
U(\mathbf{x}) \text {. }
$$

\section{Arbitrary functions:}

$$
T(U), H(U), \epsilon(U), \mu(U) \text {; EEP is satisfied if } \epsilon=\mu=(H / T)^{1 / 2} \text { for all } U .
$$

Action:

$$
I=-\sum_{a} m_{0 a} \int\left(T-H v_{a}^{2}\right)^{1 / 2} d t+\sum_{a} e_{a} \int A_{\mu}\left(x_{a}^{\nu}\right) v_{a}^{\mu} d t+(8 \pi)^{-1} \int\left(\epsilon E^{2}-\mu^{-1} B^{2}\right) d^{4} x .
$$

\section{Non-metric parameters:}

$$
\Gamma_{0}=-c_{0}^{2} \frac{\partial}{\partial U} \ln \left[\epsilon(T / H)^{1 / 2}\right]_{0}, \quad \Lambda_{0}=-c_{0}^{2} \frac{\partial}{\partial U} \ln \left[\mu(T / H)^{1 / 2}\right]_{0}, \quad \Upsilon_{0}=1-\left(T H^{-1} \epsilon \mu\right)_{0},
$$

where $c_{0}=\left(T_{0} / H_{0}\right)^{1 / 2}$ and subscript " 0 " refers to a chosen point in space. If EEP is satisfied, $\Gamma_{0} \equiv \Lambda_{0} \equiv \Upsilon_{0} \equiv 0$.

Certain combinations of the functions $T, H, \epsilon$, and $\mu$ reflect different aspects of EEP. For instance, position or $U$-dependence of either of the combinations $\epsilon(T / H)^{1 / 2}$ and $\mu(T / H)^{1 / 2}$ signals violations of LPI, the first combination playing the role of the locally measured electric charge or fine structure constant. The "non-metric parameters" $\Gamma_{0}$ and $\Lambda_{0}$ (see Box 1) are measures of such violations of EEP. Similarly, if the parameter $\Upsilon_{0} \equiv 1-\left(T H^{-1} \epsilon \mu\right)_{0}$ is non-zero anywhere, then violations of LLI will occur. This parameter is related to the difference between the speed of light $c$, and the limiting speed of material test particles $c_{0}$, given by

$$
c=\left(\epsilon_{0} \mu_{0}\right)^{-1 / 2}, \quad c_{0}=\left(\frac{T_{0}}{H_{0}}\right)^{1 / 2}
$$


In many applications, by suitable definition of units, $c_{0}$ can be set equal to unity. If EEP is valid, $\Gamma_{0} \equiv \Lambda_{0} \equiv \Upsilon_{0}=0$ everywhere.

The rate of fall of a composite spherical test body of electromagnetically interacting particles then has the form

$$
\begin{aligned}
\mathbf{a} & =\frac{m_{\mathrm{P}}}{m} \nabla U, \\
\frac{m_{\mathrm{P}}}{m} & =1+\frac{E_{\mathrm{B}}^{\mathrm{ES}}}{M c_{0}^{2}}\left[2 \Gamma_{0}-\frac{8}{3} \Upsilon_{0}\right]+\frac{E_{\mathrm{B}}^{\mathrm{MS}}}{M c_{0}^{2}}\left[2 \Lambda_{0}-\frac{4}{3} \Upsilon_{0}\right]+\ldots,
\end{aligned}
$$

where $E_{\mathrm{B}}^{\mathrm{ES}}$ and $E_{\mathrm{B}}^{\mathrm{MS}}$ are the electrostatic and magnetostatic binding energies of the body, given by

$$
\begin{aligned}
& E_{\mathrm{B}}^{\mathrm{ES}}=-\frac{1}{4} T_{0}^{1 / 2} H_{0}^{-1} \epsilon_{0}^{-1}\left\langle\sum_{a b} \frac{e_{a} e_{b}}{r_{a b}}\right\rangle, \\
& E_{\mathrm{B}}^{\mathrm{MS}}=-\frac{1}{8} T_{0}^{1 / 2} H_{0}^{-1} \mu_{0}\left\langle\sum_{a b} \frac{e_{a} e_{b}}{r_{a b}}\left[\mathbf{v}_{a} \cdot \mathbf{v}_{b}+\left(\mathbf{v}_{a} \cdot \mathbf{n}_{a b}\right)\left(\mathbf{v}_{b} \cdot \mathbf{n}_{a b}\right)\right]\right\rangle,
\end{aligned}
$$

where $r_{a b}=\left|\mathbf{x}_{a}-\mathbf{x}_{b}\right|, \mathbf{n}_{a b}=\left(\mathbf{x}_{a}-\mathbf{x}_{b}\right) / r_{a b}$, and the angle brackets denote an expectation value of the enclosed operator for the system's internal state. Eötvös experiments place limits on the WEP-violating terms in Eq. (13), and ultimately place limits on the non-metric parameters $\left|\Gamma_{0}\right|<$ $2 \times 10^{-10}$ and $\left|\Lambda_{0}\right|<3 \times 10^{-6}$. (We set $\Upsilon_{0}=0$ because of very tight constraints on it from tests of LLI; see Figure 2, where $\delta=-\Upsilon_{0}$.) These limits are sufficiently tight to rule out a number of non-metric theories of gravity thought previously to be viable (TEGP 2.6 (f) [420]).

The TH $\epsilon \mu$ formalism also yields a gravitationally modified Dirac equation that can be used to determine the gravitational redshift experienced by a variety of atomic clocks. For the redshift parameter $\alpha$ (see Eq. (6)), the results are (TEGP 2.6 (c) [420]):

$$
\alpha= \begin{cases}-3 \Gamma_{0}+\Lambda_{0} & \text { hydrogen hyperfine transition, H-Maser clock, } \\ -\frac{1}{2}\left(3 \Gamma_{0}+\Lambda_{0}\right) & \text { electromagnetic mode in cavity, SCSO clock, } \\ -2 \Gamma_{0} & \text { phonon mode in solid, principal transition in hydrogen. }\end{cases}
$$

The redshift is the standard one $(\alpha=0)$, independently of the nature of the clock if and only if $\Gamma_{0} \equiv \Lambda_{0} \equiv 0$. Thus the Vessot-Levine rocket redshift experiment sets a limit on the parameter combination $\left|3 \Gamma_{0}-\Lambda_{0}\right|$ (see Figure 3); the null-redshift experiment comparing hydrogen-maser and SCSO clocks sets a limit on $\left|\alpha_{\mathrm{H}}-\alpha_{\mathrm{SCSO}}\right|=\frac{3}{2}\left|\Gamma_{0}-\Lambda_{0}\right|$. Alvarez and Mann $[9,10,11,12,13]$ extended the $T H \epsilon \mu$ formalism to permit analysis of such effects as the Lamb shift, anomalous magnetic moments and non-baryonic effects, and placed interesting bounds on EEP violations.

\subsubsection{The $c^{2}$ formalism}

The TH $\epsilon \mu$ formalism can also be applied to tests of local Lorentz invariance, but in this context it can be simplified. Since most such tests do not concern themselves with the spatial variation of the functions $T, H, \epsilon$, and $\mu$, but rather with observations made in moving frames, we can treat them as spatial constants. Then by rescaling the time and space coordinates, the charges and the electromagnetic fields, we can put the action in Box 1 into the form (TEGP 2.6 (a) [420])

$$
I=-\sum_{a} m_{0 a} \int\left(1-v_{a}^{2}\right)^{1 / 2} d t+\sum_{a} e_{a} \int A_{\mu}\left(x_{a}^{\nu}\right) v_{a}^{\mu} d t+(8 \pi)^{-1} \int\left(E^{2}-c^{2} B^{2}\right) d^{4} x
$$


where $c^{2} \equiv H_{0} /\left(T_{0} \epsilon_{0} \mu_{0}\right)=\left(1-\Upsilon_{0}\right)^{-1}$. This amounts to using units in which the limiting speed $c_{0}$ of massive test particles is unity, and the speed of light is $c$. If $c \neq 1$, LLI is violated; furthermore, the form of the action above must be assumed to be valid only in some preferred universal rest frame. The natural candidate for such a frame is the rest frame of the microwave background.

The electrodynamical equations which follow from Eq. (17) yield the behavior of rods and clocks, just as in the full $T H \epsilon \mu$ formalism. For example, the length of a rod which moves with velocity $\mathbf{V}$ relative to the rest frame in a direction parallel to its length will be observed by a rest observer to be contracted relative to an identical rod perpendicular to the motion by a factor $1-V^{2} / 2+\mathcal{O}\left(V^{4}\right)$. Notice that $c$ does not appear in this expression, because only electrostatic interactions are involved, and $c$ appears only in the magnetic sector of the action (17). The energy and momentum of an electromagnetically bound body moving with velocity $\mathbf{V}$ relative to the rest frame are given by

$$
\begin{aligned}
E & =M_{\mathrm{R}}+\frac{1}{2} M_{\mathrm{R}} V^{2}+\frac{1}{2} \delta M_{\mathrm{I}}^{i j} V^{i} V^{j}+\mathcal{O}\left(M V^{4}\right), \\
P^{i} & =M_{\mathrm{R}} V^{i}+\delta M_{\mathrm{I}}^{i j} V^{j}+\mathcal{O}\left(M V^{3}\right),
\end{aligned}
$$

where $M_{\mathrm{R}}=M_{0}-E_{\mathrm{B}}^{\mathrm{ES}}, M_{0}$ is the sum of the particle rest masses, $E_{\mathrm{B}}^{\mathrm{ES}}$ is the electrostatic binding energy of the system (see Eq. (14) with $T_{0}^{1 / 2} H_{0} \epsilon_{0}^{-1}=1$ ), and

$$
\delta M_{\mathrm{I}}^{i j}=-2\left(\frac{1}{c^{2}}-1\right)\left[\frac{4}{3} E_{\mathrm{B}}^{\mathrm{ES}} \delta^{i j}+\tilde{E}_{\mathrm{B}}^{\mathrm{ES} i j}\right]
$$

where

$$
\tilde{E}_{\mathrm{B}}^{\mathrm{ES} i j}=-\frac{1}{4}\left\langle\sum_{a b} \frac{e_{a} e_{b}}{r_{a b}}\left(n_{a b}^{i} n_{a b}^{j}-\frac{1}{3} \delta^{i j}\right)\right\rangle .
$$

Note that $\left(c^{-2}-1\right)$ corresponds to the parameter $\delta$ plotted in Figure 2.

The electrodynamics given by Eq. (17) can also be quantized, so that we may treat the interaction of photons with atoms via perturbation theory. The energy of a photon is $\hbar$ times its frequency $\omega$, while its momentum is $\hbar \omega / c$. Using this approach, one finds that the difference in round trip travel times of light along the two arms of the interferometer in the Michelson-Morley experiment is given by $L_{0}\left(v^{2} / c\right)\left(c^{-2}-1\right)$. The experimental null result then leads to the bound on $\left(c^{-2}-1\right)$ shown on Figure 2. Similarly the anisotropy in energy levels is clearly illustrated by the tensorial terms in Eqs. $(18,20)$; by evaluating $\tilde{E}_{\mathrm{B}}^{\mathrm{ES} i j}$ for each nucleus in the various Hughes-Drever-type experiments and comparing with the experimental limits on energy differences, one obtains the extremely tight bounds also shown on Figure 2.

The behavior of moving atomic clocks can also be analyzed in detail, and bounds on $\left(c^{-2}-1\right)$ can be placed using results from tests of time dilation and of the propagation of light. In some cases, it is advantageous to combine the $c^{2}$ framework with a "kinematical" viewpoint that treats a general class of boost transformations between moving frames. Such kinematical approaches have been discussed by Robertson, Mansouri and Sexl, and Will (see [418]).

For example, in the "JPL" experiment, in which the phases of two hydrogen masers connected by a fiberoptic link were compared as a function of the Earth's orientation, the predicted phase difference as a function of direction is, to first order in $\mathbf{V}$, the velocity of the Earth through the cosmic background,

$$
\frac{\Delta \phi}{\tilde{\phi}} \approx-\frac{4}{3}\left(1-c^{2}\right)\left(\mathbf{V} \cdot \mathbf{n}-\mathbf{V} \cdot \mathbf{n}_{0}\right),
$$

where $\tilde{\phi}=2 \pi \nu L, \nu$ is the maser frequency, $L=21 \mathrm{~km}$ is the baseline, and where $\mathbf{n}$ and $\mathbf{n}_{0}$ are unit vectors along the direction of propagation of the light at a given time and at the initial time of the experiment, respectively. The observed limit on a diurnal variation in the relative phase 
resulted in the bound $\left|c^{-2}-1\right|<3 \times 10^{-4}$. Tighter bounds were obtained from a "two-photon absorption" (TPA) experiment, and a 1960s series of "Mössbauer-rotor" experiments, which tested the isotropy of time dilation between a gamma ray emitter on the rim of a rotating disk and an absorber placed at the center [418].

\subsubsection{The standard model extension (SME)}

Kostelecký and collaborators developed a useful and elegant framework for discussing violations of Lorentz symmetry in the context of the standard model of particle physics [92, 93, 228]. Called the standard model extension $(\mathrm{SME})$, it takes the standard $\mathrm{SU}(3) \times \mathrm{SU}(2) \times \mathrm{U}(1)$ field theory of particle physics, and modifies the terms in the action by inserting a variety of tensorial quantities in the quark, lepton, Higgs, and gauge boson sectors that could explicitly violate LLI. SME extends the earlier classical $T H \epsilon \mu$ and $c^{2}$ frameworks, and the $\chi-g$ framework of $\mathrm{Ni}$ [300] to quantum field theory and particle physics. The modified terms split naturally into those that are odd under CPT (i.e., that violate CPT) and terms that are even under CPT. The result is a rich and complex framework, with many parameters to be analyzed and tested by experiment. Such details are beyond the scope of this review; for a review of SME and other frameworks, the reader is referred to the Living Review by Mattingly [273] or the review by Liberati [251]. The review of the SME by Kostelecký and Russell [229] provides "data tables" showing experimental bounds on all the various parameters of the SME.

Here we confine our attention to the electromagnetic sector, in order to link the SME with the $c^{2}$ framework discussed above. In the SME, the Lagrangian for a scalar particle $\phi$ with charge $e$ interacting with electrodynamics takes the form

$$
\mathcal{L}=\left[\eta^{\mu \nu}+\left(k_{\phi}\right)^{\mu \nu}\right]\left(D_{\mu} \phi\right)^{\dagger} D_{\nu} \phi-m^{2} \phi^{\dagger} \phi-\frac{1}{4}\left[\eta^{\mu \alpha} \eta^{\nu \beta}+\left(k_{F}\right)^{\mu \nu \alpha \beta}\right] F_{\mu \nu} F_{\alpha \beta},
$$

where $D_{\mu} \phi=\partial_{\mu} \phi+i e A_{\mu} \phi$, where $\left(k_{\phi}\right)^{\mu \nu}$ is a real symmetric trace-free tensor, and where $\left(k_{F}\right)^{\mu \nu \alpha \beta}$ is a tensor with the symmetries of the Riemann tensor, and with vanishing double trace. It has 19 independent components. There could also be a CPT-odd term in $\mathcal{L}$ of the form $\left(k_{A}\right)^{\mu} \epsilon_{\mu \nu \alpha \beta} A^{\nu} F^{\alpha \beta}$, but because of a variety of pre-existing theoretical and experimental constraints, it is generally set to zero.

The tensor $\left(k_{F}\right)^{\mu \alpha \nu \beta}$ can be decomposed into "electric", "magnetic", and "odd-parity" components, by defining

$$
\begin{aligned}
\left(\kappa_{D E}\right)^{j k} & =-2\left(k_{F}\right)^{0 j 0 k}, \\
\left(\kappa_{H B}\right)^{j k} & =\frac{1}{2} \epsilon^{j p q} \epsilon^{k r s}\left(k_{F}\right)^{p q r s}, \\
\left(\kappa_{D B}\right)^{k j} & =-\left(k_{H E}\right)^{j k}=\epsilon^{j p q}\left(k_{F}\right)^{0 k p q} .
\end{aligned}
$$

In many applications it is useful to use the further decomposition

$$
\begin{aligned}
\tilde{\kappa}_{\mathrm{tr}} & =\frac{1}{3}\left(\kappa_{D E}\right)^{j j} \\
\left(\tilde{\kappa}_{\mathrm{e}+}\right)^{j k} & =\frac{1}{2}\left(\kappa_{D E}+\kappa_{H B}\right)^{j k} \\
\left(\tilde{\kappa}_{\mathrm{e}-}\right)^{j k} & =\frac{1}{2}\left(\kappa_{D E}-\kappa_{H B}\right)^{j k}-\frac{1}{3} \delta^{j k}\left(\kappa_{D E}\right)^{i i}, \\
\left(\tilde{\kappa}_{\mathrm{O}+}\right)^{j k} & =\frac{1}{2}\left(\kappa_{D B}+\kappa_{H E}\right)^{j k} \\
\left(\tilde{\kappa}_{\mathrm{O}-}\right)^{j k} & =\frac{1}{2}\left(\kappa_{D B}-\kappa_{H E}\right)^{j k} .
\end{aligned}
$$


The first expression is a single number, the next three are symmetric trace-free matrices, and the final is an antisymmetric matrix, accounting thereby for the 19 components of the original tensor $\left(k_{F}\right)^{\mu \alpha \nu \beta}$.

In the rest frame of the universe, these tensors have some form that is established by the global nature of the solutions of the overarching theory being used. In a frame that is moving relative to the universe, the tensors will have components that depend on the velocity of the frame, and on the orientation of the frame relative to that velocity.

In the case where the theory is rotationally symmetric in the preferred frame, the tensors $\left(k_{\phi}\right)^{\mu \nu}$ and $\left(k_{F}\right)^{\mu \nu \alpha \beta}$ can be expressed in the form

$$
\begin{aligned}
\left(k_{\phi}\right)^{\mu \nu} & =\tilde{\kappa}_{\phi}\left(u^{\mu} u^{\nu}+\frac{1}{4} \eta^{\mu \nu}\right), \\
\left(k_{F}\right)^{\mu \nu \alpha \beta} & =\tilde{\kappa}_{\mathrm{tr}}\left(4 u^{[\mu} \eta^{\nu][\alpha} u^{\beta]}-\eta^{\mu[\alpha} \eta^{\beta] \nu}\right),
\end{aligned}
$$

where [ ] around indices denote antisymmetrization, and where $u^{\mu}$ is the four-velocity of an observer at rest in the preferred frame. With this assumption, all the tensorial quantities in Eq. (24) vanish in the preferred frame, and, after suitable rescalings of coordinates and fields, the action (22) can be put into the form of the $c^{2}$ framework, with

$$
c=\left(\frac{1-\frac{3}{4} \tilde{\kappa}_{\phi}}{1+\frac{1}{4} \tilde{\kappa}_{\phi}}\right)^{1 / 2}\left(\frac{1-\tilde{\kappa}_{\mathrm{tr}}}{1+\tilde{\kappa}_{\mathrm{tr}}}\right)^{1 / 2} .
$$

\subsection{EEP, particle physics, and the search for new interactions}

Thus far, we have discussed EEP as a principle that strictly divides the world into metric and nonmetric theories, and have implied that a failure of EEP might invalidate metric theories (and thus general relativity). On the other hand, there is mounting theoretical evidence to suggest that EEP is likely to be violated at some level, whether by quantum gravity effects, by effects arising from string theory, or by hitherto undetected interactions. Roughly speaking, in addition to the pure Einsteinian gravitational interaction, which respects EEP, theories such as string theory predict other interactions which do not. In string theory, for example, the existence of such EEP-violating fields is assured, but the theory is not yet mature enough to enable a robust calculation of their strength relative to gravity, or a determination of whether they are long range, like gravity, or short range, like the nuclear and weak interactions, and thus too short-range to be detectable.

In one simple example [129], one can write the Lagrangian for the low-energy limit of a stringinspired theory in the so-called "Einstein frame", in which the gravitational Lagrangian is purely general relativistic:

$$
\begin{aligned}
\tilde{\mathcal{L}}=\sqrt{-\tilde{g}}\left(\tilde{g}^{\mu \nu}\left[\frac{1}{2 \kappa} \tilde{R}_{\mu \nu}-\frac{1}{2} \tilde{G}(\varphi) \partial_{\mu} \varphi \partial_{\nu} \varphi\right]-U(\varphi) \tilde{g}^{\mu \nu} \tilde{g}^{\alpha \beta} F_{\mu \alpha} F_{\nu \beta}\right. \\
\left.+\tilde{\tilde{\psi}}\left[i \tilde{e}_{a}^{\mu} \gamma^{a}\left(\partial_{\mu}+\tilde{\Omega}_{\mu}+q A_{\mu}\right)-\tilde{M}(\varphi)\right] \tilde{\psi}\right)
\end{aligned}
$$

where $\tilde{g}_{\mu \nu}$ is the non-physical metric, $\tilde{R}_{\mu \nu}$ is the Ricci tensor derived from it, $\varphi$ is a dilaton field, and $\tilde{G}, U$ and $\tilde{M}$ are functions of $\varphi$. The Lagrangian includes that for the electromagnetic field $F_{\mu \nu}$, and that for particles, written in terms of Dirac spinors $\tilde{\psi}$. This is not a metric representation because of the coupling of $\varphi$ to matter via $\tilde{M}(\varphi)$ and $U(\varphi)$. A conformal transformation $\tilde{g}_{\mu \nu}=F(\varphi) g_{\mu \nu}$, 
$\tilde{\psi}=F(\varphi)^{-3 / 4} \psi$, puts the Lagrangian in the form ("Jordan" frame)

$$
\begin{aligned}
\mathcal{L}=\sqrt{-g} & \left(g^{\mu \nu}\left[\frac{1}{2 \kappa} F(\varphi) R_{\mu \nu}-\frac{1}{2} F(\varphi) \tilde{G}(\varphi) \partial_{\mu} \varphi \partial_{\nu} \varphi+\frac{3}{4 \kappa F(\varphi)} \partial_{\mu} F \partial_{\nu} F\right]\right. \\
& \left.-U(\varphi) g^{\mu \nu} g^{\alpha \beta} F_{\mu \alpha} F_{\nu \beta}+\bar{\psi}\left[i e_{a}^{\mu} \gamma^{a}\left(\partial_{\mu}+\Omega_{\mu}+q A_{\mu}\right)-\tilde{M}(\varphi) F^{1 / 2}\right] \psi\right) .
\end{aligned}
$$

One may choose $F(\varphi)=$ const. $/ \tilde{M}(\varphi)^{2}$ so that the particle Lagrangian takes the metric form (no explicit coupling to $\varphi$ ), but the electromagnetic Lagrangian will still couple non-metrically to $U(\varphi)$. The gravitational Lagrangian here takes the form of a scalar-tensor theory (see Section 3.3.2). But the non-metric electromagnetic term will, in general, produce violations of EEP. For examples of specific models, see [384, 117]. Another class of non-metric theories is the "varying speed of light (VSL)" set of theories; for a detailed review, see [268].

On the other hand, whether one views such effects as a violation of EEP or as effects arising from additional "matter" fields whose interactions, like those of the electromagnetic field, do not fully embody EEP, is to some degree a matter of semantics. Unlike the fields of the standard model of electromagnetic, weak and strong interactions, which couple to properties other than mass-energy and are either short range or are strongly screened, the fields inspired by string theory could be long range (if they remain massless by virtue of a symmetry, or at best, acquire a very small mass), and can couple to mass-energy, and thus can mimic gravitational fields. Still, there appears to be no way to make this precise.

As a result, EEP and related tests are now viewed as ways to discover or place constraints on new physical interactions, or as a branch of "non-accelerator particle physics", searching for the possible imprints of high-energy particle effects in the low-energy realm of gravity. Whether current or proposed experiments can actually probe these phenomena meaningfully is an open question at the moment, largely because of a dearth of firm theoretical predictions.

\subsubsection{The "fifth" force}

On the phenomenological side, the idea of using EEP tests in this way may have originated in the middle 1980s, with the search for a "fifth" force. In 1986, as a result of a detailed reanalysis of Eötvös' original data, Fischbach et al. [156] suggested the existence of a fifth force of nature, with a strength of about a percent that of gravity, but with a range (as defined by the range $\lambda$ of a Yukawa potential, $e^{-r / \lambda} / r$ ) of a few hundred meters. This proposal dovetailed with earlier hints of a deviation from the inverse-square law of Newtonian gravitation derived from measurements of the gravity profile down deep mines in Australia, and with emerging ideas from particle physics suggesting the possible presence of very low-mass particles with gravitational-strength couplings. During the next four years numerous experiments looked for evidence of the fifth force by searching for composition-dependent differences in acceleration, with variants of the Eötvös experiment or with free-fall Galileo-type experiments. Although two early experiments reported positive evidence, the others all yielded null results. Over the range between one and $10^{4}$ meters, the null experiments produced upper limits on the strength of a postulated fifth force between $10^{-3}$ and $10^{-6}$ of the strength of gravity. Interpreted as tests of WEP (corresponding to the limit of infinite-range forces), the results of two representative experiments from this period, the free-fall Galileo experiment and the early Eöt-Wash experiment, are shown in Figure 1. At the same time, tests of the inverse-square law of gravity were carried out by comparing variations in gravity measurements up tall towers or down mines or boreholes with gravity variations predicted using the inverse square law together with Earth models and surface gravity data mathematically "continued" up the tower or down the hole. Despite early reports of anomalies, independent tower, borehole, and seawater measurements ultimately showed no evidence of a deviation. Analyses of orbital data from planetary range measurements, lunar laser ranging (LLR), and laser tracking of the LAGEOS satellite verified 
the inverse-square law to parts in $10^{8}$ over scales of $10^{3}$ to $10^{5} \mathrm{~km}$, and to parts in $10^{9}$ over planetary scales of several astronomical units [381]. A consensus emerged that there was no credible experimental evidence for a fifth force of nature, of a type and range proposed by Fischbach et al. For reviews and bibliographies of this episode, see [155, 157, 158, 4, 417].

\subsubsection{Short-range modifications of Newtonian gravity}

Although the idea of an intermediate-range violation of Newton's gravitational law was dropped, new ideas emerged to suggest the possibility that the inverse-square law could be violated at very short ranges, below the centimeter range of existing laboratory verifications of the $1 / r^{2}$ behavior. One set of ideas $[18,21,331,330]$ posited that some of the extra spatial dimensions that come with string theory could extend over macroscopic scales, rather than being rolled up at the Planck scale of $10^{-33} \mathrm{~cm}$, which was then the conventional viewpoint. On laboratory distances large compared to the relevant scale of the extra dimension, gravity would fall off as the inverse square, whereas on short scales, gravity would fall off as $1 / R^{2+n}$, where $n$ is the number of large extra dimensions. Many models favored $n=1$ or $n=2$. Other possibilities for effective modifications of gravity at short range involved the exchange of light scalar particles.

Following these proposals, many of the high-precision, low-noise methods that were developed for tests of WEP were adapted to carry out laboratory tests of the inverse square law of Newtonian gravitation at millimeter scales and below. The challenge of these experiments has been to distinguish gravitation-like interactions from electromagnetic and quantum mechanical (Casimir) effects. No deviations from the inverse square law have been found to date at distances between tens of nanometers and $10 \mathrm{~mm}[258,193,192,79,257,211,2,390,172,380,45,449,218]$. For a comprehensive review of both the theory and the experiments circa 2002, see [3].

\subsubsection{The Pioneer anomaly}

In 1998, Anderson et al. [16] reported the presence of an anomalous deceleration in the motion of the Pioneer 10 and 11 spacecraft at distances between 20 and 70 astronomical units from the Sun. Although the anomaly was the result of a rigorous analysis of Doppler data taken over many years, it might have been dismissed as having no real significance for new physics, where it not for the fact that the acceleration, of order $10^{-9} \mathrm{~m} / \mathrm{s}^{2}$, when divided by the speed of light, was strangely close to the inverse of the Hubble time. The Pioneer anomaly prompted an outpouring of hundreds of papers, most attempting to explain it via modifications of gravity or via new physical interactions, with a small subset trying to explain it by conventional means.

Soon after the publication of the initial Pioneer anomaly paper [16], Katz pointed out that the anomaly could be accounted for as the result of the anisotropic emission of radiation from the radioactive thermal generators (RTG) that continued to power the spacecraft decades after their launch [212]. At the time, there was insufficient data on the performance of the RTG over time or on the thermal characteristics of the spacecraft to justify more than an order-of-magnitude estimate. However, the recovery of an extended set of Doppler data covering a longer stretch of the orbits of both spacecraft, together with the fortuitous discovery of project documentation and of telemetry data giving on-board temperature information, made it possible both to improve the orbit analysis and to develop detailed thermal models of the spacecraft in order to quantify the effect of thermal emission anisotropies. Several independent analyses now confirm that the anomaly is almost entirely due to the recoil of the spacecraft from the anisotropic emission of residual thermal radiation [339, 396, 291]. For a thorough review of the Pioneer anomaly published just as the new analyses were underway, see the Living Review by Turyshev and Toth [395]. 


\section{Metric Theories of Gravity and the PPN Formalism}

\subsection{Metric theories of gravity and the strong equivalence principle}

\subsubsection{Universal coupling and the metric postulates}

The empirical evidence supporting the Einstein equivalence principle, discussed in Section 2, supports the conclusion that the only theories of gravity that have a hope of being viable are metric theories, or possibly theories that are metric apart from very weak or short-range non-metric couplings (as in string theory). Therefore for the remainder of this review, we shall turn our attention exclusively to metric theories of gravity, which assume that

1. there exists a symmetric metric,

2. test bodies follow geodesics of the metric, and

3. in local Lorentz frames, the non-gravitational laws of physics are those of special relativity.

The property that all non-gravitational fields should couple in the same manner to a single gravitational field is sometimes called "universal coupling". Because of it, one can discuss the metric as a property of spacetime itself rather than as a field over spacetime. This is because its properties may be measured and studied using a variety of different experimental devices, composed of different non-gravitational fields and particles, and, because of universal coupling, the results will be independent of the device. Thus, for instance, the proper time between two events is a characteristic of spacetime and of the location of the events, not of the clocks used to measure it.

Consequently, if EEP is valid, the non-gravitational laws of physics may be formulated by taking their special relativistic forms in terms of the Minkowski metric $\boldsymbol{\eta}$ and simply "going over" to new forms in terms of the curved spacetime metric $\boldsymbol{g}$, using the mathematics of differential geometry. The details of this "going over" can be found in standard textbooks (see [289, 407, 355, 324], TEGP 3.2. [420]).

\subsubsection{The strong equivalence principle}

In any metric theory of gravity, matter and non-gravitational fields respond only to the spacetime metric $\boldsymbol{g}$. In principle, however, there could exist other gravitational fields besides the metric, such as scalar fields, vector fields, and so on. If, by our strict definition of metric theory, matter does not couple to these fields, what can their role in gravitation theory be? Their role must be that of mediating the manner in which matter and non-gravitational fields generate gravitational fields and produce the metric; once determined, however, the metric alone acts back on the matter in the manner prescribed by EEP.

What distinguishes one metric theory from another, therefore, is the number and kind of gravitational fields it contains in addition to the metric, and the equations that determine the structure and evolution of these fields. From this viewpoint, one can divide all metric theories of gravity into two fundamental classes: "purely dynamical" and "prior-geometric".

By "purely dynamical metric theory" we mean any metric theory whose gravitational fields have their structure and evolution determined by coupled partial differential field equations. In other words, the behavior of each field is influenced to some extent by a coupling to at least one of the other fields in the theory. By "prior geometric" theory, we mean any metric theory that contains "absolute elements", fields or equations whose structure and evolution are given a priori, and are independent of the structure and evolution of the other fields of the theory. These "absolute elements" typically include flat background metrics $\boldsymbol{\eta}$ or cosmic time coordinates $t$.

General relativity is a purely dynamical theory since it contains only one gravitational field, the metric itself, and its structure and evolution are governed by partial differential equations 
(Einstein's equations). Brans-Dicke theory and its generalizations are purely dynamical theories; the field equation for the metric involves the scalar field (as well as the matter as source), and that for the scalar field involves the metric. Visser's bimetric massive gravity theory [401] is a prior-geometric theory: It has a non-dynamical, Riemann-flat background metric $\boldsymbol{\eta}$, and the field equations for the physical metric $\boldsymbol{g}$ involve $\boldsymbol{\eta}$.

By discussing metric theories of gravity from this broad point of view, it is possible to draw some general conclusions about the nature of gravity in different metric theories, conclusions that are reminiscent of the Einstein equivalence principle, but that are subsumed under the name "strong equivalence principle".

Consider a local, freely falling frame in any metric theory of gravity. Let this frame be small enough that inhomogeneities in the external gravitational fields can be neglected throughout its volume. On the other hand, let the frame be large enough to encompass a system of gravitating matter and its associated gravitational fields. The system could be a star, a black hole, the solar system, or a Cavendish experiment. Call this frame a "quasi-local Lorentz frame". To determine the behavior of the system we must calculate the metric. The computation proceeds in two stages. First we determine the external behavior of the metric and gravitational fields, thereby establishing boundary values for the fields generated by the local system, at a boundary of the quasi-local frame "far" from the local system. Second, we solve for the fields generated by the local system. But because the metric is coupled directly or indirectly to the other fields of the theory, its structure and evolution will be influenced by those fields, and in particular by the boundary values taken on by those fields far from the local system. This will be true even if we work in a coordinate system in which the asymptotic form of $g_{\mu \nu}$ in the boundary region between the local system and the external world is that of the Minkowski metric. Thus the gravitational environment in which the local gravitating system resides can influence the metric generated by the local system via the boundary values of the auxiliary fields. Consequently, the results of local gravitational experiments may depend on the location and velocity of the frame relative to the external environment. Of course, local non-gravitational experiments are unaffected since the gravitational fields they generate are assumed to be negligible, and since those experiments couple only to the metric, whose form can always be made locally Minkowskian at a given spacetime event. Local gravitational experiments might include Cavendish experiments, measurement of the acceleration of massive self-gravitating bodies, studies of the structure of stars and planets, or analyses of the periods of "gravitational clocks". We can now make several statements about different kinds of metric theories.

- A theory which contains only the metric $\boldsymbol{g}$ yields local gravitational physics which is independent of the location and velocity of the local system. This follows from the fact that the only field coupling the local system to the environment is $\boldsymbol{g}$, and it is always possible to find a coordinate system in which $\boldsymbol{g}$ takes the Minkowski form at the boundary between the local system and the external environment (neglecting inhomogeneities in the external gravitational field). Thus the asymptotic values of $g_{\mu \nu}$ are constants independent of location, and are asymptotically Lorentz invariant, thus independent of velocity. GR is an example of such a theory.

- A theory which contains the metric $\boldsymbol{g}$ and dynamical scalar fields $\varphi_{A}$ yields local gravitational physics which may depend on the location of the frame but which is independent of the velocity of the frame. This follows from the asymptotic Lorentz invariance of the Minkowski metric and of the scalar fields, but now the asymptotic values of the scalar fields may depend on the location of the frame. An example is Brans-Dicke theory, where the asymptotic scalar field determines the effective value of the gravitational constant, which can thus vary as $\varphi$ varies. On the other hand, a form of velocity dependence in local physics can enter indirectly if the asymptotic values of the scalar field vary with time cosmologically. Then the rate of variation of the gravitational constant could depend on the velocity of the frame. 
- A theory which contains the metric $\boldsymbol{g}$ and additional dynamical vector or tensor fields or prior-geometric fields yields local gravitational physics which may have both location and velocity-dependent effects. An example is the Einstein-Æther theory, which contains a dynamical timelike four-vector field; the large-scale distribution of matter establishes a frame in which the vector has no spatial components, and systems moving relative to that frame can experience motion-dependent effects.

These ideas can be summarized in the strong equivalence principle (SEP), which states that:

1. WEP is valid for self-gravitating bodies as well as for test bodies.

2. The outcome of any local test experiment is independent of the velocity of the (freely falling) apparatus.

3. The outcome of any local test experiment is independent of where and when in the universe it is performed.

The distinction between SEP and EEP is the inclusion of bodies with self-gravitational interactions (planets, stars) and of experiments involving gravitational forces (Cavendish experiments, gravimeter measurements). Note that SEP contains EEP as the special case in which local gravitational forces are ignored. For further discussion of SEP and EEP, see [128].

The above discussion of the coupling of auxiliary fields to local gravitating systems indicates that if SEP is strictly valid, there must be one and only one gravitational field in the universe, the metric $\boldsymbol{g}$. These arguments are only suggestive however, and no rigorous proof of this statement is available at present. Empirically it has been found that almost every metric theory other than GR introduces auxiliary gravitational fields, either dynamical or prior geometric, and thus predicts violations of SEP at some level (here we ignore quantum-theory inspired modifications to GR involving " $R^{2}$ " terms). The one exception is Nordström's 1913 conformally-flat scalar theory [303], which can be written purely in terms of the metric; the theory satisfies SEP, but unfortunately violates experiment by predicting no deflection of light. General relativity seems to be the only viable metric theory that embodies SEP completely. In Section 4.3, we shall discuss experimental evidence for the validity of SEP.

\subsection{The parametrized post-Newtonian formalism}

Despite the possible existence of long-range gravitational fields in addition to the metric in various metric theories of gravity, the postulates of those theories demand that matter and nongravitational fields be completely oblivious to them. The only gravitational field that enters the equations of motion is the metric $\boldsymbol{g}$. The role of the other fields that a theory may contain can only be that of helping to generate the spacetime curvature associated with the metric. Matter may create these fields, and they plus the matter may generate the metric, but they cannot act back directly on the matter. Matter responds only to the metric.

Thus the metric and the equations of motion for matter become the primary entities for calculating observable effects, and all that distinguishes one metric theory from another is the particular way in which matter and possibly other gravitational fields generate the metric.

The comparison of metric theories of gravity with each other and with experiment becomes particularly simple when one takes the slow-motion, weak-field limit. This approximation, known as the post-Newtonian limit, is sufficiently accurate to encompass most solar-system tests that can be performed in the foreseeable future. It turns out that, in this limit, the spacetime metric $\boldsymbol{g}$ predicted by nearly every metric theory of gravity has the same structure. It can be written as an expansion about the Minkowski metric $\left(\eta_{\mu \nu}=\operatorname{diag}(-1,1,1,1)\right)$ in terms of dimensionless 
gravitational potentials of varying degrees of smallness. These potentials are constructed from the matter variables (see Box 2) in imitation of the Newtonian gravitational potential

$$
U(\mathbf{x}, t) \equiv \int \rho\left(\mathbf{x}^{\prime}, t\right)\left|\mathbf{x}-\mathbf{x}^{\prime}\right|^{-1} d^{3} x^{\prime} .
$$

The "order of smallness" is determined according to the rules $U \sim v^{2} \sim \Pi \sim p / \rho \sim \epsilon, v^{i} \sim$ $|d / d t| /|d / d x| \sim \epsilon^{1 / 2}$, and so on (we use units in which $G=c=1$; see Box 2 for definitions and conventions).

A consistent post-Newtonian limit requires determination of $g_{00}$ correct through $\mathcal{O}\left(\epsilon^{2}\right), g_{0 i}$ through $\mathcal{O}\left(\epsilon^{3 / 2}\right)$, and $g_{i j}$ through $\mathcal{O}(\epsilon)$ (for details see TEGP 4.1 [420]). The only way that one metric theory differs from another is in the numerical values of the coefficients that appear in front of the metric potentials. The parametrized post-Newtonian (PPN) formalism inserts parameters in place of these coefficients, parameters whose values depend on the theory under study. In the current version of the PPN formalism, summarized in Box 2, ten parameters are used, chosen in such a manner that they measure or indicate general properties of metric theories of gravity (see Table 2). Under reasonable assumptions about the kinds of potentials that can be present at post-Newtonian order (basically only Poisson-like potentials of conventional perfect fluid sources, absence of parity-violating potentials), one finds that ten PPN parameters exhaust the possibilities.

Table 2: The PPN Parameters and their significance (note that $\alpha_{3}$ has been shown twice to indicate that it is a measure of two effects).

\begin{tabular}{llccc}
\hline Parameter & \multicolumn{1}{c}{$\begin{array}{c}\text { What it measures relative } \\
\text { to GR }\end{array}$} & $\begin{array}{c}\text { Value in } \\
\text { GR }\end{array}$ & $\begin{array}{c}\text { Value in semi- } \\
\text { conservative } \\
\text { theories }\end{array}$ & $\begin{array}{c}\text { Value in fully } \\
\text { conservative } \\
\text { theories }\end{array}$ \\
\hline$\gamma$ & $\begin{array}{l}\text { How much space-curvature } \\
\text { produced by unit rest mass? }\end{array}$ & 1 & $\gamma$ & $\gamma$ \\
\hline$\beta$ & How much "nonlinearity" in & 1 & $\beta$ & $\beta$ \\
& the superposition law for & & & \\
\hline$\xi$ & gravity? & 0 & $\xi$ & $\xi$ \\
\hline$\alpha_{1}$ & Preferred-location effects? & 0 & $\alpha_{1}$ & 0 \\
$\alpha_{2}$ & Preferred-frame effects? & 0 & $\alpha_{2}$ & 0 \\
$\alpha_{3}$ & & 0 & 0 & 0 \\
\hline$\alpha_{3}$ & Violation of conservation & 0 & 0 & 0 \\
$\zeta_{1}$ & of total momentum? & 0 & 0 & 0 \\
$\zeta_{2}$ & & 0 & 0 & 0 \\
$\zeta_{3}$ & & 0 & 0 & 0 \\
$\zeta_{4}$ & & 0 & 0 & 0 \\
\hline
\end{tabular}

The parameters $\gamma$ and $\beta$ are the usual Eddington-Robertson-Schiff parameters used to describe the "classical" tests of GR, and are in some sense the most important; they are the only nonzero parameters in GR and scalar-tensor gravity. The parameter $\xi$ is non-zero in any theory of gravity that predicts preferred-location effects such as a galaxy-induced anisotropy in the local gravitational constant $G_{\mathrm{L}}$ (also called "Whitehead" effects); $\alpha_{1}, \alpha_{2}, \alpha_{3}$ measure whether or not the theory predicts post-Newtonian preferred-frame effects; $\alpha_{3}, \zeta_{1}, \zeta_{2}, \zeta_{3}, \zeta_{4}$ measure whether or not the theory predicts violations of global conservation laws for total momentum. In Table 2 we show the values these parameters take 
1. in GR,

2. in any theory of gravity that possesses conservation laws for total momentum, called "semiconservative" (any theory that is based on an invariant action principle is semi-conservative), and

3. in any theory that in addition possesses six global conservation laws for angular momentum, called "fully conservative" (such theories automatically predict no post-Newtonian preferredframe effects).

Semi-conservative theories have five free PPN parameters $\left(\gamma, \beta, \xi, \alpha_{1}, \alpha_{2}\right)$ while fully conservative theories have three $(\gamma, \beta, \xi)$.

The PPN formalism was pioneered by Kenneth Nordtvedt [305], who studied the post-Newtonian metric of a system of gravitating point masses, extending earlier work by Eddington, Robertson and Schiff (TEGP 4.2 [420]). Will [413] generalized the framework to perfect fluids. A general and unified version of the PPN formalism was developed by Will and Nordtvedt [431]. The canonical version, with conventions altered to be more in accord with standard textbooks such as [289], is discussed in detail in TEGP 4 [420]. Other versions of the PPN formalism have been developed to deal with point masses with charge, fluid with anisotropic stresses, bodies with strong internal gravity, and post-post-Newtonian effects (TEGP 4.2, 14.2 [420]). Additional parameters or potentials are needed to deal with some theories, such as theories with massive fields (Yukawa-type potentials replace Poisson potentials), or theories like Chern-Simons theory, which permit parity violation in gravity.

Box 2. The Parametrized Post-Newtonian formalism

\section{Coordinate system:}

The framework uses a nearly globally Lorentz coordinate system in which the coordinates are $\left(t, x^{1}, x^{2}, x^{3}\right)$. Three-dimensional, Euclidean vector notation is used throughout. All coordinate arbitrariness ("gauge freedom") has been removed by specialization of the coordinates to the standard PPN gauge (TEGP 4.2 [420]). Units are chosen so that $G=c=1$, where $G$ is the physically measured Newtonian constant far from the solar system.

\section{Matter variables:}

- $\rho$ : density of rest mass as measured in a local freely falling frame momentarily comoving with the gravitating matter.

- $v^{i}=\left(d x^{i} / d t\right)$ : coordinate velocity of the matter.

- $w^{i}$ : coordinate velocity of the PPN coordinate system relative to the mean rest-frame of the universe.

- $p$ : pressure as measured in a local freely falling frame momentarily comoving with the matter.

- $\Pi$ : internal energy per unit rest mass (it includes all forms of non-rest-mass, non-gravitational energy, e.g., energy of compression and thermal energy).

\section{PPN parameters:}

$\gamma, \beta, \xi, \alpha_{1}, \alpha_{2}, \alpha_{3}, \zeta_{1}, \zeta_{2}, \zeta_{3}, \zeta_{4}$. 


\section{Metric:}

$$
\begin{aligned}
g_{00}= & -1+2 U-2 \beta U^{2}-2 \xi \Phi_{W}+\left(2 \gamma+2+\alpha_{3}+\zeta_{1}-2 \xi\right) \Phi_{1}+2\left(3 \gamma-2 \beta+1+\zeta_{2}+\xi\right) \Phi_{2} \\
& +2\left(1+\zeta_{3}\right) \Phi_{3}+2\left(3 \gamma+3 \zeta_{4}-2 \xi\right) \Phi_{4}-\left(\zeta_{1}-2 \xi\right) \mathcal{A}-\left(\alpha_{1}-\alpha_{2}-\alpha_{3}\right) w^{2} U-\alpha_{2} w^{i} w^{j} U_{i j} \\
& +\left(2 \alpha_{3}-\alpha_{1}\right) w^{i} V_{i}+\mathcal{O}\left(\epsilon^{3}\right), \\
g_{0 i}= & -\frac{1}{2}\left(4 \gamma+3+\alpha_{1}-\alpha_{2}+\zeta_{1}-2 \xi\right) V_{i}-\frac{1}{2}\left(1+\alpha_{2}-\zeta_{1}+2 \xi\right) W_{i}-\frac{1}{2}\left(\alpha_{1}-2 \alpha_{2}\right) w^{i} U \\
& -\alpha_{2} w^{j} U_{i j}+\mathcal{O}\left(\epsilon^{5 / 2}\right), \\
g_{i j}= & (1+2 \gamma U) \delta_{i j}+\mathcal{O}\left(\epsilon^{2}\right) .
\end{aligned}
$$

Metric potentials:

$$
\begin{aligned}
& U=\int \frac{\rho^{\prime}}{\left|\mathbf{x}-\mathbf{x}^{\prime}\right|} d^{3} x^{\prime}, \\
& U_{i j}=\int \frac{\rho^{\prime}\left(x-x^{\prime}\right)_{i}\left(x-x^{\prime}\right)_{j}}{\left|\mathbf{x}-\mathbf{x}^{\prime}\right|^{3}} d^{3} x^{\prime}, \\
& \Phi_{W}=\int \frac{\rho^{\prime} \rho^{\prime \prime}\left(\mathbf{x}-\mathbf{x}^{\prime}\right)}{\left|\mathbf{x}-\mathbf{x}^{\prime}\right|^{3}} \cdot\left(\frac{\mathbf{x}^{\prime}-\mathbf{x}^{\prime \prime}}{\left|\mathbf{x}-\mathbf{x}^{\prime \prime}\right|}-\frac{\mathbf{x}-\mathbf{x}^{\prime \prime}}{\left|\mathbf{x}^{\prime}-\mathbf{x}^{\prime \prime}\right|}\right) d^{3} x^{\prime} d^{3} x^{\prime \prime}, \\
& \mathcal{A}=\int \frac{\rho^{\prime}\left[\mathbf{v}^{\prime} \cdot\left(\mathbf{x}-\mathbf{x}^{\prime}\right)\right]^{2}}{\left|\mathbf{x}-\mathbf{x}^{\prime}\right|^{3}} d^{3} x^{\prime}, \\
& \Phi_{1}=\int \frac{\rho^{\prime} v^{\prime 2}}{\left|\mathbf{x}-\mathbf{x}^{\prime}\right|} d^{3} x^{\prime} \\
& \Phi_{2}=\int \frac{\rho^{\prime} U^{\prime}}{\left|\mathbf{x}-\mathbf{x}^{\prime}\right|} d^{3} x^{\prime}, \\
& \Phi_{3}=\int \frac{\rho^{\prime} \Pi^{\prime}}{\left|\mathbf{x}-\mathbf{x}^{\prime}\right|} d^{3} x^{\prime}, \\
& \Phi_{4}=\int \frac{p^{\prime}}{\left|\mathbf{x}-\mathbf{x}^{\prime}\right|} d^{3} x^{\prime} \\
& V_{i}=\int \frac{\rho^{\prime} v_{i}^{\prime}}{\left|\mathbf{x}-\mathbf{x}^{\prime}\right|} d^{3} x^{\prime} \\
& W_{i}=\int \frac{\rho^{\prime}\left[\mathbf{v}^{\prime} \cdot\left(\mathbf{x}-\mathbf{x}^{\prime}\right)\right]\left(x-x^{\prime}\right)_{i}}{\left|\mathbf{x}-\mathbf{x}^{\prime}\right|^{3}} d^{3} x^{\prime} .
\end{aligned}
$$

Stress-energy tensor (perfect fluid):

$$
\begin{aligned}
T^{00} & =\rho\left(1+\Pi+v^{2}+2 U\right), \\
T^{0 i} & =\rho v^{i}\left(1+\Pi+v^{2}+2 U+\frac{p}{\rho}\right), \\
T^{i j} & =\rho v^{i} v^{j}\left(1+\Pi+v^{2}+2 U+\frac{p}{\rho}\right)+p \delta^{i j}(1-2 \gamma U) .
\end{aligned}
$$

\section{Equations of motion:}

- Stressed matter: $T_{; \nu}^{\mu \nu}=0$.

- Test bodies: $\frac{d^{2} x^{\mu}}{d \lambda^{2}}+\Gamma_{\nu \lambda}^{\mu} \frac{d x^{\nu}}{d \lambda} \frac{d x^{\lambda}}{d \lambda}=0$.

- Maxwell's equations: $F_{; \nu}^{\mu \nu}=4 \pi J^{\mu}, \quad F_{\mu \nu}=A_{\nu ; \mu}-A_{\mu ; \nu}$. 


\subsection{Competing theories of gravity}

One of the important applications of the PPN formalism is the comparison and classification of alternative metric theories of gravity. The population of viable theories has fluctuated over the years as new effects and tests have been discovered, largely through the use of the PPN framework, which eliminated many theories thought previously to be viable. The theory population has also fluctuated as new, potentially viable theories have been invented.

In this review, we shall focus on GR, the general class of scalar-tensor modifications of it, of which the Jordan-Fierz-Brans-Dicke theory (Brans-Dicke, for short) is the classic example, and vector-tensor theories. The reasons are several-fold:

- A full compendium of alternative theories circa 1981 is given in TEGP 5 [420].

- Many alternative metric theories developed during the 1970s and 1980s could be viewed as "straw-man" theories, invented to prove that such theories exist or to illustrate particular properties. Few of these could be regarded as well-motivated theories from the point of view, say, of field theory or particle physics.

- A number of theories fall into the class of "prior-geometric" theories, with absolute elements such as a flat background metric in addition to the physical metric. Most of these theories predict "preferred-frame" effects, that have been tightly constrained by observations (see Section 4.3.2). An example is Rosen's bimetric theory.

- A large number of alternative theories of gravity predict gravitational wave emission substantially different from that of GR, in strong disagreement with observations of the binary pulsar (see Section 9).

- Scalar-tensor modifications of GR have become very popular in unification schemes such as string theory, and in cosmological model building. Because the scalar fields could be massive, the potentials in the post-Newtonian limit could be modified by Yukawa-like terms.

- Theories that also incorporate vector fields have attracted recent attention, in the spirit of the SME (see Section 2.2.4), as models for violations of Lorentz invariance in the gravitational sector, and as potential candidates to account for phenomena such as galaxy rotation curves without resorting to dark matter.

\subsubsection{General relativity}

The metric $\boldsymbol{g}$ is the sole dynamical field, and the theory contains no arbitrary functions or parameters, apart from the value of the Newtonian coupling constant $G$, which is measurable in laboratory experiments. Throughout this article, we ignore the cosmological constant $\Lambda_{\mathrm{C}}$. We do this despite recent evidence, from supernova data, of an accelerating universe, which would indicate either a non-zero cosmological constant or a dynamical "dark energy" contributing about 70 percent of the critical density. Although $\Lambda_{\mathrm{C}}$ has significance for quantum field theory, quantum gravity, and cosmology, on the scale of the solar-system or of stellar systems its effects are negligible, for the values of $\Lambda_{\mathrm{C}}$ inferred from supernova observations.

The field equations of GR are derivable from an invariant action principle $\delta I=0$, where

$$
I=(16 \pi G)^{-1} \int R(-g)^{1 / 2} d^{4} x+I_{\mathrm{m}}\left(\psi_{\mathrm{m}}, g_{\mu \nu}\right),
$$

where $R$ is the Ricci scalar, and $I_{\mathrm{m}}$ is the matter action, which depends on matter fields $\psi_{\mathrm{m}}$ universally coupled to the metric $\boldsymbol{g}$. By varying the action with respect to $g_{\mu \nu}$, we obtain the field 
equations

$$
G_{\mu \nu} \equiv R_{\mu \nu}-\frac{1}{2} g_{\mu \nu} R=8 \pi G T_{\mu \nu},
$$

where $T_{\mu \nu}$ is the matter energy-momentum tensor. General covariance of the matter action implies the equations of motion $T_{; \nu}^{\mu \nu}=0$; varying $I_{\mathrm{m}}$ with respect to $\psi_{\mathrm{m}}$ yields the matter field equations of the standard model. By virtue of the absence of prior-geometric elements, the equations of motion are also a consequence of the field equations via the Bianchi identities $G_{; \nu}^{\mu \nu}=0$. According to our choice of units, we set $G=1$.

The general procedure for deriving the post-Newtonian limit of metric theories is spelled out in TEGP 5.1 [420], and is described in detail for GR in TEGP 5.2 [420] (see also Chapters 6-8 of [324]). The PPN parameter values are listed in Table 3.

Table 3: Metric theories and their PPN parameter values $\left(\alpha_{3}=\zeta_{i}=0\right.$ for all cases). The parameters $\gamma^{\prime}$, $\beta^{\prime}, \alpha_{1}^{\prime}$, and $\alpha_{2}^{\prime}$ denote complicated functions of the arbitrary constants and matching parameters.

\begin{tabular}{|c|c|c|c|c|c|c|c|}
\hline \multirow[t]{2}{*}{ Theory } & \multirow{2}{*}{$\begin{array}{c}\text { Arbitrary } \\
\text { functions } \\
\text { or constants }\end{array}$} & \multirow{2}{*}{$\begin{array}{c}\text { Cosmic } \\
\text { matching } \\
\text { parameters }\end{array}$} & \multicolumn{5}{|c|}{ PPN parameters } \\
\hline & & & $\gamma$ & $\beta$ & $\xi$ & $\alpha_{1}$ & $\alpha_{2}$ \\
\hline General relativity & none & none & 1 & 1 & 0 & 0 & 0 \\
\hline \multicolumn{8}{|l|}{ Scalar-tensor } \\
\hline Brans-Dicke & $\omega_{\mathrm{BD}}$ & $\phi_{0}$ & $\frac{1+\omega_{\mathrm{BD}}}{2+\omega_{\mathrm{BD}}}$ & 1 & 0 & 0 & 0 \\
\hline General, $f(R)$ & $A(\varphi), V(\varphi)$ & $\varphi_{0}$ & $\frac{1+\omega}{2+\omega}$ & $1+\frac{\lambda}{4+2 \omega}$ & 0 & 0 & 0 \\
\hline \multicolumn{8}{|l|}{ Vector-tensor } \\
\hline Unconstrained & $\omega, c_{1}, c_{2}, c_{3}, c_{4}$ & $u$ & $\gamma^{\prime}$ & $\beta^{\prime}$ & 0 & $\alpha_{1}^{\prime}$ & $\alpha_{2}^{\prime}$ \\
\hline Einstein-Æther & $c_{1}, c_{2}, c_{3}, c_{4}$ & none & 1 & 1 & 0 & $\alpha_{1}^{\prime}$ & $\alpha_{2}^{\prime}$ \\
\hline Khronometric & $\alpha_{k}, \beta_{k}, \lambda_{k}$ & none & 1 & 1 & 0 & $\alpha_{1}^{\prime}$ & $\alpha_{2}^{\prime}$ \\
\hline Tensor-Vector-Scalar & $k, c_{1}, c_{2}, c_{3}, c_{4}$ & $\phi_{0}$ & 1 & 1 & 0 & $\alpha_{1}^{\prime}$ & $\alpha_{2}^{\prime}$ \\
\hline
\end{tabular}

\subsubsection{Scalar-tensor theories}

These theories contain the metric $\boldsymbol{g}$, a scalar field $\varphi$, a potential function $V(\varphi)$, and a coupling function $A(\varphi)$ (generalizations to more than one scalar field have also been carried out [102]). For some purposes, the action is conveniently written in a non-metric representation, sometimes denoted the "Einstein frame", in which the gravitational action looks exactly like that of GR and the scalar action looks like a minimally coupled scalar field with a potential:

$$
\tilde{I}=(16 \pi G)^{-1} \int\left[\tilde{R}-2 \tilde{g}^{\mu \nu} \partial_{\mu} \varphi \partial_{\nu} \varphi-V(\varphi)\right](-\tilde{g})^{1 / 2} d^{4} x+I_{\mathrm{m}}\left(\psi_{\mathrm{m}}, A^{2}(\varphi) \tilde{g}_{\mu \nu}\right)
$$

where $\tilde{R} \equiv \tilde{g}^{\mu \nu} \tilde{R}_{\mu \nu}$ is the Ricci scalar of the "Einstein" metric $\tilde{g}_{\mu \nu}$. (Apart from the scalar potential term $V(\varphi)$, this corresponds to Eq. (28) with $\tilde{G}(\varphi) \equiv(4 \pi G)^{-1}, U(\varphi) \equiv 1$, and $\tilde{M}(\varphi) \propto A(\varphi)$.) This representation is a "non-metric" one because the matter fields $\psi_{\mathrm{m}}$ couple to a combination 
of $\varphi$ and $\tilde{g}_{\mu \nu}$. Despite appearances, however, it is a metric theory, because it can be put into a metric representation by identifying the "physical metric"

$$
g_{\mu \nu} \equiv A^{2}(\varphi) \tilde{g}_{\mu \nu}
$$

The action can then be rewritten in the metric form

$$
I=(16 \pi G)^{-1} \int\left[\phi R-\phi^{-1} \omega(\phi) g^{\mu \nu} \partial_{\mu} \phi \partial_{\nu} \phi-\phi^{2} V\right](-g)^{1 / 2} d^{4} x+I_{\mathrm{m}}\left(\psi_{\mathrm{m}}, g_{\mu \nu}\right),
$$

where

$$
\begin{aligned}
\phi & \equiv A(\varphi)^{-2}, \\
3+2 \omega(\phi) & \equiv \alpha(\varphi)^{-2}, \\
\alpha(\varphi) & \equiv \frac{d(\ln A(\varphi))}{d \varphi} .
\end{aligned}
$$

The Einstein frame is useful for discussing general characteristics of such theories, for numerical relativity calculations, and for some cosmological applications, while the metric representation is most useful for calculating observable effects. The field equations, post-Newtonian limit and PPN parameters are discussed in TEGP 5.3 [420] (see also Section 13.5 of [324]), and the values of the PPN parameters are listed in Table 3.

The parameters that enter the post-Newtonian limit are

$$
\omega \equiv \omega\left(\phi_{0}\right), \quad \lambda \equiv\left[\frac{\phi d \omega / d \phi}{(3+2 \omega)(4+2 \omega)}\right]_{\phi_{0}},
$$

where $\phi_{0}$ is the value of $\phi$ today far from the system being studied, as determined by appropriate cosmological boundary conditions. The Newtonian gravitational constant $G_{N}$, which is set equal to unity by our choice of units, is related to the coupling constant $G, \phi_{0}$ and $\omega$ by

$$
G_{N} \equiv 1=\frac{G}{\phi_{0}}\left(\frac{4+2 \omega}{3+2 \omega}\right)_{0}
$$

In Brans-Dicke theory $\left(\omega(\phi) \equiv \omega_{\mathrm{BD}}=\right.$ const. $)$, the larger the value of $\omega_{\mathrm{BD}}$, the smaller the effects of the scalar field, and in the limit $\omega_{\mathrm{BD}} \rightarrow \infty\left(\alpha_{0} \rightarrow 0\right)$, the theory becomes indistinguishable from GR in all its predictions. In more general theories, the function $\omega(\phi)$ could have the property that, at the present epoch, and in weak-field situations, the value of the scalar field $\phi_{0}$ is such that $\omega$ is very large and $\lambda$ is very small (theory almost identical to GR today), but that for past or future values of $\phi$, or in strong-field regions such as the interiors of neutron stars, $\omega$ and $\lambda$ could take on values that would lead to significant differences from GR. It is useful to point out that all versions of scalar-tensor gravity predict that $\gamma \leq 1$ (see Table 3 ).

Damour and Esposito-Farèse [102] have adopted an alternative parametrization of scalar-tensor theories, in which one expands $\ln A(\varphi)$ about a cosmological background field value $\varphi_{0}$ :

$$
\ln A(\varphi)=\alpha_{0}\left(\varphi-\varphi_{0}\right)+\frac{1}{2} \beta_{0}\left(\varphi-\varphi_{0}\right)^{2}+\ldots
$$

A precisely linear coupling function produces Brans-Dicke theory, with $\alpha_{0}^{2}=1 /\left(2 \omega_{\mathrm{BD}}+3\right)$, or $1 /\left(2+\omega_{\mathrm{BD}}\right)=2 \alpha_{0}^{2} /\left(1+\alpha_{0}^{2}\right)$. The function $\ln A(\varphi)$ acts as a potential for the scalar field $\varphi$ within matter, and, if $\beta_{0}>0$, then during cosmological evolution, the scalar field naturally evolves toward the minimum of the potential, i.e., toward $\alpha \approx 0, \omega \rightarrow \infty$, or toward a theory close to, though not precisely GR $[112,113]$. Estimates of the expected relic deviations from GR today in such theories depend on the cosmological model, but range from $10^{-5}$ to a few times $10^{-7}$ for $|\gamma-1|$. 
Negative values of $\beta_{0}$ correspond to a "locally unstable" scalar potential (the overall theory is still stable in the sense of having no tachyons or ghosts). In this case, objects such as neutron stars can experience a "spontaneous scalarization", whereby the interior values of $\varphi$ can be very different from the exterior values, through non-linear interactions between strong gravity and the scalar field, dramatically affecting the stars' internal structure and leading to strong violations of SEP [103, 104]. There is evidence from recent numerical simulations of the occurrence of a dynamically induced scalarization during the inspirals of compact binary systems containing neutron stars, which can affect both the final motion and the gravitational-wave emission [32, 313, 364].

On the other hand, in the case $\beta_{0}<0$, one must confront that fact that, with an unstable $\varphi$ potential, cosmological evolution would presumably drive the system away from the peak where $\alpha \approx 0$, toward parameter values that could be excluded by solar system experiments.

Scalar fields coupled to gravity or matter are also ubiquitous in particle-physics-inspired models of unification, such as string theory [384, 266, 117, 114, 115]. In some models, the coupling to matter may lead to violations of EEP, which could be tested or bounded by the experiments described in Section 2.1. In many models the scalar field could be massive; if the Compton wavelength is of macroscopic scale, its effects are those of a "fifth force". Only if the theory can be cast as a metric theory with a scalar field of infinite range or of range long compared to the scale of the system in question (solar system) can the PPN framework be strictly applied. If the mass of the scalar field is sufficiently large that its range is microscopic, then, on solar-system scales, the scalar field is suppressed, and the theory is essentially equivalent to general relativity.

For a detailed review of scalar-tensor theories see [167].

\subsection{3 $f(R)$ theories}

These are theories whose action has the form

$$
I=\frac{c^{3}}{16 \pi G} \int f(R)(-g)^{1 / 2} d^{4} x+I_{\mathrm{m}}\left(\psi_{\mathrm{m}}, g_{\mu \nu}\right),
$$

where $f$ is a function chosen so that at cosmological scales, the universe will experience accelerated expansion without resorting to either a cosmological constant or dark energy. However, it turns out that such theories are equivalent to scalar-tensor theories: replace $f(R)$ by $f(\chi)-f, \chi(\chi)(R-\chi)$, where $\chi$ is a dynamical scalar field. Varying the action with respect to $\chi$ yields $f_{, \chi \chi}(R-\chi)=0$, which implies that $\chi=R$ as long as $f_{, \chi \chi} \neq 0$. Then defining a scalar field $\phi \equiv f_{, \chi}(\chi)$ one puts the action into the form of a scalar-tensor theory given by Eq. (35), with $\omega(\phi)=0$ and $\phi^{2} V=\phi \chi(\phi)-f(\chi(\phi))$. As we will see, this value of $\omega$ would ordinarily strongly violate solarsystem experiments, but it turns out that in many models, the potential $V(\phi)$ has the effect of giving the scalar field a large effective mass in the presence of matter (the so-called "chameleon mechanism" [216]), so that the scalar field is suppressed at distances that extend outside bodies like the Sun and Earth. In this way, with only modest fine tuning, $f(R)$ theories can claim to obey standard tests, while providing interesting, non general-relativistic behavior on cosmic scales. For detailed reviews of this class of theories, see [370] and [122].

\subsubsection{Vector-tensor theories}

These theories contain the metric $\boldsymbol{g}$ and a dynamical, typically timelike, four-vector field $u^{\mu}$. In some models, the four-vector is unconstrained, while in others, called Einstein-Æther theories it is constrained to be timelike with unit norm. The most general action for such theories that is quadratic in derivatives of the vector is given by

$$
I=(16 \pi G)^{-1} \int\left[\left(1+\omega u_{\mu} u^{\mu}\right) R-K_{\alpha \beta}^{\mu \nu} \nabla_{\mu} u^{\alpha} \nabla_{\nu} u^{\beta}+\lambda\left(u_{\mu} u^{\mu}+1\right)\right](-g)^{1 / 2} d^{4} x+I_{\mathrm{m}}\left(\psi_{\mathrm{m}}, g_{\mu \nu}\right),
$$


where

$$
K_{\alpha \beta}^{\mu \nu}=c_{1} g^{\mu \nu} g_{\alpha \beta}+c_{2} \delta_{\alpha}^{\mu} \delta_{\beta}^{\nu}+c_{3} \delta_{\beta}^{\mu} \delta_{\alpha}^{\nu}-c_{4} u^{\mu} u^{\nu} g_{\alpha \beta} .
$$

The coefficients $c_{i}$ are arbitrary. In the unconstrained theories, $\lambda \equiv 0$ and $\omega$ is arbitrary. In the constrained theories, $\lambda$ is a Lagrange multiplier, and by virtue of the constraint $u_{\mu} u^{\mu}=-1$, the factor $\omega u_{\mu} u^{\mu}$ in front of the Ricci scalar can be absorbed into a rescaling of $G$; equivalently, in the constrained theories, we can set $\omega=0$. Note that the possible term $u^{\mu} u^{\nu} R_{\mu \nu}$ can be shown under integration by parts to be equivalent to a linear combination of the terms involving $c_{2}$ and $c_{3}$.

Unconstrained theories were studied during the 1970s as "straw-man" alternatives to GR. In addition to having up to four arbitrary parameters, they also left the magnitude of the vector field arbitrary, since it satisfies a linear homogenous vacuum field equation of the form $\mathcal{L} u^{\mu}=0\left(c_{4}=0\right.$ in all such cases studied). Indeed, this latter fact was one of most serious defects of these theories. Each unconstrained theory studied corresponds to a special case of the action (41), all with $\lambda \equiv 0$ :

\section{General vector-tensor theory; $\omega, \tau, \epsilon, \eta$}

The gravitational Lagrangian for this class of theories had the form $R+\omega u_{\mu} u^{\mu} R+\eta u^{\mu} u^{\nu} R_{\mu \nu}-$ $\epsilon F_{\mu \nu} F^{\mu \nu}+\tau \nabla_{\mu} u_{\nu} \nabla^{\mu} u^{\nu}$, where $F_{\mu \nu}=\nabla_{\mu} u_{\nu}-\nabla_{\nu} u_{\mu}$, corresponding to the values $c_{1}=2 \epsilon-\tau$, $c_{2}=-\eta, c_{1}+c_{2}+c_{3}=-\tau, c_{4}=0$. In these theories $\gamma, \beta, \alpha_{1}$, and $\alpha_{2}$ are complicated functions of the parameters and of $u^{2}=-u^{\mu} u_{\mu}$, while the rest vanish (see TEGP 5.4 [420]).

\section{Will-Nordtvedt theory}

This is the special case $c_{1}=-1, c_{2}=c_{3}=c_{4}=0$. In this theory, the PPN parameters are given by $\gamma=\beta=1, \alpha_{2}=u^{2} /\left(1+u^{2} / 2\right)$, and zero for the rest [431]).

\section{Hellings-Nordtvedt theory; $\omega$}

This is the special case $c_{1}=2, c_{2}=2 \omega, c_{1}+c_{2}+c_{3}=0=c_{4}$. Here $\gamma, \beta, \alpha_{1}$ and $\alpha_{2}$ are complicated functions of the parameters and of $u^{2}$, while the rest vanish [187].

\section{Einstein-Æther theory; $c_{1}, c_{2}, c_{3}, c_{4}$}

The Einstein-Æther theories were motivated in part by a desire to explore possibilities for violations of Lorentz invariance in gravity, in parallel with similar studies in matter interactions, such as the SME. The general class of theories was analyzed by Jacobson and collaborators [204, 274, 205, 147, 163], motivated in part by [230]. Analyzing the post-Newtonian limit, ${ }^{1}$ they were able to infer values of the PPN parameters $\gamma$ and $\beta$ as follows [163]:

$$
\begin{aligned}
\gamma & =1 \\
\beta & =1 \\
\xi & =\alpha_{3}=\zeta_{1}=\zeta_{2}=\zeta_{3}=\zeta_{4}=0, \\
\alpha_{1} & =-\frac{8\left(c_{3}^{2}+c_{1} c_{4}\right)}{2 c_{1}-c_{1}^{2}+c_{3}^{2}}, \\
\alpha_{2} & =-\frac{4\left(c_{3}^{2}+c_{1} c_{4}\right)}{2 c_{1}-c_{1}^{2}+c_{3}^{2}}-\frac{\left(2 c_{13}-c_{14}\right)\left(c_{13}+c_{14}+3 c_{2}\right)}{c_{123}\left(2-c_{14}\right)},
\end{aligned}
$$

where $c_{123}=c_{1}+c_{2}+c_{3}, c_{13}=c_{1}+c_{3}, c_{14}=c_{1}+c_{4}$, subject to the constraints $c_{123} \neq 0$, $c_{14} \neq 2,2 c_{1}-c_{1}^{2}+c_{3}^{2} \neq 0$. By requiring that gravitational-wave modes have real (as opposed to imaginary) frequencies, one can impose the bounds $c_{1} / c_{14} \geq 0$ and $c_{123} / c_{14} \geq 0$. Considerations of positivity of energy impose the constraints $c_{1}>0, c_{14}>0$ and $c_{123}>0$.

1 Note that the minus sign in front the $c_{4}$ term in Eq. (42) compared to that in the references is a result of our convention for the signature of the metric 


\section{Khronometric theory; $\alpha_{K}, \beta_{K}, \lambda_{K}$}

This is the low-energy limit of "Hořava gravity", a proposal for a gravity theory that is powercounting renormalizable [190]. The vector field is required to be hypersurface orthogonal $\left(u^{\alpha} \propto \nabla^{\alpha} T\right.$, where $T$ is a scalar field related to a preferred time direction; equivalently the twist $\omega^{\alpha \beta}=\nabla^{[\alpha} u^{\beta]}+u^{[\alpha} a^{\beta]}$ must vanish, where $a^{\beta}=u^{\mu} \nabla_{\mu} u^{\beta}$ ), so that higher spatial derivative terms could be introduced to effectuate renormalizability. A "healthy" version of the theory [61,62] can be shown to correspond to the values $c_{1}=-\epsilon c_{2}=\lambda_{K}, c_{3}=\beta_{K}+\epsilon$ and $c_{4}=\alpha_{K}+\epsilon$, where the limit $\epsilon \rightarrow \infty$ is to be taken. (The idea is to extract $\epsilon$ times $\omega_{\alpha \beta} \omega^{\alpha \beta}$ from the Einstein-Æther action and let $\epsilon \rightarrow \infty$ to enforce the twist-free condition [203].) In this case $\alpha_{1}$ and $\alpha_{2}$ are given by

$$
\begin{aligned}
\alpha_{1} & =\frac{4\left(\alpha_{K}-2 \beta_{K}\right)}{\beta_{K}-1}, \\
\alpha_{2} & =\frac{1}{2} \alpha_{1}+\frac{\left(\alpha_{K}-2 \beta_{K}\right)\left(\alpha_{K}+\beta_{K}+3 \lambda_{K}\right)}{\left(2-\alpha_{K}\right)\left(\beta_{K}+\lambda_{K}\right)} .
\end{aligned}
$$

\subsubsection{Tensor-vector-scalar (TeVeS) theories}

This class of theories was invented to provide a fully relativistic theory of gravity that could mimic the phenomenological behavior of so-called Modified Newtonian Dynamics (MOND). MOND is a phenomenological mechanism [283] whereby Newton's equation of motion $a=G m / r^{2}$ holds as long as $a$ is large compared to some fundamental scale $a_{0}$, but in a regime where $a<a_{0}$, the equation of motion takes the form $a^{2} / a_{0}=G m / r^{2}$. With such a behavior, the rotational velocity of a particle far from a central mass would have the form $v \sim \sqrt{a r} \sim\left(G m a_{0}\right)^{1 / 4}$, thus reproducing the flat rotation curves observed for spiral galaxies, without invoking a distribution of dark matter.

Devising a relativistic theory that would embody the MOND phenomenology turned out to be no simple matter, and the final result, TeVeS was rather complicated [36]. Furthermore, it was shown to have unexpected singular behavior that was most simply cured by incorporating features of the Einstein-Æther theory [366]. The extended theory is based on an "Einstein" metric $\tilde{g}_{\mu \nu}$, related to the physical metric $g_{\mu \nu}$ by

$$
g_{\mu \nu} \equiv e^{-2 \phi} \tilde{g}_{\mu \nu}-2 u_{\mu} u_{\nu} \sinh (2 \phi),
$$

where $u^{\mu}$ is a vector field, and $\phi$ is a scalar field. The action for gravity is the standard GR action of Eq. (31), but defined using the Einstein metric $\tilde{g}_{\mu \nu}$, while the matter action is that of a standard metric theory, using $g_{\mu \nu}$. These are supplemented by the vector action, given by that of Einstein-Æther theory, Eq. (41), and a scalar action, given by

$$
I_{S}=-\frac{c^{3}}{2 k^{2} \ell^{2} G} \int \mathcal{F}\left(k \ell^{2} h^{\mu \nu} \phi_{, \mu} \phi_{, \nu}\right)(-g)^{1 / 2} d^{4} x,
$$

where $k$ is a constant, $\ell$ is a distance, and $h^{\mu \nu} \equiv \tilde{g}^{\mu \nu}-u^{\mu} u^{\nu}$, indices being raised and lowered using the Einstein metric. The function $\mathcal{F}(y)$ is chosen so that $\mu(y) \equiv d \mathcal{F} / d y$ is unity in the highacceleration, or normal Newtonian and post-Newtonian regimes, and nearly zero in the MOND regime.

The PPN parameters of the theory [346] have the values $\gamma=\beta=1$ and $\xi=\alpha_{3}=\zeta_{i}=0$, while the parameters $\alpha_{1}$ and $\alpha_{2}$ are given by

$$
\begin{aligned}
& \alpha_{1}=\left(\alpha_{1}\right)_{Æ}-16 G \frac{\kappa c_{1}\left(2-c_{14}\right)-c_{3} \sinh 4 \phi_{0}+2\left(1-c_{1}\right) \sinh ^{2} 2 \phi_{0}}{2 c_{1}-c_{1}^{2}+c_{3}^{2}}, \\
& \alpha_{2}=\left(\alpha_{2}\right)_{Æ}-2 G\left(A_{1} \kappa-2 A_{2} \sinh 4 \phi_{0}-A_{3} \sinh ^{2} 2 \phi_{0}\right),
\end{aligned}
$$


where $\left(\alpha_{1}\right)_{Æ}$ and $\left(\alpha_{2}\right)_{\circledast}$ are given by Eqs. (46) and (47), where

$$
\begin{aligned}
& A_{1} \equiv \frac{\left(2 c_{13}-c_{14}\right)^{2}}{c_{123}\left(2-c_{14}\right)}+\frac{4 c_{1}\left(2-c_{14}\right)}{2 c_{1}-c_{1}^{2}+c_{3}^{2}}-\frac{6\left(1+c_{13}-c_{14}\right)}{2-c_{14}}, \\
& A_{2} \equiv \frac{\left(2 c_{13}-c_{14}\right)^{2}}{c_{123}\left(2-c_{14}\right)^{2}}-\frac{4\left(1-c_{1}\right)}{2 c_{1}-c_{1}^{2}+c_{3}^{2}}+\frac{2\left(1-c_{13}\right)}{2-c_{14}}\left(\frac{2}{c_{123}}+\frac{3}{2-c_{14}}\right), \\
& A_{3} \equiv \frac{\left(2 c_{13}-c_{14}\right)^{2}}{c_{123}\left(2-c_{14}\right)^{2}}+\frac{4 c_{3}}{2 c_{1}-c_{1}^{2}+c_{3}^{2}}+\frac{2}{\left(2-c_{14}\right)}\left(\frac{3\left(1-c_{13}\right)}{c_{123}}-\frac{2 c_{13}-c_{14}}{2-c_{14}}\right),
\end{aligned}
$$

where $\kappa \equiv k / 8 \pi$,

$$
G \equiv \frac{1}{2}\left(\frac{2-c_{14}}{1+\kappa\left(2-c_{14}\right)}\right),
$$

and $\phi_{0}$ is the asymptotic value of the scalar field. In the limit $\kappa \rightarrow 0$ and $\phi_{0} \rightarrow 0, \alpha_{1}$ and $\alpha_{2}$ reduce to their Einstein-Æther forms.

However, these PPN parameter values are computed in the limit where the function $\mathcal{F}(y)$ is a linear function of its argument $y=k \ell^{2} h^{\mu \nu} \phi_{, \mu} \phi_{, \nu}$. When one takes into account the fact that the function $\mu(y)=d \mathcal{F} / d y$ must interpolate between unity and zero to reach the MOND regime, it has been found that the dynamics of local systems is more strongly affected by the fields of surrounding matter than was anticipated. This "external field effect" (EFE) [284, 57, 58] produces a quadrupolar contribution to the local Newtonian gravitational potential that depends on the external distribution of matter and on the shape of the function $\mu(y)$, and that can be significantly larger than the galactic tidal contribution. Although the calculations of EFE have been carried out using phenomenological MOND equations, it should be a generic phenomenon, applicable to TeVeS as well. Analysis of the orbit of Saturn using Cassini data has placed interesting constraints on the MOND interpolating function $\mu(y)$ [186].

For thorough reviews of MOND and TeVeS, and their confrontation with the dark-matter paradigm, see [367, 150].

\subsubsection{Quadratic gravity and Chern-Simons theories}

Quadratic gravity is a recent incarnation of an old idea of adding to the action of GR terms quadratic in the Riemann and Ricci tensors or the Ricci scalar, as "effective field theory" models for more fundamental string or quantum gravity theories. The general action for such theories can be written as

$$
\begin{gathered}
I=\int\left[\kappa R+\alpha_{1} f_{1}(\phi) R^{2}+\alpha_{2} f_{2}(\phi) R_{\alpha \beta} R^{\alpha \beta}+\alpha_{3} f_{3}(\phi) R_{\alpha \beta \gamma \delta} R^{\alpha \beta \gamma \delta}+\alpha_{4} f_{4}(\phi)^{*} R R\right. \\
\left.-\frac{\beta}{2}\left(g^{\mu \nu} \partial_{\mu} \phi \partial_{\nu} \phi+2 V(\phi)\right)\right](-g)^{1 / 2} d^{4} x+I_{\mathrm{m}}\left(\psi_{\mathrm{m}}, g_{\mu \nu}\right)
\end{gathered}
$$

where $\kappa=(16 \pi G)^{-1}, \phi$ is a scalar field, $\alpha_{i}$ are dimensionless coupling constants (if the functions $f_{i}(\phi)$ are dimensionless), and $\beta$ is a constant whose dimension depends on that of $\phi$, and where ${ }^{*} R R \equiv{ }^{*} R^{\alpha}{ }_{\beta}{ }^{\gamma \delta} R^{\beta}{ }_{\alpha \gamma \delta}$, where ${ }^{*} R^{\alpha}{ }_{\beta}{ }^{\gamma \delta} \equiv \frac{1}{2} \epsilon^{\gamma \delta \rho \sigma} R_{\beta \rho \sigma}^{\alpha}$ is the dual Riemann tensor.

One challenge inherent in these theories is to find an argument or a mechanism that evades making the natural choice for each of the $\alpha$ parameters to be of order unity. Such a choice makes the effects of the additional terms essentially unobservable in most laboratory or astrophysical situations because of the enormous scale of $\kappa \propto 1 / \ell_{\text {Planck }}^{2}$ in the leading term. This class of theories is too vast and diffuse to cover in this review, and no comprehensive review is available, to our knowledge. 
Chern-Simons gravity is the special case of this class of theories in which only the parityviolating term ${ }^{*} R R$ is present $\left(\alpha_{1}=\alpha_{2}=\alpha_{3}=0\right)$ [201]. It can arise in various anomaly cancellation schemes in the standard model of particle physics, in cancelling the Green-Schwarz anomaly in string theory, or in effective field theories of inflation [408]. It can also arise in loop quantum gravity $[382,276]$. The action in this case is given by

$$
I=\int\left[\kappa R+\frac{\alpha}{4} \phi^{*} R R-\frac{\beta}{2}\left(g^{\mu \nu} \partial_{\mu} \phi \partial_{\nu} \phi+2 V(\phi)\right)\right](-g)^{1 / 2} d^{4} x+I_{\mathrm{m}}\left(\psi_{\mathrm{m}}, g_{\mu \nu}\right),
$$

where $\alpha$ and $\beta$ are coupling constants with dimensions $\ell^{A}$, and $\ell^{2 A-2}$, assuming that the scalar field has dimensions $\ell^{-A}$.

There are two different versions of Chern-Simons theory, a non-dynamical version in which $\beta=0$, so that $\phi$, given a priori as some specified function of spacetime, plays the role of a Lagrange multiplier enforcing the constraint ${ }^{*} R R=0$, and a dynamical version, in which $\beta \neq 0$.

The PPN parameters for a non-dynamical version of the theory with $\alpha=\kappa$ and $\beta=0$ are identical to those of GR; however, there is an additional, parity-even potential in the $g_{0 i}$ component of the metric that does not appear in the standard PPN framework, given by

$$
\delta g_{0 i}=2 \frac{d \phi}{d t}(\nabla \times \mathbf{V})_{i} .
$$

Unfortunately, the non-dynamical version has been shown to be unstable [137], while the dynamical version is sufficiently complex that its observable consequences have been analyzed for only special situations [6, 444]. Alexander and Yunes [5] give a thorough review of Chern-Simons gravity.

Einstein-Dilaton-Gauss-Bonnet gravity is another special case, in which the Chern-Simons term is neglected $\left(\alpha_{4}=0\right)$, and the three other curvature-squared terms collapse to the GaussBonnet invariant, $R^{2}-4 R_{\alpha \beta} R^{\alpha \beta}+R_{\alpha \beta \gamma \delta} R^{\alpha \beta \gamma \delta}$, i.e. $f_{1}(\phi)=f_{2}(\phi)=f_{3}(\phi)$ and $\alpha_{1}=-\alpha_{2} / 4=\alpha_{3}$ (see $[292,314])$.

\subsubsection{Massive gravity}

Massive gravity theories attempt to give the putative "graviton" a mass. The simplest attempt to implement this in a ghost-free manner suffers from the so-called van Dam-Veltman-Zakharov (vDVZ) discontinuity $[398,453]$. Because of the 3 additional helicity states available to the massive spin-2 graviton, the limit of small graviton mass does not coincide with pure GR, and the predicted perihelion advance, for example, violates experiment. A model theory by Visser [401] attempts to circumvent the vDVZ problem by introducing a non-dynamical flat-background metric. This theory is truly continuous with GR in the limit of vanishing graviton mass; on the other hand, its observational implications have been only partially explored. Braneworld scenarios predict a tower or a continuum of massive gravitons, and may avoid the vDVZ discontinuity, although the full details are still a work in progress [125, 96]. Attempts to avert the vDVZ problem involve treating non-linear aspects of the theory at the fundamental level; many models incorporate a second tensor field in addition to the metric. For recent reviews, see [188, 123], and a focus issue in Vol. 30, Number 18 of Classical and Quantum Gravity. 


\section{Tests of Post-Newtonian Gravity}

\subsection{Tests of the parameter $\gamma$}

With the PPN formalism in hand, we are now ready to confront gravitation theories with the results of solar-system experiments. In this section we focus on tests of the parameter $\gamma$, consisting of the deflection of light and the time delay of light.

\subsubsection{The deflection of light}

A light ray (or photon) which passes the Sun at a distance $d$ is deflected by an angle

$$
\delta \theta=\frac{1}{2}(1+\gamma) \frac{4 M_{\odot}}{d} \frac{1+\cos \Phi}{2}
$$

(TEGP 7.1 [420]), where $M_{\odot}$ is the mass of the Sun and $\Phi$ is the angle between the Earth-Sun line and the incoming direction of the photon (see Figure 4 ). For a grazing ray, $d \approx R_{\odot}, \Phi \approx 0$, and

$$
\delta \theta \approx \frac{1}{2}(1+\gamma) 1 .^{\prime \prime} 7505
$$

independent of the frequency of light. Another, more useful expression gives the change in the relative angular separation between an observed source of light and a nearby reference source as both rays pass near the Sun:

$$
\delta \theta=\frac{1}{2}(1+\gamma)\left[-\frac{4 M_{\odot}}{d} \cos \chi+\frac{4 M_{\odot}}{d_{\mathrm{r}}}\left(\frac{1+\cos \Phi_{\mathrm{r}}}{2}\right)\right]
$$

where $d$ and $d_{\mathrm{r}}$ are the distances of closest approach of the source and reference rays respectively, $\Phi_{\mathrm{r}}$ is the angular separation between the Sun and the reference source, and $\chi$ is the angle between the Sun-source and the Sun-reference directions, projected on the plane of the sky (see Figure 4). Thus, for example, the relative angular separation between the two sources may vary if the line of sight of one of them passes near the Sun $\left(d \sim R_{\odot}, d_{\mathrm{r}} \gg d, \chi\right.$ varying with time).

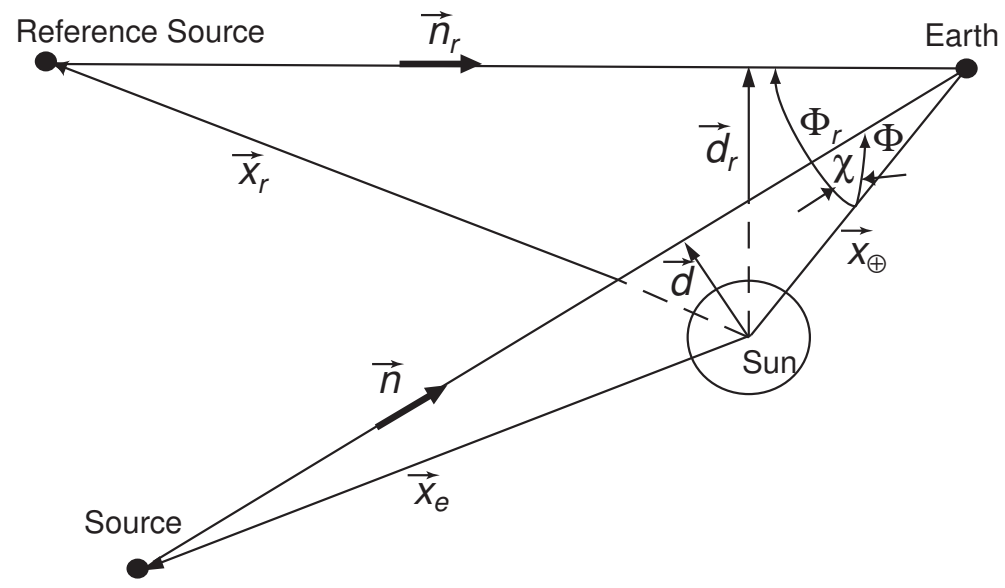

Figure 4: Geometry of light deflection measurements.

It is interesting to note that the classic derivations of the deflection of light that use only the corpuscular theory of light (Cavendish 1784, von Soldner 1803 [416]), or the principle of equivalence 
(Einstein 1911), yield only the "1/2" part of the coefficient in front of the expression in Eq. (61). But the result of these calculations is the deflection of light relative to local straight lines, as established for example by rigid rods; however, because of space curvature around the Sun, determined by the PPN parameter $\gamma$, local straight lines are bent relative to asymptotic straight lines far from the Sun by just enough to yield the remaining factor " $\gamma / 2$ ". The first factor " $1 / 2$ " holds in any metric theory, the second " $\gamma / 2$ " varies from theory to theory. Thus, calculations that purport to derive the full deflection using the equivalence principle alone are incorrect.

The prediction of the full bending of light by the Sun was one of the great successes of Einstein's GR. Eddington's confirmation of the bending of optical starlight observed during a solar eclipse in the first days following World War I helped make Einstein famous. However, the experiments of Eddington and his co-workers had only 30 percent accuracy (for a recent re-evaluation of Eddington's conclusions, see [215]). Succeeding experiments were not much better: the results were scattered between one half and twice the Einstein value (see Figure 5), and the accuracies were low. For a history of this period see [95].

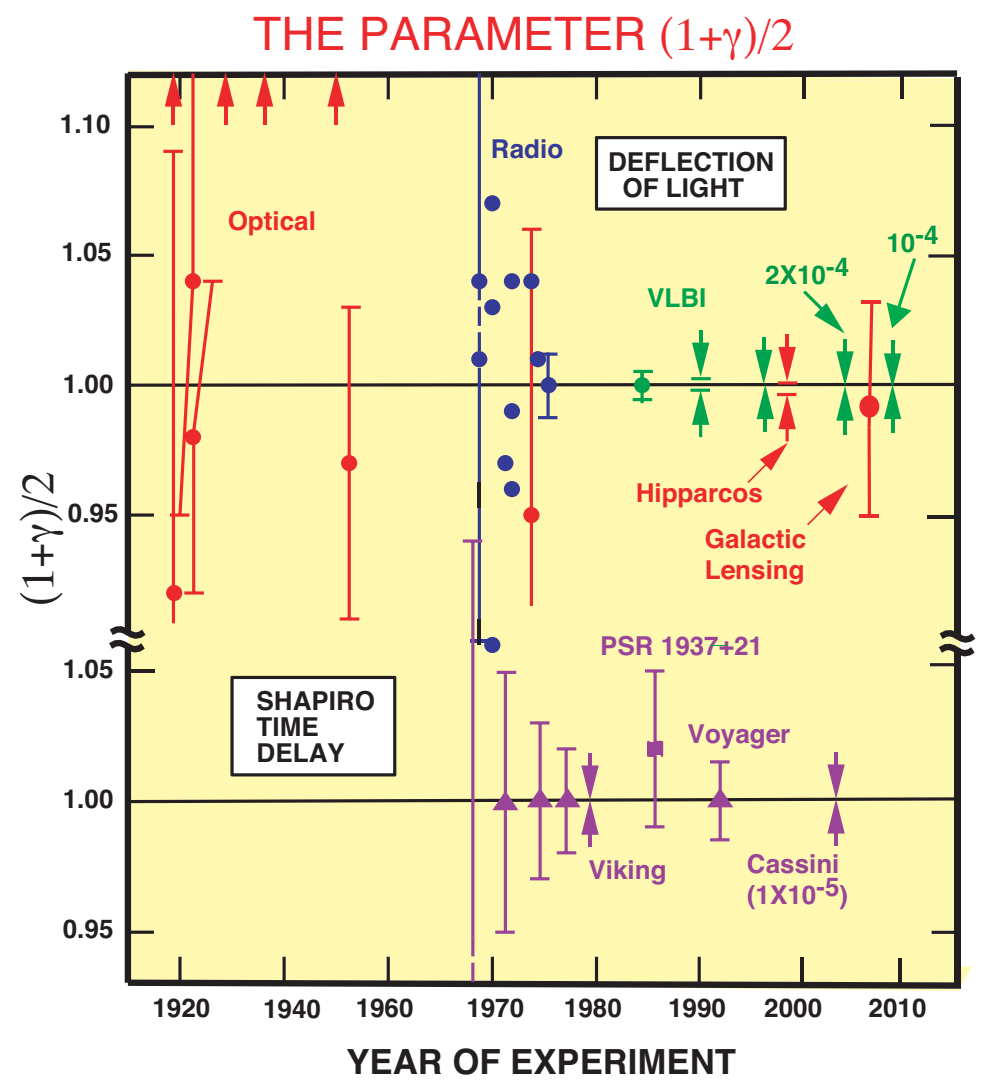

Figure 5: Measurements of the coefficient $(1+\gamma) / 2$ from light deflection and time delay measurements. Its GR value is unity. The arrows at the top denote anomalously large values from early eclipse expeditions. The Shapiro time-delay measurements using the Cassini spacecraft yielded an agreement with GR to $10^{-3}$ percent, and VLBI light deflection measurements have reached 0.01 percent. Hipparcos denotes the optical astrometry satellite, which reached 0.1 percent.

However, the development of radio interferometery, and later of very-long-baseline radio interferometry (VLBI), produced greatly improved determinations of the deflection of light. These tech- 
niques now have the capability of measuring angular separations and changes in angles to accuracies better than 100 microarcseconds. Early measurements took advantage of a series of heavenly coincidences: Each year, groups of strong quasistellar radio sources pass very close to the Sun (as seen from the Earth), including the group 3C273, 3C279, and 3C48, and the group 0111+02, 0119+11, and $0116+08$. As the Earth moves in its orbit, changing the lines of sight of the quasars relative to the Sun, the angular separation $\delta \theta$ between pairs of quasars varies (see Eq. (63)). The time variation in the quantities $d, d_{\mathrm{r}}, \chi$, and $\Phi_{\mathrm{r}}$ in Eq. (63) is determined using an accurate ephemeris for the Earth and initial directions for the quasars, and the resulting prediction for $\delta \theta$ as a function of time is used as a basis for a least-squares fit of the measured $\delta \theta$, with one of the fitted parameters being the coefficient $\frac{1}{2}(1+\gamma)$. A number of measurements of this kind over the period 1969-1975 yielded an accurate determination of the coefficient $\frac{1}{2}(1+\gamma)$, or equivalently $\gamma-1$. A 1995 VLBI measurement using 3C273 and 3C279 yielded $\gamma-1=(-8 \pm 34) \times 10^{-4}$ [243], while a 2009 measurement using the VLBA targeting the same two quasars plus two other nearby radio sources yielded $\gamma-1=(-2 \pm 3) \times 10^{-4}[161]$.

In recent years, transcontinental and intercontinental VLBI observations of quasars and radio galaxies have been made primarily to monitor the Earth's rotation ("VLBI" in Figure 5). These measurements are sensitive to the deflection of light over almost the entire celestial sphere (at $90^{\circ}$ from the Sun, the deflection is still 4 milliarcseconds). A 2004 analysis of almost 2 million VLBI observations of 541 radio sources, made by 87 VLBI sites yielded $(1+\gamma) / 2=0.99992 \pm 0.00023$, or equivalently, $\gamma-1=(-1.7 \pm 4.5) \times 10^{-4}$ [363]. Analyses that incorporated data through 2010 yielded $\gamma-1=(-0.8 \pm 1.2) \times 10^{-4}[237,238]$.

Analysis of observations made by the Hipparcos optical astrometry satellite yielded a test at the level of 0.3 percent [165]. A VLBI measurement of the deflection of light by Jupiter was reported in 1991; the predicted deflection of about 300 microarcseconds was seen with about 50 percent accuracy [389].

Finally, a remarkable measurement of $\gamma$ on galactic scales was reported in 2006 [64]. It used data on gravitational lensing by 15 elliptical galaxies, collected by the Sloan Digital Sky Survey. The Newtonian potential $U$ of each lensing galaxy (including the contribution from dark matter) was derived from the observed velocity dispersion of stars in the galaxy. Comparing the observed lensing with the lensing predicted by the models provided a 10 percent bound on $\gamma$, in agreement with general relativity. Unlike the much tighter bounds described previously, which were obtained on the scale of the solar system, this bound was obtained on a galactic scale.

The results of light-deflection measurements are summarized in Figure 5.

\subsubsection{The time delay of light}

A radar signal sent across the solar system past the Sun to a planet or satellite and returned to the Earth suffers an additional non-Newtonian delay in its round-trip travel time, given by (see Figure 4)

$$
\delta t=2(1+\gamma) M_{\odot} \ln \left(\frac{\left(r_{\oplus}+\mathbf{x}_{\oplus} \cdot \mathbf{n}\right)\left(r_{\mathrm{e}}-\mathbf{x}_{\mathrm{e}} \cdot \mathbf{n}\right)}{d^{2}}\right)
$$

where $\mathbf{x}_{\mathrm{e}}\left(\mathbf{x}_{\oplus}\right)$ are the vectors, and $r_{\mathrm{e}}\left(r_{\oplus}\right)$ are the distances from the Sun to the source (Earth), respectively (TEGP $7.2[420]$ ). For a ray which passes close to the Sun,

$$
\delta t \approx \frac{1}{2}(1+\gamma)\left[240-20 \ln \left(\frac{d^{2}}{r}\right)\right] \mu \mathrm{s},
$$

where $d$ is the distance of closest approach of the ray in solar radii, and $r$ is the distance of the planet or satellite from the Sun, in astronomical units.

In the two decades following Irwin Shapiro's 1964 discovery of this effect as a theoretical consequence of GR, several high-precision measurements were made using radar ranging to targets 
passing through superior conjunction. Since one does not have access to a "Newtonian" signal against which to compare the round-trip travel time of the observed signal, it is necessary to do a differential measurement of the variations in round-trip travel times as the target passes through superior conjunction, and to look for the logarithmic behavior of Eq. (65). In order to do this accurately however, one must take into account the variations in round-trip travel time due to the orbital motion of the target relative to the Earth. This is done by using radar-ranging (and possibly other) data on the target taken when it is far from superior conjunction (i.e., when the time-delay term is negligible) to determine an accurate ephemeris for the target, using the ephemeris to predict the PPN coordinate trajectory $\mathbf{x}_{\mathrm{e}}(t)$ near superior conjunction, then combining that trajectory with the trajectory of the Earth $\mathbf{x}_{\oplus}(t)$ to determine the Newtonian round-trip time and the logarithmic term in Eq. (65). The resulting predicted round-trip travel times in terms of the unknown coefficient $\frac{1}{2}(1+\gamma)$ are then fit to the measured travel times using the method of least-squares, and an estimate obtained for $\frac{1}{2}(1+\gamma)$.

The targets employed included planets, such as Mercury or Venus, used as passive reflectors of the radar signals ("passive radar"), and artificial satellites, such as Mariners 6 and 7, Voyager 2, the Viking Mars landers and orbiters, and the Cassini spacecraft to Saturn, used as active retransmitters of the radar signals ("active radar").

The results for the coefficient $\frac{1}{2}(1+\gamma)$ of all radar time-delay measurements performed to date (including a measurement of the one-way time delay of signals from the millisecond pulsar PSR 1937+21) are shown in Figure 5 (see TEGP 7.2 [420] for discussion and references). The 1976 Viking experiment resulted in a 0.1 percent measurement [333].

A significant improvement was reported in 2003 from Doppler tracking of the Cassini spacecraft while it was on its way to Saturn [44], with a result $\gamma-1=(2.1 \pm 2.3) \times 10^{-5}$. This was made possible by the ability to do Doppler measurements using both X-band $(7175 \mathrm{MHz})$ and Ka-band (34316 MHz) radar, thereby significantly reducing the dispersive effects of the solar corona. Note that with Doppler measurements, one is essentially measuring the time derivative of the Shapiro delay. In addition, the 2002 superior conjunction of Cassini was particularly favorable: with the spacecraft at 8.43 astronomical units from the Sun, the distance of closest approach of the radar signals to the Sun was only $1.6 R_{\odot}$.

From the results of the Cassini experiment, we can conclude that the coefficient $\frac{1}{2}(1+\gamma)$ must be within at most 0.0012 percent of unity. Massless scalar-tensor theories must have $\omega>40000$ to be compatible with this constraint.

\subsubsection{Shapiro time delay and the speed of gravity}

In 2001, Kopeikin [221] suggested that a measurement of the time delay of light from a quasar as the light passed by the planet Jupiter could be used to measure the speed of the gravitational interaction. He argued that, since Jupiter is moving relative to the solar system, and since gravity propagates with a finite speed, the gravitational field experienced by the light ray should be affected by gravity's speed, since the field experienced at one time depends on the location of the source a short time earlier, depending on how fast gravity propagates. According to his calculations, there should be a post ${ }^{1 / 2}$-Newtonian correction to the normal Shapiro time-delay formula (64) which depends on the velocity of Jupiter and on the velocity of gravity. On September 8, 2002, Jupiter passed almost in front of a quasar, and Kopeikin and Fomalont made precise measurements of the Shapiro delay with picosecond timing accuracy, and claimed to have measured the correction term to about 20 percent [162, 226, 222, 223].

However, several authors pointed out that this 1.5PN effect does not depend on the speed of propagation of gravity, but rather only depends on the speed of light [24, 425, 348, 73, 349]. Intuitively, if one is working to only first order in $v / c$, then all that counts is the uniform motion of the planet Jupiter (its acceleration about the Sun contributes a higher-order, unmeasurably small 
effect). But if that is the case, then the principle of relativity says that one can view things from the rest frame of Jupiter. In this frame, Jupiter's gravitational field is static, and the speed of propagation of gravity is irrelevant. A detailed post-Newtonian calculation of the effect was done using a variant of the PPN framework, in a class of theories in which the speed of gravity could be different from that of light [425], and found explicitly that, at first order in $v / c$, the effect depends on the speed of light, not the speed of gravity, in line with intuition. Effects dependent upon the speed of gravity show up only at higher order in $v / c$. Kopeikin gave a number of arguments in opposition to this interpretation [223, 225, 224]. On the other hand, the $v / c$ correction term does show a dependence on the PPN parameter $\alpha_{1}$, which could be non-zero in theories of gravity with a differing speed $c_{\mathrm{g}}$ of gravity (see Eq. (7) of [425]). But existing tight bounds on $\alpha_{1}$ from other experiments (see Table 4) already far exceed the capability of the Jupiter VLBI experiment.

Table 4: Current limits on the PPN parameters.

\begin{tabular}{llcl}
\hline Parameter & \multicolumn{1}{c}{ Effect } & \multicolumn{1}{c}{ Limit } & \multicolumn{1}{c}{ Remarks } \\
\hline$\gamma-1$ & time delay & $2.3 \times 10^{-5}$ & Cassini tracking \\
& light deflection & $2 \times 10^{-4}$ & VLBI \\
$\beta-1$ & perihelion shift & $8 \times 10^{-5}$ & $J_{2 \odot}=(2.2 \pm 0.1) \times 10^{-7}$ \\
& Nordtvedt effect & $2.3 \times 10^{-4}$ & $\eta_{\mathrm{N}}=4 \beta-\gamma-3$ assumed \\
$\xi$ & spin precession & $4 \times 10^{-9}$ & millisecond pulsars \\
$\alpha_{1}$ & orbital polarization & $10^{-4}$ & Lunar laser ranging \\
& & $4 \times 10^{-5}$ & PSR J1738+0333 \\
$\alpha_{2}$ & spin precession & $2 \times 10^{-9}$ & millisecond pulsars \\
$\alpha_{3}$ & pulsar acceleration & $4 \times 10^{-20}$ & pulsar $\dot{P}$ statistics \\
$\zeta_{1}$ & $\quad-$ & $2 \times 10^{-2}$ & combined PPN bounds \\
$\zeta_{2}$ & binary acceleration & $4 \times 10^{-5}$ & $\ddot{P}_{\mathrm{p}}$ for PSR 1913+16 \\
$\zeta_{3}$ & Newton's 3rd law & $10^{-8}$ & lunar acceleration \\
$\zeta_{4}$ & - & - & not independent [see Eq. (73)] \\
\hline
\end{tabular}

\subsection{The perihelion shift of Mercury}

The explanation of the anomalous perihelion shift of Mercury's orbit was another of the triumphs of GR. This had been an unsolved problem in celestial mechanics for over half a century, since the announcement by Le Verrier in 1859 that, after the perturbing effects of the planets on Mercury's orbit had been accounted for, and after the effect of the precession of the equinoxes on the astronomical coordinate system had been subtracted, there remained in the data an unexplained advance in the perihelion of Mercury. The modern value for this discrepancy is 43 arcseconds per century. A number of ad hoc proposals were made in an attempt to account for this excess, including, among others, the existence of a new planet Vulcan near the Sun, a ring of planetoids, a solar quadrupole moment and a deviation from the inverse-square law of gravitation, but none was successful. General relativity accounted for the anomalous shift in a natural way without disturbing the agreement with other planetary observations.

The predicted advance per orbit $\Delta \tilde{\omega}$, including both relativistic PPN contributions and the Newtonian contribution resulting from a possible solar quadrupole moment, is given by

$$
\Delta \tilde{\omega}=\frac{6 \pi m}{p}\left(\frac{1}{3}(2+2 \gamma-\beta)+\frac{1}{6}\left(2 \alpha_{1}-\alpha_{2}+\alpha_{3}+2 \zeta_{2}\right) \eta+\frac{J_{2} R^{2}}{2 m p}\right),
$$


where $m \equiv m_{1}+m_{2}$ and $\eta \equiv m_{1} m_{2} / m^{2}$ are the total mass and dimensionless reduced mass of the two-body system respectively; $p \equiv a\left(1-e^{2}\right)$ is the semi-latus rectum of the orbit, with the semi-major axis $a$ and the eccentricity $e ; R$ is the mean radius of the oblate body; and $J_{2}$ is a dimensionless measure of its quadrupole moment, given by $J_{2}=(C-A) / m_{1} R^{2}$, where $C$ and $A$ are the moments of inertia about the body's rotation and equatorial axes, respectively (for details of the derivation see TEGP 7.3 [420]). We have ignored preferred-frame and galaxy-induced contributions to $\Delta \tilde{\omega}$; these are discussed in TEGP 8.3 [420].

The first term in Eq. (66) is the classical relativistic perihelion shift, which depends upon the PPN parameters $\gamma$ and $\beta$. The second term depends upon the ratio of the masses of the two bodies; it is zero in any fully conservative theory of gravity $\left(\alpha_{1} \equiv \alpha_{2} \equiv \alpha_{3} \equiv \zeta_{2} \equiv 0\right)$; it is also negligible for Mercury, since $\eta \approx m_{\mathrm{Merc}} / M_{\odot} \approx 2 \times 10^{-7}$. We shall drop this term henceforth.

The third term depends upon the solar quadrupole moment $J_{2}$. For a Sun that rotates uniformly with its observed surface angular velocity, so that the quadrupole moment is produced by centrifugal flattening, one may estimate $J_{2}$ to be $\sim 1 \times 10^{-7}$. This actually agrees reasonably well with values inferred from rotating solar models that are in accord with observations of the normal modes of solar oscillations (helioseismology); the latest inversions of helioseismology data give $J_{2}=(2.2 \pm 0.1) \times 10^{-7}[275,17]$; for a review of measurements of the solar quadrupole moment, see [344]. Substituting standard orbital elements and physical constants for Mercury and the Sun we obtain the rate of perihelion shift $\dot{\tilde{\omega}}$, in seconds of arc per century,

$$
\dot{\tilde{\omega}}=42 .^{\prime \prime} 98\left(\frac{1}{3}(2+2 \gamma-\beta)+3 \times 10^{-4} \frac{J_{2}}{10^{-7}}\right) .
$$

The most recent fits to planetary data include data from the Messenger spacecraft that orbited Mercury, thereby significantly improving knowledge of its orbit. Adopting the Cassini bound on $\gamma$ a priori, these analyses yield a bound on $\beta$ given by $\beta-1=(-4.1 \pm 7.8) \times 10^{-5}$. Further analysis could push this bound even lower [152, 399], although knowledge of $J_{2}$ would have to improve simultaneously. A slightly weaker bound $\beta-1=(0.4 \pm 2.4) \times 10^{-4}$ from the perihelion advance of Mars (again adopting the Cassini bound on $\gamma$ ) was obtained by exploiting data from the Mars Reconnaissance Orbiter [220]

Laser tracking of the Earth-orbiting satellite LAGEOS II led to a measurement of its relativistic perigee precession (3.4 arcseconds per year) in agreement with GR to two percent [262, 263] (note that the second paper contains an improved assessment of systematic errors).

\subsection{Tests of the strong equivalence principle}

The next class of solar-system experiments that test relativistic gravitational effects may be called tests of the strong equivalence principle (SEP). In Section 3.1.2 we pointed out that many metric theories of gravity (perhaps all except GR) can be expected to violate one or more aspects of SEP. Among the testable violations of SEP are a violation of the weak equivalence principle for gravitating bodies that leads to perturbations in the Earth-Moon orbit, preferred-location and preferred-frame effects in the locally measured gravitational constant that could produce observable geophysical effects, and possible variations in the gravitational constant over cosmological timescales.

\subsubsection{The Nordtvedt effect and the lunar Eötvös experiment}

In a pioneering calculation using his early form of the PPN formalism, Nordtvedt [304] showed that many metric theories of gravity predict that massive bodies violate the weak equivalence principle - that is, fall with different accelerations depending on their gravitational self-energy. Dicke [342] argued that such an effect would occur in theories with a spatially varying gravitational constant, 
such as scalar-tensor gravity. For a spherically symmetric body, the acceleration from rest in an external gravitational potential $U$ has the form

$$
\begin{aligned}
\mathbf{a} & =\frac{m_{\mathrm{p}}}{m} \nabla U, \\
\frac{m_{\mathrm{p}}}{m} & =1-\eta_{\mathrm{N}} \frac{E_{\mathrm{g}}}{m}, \\
\eta_{\mathrm{N}} & =4 \beta-\gamma-3-\frac{10}{3} \xi-\alpha_{1}+\frac{2}{3} \alpha_{2}-\frac{2}{3} \zeta_{1}-\frac{1}{3} \zeta_{2},
\end{aligned}
$$

where $E_{\mathrm{g}}$ is the negative of the gravitational self-energy of the body $\left(E_{\mathrm{g}}>0\right)$. This violation of the massive-body equivalence principle is known as the "Nordtvedt effect". The effect is absent in $\operatorname{GR}\left(\eta_{\mathrm{N}}=0\right)$ but present in scalar-tensor theory $\left(\eta_{\mathrm{N}}=1 /(2+\omega)+4 \lambda\right)$. The existence of the Nordtvedt effect does not violate the results of laboratory Eötvös experiments, since for laboratory-sized objects $E_{\mathrm{g}} / m \leq 10^{-27}$, far below the sensitivity of current or future experiments. However, for astronomical bodies, $E_{\mathrm{g}} / m$ may be significant $\left(3.6 \times 10^{-6}\right.$ for the Sun, $10^{-8}$ for Jupiter, $4.6 \times 10^{-10}$ for the Earth, $0.2 \times 10^{-10}$ for the Moon). If the Nordtvedt effect is present $\left(\eta_{\mathrm{N}} \neq 0\right)$ then the Earth should fall toward the Sun with a slightly different acceleration than the Moon. This perturbation in the Earth-Moon orbit leads to a polarization of the orbit that is directed toward the Sun as it moves around the Earth-Moon system, as seen from Earth. This polarization represents a perturbation in the Earth-Moon distance of the form

$$
\delta r=13.1 \eta_{\mathrm{N}} \cos \left(\omega_{0}-\omega_{\mathrm{s}}\right) t[\mathrm{~m}],
$$

where $\omega_{0}$ and $\omega_{\mathrm{s}}$ are the angular frequencies of the orbits of the Moon and Sun around the Earth (see TEGP 8.1 [420] for detailed derivations and references; for improved calculations of the numerical coefficient, see [309, 120]).

Since August 1969, when the first successful acquisition was made of a laser signal reflected from the Apollo 11 retroreflector on the Moon, the LLR experiment has made regular measurements of the round-trip travel times of laser pulses between a network of observatories and the lunar retroreflectors, with accuracies that are approaching the $5 \mathrm{ps}(1 \mathrm{~mm})$ level. These measurements are fit using the method of least-squares to a theoretical model for the lunar motion that takes into account perturbations due to the Sun and the other planets, tidal interactions, and post-Newtonian gravitational effects. The predicted round-trip travel times between retroreflector and telescope also take into account the librations of the Moon, the orientation of the Earth, the location of the observatories, and atmospheric effects on the signal propagation. The "Nordtvedt" parameter $\eta_{\mathrm{N}}$ along with several other important parameters of the model are then estimated in the least-squares method. For a review of lunar laser ranging, see [277].

Numerous ongoing analyses of the data find no evidence, within experimental uncertainty, for the Nordtvedt effect [436, 437] (for earlier results see [132, 435, 295]). These results represent a limit on a possible violation of WEP for massive bodies of about 1.4 parts in $10^{13}$ (compare Figure 1).

However, at this level of precision, one cannot regard the results of LLR as a "clean" test of SEP until one eliminates the possibility of a compensating violation of WEP for the two bodies, because the chemical compositions of the Earth and Moon differ in the relative fractions of iron and silicates. To this end, the Eöt-Wash group carried out an improved test of WEP for laboratory bodies whose chemical compositions mimic that of the Earth and Moon. The resulting bound of 1.4 parts in $10^{13}[29,1]$ from composition effects reduces the ambiguity in the LLR bound, and establishes the firm SEP test at the level of about 2 parts in $10^{13}$. These results can be summarized by the Nordtvedt parameter bound $\left|\eta_{\mathrm{N}}\right|=(4.4 \pm 4.5) \times 10^{-4}$.

APOLLO, the Apache Point Observatory for Lunar Laser-ranging Operation, a joint effort by researchers from the Universities of Washington, Seattle, and California, San Diego, has achieved 
$\mathrm{mm}$ ranging precision using enhanced laser and telescope technology, together with a good, highaltitude site in New Mexico. However models of the lunar orbit must be improved in parallel in order to achieve an order-of-magnitude improvement in the test of the Nordtvedt effect [298]. This effort will be aided by the fortuitous 2010 discovery by the Lunar Reconnaissance Orbiter of the precise landing site of the Soviet Lunokhod I rover, which deployed a retroreflector in 1970. Its uncertain location made it effectively "lost" to lunar laser ranging for almost 40 years. Its location on the lunar surface will make it useful in improving models of the lunar libration [297].

In GR, the Nordtvedt effect vanishes; at the level of several centimeters and below, a number of non-null general relativistic effects should be present [309].

Tests of the Nordtvedt effect for neutron stars have also been carried out using a class of systems known as wide-orbit binary millisecond pulsars (WBMSP), which are pulsar-white-dwarf binary systems with small orbital eccentricities. In the gravitational field of the galaxy, a nonzero Nordtvedt effect can induce an apparent anomalous eccentricity pointed toward the galactic center [118], which can be bounded using statistical methods, given enough WBMSPs (see [374] for a review and references). Using data from 21 WBMSPs, including recently discovered highly circular systems, Stairs et al. [375] obtained the bound $\Delta<5.6 \times 10^{-3}$, where $\Delta=\eta_{\mathrm{N}}\left(E_{\mathrm{g}} / M\right)_{\mathrm{NS}}$. Because $\left(E_{\mathrm{g}} / M\right)_{\mathrm{NS}} \sim 0.1$ for typical neutron stars, this bound does not compete with the bound on $\eta_{\mathrm{N}}$ from LLR; on the other hand, it does test SEP in the strong-field regime because of the presence of the neutron stars. The 2013 discovery of a millisecond pulsar in orbit with two white dwarfs in very circular, coplanar orbits [332] may lead to a test of the Nordvedt effect in the strong-field regime that surpasses the precision of lunar laser ranging by a substantial factor (see Section 6.2).

\subsubsection{Preferred-frame and preferred-location effects}

Some theories of gravity violate SEP by predicting that the outcomes of local gravitational experiments may depend on the velocity of the laboratory relative to the mean rest frame of the universe (preferred-frame effects) or on the location of the laboratory relative to a nearby gravitating body (preferred-location effects). In the post-Newtonian limit, preferred-frame effects are governed by the values of the PPN parameters $\alpha_{1}, \alpha_{2}$, and $\alpha_{3}$, and some preferred-location effects are governed by $\xi$ (see Table 2).

The most important such effects are variations and anisotropies in the locally-measured value of the gravitational constant which lead to anomalous Earth tides and variations in the Earth's rotation rate, anomalous contributions to the orbital dynamics of planets and the Moon, selfaccelerations of pulsars, anomalous torques on the Sun that would cause its spin axis to be randomly oriented relative to the ecliptic (see TEGP 8.2, 8.3, 9.3, and 14.3 (c) [420]), and torques on spinning pulsars that could be seen in variations in their pulse profiles.

A tight bound on $\alpha_{3}$ of $4 \times 10^{-20}$ was obtained by placing limits on anomalous eccentricities in the orbits of a number of binary millisecond pulsars [37,375]. The best bound on $\alpha_{1}$, comes from the orbit of the pulsar-white-dwarf system J1738+0333 [359]. Early bounds on on $\alpha_{2}$ and $\xi$ came from searches for variations induced by an anisotropy in $G$ on the acceleration of gravity on Earth using gravimeters, and (in the case of $\alpha_{2}$ ) from limiting the effects of any anomalous torque on the spinning Sun over the age of the solar system. Today the best bounds on $\alpha_{2}$ and $\xi$ come from bounding torques on the solitary millisecond pulsars B1937+21 and J1744-1134 [358, 360]. Because these later bounds involve systems with strong internal gravity of the neutron stars, they should strictly speaking be regarded as bounds on "strong field" analogues of the PPN parameters. Here we will treat them as bounds on the standard PPN parameters, as shown in Table 4.

\subsubsection{Constancy of the Newtonian gravitational constant}

Most theories of gravity that violate SEP predict that the locally measured Newtonian gravitational constant may vary with time as the universe evolves. For the scalar-tensor theories listed in Table 3, 
the predictions for $\dot{G} / G$ can be written in terms of time derivatives of the asymptotic scalar field. Where $G$ does change with cosmic evolution, its rate of variation should be of the order of the expansion rate of the universe, i.e., $\dot{G} / G \sim H_{0}$, where $H_{0}$ is the Hubble expansion parameter, given by $H_{0}=69 \pm 1 \mathrm{~km} \mathrm{~s}^{-1} \mathrm{Mpc}^{-1}=7 \times 10^{-11} \mathrm{yr}^{-1}$ [39].

Several observational constraints can be placed on $\dot{G} / G$, one kind coming from bounding the present rate of variation, another from bounding a difference between the present value and a past value. The first type of bound typically comes from LLR measurements, planetary radar-ranging measurements, and pulsar timing data. The second type comes from studies of the evolution of the Sun, stars and the Earth, Big-Bang nucleosynthesis, and analyses of ancient eclipse data. Recent results are shown in Table 5 .

Table 5: Constancy of the gravitational constant. For binary-pulsar data, the bounds are dependent upon the theory of gravity in the strong-field regime and on neutron star equation of state. Big-Bang nucleosynthesis bounds assume specific form for time dependence of $G$.

\begin{tabular}{lcl}
\hline Method & $\begin{array}{c}\dot{G} / G \\
\left(10^{-13} \mathrm{yr}^{-1}\right)\end{array}$ & Reference \\
\hline Mars ephemeris & $0.1 \pm 1.6$ & {$[220]$} \\
Lunar laser ranging & $4 \pm 9$ & {$[436]$} \\
Binary \& millisecond pulsars & $-7 \pm 33$ & {$[126,242]$} \\
Helioseismology & $0 \pm 16$ & {$[180]$} \\
Big-Bang nucleosynthesis & $0 \pm 4$ & {$[94,31]$} \\
\hline
\end{tabular}

The best limits on a current $\dot{G} / G$ come from improvements in the ephemeris of Mars using range and Doppler data from the Mars Global Surveyor (1998-2006), Mars Odyssey (2002-2008), and Mars Reconnaissance Orbiter (2006-2008), together with improved data and modeling of the effects of the asteroid belt [321, 220]. Since the bound is actually on variations of $G M_{\odot}$, any future improvements in $\dot{G} / G$ beyond a part in $10^{13}$ will have to take into account models of the actual mass loss from the Sun, due to radiation of photons and neutrinos $\left(\sim 0.7 \times 10^{-13} \mathrm{yr}^{-1}\right)$ and due to the solar wind $\left(\sim 0.2 \times 10^{-13} \mathrm{yr}^{-1}\right)$. Another bound comes from LLR measurements ([436]; for earlier results see $[132,435,295])$.

Although bounds on $\dot{G} / G$ from solar-system measurements can be correctly obtained in a phenomenological manner through the simple expedient of replacing $G$ by $G_{0}+\dot{G}_{0}\left(t-t_{0}\right)$ in Newton's equations of motion, the same does not hold true for pulsar and binary pulsar timing measurements. The reason is that, in theories of gravity that violate SEP, such as scalar-tensor theories, the "mass" and moment of inertia of a gravitationally bound body may vary with $G$. Because neutron stars are highly relativistic, the fractional variation in these quantities can be comparable to $\Delta G / G$, the precise variation depending both on the equation of state of neutron star matter and on the theory of gravity in the strong-field regime. The variation in the moment of inertia affects the spin rate of the pulsar, while the variation in the mass can affect the orbital period in a manner that can subtract from the direct effect of a variation in $G$, given by $\dot{P}_{\mathrm{b}} / P_{\mathrm{b}}=$ $-2 \dot{G} / G[308]$. Thus, the bounds quoted in Table 5 for binary and millisecond pulsars are theorydependent and must be treated as merely suggestive.

In a similar manner, bounds from helioseismology and Big-Bang nucleosynthesis (BBN) assume a model for the evolution of $G$ over the multi-billion year time spans involved. For example, the concordance of predictions for light elements produced around 3 minutes after the Big Bang with the abundances observed indicate that $G$ then was within 20 percent of $G$ today. Assuming a power-law variation of $G \sim t^{-\alpha}$ then yields a bound on $\dot{G} / G$ today shown in Table 5 . 


\subsection{Other tests of post-Newtonian gravity}

\subsubsection{Search for gravitomagnetism}

According to GR, moving or rotating matter should produce a contribution to the gravitational field that is the analogue of the magnetic field of a moving charge or a magnetic dipole. In particular, one can view the $g_{0 i}$ part of the PPN metric (see Box 2) as an analogue of the vector potential of electrodynamics. In a suitable gauge (not the standard PPN gauge), and dropping the preferred-frame terms, it can be written

$$
g_{0 i}=-\frac{1}{2}\left(4 \gamma+4+\alpha_{1}\right) V_{i} .
$$

At PN order, this contributes a Lorentz-type acceleration $\mathbf{v} \times \mathbf{B}_{\mathrm{g}}$ to the equation of motion, where the gravitomagnetic field $\mathbf{B}_{\mathrm{g}}$ is given by $\mathbf{B}_{\mathrm{g}}=\nabla \times\left(g_{0 i} \mathbf{e}^{i}\right)$.

Gravitomagnetism plays a role in a variety of measured relativistic effects involving moving material sources, such as the Earth-Moon system and binary pulsar systems. Nordtvedt [307, 306] has argued that, if the gravitomagnetic potential (70) were turned off, then there would be anomalous orbital effects in LLR and binary pulsar data.

Rotation also produces a gravitomagnetic effect, since for a rotating body, $\mathbf{V}=-\frac{1}{2} \mathbf{x} \times \mathbf{J} / r^{3}$, where $\mathbf{J}$ is the angular momentum of the body. The result is a "dragging of inertial frames" around the body, also called the Lense-Thirring effect. A consequence is a precession of a gyroscope's spin $\mathbf{S}$ according to

$$
\frac{d \mathbf{S}}{d \tau}=\boldsymbol{\Omega}_{\mathrm{LT}} \times \mathbf{S}, \quad \boldsymbol{\Omega}_{\mathrm{LT}}=-\frac{1}{2}\left(1+\gamma+\frac{1}{4} \alpha_{1}\right) \frac{\mathbf{J}-3 \mathbf{n}(\mathbf{n} \cdot \mathbf{J})}{r^{3}},
$$

where $\mathbf{n}$ is a unit radial vector, and $r$ is the distance from the center of the body (TEGP 9.1 [420]).

In 2011 the Relativity Gyroscope Experiment (Gravity Probe B or GPB) carried out by Stanford University, NASA and Lockheed Martin Corporation [177], finally completed a space mission to detect this frame-dragging or Lense-Thirring precession, along with the "geodetic" precession (see Section 4.4.2). Gravity Probe B will very likely go down in the history of science as one of the most ambitious, difficult, expensive, and controversial relativity experiments ever performed. ${ }^{2}$ It was almost 50 years from inception to completion, although only about half of that time was spent as a full-fledged, approved space program.

The GPB spacecraft was launched on April 20, 2004 into an almost perfectly circular polar orbit at an altitude of $642 \mathrm{~km}$, with the orbital plane parallel to the direction of a guide star known as IM Pegasi (HR 8703). The spacecraft contained four spheres made of fuzed quartz, all spinning about the same axis (two were spun in the opposite direction), which was oriented to be in the orbital plane, pointing toward the guide star. An onboard telescope pointed continuously at the guide star, and the direction of each spin was compared with the direction to the star, which was at a declination of $16^{\circ}$ relative to the Earth's equatorial plane. With these conditions, the precessions predicted by GR were 6630 milliarcsecond per year for the geodetic effect, and 38 milliarcsecond per year for frame dragging, the former in the orbital plane (in the north-south direction) and the latter perpendicular to it (in the east-west direction).

In order to reduce the non-relativistic torques on the rotors to an acceptable level, the rotors were fabricated to be both spherical and homogenous to better than a few parts in 10 million. Each rotor was coated with a thin film of niobium, and the experiment was conducted at cryogenic temperatures inside a dewar containing 2200 litres of superfluid liquid helium. As the niobium film becomes a superconductor, each rotor develops a magnetic moment parallel to its spin axis.

${ }^{2}$ Full disclosure: The author served as Chair of an external NASA Science Advisory Committee for Gravity Probe B from 1998 to 2011. 
Variations in the direction of the magnetic moment relative to the spacecraft were then measured using superconducting current loops surrounding each rotor. As the spacecraft orbits the Earth, the aberration of light from the guide star causes an artificial but predictable change in direction between the rotors and the on-board telescope; this was an essential tool for calibrating the conversion between the voltages read by the current loops and the actual angle between the rotors and the guide star. The motion of the guide star relative to distant inertial frames was measured before, during and after the mission separately by radio astronomers at Harvard/SAO and elsewhere using VLBI (IM Pegasi is a radio star) [362].

The mission ended in September 2005, as scheduled, when the last of the liquid helium boiled off. Although all subsystems of the spacecraft and the apparatus performed extremely well, they were not perfect. Calibration measurements carried out during the mission, both before and after the science phase, revealed unexpectedly large torques on the rotors. Numerous diagnostic tests worthy of a detective novel showed that these were caused by electrostatic interactions between surface imperfections ("patch effect") on the niobium films and the spherical housings surrounding each rotor. These effects and other anomalies greatly contaminated the data and complicated its analysis, but finally, in October 2010, the Gravity Probe B team announced that the experiment had successfully measured both the geodetic and frame-dragging precessions. The outcome was in agreement with general relativity, with a precision of 0.3 percent for the geodetic precession, and 20 percent for the frame-dragging effect [149]. For a commentary on the GPB result, see [429]. The full technical and data analysis details of GPB are expected to be published as a special issue of Classical and Quantum Gravity in 2015.

Another way to look for frame-dragging is to measure the precession of orbital planes of bodies circling a rotating body. One implementation of this idea is to measure the relative precession, at about 31 milliarcseconds per year, of the line of nodes of a pair of laser-ranged geodynamics satellites (LAGEOS), ideally with supplementary inclination angles; the inclinations must be supplementary in order to cancel the dominant (126 degrees per year) nodal precession caused by the Earth's Newtonian gravitational multipole moments. Unfortunately, the two existing LAGEOS satellites are not in appropriately inclined orbits. Nevertheless, Ciufolini and collaborators $[86,88,85]$ combined nodal precession data from LAGEOS I and II with improved models for the Earth's multipole moments provided by two orbiting geodesy satellites, Europe's CHAMP (Challenging Minisatellite Payload) and NASA's GRACE (Gravity Recovery and Climate Experiment), and reported a 10 percent confirmation of GR [85]. In earlier reports, Ciufolini et al. had reported tests at the the 20-30 percent level, without the benefit of the GRACE/CHAMP data [83, 87, 82]. Some authors stressed the importance of adequately assessing systematic errors in the LAGEOS data [338, 197].

On February 13, 2012, a third laser-ranged satellite, known as LARES (Laser Relativity Satellite) was launched by the Italian Space Agency [315]. Its inclination was very close to the required supplementary angle relative to LAGEOS I, and its eccentricity was very nearly zero. However, because its semimajor axis is only $2 / 3$ that of either LAGEOS I or II, and because the Newtonian precession rate is proportional to $a^{-3 / 2}$, LARES does not provide a cancellation of the Newtonian precession. Nevertheless, combining data from all three satellites with continually improving Earth data from GRACE, the LARES team hopes to achieve a test of frame-dragging at the one percent level [84].

\subsubsection{Geodetic precession}

A gyroscope moving through curved spacetime suffers a precession of its spin axis given by

$$
\frac{d \mathbf{S}}{d \tau}=\boldsymbol{\Omega}_{\mathrm{G}} \times \mathbf{S}, \quad \boldsymbol{\Omega}_{\mathrm{G}}=\left(\gamma+\frac{1}{2}\right) \mathbf{v} \times \nabla U
$$


where $\mathbf{v}$ is the velocity of the gyroscope, and $U$ is the Newtonian gravitational potential of the source (TEGP 9.1 [420]). The Earth-Moon system can be considered as a "gyroscope", with its axis perpendicular to the orbital plane. The predicted precession is about 2 arcseconds per century, an effect first calculated by de Sitter. This effect has been measured to about 0.6 percent using LLR data [132, 435, 436].

For the GPB gyroscopes orbiting the Earth, the precession is 6.63 arcseconds per year. GPB measured this effect to $3 \times 10^{-3}$; the resulting bound on the parameter $\gamma$ is not competitive with the Cassini bound.

\subsubsection{Tests of post-Newtonian conservation laws}

Of the five "conservation law" PPN parameters $\zeta_{1}, \zeta_{2}, \zeta_{3}, \zeta_{4}$, and $\alpha_{3}$, only three, $\zeta_{2}, \zeta_{3}$, and $\alpha_{3}$, have been constrained directly with any precision; $\zeta_{1}$ is constrained indirectly through its appearance in the Nordtvedt effect parameter $\eta_{\mathrm{N}}$, Eq. (68). There is strong theoretical evidence that $\zeta_{4}$, which is related to the gravity generated by fluid pressure, is not really an independent parameter - in any reasonable theory of gravity there should be a connection between the gravity produced by kinetic energy $\left(\rho v^{2}\right)$, internal energy $(\rho \Pi)$, and pressure $(p)$. From such considerations, there follows [414] the additional theoretical constraint

$$
6 \zeta_{4}=3 \alpha_{3}+2 \zeta_{1}-3 \zeta_{3} .
$$

A non-zero value for any of these parameters would result in a violation of conservation of momentum, or of Newton's third law in gravitating systems. An alternative statement of Newton's third law for gravitating systems is that the "active gravitational mass", that is the mass that determines the gravitational potential exhibited by a body, should equal the "passive gravitational mass", the mass that determines the force on a body in a gravitational field. Such an equality guarantees the equality of action and reaction and of conservation of momentum, at least in the Newtonian limit.

A classic test of Newton's third law for gravitating systems was carried out in 1968 by Kreuzer, in which the gravitational attraction of fluorine and bromine were compared to a precision of 5 parts in $10^{5}$.

A remarkable planetary test was reported by Bartlett and van Buren [33]. They noted that current understanding of the structure of the Moon involves an iron-rich, aluminum-poor mantle whose center of mass is offset about $10 \mathrm{~km}$ from the center of mass of an aluminum-rich, ironpoor crust. The direction of offset is toward the Earth, about $14^{\circ}$ to the east of the Earth-Moon line. Such a model accounts for the basaltic maria which face the Earth, and the aluminum-rich highlands on the Moon's far side, and for a $2 \mathrm{~km}$ offset between the observed center of mass and center of figure for the Moon. Because of this asymmetry, a violation of Newton's third law for aluminum and iron would result in a momentum non-conserving self-force on the Moon, whose component along the orbital direction would contribute to the secular acceleration of the lunar orbit. Improved knowledge of the lunar orbit through LLR, and a better understanding of tidal effects in the Earth-Moon system (which also contribute to the secular acceleration) through satellite data, severely limit any anomalous secular acceleration, with the resulting limit

$$
\left|\frac{\left(m_{\mathrm{A}} / m_{\mathrm{P}}\right)_{\mathrm{Al}}-\left(m_{\mathrm{A}} / m_{\mathrm{P}}\right)_{\mathrm{Fe}}}{\left(m_{\mathrm{A}} / m_{\mathrm{P}}\right)_{\mathrm{Fe}}}\right|<4 \times 10^{-12} .
$$

According to the PPN formalism, in a theory of gravity that violates conservation of momentum, but that obeys the constraint of Eq. (73), the electrostatic binding energy $E_{\mathrm{e}}$ of an atomic nucleus could make a contribution to the ratio of active to passive mass of the form

$$
m_{\mathrm{A}}=m_{\mathrm{P}}+\frac{1}{2} \zeta_{3} E_{\mathrm{e}} .
$$


The resulting limit on $\zeta_{3}$ from the lunar experiment is $\zeta_{3}<1 \times 10^{-8}$ (TEGP 9.2, 14.3 (d) [420]). Nordtvedt [310] has examined whether this bound could be improved by considering the asymmetric distribution of ocean water on Earth.

Another consequence of a violation of conservation of momentum is a self-acceleration of the center of mass of a binary stellar system, given by

$$
\mathbf{a}_{\mathrm{CM}}=-\frac{1}{2}\left(\zeta_{2}+\alpha_{3}\right) \frac{m}{a^{2}} \frac{\mu}{a} \frac{\delta m}{m} \frac{e}{\left(1-e^{2}\right)^{3 / 2}} \mathbf{n}_{\mathrm{P}},
$$

where $\delta m=m_{1}-m_{2}, a$ is the semi-major axis, and $\mathbf{n}_{\mathrm{P}}$ is a unit vector directed from the center of mass to the point of periastron of $m_{1}$ (TEGP 9.3 [420]). A consequence of this acceleration would be non-vanishing values for $d^{2} P / d t^{2}$, where $P$ denotes the period of any intrinsic process in the system (orbit, spectra, pulsar periods). The observed upper limit on $d^{2} P_{\mathrm{p}} / d t^{2}$ of the binary pulsar PSR 1913+16 places a strong constraint on such an effect, resulting in the bound $\left|\alpha_{3}+\zeta_{2}\right|<4 \times 10^{-5}$. Since $\alpha_{3}$ has already been constrained to be much less than this (see Table 4), we obtain a strong solitary bound on $\zeta_{2}<4 \times 10^{-5}$ [419].

\subsection{Prospects for improved PPN parameter values}

A number of advanced experiments or space missions are under development or have been proposed which could lead to significant improvements in values of the PPN parameters, of $J_{2}$ of the Sun, and of $\dot{G} / G$.

LLR at the Apache Point Observatory (APOLLO project) could improve bounds on the Nordvedt parameter to the level $3 \times 10^{-5}$ and on $\dot{G} / G$ to better than $10^{-13} \mathrm{yr}^{-1}$ [437].

The BepiColumbo Mercury orbiter is a joint project of the European and Japanese space agencies, scheduled for launch in 2015 [38]. In a two-year experiment, with $6 \mathrm{~cm}$ range capability, it could yield improvements in $\gamma$ to $3 \times 10^{-5}$, in $\beta$ to $3 \times 10^{-4}$, in $\alpha_{1}$ to $10^{-5}$, in $\dot{G} / G$ to $10^{-13} \mathrm{yr}^{-1}$, and in $J_{2}$ to $3 \times 10^{-8}$. An eight-year mission could yield further improvements by factors of $2-5$ in $\beta, \alpha_{1}$, and $J_{2}$, and a further factor 15 in $\dot{G} / G$ [282,27].

GAIA is a high-precision astrometric orbiting telescope launched by ESA in 2013 (a successor to Hipparcos) [169]. With astrometric capability ranging from 10 to a few hundred microsarcseconds, plus the ability measure the locations of a billion stars down to 20th magnitude, it could measure light-deflection and $\gamma$ to the $10^{-6}$ level [281].

LATOR (Laser Astrometric Test of Relativity) is a concept for a NASA mission in which two microsatellites orbit the Sun on Earth-like orbits near superior conjunction, so that their lines of sight are close to the Sun. Using optical tracking and an optical interferometer on the International Space Station, it may be possible to measure the deflection of light with sufficient accuracy to bound $\gamma$ to a part in $10^{8}$ and $J_{2}$ to a part in $10^{8}$, and to measure the solar frame-dragging effect to one percent [393, 394].

Another concept, proposed for a European Space Agency medium-class mission, is ASTROD I (Astrodynamical Space Test of Relativity using Optical Devices), a variant of LATOR involving a single satellite parked on the far side of the Sun [66]. Its goal is to measure $\gamma$ to a few parts in $10^{8}, \beta$ to six parts in $10^{6}$ and $J_{2}$ to a part in $10^{9}$. A possible follow-on mission, ASTROD-GW, involving three spacecraft, would improve on measurements of those parameters and would also measure the solar frame-dragging effect, as well as look for gravitational waves. 


\section{Strong Gravity and Gravitational Waves: Tests for the 21st Century}

\subsection{Strong-field systems in general relativity}

\subsubsection{Defining weak and strong gravity}

In the solar system, gravity is weak, in the sense that the Newtonian gravitational potential and related variables $\left(U(\mathbf{x}, t) \sim v^{2} \sim p / \rho \sim \epsilon\right)$ are much smaller than unity everywhere. This is the basis for the post-Newtonian expansion and for the "parametrized post-Newtonian" framework described in Section 3.2. "Strong-field" systems are those for which the simple 1PN approximation of the PPN framework is no longer appropriate. This can occur in a number of situations:

- The system may contain strongly relativistic objects, such as neutron stars or black holes, near and inside which $\epsilon \sim 1$, and the post-Newtonian approximation breaks down. Nevertheless, under some circumstances, the orbital motion may be such that the interbody potential and orbital velocities still satisfy $\epsilon \ll 1$ so that a kind of post-Newtonian approximation for the orbital motion might work; however, the strong-field internal gravity of the bodies could (especially in alternative theories of gravity) leave imprints on the orbital motion.

- The evolution of the system may be affected by the emission of gravitational radiation. The 1PN approximation does not contain the effects of gravitational radiation back-reaction. In the expression for the metric given in Box 2, radiation back-reaction effects in GR do not occur until $\mathcal{O}\left(\epsilon^{7 / 2}\right)$ in $g_{00}, \mathcal{O}\left(\epsilon^{3}\right)$ in $g_{0 i}$, and $\mathcal{O}\left(\epsilon^{5 / 2}\right)$ in $g_{i j}$. Consequently, in order to describe such systems, one must carry out a solution of the equations substantially beyond 1PN order, sufficient to incorporate the leading radiation damping terms at $2.5 \mathrm{PN}$ order. In addition, the PPN metric described in Section 3.2 is valid in the near zone of the system, i.e., within one gravitational wavelength of the system's center of mass. As such it cannot describe the gravitational waves seen by a detector.

- The system may be highly relativistic in its orbital motion, so that $U \sim v^{2} \sim 1$ even for the interbody field and orbital velocity. Systems like this include the late stage of the inspiral of binary systems of neutron stars or black holes, driven by gravitational radiation damping, prior to a merger and collapse to a final stationary state. Binary inspiral is one of the leading candidate sources for detection by the existing LIGO-Virgo network of laser interferometric gravitational-wave observatories and by a future space-based interferometer. A proper description of such systems requires not only equations for the motion of the binary carried to extraordinarily high $\mathrm{PN}$ orders (at least 3.5PN), but also requires equations for the far-zone gravitational waveform measured at the detector, that are equally accurate to high PN orders beyond the leading "quadrupole" approximation.

Of course, some systems cannot be properly described by any post-Newtonian approximation because their behavior is fundamentally controlled by strong gravity. These include the imploding cores of supernovae, the final merger of two compact objects, the quasinormal-mode vibrations of neutron stars and black holes, the structure of rapidly rotating neutron stars, and so on. Phenomena such as these must be analyzed using different techniques. Chief among these is the full solution of Einstein's equations via numerical methods. This field of "numerical relativity" has become a mature branch of gravitational physics, whose description is beyond the scope of this review (see [247, 176, 35] for reviews). Another is black-hole perturbation theory (see [285, 219, 351, 43] for reviews). 


\subsubsection{Compact bodies and the strong equivalence principle}

When dealing with the motion and gravitational-wave generation by orbiting bodies, one finds a remarkable simplification within GR. As long as the bodies are sufficiently well-separated that one can ignore tidal interactions and other effects that depend upon the finite extent of the bodies (such as their quadrupole and higher multipole moments), then all aspects of their orbital behavior and gravitational-wave generation can be characterized by just two parameters: mass and angular momentum. Whether their internal structure is highly relativistic, as in black holes or neutron stars, or non-relativistic as in the Earth and Sun, only the mass and angular momentum are needed. Furthermore, both quantities are measurable in principle by examining the external gravitational field of the bodies, and make no reference whatsoever to their interiors.

Damour [100] calls this the "effacement" of the bodies' internal structure. It is a consequence of the strong equivalence principle (SEP), described in Section 3.1.2.

General relativity satisfies SEP because it contains one and only one gravitational field, the spacetime metric $g_{\mu \nu}$. Consider the motion of a body in a binary system, whose size is small compared to the binary separation. Surround the body by a region that is large compared to the size of the body, yet small compared to the separation. Because of the general covariance of the theory, one can choose a freely-falling coordinate system which comoves with the body, whose spacetime metric takes the Minkowski form at its outer boundary (ignoring tidal effects generated by the companion). There is thus no evidence of the presence of the companion body, and the structure of the chosen body can be obtained using the field equations of GR in this coordinate system. Far from the chosen body, the metric is characterized by the mass and angular momentum (assuming that one ignores quadrupole and higher multipole moments of the body) as measured far from the body using orbiting test particles and gyroscopes. These asymptotically measured quantities are oblivious to the body's internal structure. A black hole of mass $m$ and a planet of mass $m$ would produce identical spacetimes in this outer region.

The geometry of this region surrounding the one body must be matched to the geometry provided by the companion body. Einstein's equations provide consistency conditions for this matching that yield constraints on the motion of the bodies. These are the equations of motion. As a result, the motion of two planets of mass and angular momentum $m_{1}, m_{2}, \mathbf{J}_{1}$, and $\mathbf{J}_{2}$ is identical to that of two black holes of the same mass and angular momentum (again, ignoring tidal effects).

This effacement does not occur in an alternative gravitional theory like scalar-tensor gravity. There, in addition to the spacetime metric, a scalar field $\phi$ is generated by the masses of the bodies, and controls the local value of the gravitational coupling constant (i.e., $G_{\text {Local }}$ is a function of $\phi$ ). Now, in the local frame surrounding one of the bodies in our binary system, while the metric can still be made Minkowskian far away, the scalar field will take on a value $\phi_{0}$ determined by the companion body. This can affect the value of $G_{\text {Local }}$ inside the chosen body, alter its internal structure (specifically its gravitational binding energy) and hence alter its mass. Effectively, each body can be characterized by several mass functions $m_{A}(\phi)$, which depend on the value of the scalar field at its location, and several distinct masses come into play, such as inertial mass, gravitational mass, "radiation" mass, etc. The precise nature of the functions will depend on the body, specifically on its gravitational binding energy, and as a result, the motion and gravitational radiation may depend on the internal structure of each body. For compact bodies such as neutron stars and black holes these internal structure effects could be large; for example, the gravitational binding energy of a neutron star can be 10-20 percent of its total mass. At 1PN order, the leading manifestation of this phenomenon is the Nordtvedt effect.

This is how the study of orbiting systems containing compact objects provides strong-field tests of GR. Even though the strong-field nature of the bodies is effaced in GR, it is not in other theories, thus any result in agreement with the predictions of GR constitutes a kind of "null" test of strong-field gravity. 


\subsection{Motion and gravitational radiation in general relativity: A history}

At the most primitive level, the problem of motion in GR is relatively straightforward, and was an integral part of the theory as proposed by Einstein ${ }^{3}$. The first attempts to treat the motion of multiple bodies, each with a finite mass, were made in the period 1916-1917 by Lorentz and Droste and by de Sitter $[260,124]$. They derived the metric and equations of motion for a system of $N$ bodies, in what today would be called the first post-Newtonian approximation of GR (de Sitter's equations turned out to contain some important errors). In 1916, Einstein took the first crack at a study of gravitational radiation, deriving the energy emitted by a body such as a rotating rod or dumbbell, held together by non-gravitational forces [143, 144]. He made some unjustified assumptions as well as a trivial numerical error (later corrected by Eddington [141]), but the underlying conclusion that dynamical systems would radiate gravitational waves was correct.

The next significant advance in the problem of motion came 20 years later. In 1938, Einstein, Infeld and Hoffman published the now legendary "EIH" paper, a calculation of the $N$-body equations of motion using only the vacuum field equations of GR [145]. They treated each body in the system as a spherically symmetric object whose nearby vacuum exterior geometry approximated that of the Schwarzschild metric of a static spherical star. They then solved the vacuum field equations for the metric between each body in the system in a weak field, slow-motion approximation. Then, using a primitive version of what today would be called "matched asymptotic expansions" they showed that, in order for the nearby metric of each body to match smoothly to the interbody metric at each order in the expansion, certain conditions on the motion of each body had to be met. Together, these conditions turned out to be equivalent to the Droste-Lorentz $N$-body equations of motion. The internal structure of each body was irrelevant, apart from the requirement that its nearby field be approximately spherically symmetric, a clear illustration of the "effacement" principle.

Around the same time, there occurred an unusual detour in the problem of motion. Using equations of motion based on de Sitter's paper, specialized to two bodies, Levi-Civita [249] showed that the center of mass of a binary star system would suffer an acceleration in the direction of the pericenter of the orbit, in an amount proportional to the difference between the two masses, and to the eccentricity of the orbit. Such an effect would be a violation of the conservation of momentum for isolated systems caused by relativistic gravitational effects. Levi-Civita even went so far as to suggest looking for this effect in selected nearby close binary star systems. However, Eddington and Clark [140] quickly pointed out that Levi-Civita had based his calculations on de Sitter's flawed work; when correct two-body equations of motion were used, the effect vanished, and momentum conservation was upheld. Robertson confirmed this using the EIH equations of motion [341]. Such an effect can only occur in theories of gravity that lack the appropriate conservation laws (Section 4.4.3).

There was ongoing confusion over whether gravitational waves are real or are artifacts of general covariance. Although Eddington was credited with making the unfortunate remark that gravitational waves propagate "with the speed of thought", he did clearly elucidate the difference between the physical, coordinate independent modes and modes that were purely coordinate artifacts [141]. But in 1936, in a paper submitted to the Physical Review, Einstein and Rosen claimed to prove that gravitational waves could not exist; the anonymous referee of their paper found that they had made an error. Upset that the journal had sent his paper to a referee (a newly instituted practice), Einstein refused to publish there again. A corrected paper by Einstein and Rosen showing that gravitational waves did exist - cylindrical waves in this case - was published elsewhere [146]. Fifty years later it was revealed that the anonymous referee was H. P. Robertson [213].

Roughly 20 more years would pass before another major attack on the problem of motion.

${ }^{3}$ This history is adapted from Ref. [430]. For a detailed technical and historical review of the problem of motion, see Damour [100] 
Fock in the USSR and Chandrasekhar in the US independently developed and systematized the post-Newtonian approximation in a form that laid the foundation for modern post-Newtonian theory $[160,77]$. They developed a full post-Newtonian hydrodynamics, with the ability to treat realistic, self-gravitating bodies of fluid, such as stars and planets. In the suitable limit of "point" particles, or bodies whose size is small enough compared to the interbody separations that finitesize effects such as spin and tidal interactions can be ignored, their equations of motion could be shown to be equivalent to the EIH and the Droste-Lorentz equations of motion.

The next important period in the history of the problem of motion was 1974-1979, initiated by the 1974 discovery of the binary pulsar PSR $1913+16$ by Hulse and Taylor [196]. Around the same time there occurred the first serious attempt to calculate the head-on collision of two black holes using purely numerical solutions of Einstein's equations, by Smarr and collaborators [368].

The binary pulsar consists of two neutron stars, one an active pulsar detectable by radio telescopes, the other very likely an old, inactive pulsar (Section 6.1). Each neutron star has a mass of around 1.4 solar masses. The orbit of the system was seen immediately to be quite relativistic, with an orbital period of only eight hours, and a mean orbital speed of $200 \mathrm{~km} / \mathrm{s}$, some four times faster than Mercury in its orbit. Within weeks of its discovery, numerous authors pointed out that PSR 1913+16 would be an important new testing ground for GR. In particular, it could provide for the first time a test of the effects of the emission of gravitational radiation on the orbit of the system.

However, the discovery revealed an ugly truth about the "problem of motion". As Ehlers et al. pointed out in an influential 1976 paper [142], the general relativistic problem of motion and radiation was full of holes large enough to drive trucks through. They pointed out that most treatments of the problem used "delta functions" as a way to approximate the bodies in the system as point masses. As a consequence, the "self-field", the gravitational field of the body evaluated at its own location, becomes infinite. While this is not a major issue in Newtonian gravity or classical electrodynamics, the non-linear nature of GR requires that this infinite self-field contribute to gravity. In the past, such infinities had been simply swept under the rug. Similarly, because gravitational energy itself produces gravity it thus acts as a source throughout spacetime. This means that, when calculating radiative fields, integrals for the multipole moments of the source that are so useful in treating radiation begin to diverge. These divergent integrals had also been routinely swept under the rug. Ehlers et al. further pointed out that the true boundary condition for any problem involving radiation by an isolated system should be one of "no incoming radiation" from the past. Connecting this boundary condition with the routine use of retarded solutions of wave equations was not a trivial matter in GR. Finally, they pointed out that there was no evidence that the post-Newtonian approximation, so central to the problem of motion, was a convergent or even asymptotic sequence. Nor had the approximation been carried out to high enough order to make credible error estimates.

During this time, some authors even argued that the "quadrupole formula" for the gravitational energy emitted by a system (see below), while correct for a rotating dumbell as calculated by Einstein, was actually wrong for a binary system moving under its own gravity. The discovery in 1979 that the rate of decay of the orbit of the binary pulsar was in agreement with the standard quadrupole formula made some of these arguments moot. Yet the question raised by Ehlers et al. was still relevant: is the quadrupole formula for binary systems an actual prediction of GR?

Motivated by the Ehlers et al. critique, numerous workers began to address the holes in the problem of motion, and by the late 1990s most of the criticisms had been answered, particularly those related to divergences. For a detailed history of the ups and downs of the subject of motion and gravitational waves, see [214].

The problem of motion and radiation in GR has received renewed interest since 1990, with proposals for construction of large-scale laser interferometric gravitational-wave observatories. These proposals culminated in the construction and operation of LIGO in the US, VIRGO and GEO600 
in Europe, and TAMA300 in Japan, the construction of an underground observatory KAGRA in Japan, and the possible construction of a version of LIGO in India. Advanced versions of LIGO and VIRGO are expected to be online and detecting gravitational waves around 2016. An interferometer in space has recently been selected by the European Space Agency for a launch in the 2034 time frame.

A leading candidate source of detectable waves is the inspiral, driven by gravitational radiation damping, of a binary system of compact objects (neutron stars or black holes) (for a review of sources of gravitational waves, see [352]). The analysis of signals from such systems will require theoretical predictions from GR that are extremely accurate, well beyond the leading-order prediction of Newtonian or even post-Newtonian gravity for the orbits, and well beyond the leading-order formulae for gravitational waves.

This presented a major theoretical challenge: to calculate the motion and radiation of systems of compact objects to very high PN order, a formidable algebraic task, while addressing the issues of principle raised by Ehlers et al., sufficiently well to ensure that the results were physically meaningful. This challenge has been largely met, so that we may soon see a remarkable convergence between observational data and accurate predictions of gravitational theory that could provide new, strong-field tests of GR.

\subsection{Compact binary systems in general relativity}

\subsubsection{Einstein's equations in "relaxed" form}

Here we give a brief overview of the modern approach to the problem of motion and gravitational radiation in GR. For a full pedagogical treatment, see [324].

The Einstein equations $G_{\mu \nu}=8 \pi G T_{\mu \nu}$ are elegant and deceptively simple, showing geometry (in the form of the Einstein tensor $G_{\mu \nu}$, which is a function of spacetime curvature) being generated by matter (in the form of the material stress-energy tensor $T_{\mu \nu}$ ). However, this is not the most useful form for actual calculations. For post-Newtonian calculations, a far more useful form is the so-called "relaxed" Einstein equations, which form the basis of the program of approximating solutions of Einstein's equations known as post-Minkowskian theory and post-Newtonian theory. The starting point is the so-called "gothic inverse metric", defined by $\mathfrak{g}^{\alpha \beta} \equiv \sqrt{-g} g^{\alpha \beta}$, where $g$ is the determinant of $g_{\alpha \beta}$. One then defines the gravitational potential $h^{\alpha \beta} \equiv \eta^{\alpha \beta}-\mathfrak{g}^{\alpha \beta}$. After imposing the de Donder or harmonic gauge condition $\partial h^{\alpha \beta} / \partial x^{\beta}=0$ (summation on repeated indices is assumed), one can recast the exact Einstein field equations into the form

$$
\square h^{\alpha \beta}=-16 \pi G \tau^{\alpha \beta},
$$

where $\square \equiv-\partial^{2} / \partial t^{2}+\nabla^{2}$ is the flat-spacetime wave operator. This form of Einstein's equations bears a striking similarity to Maxwell's equations for the vector potential $A^{\alpha}$ in Lorentz gauge: $\square A^{\alpha}=-4 \pi J^{\alpha}, \partial A^{\alpha} / \partial x^{\alpha}=0$. There is a key difference, however: The source on the right hand side of Eq. (77) is given by the "effective" stress-energy pseudotensor

$$
\tau^{\alpha \beta}=(-g)\left(T^{\alpha \beta}+t_{\mathrm{LL}}^{\alpha \beta}+t_{\mathrm{H}}^{\alpha \beta}\right)
$$

where $t_{\mathrm{LL}}^{\alpha \beta}$ and $t_{\mathrm{H}}^{\alpha \beta}$ are the Landau-Lifshitz pseudotensor and a harmonic pseudotensor, given by terms quadratic (and higher) in $h^{\alpha \beta}$ and its derivatives (see [324], Eqs. $(6.5,6.52,6.53)$ for explicit formulae). In GR, the gravitational field itself generates gravity, a reflection of the nonlinearity of Einstein's equations, and in contrast to the linearity of Maxwell's equations.

Eq. (77) is exact, and depends only on the assumption that the relevant parts of spacetime can be covered by harmonic coordinates. It is called "relaxed" because it can be solved formally 
as a functional of source variables without specifying the motion of the source, in the form (with $G=1)$

$$
h^{\alpha \beta}(t, \mathbf{x})=4 \int_{\mathcal{C}} \frac{\tau^{\alpha \beta}\left(t-\left|\mathbf{x}-\mathbf{x}^{\prime}\right|, \mathbf{x}^{\prime}\right)}{\left|\mathbf{x}-\mathbf{x}^{\prime}\right|} d^{3} x^{\prime},
$$

where the integration is over the past flat-spacetime null cone $\mathcal{C}$ of the field point $(t, \mathbf{x})$. The motion of the source is then determined either by the equation $\partial \tau^{\alpha \beta} / \partial x^{\beta}=0$ (which follows from the harmonic gauge condition), or from the usual covariant equation of motion $T^{\alpha \beta}{ }_{; \beta}=0$, where the subscript $; \beta$ denotes a covariant divergence. This formal solution can then be iterated in a slow motion $(v<1)$ weak-field $\left(\left\|h^{\alpha \beta}\right\|<1\right)$ approximation. One begins by substituting $h_{0}^{\alpha \beta}=0$ into the source $\tau^{\alpha \beta}$ in Eq. (79), and solving for the first iterate $h_{1}^{\alpha \beta}$, and then repeating the procedure sufficiently many times to achieve a solution of the desired accuracy. For example, to obtain the $1 \mathrm{PN}$ equations of motion, two iterations are needed (i.e., $h_{2}^{\alpha \beta}$ must be calculated); likewise, to obtain the leading gravitational waveform for a binary system, two iterations are needed.

At the same time, just as in electromagnetism, the formal integral (79) must be handled differently, depending on whether the field point is in the far zone or the near zone. For field points in the far zone or radiation zone, $|\mathbf{x}|>\mathcal{R}$, where $\mathcal{R}$ is a distance of the order of a gravitational wavelength, the field can be expanded in inverse powers of $R=|\mathbf{x}|$ in a multipole expansion, evaluated at the "retarded time" $t-R$. The leading term in $1 / R$ is the gravitational waveform. For field points in the near zone or induction zone, $|\mathbf{x}| \sim\left|\mathbf{x}^{\prime}\right|<\mathcal{R}$, the field is expanded in powers of $\left|\mathbf{x}-\mathbf{x}^{\prime}\right|$ about the local time $t$, yielding instantaneous potentials that go into the equations of motion.

However, because the source $\tau^{\alpha \beta}$ contains $h^{\alpha \beta}$ itself, it is not confined to a compact region, but extends over all spacetime. As a result, there is a danger that the integrals involved in the various expansions will diverge or be ill-defined. This consequence of the non-linearity of Einstein's equations has bedeviled the subject of gravitational radiation for decades. Numerous approaches have been developed to try to handle this difficulty. The post-Minkowskian method of Blanchet, Damour, and Iyer $[52,53,54,108,55,50]$ solves Einstein's equations by two different techniques, one in the near zone and one in the far zone, and uses the method of singular asymptotic matching to join the solutions in an overlap region. The method provides a natural "regularization" technique to control potentially divergent integrals (see [51] for a thorough review). The "Direct Integration of the Relaxed Einstein Equations" (DIRE) approach of Will, Wiseman, and Pati [432, 316, 317] retains Eq. (79) as the global solution, but splits the integration into one over the near zone and another over the far zone, and uses different integration variables to carry out the explicit integrals over the two zones. In the DIRE method, all integrals are finite and convergent. Itoh and Futamase used an extension of the Einstein-Infeld-Hoffman matching approach combined with a specific method for taking a point-particle limit [198], while Damour, Jaranowski, and Schäfer pioneered an ADM Hamiltonian approach that focuses on the equations of motion [206, 207, 109, 110, 111].

These methods assume from the outset that gravity is sufficiently weak that $\left\|h^{\alpha \beta}\right\|<1$ and harmonic coordinates exists everywhere, including inside the bodies. Thus, in order to apply the results to cases where the bodies may be neutron stars or black holes, one relies upon the SEP to argue that, if tidal forces are ignored, and equations are expressed in terms of masses and spins, one can simply extrapolate the results unchanged to the situation where the bodies are ultrarelativistic. While no general proof of this exists, it has been shown to be valid in specific circumstances, such as through $2 \mathrm{PN}$ order in the equations of motion $[178,290]$, and for black holes moving in a Newtonian background field [100].

Methods such as these have resolved most of the issues that led to criticism of the foundations of gravitational radiation theory during the 1970s. 


\subsubsection{Equations of motion and gravitational waveform}

Among the results of these approaches are formulae for the equations of motion and gravitational waveform of binary systems of compact objects, carried out to high orders in a PN expansion. For a review of the latest results of high-order PN calculations, see [51]. Here we shall only state the key formulae that will be needed for this review. For example, the relative two-body equation of motion has the form

$$
\mathbf{a}=\frac{d \mathbf{v}}{d t}=\frac{m}{r^{2}}\left\{-\hat{\mathbf{n}}+\mathbf{A}_{1 \mathrm{PN}}+\mathbf{A}_{2 \mathrm{PN}}+\mathbf{A}_{2.5 \mathrm{PN}}+\mathbf{A}_{3 \mathrm{PN}}+\mathbf{A}_{3.5 \mathrm{PN}}+\ldots\right\},
$$

where $m=m_{1}+m_{2}$ is the total mass, $r=\left|\mathbf{x}_{1}-\mathbf{x}_{2}\right|, \mathbf{v}=\mathbf{v}_{1}-\mathbf{v}_{2}$, and $\hat{\mathbf{n}}=\left(\mathbf{x}_{1}-\mathbf{x}_{2}\right) / r$. The notation $\mathbf{A}_{n \text { PN }}$ indicates that the term is $\mathcal{O}\left(\epsilon^{n}\right)$ relative to the Newtonian term $-\hat{\mathbf{n}}$ (recall that $\epsilon \sim v^{2} \sim m / r$ ). Explicit and unambiguous formulae for non-spinning bodies through 3.5PN order have been calculated by various authors [51]. Here we quote only the 1PN corrections and the leading radiation-reaction terms at $2.5 \mathrm{PN}$ order:

$$
\begin{aligned}
\mathbf{A}_{1 \mathrm{PN}} & =\left\{(4+2 \eta) \frac{m}{r}-(1+3 \eta) v^{2}+\frac{3}{2} \eta \dot{r}^{2}\right\} \hat{\mathbf{n}}+(4-2 \eta) \dot{r} \mathbf{v}, \\
\mathbf{A}_{2.5 \mathrm{PN}} & =\frac{8}{5} \eta \frac{m}{r}\left\{\left(3 v^{2}+\frac{17}{3} \frac{m}{r}\right) \dot{r} \hat{\mathbf{n}}-\left(v^{2}+3 \frac{m}{r}\right) \mathbf{v}\right\},
\end{aligned}
$$

where $\eta=m_{1} m_{2} /\left(m_{1}+m_{2}\right)^{2}$. The radiation-reaction acceleration is expressed in the so-called Damour-Deruelle gauge. These terms are sufficient to analyze the orbit and evolution of the binary pulsar (see Section 6.1). For example, the 1PN terms are responsible for the periastron advance of an eccentric orbit, given by

$$
\dot{\omega}=\frac{6 \pi m f_{b}}{a\left(1-e^{2}\right)} .
$$

where $a$ and $e$ are the semi-major axis and eccentricity of the orbit, respectively, and $f_{\mathrm{b}}$ is the orbital frequency, given to the needed order by Kepler's third law $2 \pi f_{\mathrm{b}}=\left(\mathrm{m} / \mathrm{a}^{3}\right)^{1 / 2}$.

Another product is a formula for the gravitational field far from the system, written schematically in the form

$$
h^{i j}=\frac{2 m}{R}\left\{Q^{i j}+Q_{0.5 \mathrm{PN}}^{i j}+Q_{1 \mathrm{PN}}^{i j}+Q_{1.5 \mathrm{PN}}^{i j}+Q_{2 \mathrm{PN}}^{i j}+Q_{2.5 \mathrm{PN}}^{i j}+\ldots\right\},
$$

where $R$ is the distance from the source, and the variables are to be evaluated at retarded time $t-R$. The leading term is the so-called quadrupole formula

$$
h^{i j}(t, \mathbf{x})=\frac{2}{R} \ddot{I}^{i j}(t-R),
$$

where $I^{i j}$ is the quadrupole moment of the source, and overdots denote time derivatives. For a binary system this leads to

$$
Q^{i j}=2 \eta\left(v^{i} v^{j}-\frac{m \hat{n}^{i} \hat{n}^{j}}{r}\right) .
$$

For binary systems, explicit formulae for the waveform through 3.5PN order have been derived (see [56] for a ready-to-use presentation of the waveform to 2PN order for circular orbits; see [51] for a full review).

Given the gravitational waveform, one can compute the rate at which energy is carried off by the radiation (schematically $\int \dot{h} \dot{h} d \Omega$, the gravitational analog of the Poynting flux). The lowest-order quadrupole formula leads to the gravitational wave energy flux

$$
\dot{E}=\frac{8}{15} \frac{\mu \eta}{r}\left(\frac{m}{r}\right)^{3}\left(12 v^{2}-11 \dot{r}^{2}\right) .
$$


This has been extended to 3.5PN order beyond the quadrupole formula [51]. Formulae for fluxes of angular and linear momentum can also be derived. The $2.5 \mathrm{PN}$ radiation-reaction terms in the equation of motion (80) result in a damping of the orbital energy that precisely balances the energy flux (87) determined from the waveform. Averaged over one orbit, this results in a rate of increase of the binary's orbital frequency given by

$$
\begin{aligned}
\dot{f}_{\mathrm{b}} & =\frac{192 \pi}{5} f_{\mathrm{b}}^{2}\left(2 \pi \mathcal{M} f_{\mathrm{b}}\right)^{5 / 3} F(e), \\
F(e) & =\left(1-e^{2}\right)^{-7 / 2}\left(1+\frac{73}{24} e^{2}+\frac{37}{96} e^{4}\right),
\end{aligned}
$$

where $\mathcal{M}$ is the so-called "chirp" mass, given by $\mathcal{M}=\eta^{3 / 5} m$. Notice that by making precise measurements of the phase $\Phi(t)=2 \pi \int^{t} f\left(t^{\prime}\right) d t^{\prime}$ of either the orbit or the gravitational waves (for which $f=2 f_{\mathrm{b}}$ for the dominant component) as a function of the frequency, one in effect measures the "chirp" mass of the system.

These formalisms have also been generalized to include the leading effects of spin-orbit and spin-spin coupling between the bodies as well as many next-to-leading-order corrections [51].

Another approach to gravitational radiation is applicable to the special limit in which one mass is much smaller than the other. This is the method of black hole perturbation theory. One begins with an exact background spacetime of a black hole, either the non-rotating Schwarzschild or the rotating Kerr solution, and perturbs it according to $g_{\mu \nu}=g_{\mu \nu}^{(0)}+h_{\mu \nu}$. The particle moves on a geodesic of the background spacetime, and a suitably defined source stress-energy tensor for the particle acts as a source for the gravitational perturbation and wave field $h_{\mu \nu}$. This method provides numerical results that are exact in $v$, as well as analytical results expressed as series in powers of $v$, both for non-rotating and for rotating black holes. For non-rotating holes, the analytical expansions have been carried to the impressive level of $22 \mathrm{PN}$ order, or $\epsilon^{22}$ beyond the quadrupole approximation [168], and for rotating Kerr black holes, to 20PN order [356]. All results of black hole perturbation agree precisely with the $m_{1} \rightarrow 0$ limit of the PN results, up to the highest PN order where they can be compared (for reviews of earlier work see [285, 219, 351]).

\subsection{Compact binary systems in scalar-tensor theories}

Because of the recent resurgence of interest in scalar-tensor theories of gravity, motivated in part by string theory and $f(R)$ theories, considerable work has been done to analyze the motion and gravitational radiation from systems of compact objects in this class of theories. In earlier work, Eardley [139] was the first to point out the existence of dipole gravitational radiation from selfgravitating bodies in Brans-Dicke theory, and Will [415] worked out the lowest-order monopole, dipole and quadrupole radiation flux in general scalar-tensor theories (as well as in a number of alternative theories) for bodies with weak self-gravity. Using the approach pioneered by Eardley [139] for incorporating strongly self-gravitating bodies into scalar-tensor calculations, Will and Zaglauer [434] calculated the 1PN equations of motion along with the monopole-quadrupole and dipole energy flux for compact binary systems; Alsing et al. [7] extended these results to the case of Brans-Dicke theory with a massive scalar field. However, the expressions for the energy flux in those works were incomplete, because they failed to include some important post-Newtonian corrections in the scalar part of the radiation that actually contribute at the same order as the quadrupole contributions from the tensor part. Damour and Esposito-Farèse [105] obtained the correct monopole-quadrupole and dipole energy flux, working in the Einstein-frame representation of scalar-tensor theories, and gave partial results for the equations of motion to $2 \mathrm{PN}$ order. Mirshekari and Will [286] obtained the complete compact-binary equations of motion in general scalar-tensor theories through $2.5 \mathrm{PN}$ order, and obtained the energy loss rate in complete agreement with the flux result from Damour and Esposito-Farèse. Lang [241] obtained the tensor 
gravitational-wave signal to $2 \mathrm{PN}$ order.

Notwithstanding the very tight bound on the scalar-tensor coupling parameter $\omega$ from Cassini measurements in the solar system, this effort is motivated by a desire to test this theory in strongfield situations, whether by binary pulsar observations, or by measurements of gravitational radiation from compact binary inspiral. Here we summarize the key results in a manner that parallels the results for GR.

\subsubsection{Scalar-tensor equations in "relaxed" form}

The field equations of scalar-tensor theory can be cast in a form similar to the "relaxed" equations of GR. Here one works in terms of an auxiliary metric $\tilde{g}_{\alpha \beta} \equiv \varphi g_{\alpha \beta}$, where $\varphi \equiv\left(\phi / \phi_{0}\right)$ and $\phi_{0}$ is the asymptotic value of the scalar field, and defines the auxiliary gothic inverse metric $\tilde{\mathfrak{g}}^{\alpha \beta} \equiv \sqrt{-\tilde{g}} \tilde{g}^{\alpha \beta}$, and the auxiliary tensor gravitational potential $\tilde{h}^{\alpha \beta} \equiv \eta^{\alpha \beta}-\tilde{\mathfrak{g}}^{\alpha \beta}$, along with the harmonic gauge condition $\partial \tilde{h}^{\alpha \beta} / \partial x^{\beta}=0$. The field equations then take the form

$$
\square \tilde{h}^{\alpha \beta}=-16 \pi G \tilde{\tau}^{\alpha \beta},
$$

where $\square \equiv-\partial^{2} / \partial t^{2}+\nabla^{2}$ is again the flat-spacetime wave operator, and where

$$
\tilde{\tau}^{\alpha \beta}=(-\tilde{g})\left(\frac{\varphi}{\phi_{0}} T^{\alpha \beta}+\tilde{t}_{\phi}^{\alpha \beta}+\tilde{t}_{\mathrm{LL}}^{\alpha \beta}+\tilde{t}_{\mathrm{H}}^{\alpha \beta}\right),
$$

where $\tilde{t}_{\phi}^{\alpha \beta} \equiv(3+2 \omega) \varphi^{-2} \varphi_{, \mu} \varphi_{, \nu}\left(\tilde{g}^{\mu \alpha} \tilde{g}^{\nu \beta}-\frac{1}{2} \tilde{g}^{\mu \nu} \tilde{g}^{\alpha \beta}\right)$ is a scalar stress-energy tensor, and where $\tilde{t}_{\mathrm{LL}}^{\alpha \beta}$ and $\tilde{t}_{\mathrm{H}}^{\alpha \beta}$ have exactly the same forms, when written in terms of $\tilde{h}^{\alpha \beta}$, as their counterparts in GR do in terms of $h^{\alpha \beta}$. Note that this is equivalent to formulating the relaxed equations of scalar-tensor theory in the Einstein conformal frame. The field equation for the scalar field can be written in the form $\square \varphi=-8 \pi G \tilde{\tau}_{s}$, where $\tilde{\tau}_{s}$ is a source consisting of a matter term, a scalar energy density term and a term that mixes $\tilde{h}^{\alpha \beta}$ and $\varphi$ (see [286] for details).

In order to incorporate the internal gravity of compact, self-gravitating bodies, it is common to adopt an approach pioneered by Eardley [139], based in part on general arguments dating back to Dicke, in which one treats the matter energy-momentum tensor as a sum of delta functions located at the position of each body, but assumes that the mass of each body is a function $M_{A}(\phi)$ of the scalar field. This reflects the fact that the gravitational binding energy of the body is controlled by the value of the gravitational constant, which is directly related to the value of the background scalar field in which the body finds itself. The underlying assumption is that the timescale for orbital motion is long compared to the internal dynamical timescale of the body, so that the body's structure evolves adiabatically in response to the changing scalar field. Consequently, the matter action will have an effective dependence on $\phi$, and as a result the field equations will depend on the "sensitivity" of the mass of each body to variations in the scalar field, holding the total number of baryons fixed. The sensitivity of body $A$ is defined by

$$
s_{A} \equiv\left(\frac{d \ln M_{A}(\phi)}{d \ln \phi}\right)
$$

evaluated at a value of the scalar field far from the body. For neutron stars, the sensitivity depends on the mass and equation of state of the star and is typically of order 0.2 ; in the weak-field limit, $s_{A}$ is proportional to the Newtonian self-gravitational energy per unit mass of the body. From a theorem of Hawking [185], for stationary black holes, it is known that $s_{\mathrm{BH}}=1 / 2$. This means, among other things, that the source $\tilde{\tau}_{s}$ for the scalar field will contain an explicit term dependent upon $\partial T / \partial \phi$, because of the dependence on $M_{A}(\phi)$. 


\subsubsection{Equations of motion and gravitational waveform}

By following the methods of post-Minkowskian theory adapted to scalar-tensor theory, it has been possible to derive the equations of motion for binary systems of compact bodies to $2.5 \mathrm{PN}$ order [286] and the gravitational-wave signal and energy flux to 1PN order beyond the quadrupole approximation. Here we shall quote selected results in parallel with those quoted in Section 5.3.2. The relative two-body equation of motion has the form

$$
\mathbf{a}=\frac{d \mathbf{v}}{d t}=\frac{\alpha m}{r^{2}}\left\{-\hat{\mathbf{n}}+\mathbf{A}_{1 \mathrm{PN}}+\mathbf{A}_{1.5 \mathrm{PN}}+\mathbf{A}_{2 \mathrm{PN}}+\mathbf{A}_{2.5 \mathrm{PN}}+\mathbf{A}_{3 \mathrm{PN}}+\mathbf{A}_{3.5 \mathrm{PN}}+\ldots\right\} .
$$

The key difference between this PN series and that in GR is the presence of a radiation-reaction term at $1.5 \mathrm{PN}$ order, caused by the emission of dipole gravitational radiation. The key parameters that appear in the two-body equations of motion are given in Table 6 . Notice that $\alpha$ plays the role of a two-body gravitational interaction parameter; $\bar{\gamma}$ and $\bar{\beta}_{A}$ are the two-body versions of $\gamma-1$ and $\beta-1$ respectively. In the limit of weakly self-gravitating bodies $\left(s_{A} \rightarrow 0\right), \alpha \rightarrow 1, \bar{\gamma} \rightarrow \gamma-1=-2 \zeta$ and $\bar{\beta}_{A} \rightarrow \beta-1=\zeta \lambda($ compare with Table 3$)$.

Table 6: Parameters used in the equations of motion.

\begin{tabular}{cl}
\hline Parameter & Definition \\
\hline \multicolumn{3}{c}{ Scalar-tensor parameters } \\
$\zeta$ & $1 /\left(4+2 \omega_{0}\right)$ \\
$\lambda$ & $(d \omega / d \varphi)_{0} \zeta^{2} /(1-\zeta)^{2}$ \\
Sensitivities & \\
$s_{A}$ & {$\left[d \ln M_{A}(\phi) / d \ln \phi\right]_{0}$} \\
$s_{A}^{\prime}$ & {$\left[d^{2} \ln M_{A}(\phi) / d \ln \phi^{2}\right]_{0}$} \\
Equation of & motion parameters \\
$\alpha$ & $1-\zeta+\zeta\left(1-2 s_{1}\right)\left(1-2 s_{2}\right)$ \\
$\bar{\gamma}$ & $-2 \alpha^{-1} \zeta\left(1-2 s_{1}\right)\left(1-2 s_{2}\right)$ \\
$\bar{\beta}_{1}$ & $\alpha^{-2} \zeta\left(1-2 s_{2}\right)^{2}\left(\lambda\left(1-2 s_{1}\right)+2 \zeta s_{1}^{\prime}\right)$ \\
$\bar{\beta}_{2}$ & $\alpha^{-2} \zeta\left(1-2 s_{1}\right)^{2}\left(\lambda\left(1-2 s_{2}\right)+2 \zeta s_{2}^{\prime}\right)$ \\
\hline
\end{tabular}

Here we quote only the $1 \mathrm{PN}$ corrections and the leading radiation-reaction terms at $1.5 \mathrm{PN}$ and 2.5PN order

$$
\begin{aligned}
\mathbf{A}_{1 \mathrm{PN}}= & \left\{\left(4+2 \eta+2 \bar{\gamma}+2 \bar{\beta}_{+}-2 \psi \bar{\beta}_{-}\right) \frac{\alpha m}{r}-(1+3 \eta+\bar{\gamma}) v^{2}+\frac{3}{2} \eta \dot{r}^{2}\right\} \hat{\mathbf{n}} \\
& +(4-2 \eta+2 \bar{\gamma}) \dot{r} \mathbf{v}, \\
\mathbf{A}_{1.5 \mathrm{PN}}= & \frac{4}{3} \eta \frac{\alpha m}{r} \zeta \mathcal{S}_{-}^{2}(3 \dot{r} \hat{\mathbf{n}}-\mathbf{v}), \\
\mathbf{A}_{2.5 \mathrm{PN}}= & \frac{8}{5} \eta \frac{\alpha m}{r}\left\{\left(a_{1} v^{2}+a_{2} \frac{\alpha m}{r}+a_{3} \dot{r}^{2}\right) \dot{r} \hat{\mathbf{n}}-\left(b_{1} v^{2}+b_{2} \frac{m}{r}+b_{3} \dot{r}^{2}\right) \mathbf{v}\right\},
\end{aligned}
$$


where

$$
\begin{aligned}
a_{1}= & 3-\frac{5}{2} \bar{\gamma}+\frac{15}{2} \bar{\beta}_{+}+\frac{5}{8} \zeta \mathcal{S}_{-}^{2}(9+4 \bar{\gamma}-2 \eta)+\frac{15}{8} \zeta \psi \mathcal{S}_{-} \mathcal{S}_{+}, \\
a_{2}= & \frac{17}{3}+\frac{35}{6} \bar{\gamma}-\frac{95}{6} \bar{\beta}_{+}-\frac{5}{24} \zeta \mathcal{S}_{-}^{2}\left[135+56 \bar{\gamma}+8 \eta+32 \bar{\beta}_{+}\right]+30 \zeta \mathcal{S}_{-}\left(\frac{\mathcal{S}_{-} \bar{\beta}_{+}+\mathcal{S}_{+} \bar{\beta}_{-}}{\bar{\gamma}}\right) \\
& -\frac{5}{8} \zeta \psi \mathcal{S}_{-}\left(\mathcal{S}_{+}-\frac{32}{3} \mathcal{S}_{-} \bar{\beta}_{-}+16 \frac{\mathcal{S}_{+} \bar{\beta}_{+}+\mathcal{S}_{-} \bar{\beta}_{-}}{\bar{\gamma}}\right)-40 \zeta\left(\frac{\mathcal{S}_{+} \bar{\beta}_{+}+\mathcal{S}_{-} \bar{\beta}_{-}}{\bar{\gamma}}\right)^{2}, \\
a_{3}= & \frac{25}{8}\left[2 \bar{\gamma}-\zeta \mathcal{S}_{-}^{2}(1-2 \eta)-4 \bar{\beta}_{+}-\zeta \psi \mathcal{S}_{-} \mathcal{S}_{+}\right], \\
b_{1}= & 1-\frac{5}{6} \bar{\gamma}+\frac{5}{2} \bar{\beta}_{+}-\frac{5}{24} \zeta \mathcal{S}_{-}^{2}(7+4 \bar{\gamma}-2 \eta)+\frac{5}{8} \zeta \psi \mathcal{S}_{-} \mathcal{S}_{+}, \\
b_{2}= & 3+\frac{5}{2} \bar{\gamma}-\frac{5}{2} \bar{\beta}_{+}-\frac{5}{24} \zeta \mathcal{S}_{-}^{2}\left[23+8 \bar{\gamma}-8 \eta+8 \bar{\beta}_{+}\right]+\frac{10}{3} \zeta \mathcal{S}_{-}\left(\frac{\mathcal{S}_{-} \bar{\beta}_{+}+\mathcal{S}_{+} \bar{\beta}_{-}}{\bar{\gamma}}\right) \\
& -\frac{5}{8} \zeta \psi \mathcal{S}_{-}\left(\mathcal{S}_{+}-\frac{8}{3} \mathcal{S}_{-} \bar{\beta}_{-}+\frac{16}{3} \frac{\mathcal{S}_{+} \bar{\beta}_{+}+\mathcal{S}_{-} \bar{\beta}_{-}}{\bar{\gamma}}\right), \\
b_{3}= & \frac{5}{8}\left[6 \bar{\gamma}+\zeta \mathcal{S}_{-}^{2}(13+8 \bar{\gamma}+2 \eta)-12 \bar{\beta}_{+}-3 \zeta \psi \mathcal{S}_{-} \mathcal{S}_{+}\right]
\end{aligned}
$$

where

$$
\begin{aligned}
\bar{\beta}_{ \pm} & \equiv \frac{1}{2}\left(\bar{\beta}_{1} \pm \bar{\beta}_{2}\right), \\
\psi & \equiv\left(m_{1}-m_{2}\right) / m, \\
\mathcal{S}_{-} & \equiv-\alpha^{-1 / 2}\left(s_{1}-s_{2}\right), \\
\mathcal{S}_{+} & \equiv \alpha^{-1 / 2}\left(1-s_{1}-s_{2}\right) .
\end{aligned}
$$

The periastron advance that results from these equations is given by

$$
\dot{\omega}=\frac{6 \pi \alpha m f_{b}}{a\left(1-e^{2}\right)}\left[1+\frac{2 \bar{\gamma}-\bar{\beta}_{+}-\psi \bar{\beta}_{-}}{3}\right] .
$$

where $2 \pi f_{b}=\left(\alpha m / a^{3}\right)^{1 / 2}$.

The tensor part of the gravitational waveform has the schematic form

$$
\tilde{h}^{i j}=\frac{2(1-\zeta) m}{R}\left\{Q^{i j}+Q_{0.5 \mathrm{PN}}^{i j}+Q_{1 \mathrm{PN}}^{i j}+Q_{1.5 \mathrm{PN}}^{i j}+Q_{2 \mathrm{PN}}^{i j}+\ldots\right\},
$$

where

$$
Q^{i j}=2 \eta\left(v^{i} v^{j}-\frac{\alpha m \hat{n}^{i} \hat{n}^{j}}{r}\right)
$$

Contributions to the tensor waveform through $2 \mathrm{PN}$ order have been derived by Lang [241]. The scalar waveform is given by $\phi=\phi_{0}(1+\Psi)$, where,

$$
\Psi=\zeta \eta \alpha^{1 / 2} \frac{m}{R}\left\{\Psi_{-0.5 \mathrm{PN}}+\Psi_{0 \mathrm{PN}}+\Psi_{0.5 \mathrm{PN}}+\Psi_{1 \mathrm{PN}}+\ldots\right\},
$$


where, ignoring terms that are constant in time,

$$
\begin{aligned}
\Psi_{-0.5 \mathrm{PN}}= & 4 \mathcal{S}_{-}(\hat{\mathbf{N}} \cdot \mathbf{v}) \\
\Psi_{0 \mathrm{PN}}=2\left(\mathcal{S}_{+}-\psi \mathcal{S}_{-}\right)\left[(\hat{\mathbf{N}} \cdot \mathbf{v})^{2}-\frac{\alpha m}{r}(\hat{\mathbf{N}} \cdot \mathbf{x})^{2}\right] & \\
& -2 \frac{\alpha m}{r}\left[3 \mathcal{S}_{+}-\psi \mathcal{S}_{-}-8\left(\frac{\mathcal{S}_{+} \bar{\beta}_{+}+\mathcal{S}_{-} \bar{\beta}_{-}}{\bar{\gamma}}\right)\right], \\
\Psi_{0.5 \mathrm{PN}}=- & \frac{\partial}{\partial t}\left\{(\hat{\mathbf{N}} \cdot \mathbf{x})\left[(3-4 \eta) \mathcal{S}_{-}-\psi \mathcal{S}_{+}+8 \psi\left(\frac{\mathcal{S}_{+} \bar{\beta}_{+}+\mathcal{S}_{-} \bar{\beta}_{-}}{\bar{\gamma}}\right)-8\left(\frac{\mathcal{S}_{-} \bar{\beta}_{+}+\mathcal{S}_{+} \bar{\beta}_{-}}{\bar{\gamma}}\right)\right]\right\} \\
& +\frac{1}{3}\left[(1-2 \eta) \mathcal{S}_{-}-\psi \mathcal{S}_{+}\right] \frac{\partial^{3}}{\partial t^{3}}(\hat{\mathbf{N}} \cdot \mathbf{x})^{3},
\end{aligned}
$$

where $\hat{\mathbf{N}}$ is a unit vector directed toward the observer.

The energy flux is given by

$$
d E / d t=-\frac{4}{3} \zeta \frac{\mu \eta}{r}\left(\frac{\alpha m}{r}\right)^{3} \mathcal{S}_{-}^{2}-\frac{8}{15} \frac{\mu \eta}{r}\left(\frac{\alpha m}{r}\right)^{3}\left(\kappa_{1} v^{2}-\kappa_{2} \dot{r}^{2}\right)
$$

where the first term is the contribution of dipole radiation (formally of $-1 \mathrm{PN}$ order), and the second term (formally of 0PN order, according to the conventional rules of counting) is a combination of quadrupole radiation, $\mathrm{PN}$ corrections to monopole and dipole radiation, and even a cross-term between dipole and octupole radiation. The coefficients $\kappa_{1}$ and $\kappa_{2}$ are given by [286]

$$
\begin{aligned}
\kappa_{1}= & 12+5 \bar{\gamma}-5 \zeta \mathcal{S}_{-}^{2}\left(3+\bar{\gamma}+2 \bar{\beta}_{+}\right)+10 \zeta \mathcal{S}_{-}\left(\frac{\mathcal{S}_{-} \bar{\beta}_{+}+\mathcal{S}_{+} \bar{\beta}_{-}}{\bar{\gamma}}\right) \\
& +10 \zeta \psi \mathcal{S}_{-}^{2} \bar{\beta}_{-}-10 \zeta \psi \mathcal{S}_{-}\left(\frac{\mathcal{S}_{+} \bar{\beta}_{+}+\mathcal{S}_{-} \bar{\beta}_{-}}{\bar{\gamma}}\right), \\
\kappa_{2}= & 11+\frac{45}{4} \bar{\gamma}-40 \bar{\beta}_{+}-5 \zeta \mathcal{S}_{-}^{2}\left[17+6 \bar{\gamma}+\eta+8 \bar{\beta}_{+}\right]+90 \zeta \mathcal{S}_{-}\left(\frac{\mathcal{S}_{-} \bar{\beta}_{+}+\mathcal{S}_{+} \bar{\beta}_{-}}{\bar{\gamma}}\right) \\
& +40 \zeta \psi \mathcal{S}_{-}^{2} \bar{\beta}_{-}-30 \zeta \psi \mathcal{S}_{-}\left(\frac{\mathcal{S}_{+} \bar{\beta}_{+}+\mathcal{S}_{-} \bar{\beta}_{-}}{\bar{\gamma}}\right)-120 \zeta\left(\frac{\mathcal{S}_{+} \bar{\beta}_{+}+\mathcal{S}_{-} \bar{\beta}_{-}}{\bar{\gamma}}\right)^{2} .
\end{aligned}
$$

These results are in complete agreement with the total energy flux to $-1 \mathrm{PN}$ and $0 \mathrm{PN}$ orders, as calculated by Damour and Esposito-Farèse [102]. They disagree with the flux formula of Will and Zaglauer [434], as repeated in earlier versions of this Living Review as well as in [7]. Will and Zaglauer [434] failed to take into account PN corrections to the dipole term induced by PN corrections in the equations of motion, and a dipole-octupole cross term in the scalar energy flux, all of which contribute to the flux at the same 0PN order as the quadrupole and monopole contributions.

In the limit of weakly self-gravitating bodies the equations of motion and energy flux (including the dipole term) reduce to the standard results quoted in TEGP [420].

\subsubsection{Binary systems containing black holes}

Roger Penrose was probably the first to conjecture, in a talk at the 1970 Fifth Texas Symposium, that black holes in Brans-Dicke theory are identical to their GR counterparts [387]. Motivated by this remark, Thorne and Dykla showed that during gravitational collapse to form a black hole, the Brans-Dicke scalar field is radiated away, in accord with Price's theorem, leaving only its constant asymptotic value, and a GR black hole [387]. Hawking [185] proved on general grounds that stationary, asymptotically flat black holes in vacuum in BD are the black holes of GR. The 
basic idea is that black holes in vacuum with non-singular event horizons cannot support scalar "hair". Hawking's theorem was extended to the class of $f(R)$ theories that can be transformed into generalized scalar-tensor theories by Sotiriou and Faraoni [371].

A consequence of these theorems is that, for a stationary black hole, $s=1 / 2$. Another way to see this is to note that, because all information about the matter that formed the black hole has vanished behind the event horizon, the only scale on which the mass of the hole can depend is the Planck scale, and thus $M \propto M_{\text {Planck }} \propto G^{-1 / 2} \propto \phi^{1 / 2}$. Hence $s=1 / 2$.

If both bodies in the binary system are black holes, then setting $s_{A}=1 / 2$ for each body, all the parameters $\bar{\gamma}, \bar{\beta}_{A}$ and $\mathcal{S}_{ \pm}$vanish identically, and $\alpha=1-\zeta$. But since $\alpha$ appears only in the combination with $\alpha m$, a simple rescaling of each mass puts all equations into complete agreement with those of GR. This is also true for the $2 \mathrm{PN}$ terms in the equations of motion [286]. Thus, in the class of scalar-tensor theories discussed here, binary black holes are observationally indistinguishable from their GR counterparts, at least to high orders in a PN approximation. It has also been shown, in the extreme mass-ratio limit to first order in the small mass, but to all PN orders, that binary black holes do not emit dipole gravitational radiation [450].

If one of the members of the binary system, say body 2 , is a black hole, with $s_{2}=1 / 2$, then $\alpha=1-\zeta, \bar{\gamma}=\bar{\beta}_{A}=0$, and hence, through $1 \mathrm{PN}$ order, the motion is again identical to that in GR. At 1.5PN order, dipole radiation reaction kicks in, since $s_{1}<1 / 2$. In this case, $\mathcal{S}_{-}=\mathcal{S}_{+}=\alpha^{-1 / 2}\left(1-2 s_{1}\right) / 2$, and thus the $1.5 \mathrm{PN}$ coefficients in the relative equation of motion (94) take the form

$$
\begin{aligned}
& A_{1.5 \mathrm{PN}}=\frac{5}{8} Q, \\
& B_{1.5 \mathrm{PN}}=\frac{5}{24} Q,
\end{aligned}
$$

where

$$
Q \equiv \frac{\zeta}{1-\zeta}\left(1-2 s_{1}\right)^{2}=\frac{1}{3+2 \omega_{0}}\left(1-2 s_{1}\right)^{2},
$$

while the coefficients in the energy loss rate simplify to

$$
\begin{aligned}
& \kappa_{1}=12-\frac{15}{4} Q, \\
& \kappa_{2}=11-\frac{5}{4} Q(17+\eta) .
\end{aligned}
$$

The result is that the motion of a mixed compact binary system through 2.5PN order differs from its general relativistic counterpart only by terms that depend on a single parameter $Q$, as defined by Eq. (106).

It should be pointed out that there are ways to induce scalar hair on a black hole. One is to introduce a potential $V(\phi)$, which, depending on its form, can help to support a non-trivial scalar field outside a black hole. Another is to introduce matter. A companion neutron star is an obvious choice, and such a binary system in scalar-tensor theory is clearly different from its general relativistic counterpart. Another possibility is a distribution of cosmological matter that can support a time-varying scalar field at infinity. This possibility has been called "Jacobson's miracle hair-growth formula" for black holes, based on work by Jacobson [202, 191]. 


\section{Stellar System Tests of Gravitational Theory}

\subsection{The binary pulsar and general relativity}

The 1974 discovery of the binary pulsar B1913+16 by Joseph Taylor and Russell Hulse during a routine search for new pulsars provided the first possibility of probing new aspects of gravitational theory: the effects of strong relativistic internal gravitational fields on orbital dynamics, and the effects of gravitational radiation reaction. For reviews of the discovery, see the published Nobel Prize lectures by Hulse and Taylor [195, 385]. For reviews of the current status of testing general relativity with pulsars, including binary and millisecond pulsars, see [261, 374, 412]; specific details on every pulsar discovered to date, along with orbit elements of pulsars in binary systems, can be found at the Australia Telescope National Facility (ATNF) online pulsar catalogue [28]. Table 7 lists the current values of the key orbital and relativistic parameters for B1913+16, from analysis of data through 2006 [409].

Table 7: Parameters of the binary pulsar B1913+16. The numbers in parentheses denote errors in the last digit. Data taken from [409]. Note that $\gamma^{\prime}$ is not the same as the PPN parameter $\gamma$ [see Eqs. (108)].

\begin{tabular}{lll}
\hline Parameter & \multicolumn{1}{c}{$\begin{array}{c}\text { Symbol } \\
\text { (units) }\end{array}$} & \multicolumn{1}{c}{ Value } \\
\hline (i) Astrometric and spin parameters: & & \\
Right Ascension & $\alpha$ & $19^{\mathrm{h}} 15^{\mathrm{m}} 27 .^{\mathrm{s}} 99928(9)$ \\
Declination & $\delta$ & $16^{\circ} 06^{\prime} 27 .^{\prime \prime} 3871(13)$ \\
Pulsar period & $P_{\mathrm{p}}(\mathrm{ms})$ & $59.0299983444181(5)$ \\
Derivative of period & $\dot{P}_{\mathrm{p}}$ & $8.62713(8) \times 10^{-18}$ \\
(ii) "Keplerian" parameters: & & \\
Projected semimajor axis & $a_{\mathrm{p}} \sin i(\mathrm{~s})$ & $2.341782(3)$ \\
Eccentricity & $e$ & $0.6171334(5)$ \\
Orbital period & $P_{\mathrm{b}}(\mathrm{day})$ & $0.322997448911(4)$ \\
Longitude of periastron & $\omega_{0}\left(^{\circ}\right)$ & $292.54472(6)$ \\
Julian date of periastron & $T_{0}(\mathrm{MJD})$ & $52144.90097841(4)$ \\
(iii) "Post-Keplerian" parameters: & & \\
Mean rate of periastron advance & $\langle\dot{\omega}\rangle\left(^{\circ} \mathrm{yr}{ }^{-1}\right)$ & $4.226598(5)$ \\
Redshift/time dilation & $\gamma^{\prime}(\mathrm{ms})$ & $4.2992(8)$ \\
Orbital period derivative & $\dot{P}_{\mathrm{b}}\left(10^{-12}\right)$ & $-2.423(1)$ \\
\hline
\end{tabular}

The system consists of a pulsar of nominal period $59 \mathrm{~ms}$ in a close binary orbit with an unseen companion. The orbital period is about 7.75 hours, and the eccentricity is 0.617 . From detailed analyses of the arrival times of pulses (which amounts to an integrated version of the Doppler-shift methods used in spectroscopic binary systems), extremely accurate orbital and physical parameters for the system have been obtained (see Table 7$)$. Because the orbit is so close $\left(\approx 1 R_{\odot}\right)$ and because there is no evidence of an eclipse of the pulsar signal or of mass transfer from the companion, it is generally agreed that the companion is compact. Evolutionary arguments suggest that it is most likely a dead pulsar, while B1913+16 is a "recycled" pulsar. Thus the orbital motion is very clean, free from tidal or other complicating effects. Furthermore, the data acquisition is "clean" in the sense that by exploiting the intrinsic stability of the pulsar clock combined with the 
ability to maintain and transfer atomic time accurately using GPS, the observers can keep track of pulse time-of-arrival with an accuracy of $13 \mu \mathrm{s}$, despite extended gaps between observing sessions (including a several-year gap in the middle 1990s for an upgrade of the Arecibo radio telescope). The pulsar has experienced only one small "glitch" in its pulse period, in May 2003.

Three factors made this system an arena where relativistic celestial mechanics must be used: the relatively large size of relativistic effects $\left[v_{\text {orbit }} \approx(m / r)^{1 / 2} \approx 10^{-3}\right]$, a factor of 10 larger than the corresponding values for solar-system orbits; the short orbital period, allowing secular effects to build up rapidly; and the cleanliness of the system, allowing accurate determinations of small effects. Because the orbital separation is large compared to the neutron stars' compact size, tidal effects can be ignored. Just as Newtonian gravity is used as a tool for measuring astrophysical parameters of ordinary binary systems, so GR is used as a tool for measuring astrophysical parameters in the binary pulsar.

The observational parameters that are obtained from a least-squares solution of the arrival-time data fall into three groups:

1. non-orbital parameters, such as the pulsar period and its rate of change (defined at a given epoch), and the position of the pulsar on the sky;

2. five "Keplerian" parameters, most closely related to those appropriate for standard Newtonian binary systems, such as the eccentricity $e$, the orbital period $P_{\mathrm{b}}$, and the semi-major axis of the pulsar projected along the line of sight, $a_{\mathrm{p}} \sin i$; and

3. five "post-Keplerian" parameters.

The five post-Keplerian parameters are: $\langle\dot{\omega}\rangle$, the average rate of periastron advance; $\gamma^{\prime}$, the amplitude of delays in arrival of pulses caused by the varying effects of the gravitational redshift and time dilation as the pulsar moves in its elliptical orbit at varying distances from the companion and with varying speeds; $\dot{P}_{\mathrm{b}}$, the rate of change of orbital period, caused predominantly by gravitational radiation damping; and $r$ and $s=\sin i$, respectively the "range" and "shape" of the Shapiro time delay of the pulsar signal as it propagates through the curved spacetime region near the companion, where $i$ is the angle of inclination of the orbit relative to the plane of the sky. An additional 14 relativistic parameters are measurable in principle [119].

In GR, the five post-Keplerian parameters can be related to the masses of the two bodies and to measured Keplerian parameters by the equations (TEGP 12.1, 14.6 (a) [420])

$$
\begin{aligned}
\langle\dot{\omega}\rangle & =6 \pi f_{\mathrm{b}}\left(2 \pi m f_{\mathrm{b}}\right)^{2 / 3}\left(1-e^{2}\right)^{-1}, \\
\gamma^{\prime} & =e\left(2 \pi f_{\mathrm{b}}\right)^{-1}\left(2 \pi m f_{\mathrm{b}}\right)^{2 / 3} \frac{m_{2}}{m}\left(1+\frac{m_{2}}{m}\right), \\
\dot{P}_{\mathrm{b}} & =-\frac{192 \pi}{5}\left(2 \pi \mathcal{M} f_{\mathrm{b}}\right)^{5 / 3} F(e), \\
r & =m_{2}, \\
s & =\sin i,
\end{aligned}
$$

where $m_{1}$ and $m_{2}$ denote the pulsar and companion masses, respectively. The formula for $\langle\dot{\omega}\rangle$ ignores possible non-relativistic contributions to the periastron shift, such as tidally or rotationally induced effects caused by the companion (for discussion of these effects, see TEGP 12.1 (c) [420]). The formula for $\dot{P}_{\mathrm{b}}$ includes only quadrupole gravitational radiation; it ignores other sources of energy loss, such as tidal dissipation (TEGP 12.1 (f) [420]). Notice that, by virtue of Kepler's third law, $\left(2 \pi f_{\mathrm{b}}\right)^{2}=m / a^{3},\left(2 \pi m f_{\mathrm{b}}\right)^{2 / 3}=m / a \sim \epsilon$, thus the first two post-Keplerian parameters can be seen as $\mathcal{O}(\epsilon)$, or $1 \mathrm{PN}$ corrections to the underlying variable, while the third is an $\mathcal{O}\left(\epsilon^{5 / 2}\right)$, or 2.5PN correction. The current observed values for the Keplerian and post-Keplerian parameters are shown in Table 7 . The parameters $r$ and $s$ are not separately measurable with interesting 
accuracy for B1913+16 because the orbit's $47^{\circ}$ inclination does not lead to a substantial Shapiro delay. However they are measurable in the double pulsar, for example.

Because $f_{\mathrm{b}}$ and $e$ are separately measured parameters, the measurement of the three postKeplerian parameters provides three constraints on the two unknown masses. The periastron shift measures the total mass of the system, $\dot{P}_{\mathrm{b}}$ measures the chirp mass, and $\gamma^{\prime}$ measures a complicated function of the masses. GR passes the test if it provides a consistent solution to these constraints, within the measurement errors.

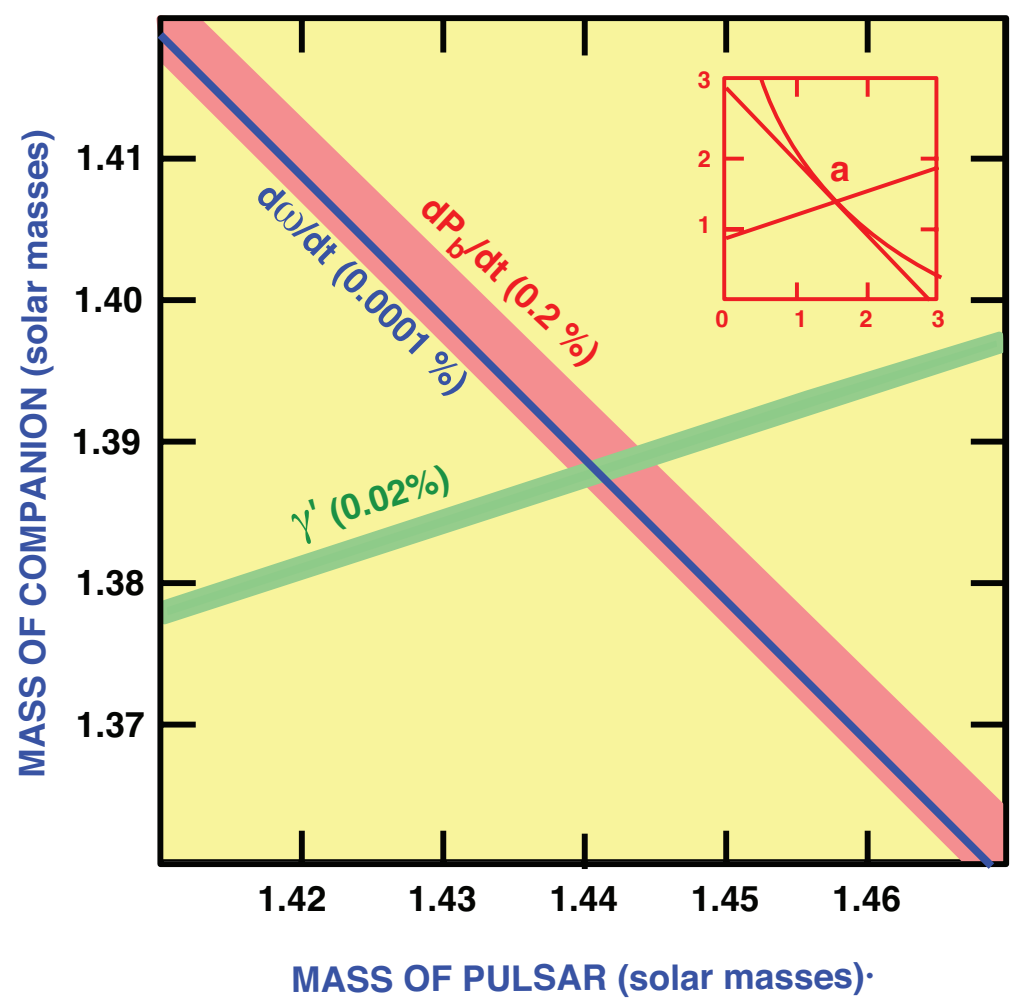

Figure 6: Constraints on masses of the pulsar and its companion from data on B1913+16, assuming GR to be valid. The width of each strip in the plane reflects observational accuracy, shown as a percentage. An inset shows the three constraints on the full mass plane; the intersection region (a) has been magnified 400 times for the full figure.

From the intersection of the $\langle\dot{\omega}\rangle$ and $\gamma^{\prime}$ constraints we obtain the values $m_{1}=1.4398 \pm$ $0.0002 M_{\odot}$ and $m_{2}=1.3886 \pm 0.0002 M_{\odot}$. The third of Eqs. (108) then predicts the value $\dot{P}_{\mathrm{b}}=-2.402531 \pm 0.000014 \times 10^{-12}$. In order to compare the predicted value for $\dot{P}_{\mathrm{b}}$ with the observed value of Table 7 , it is necessary to take into account the small kinematic effect of a relative acceleration between the binary pulsar system and the solar system caused by the differential rotation of the galaxy. Using data on the location and proper motion of the pulsar, combined with the best information available on galactic rotation; the current value of this effect is $\dot{P}_{\mathrm{b}}^{\text {gal }} \simeq-(0.027 \pm 0.005) \times 10^{-12}$. Subtracting this from the observed $\dot{P}_{\mathrm{b}}$ (see Table 7$)$ gives the corrected $\dot{P}_{\mathrm{b}}^{\text {corr }}=-(2.396 \pm 0.005) \times 10^{-12}$, which agrees with the prediction within the errors. In other words,

$$
\frac{\dot{P}_{\mathrm{b}}^{\mathrm{corr}}}{\dot{P}_{\mathrm{b}}^{\mathrm{GR}}}=0.997 \pm 0.002
$$


The consistency among the measurements is displayed in Figure 6, in which the regions allowed by the three most precise constraints have a single common overlap. Uncertainties in the parameters that go into the galactic correction are now the limiting factor in the accuracy of the test of gravitational damping.

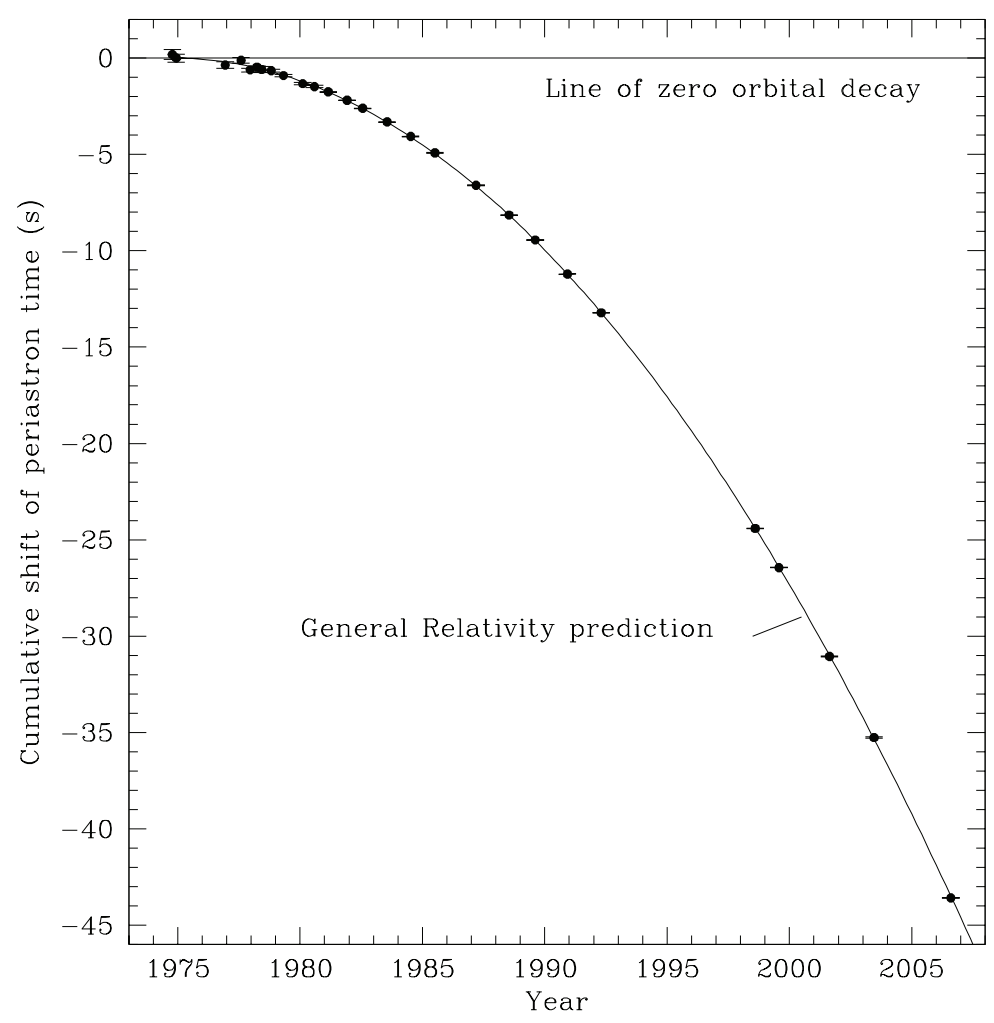

Figure 7: Plot of the cumulative shift of the periastron time from 1975-2005. The points are data, the curve is the GR prediction. The gap during the middle 1990s was caused by a closure of Arecibo for upgrading. Image reproduced with permission from [409], copyright by AAS.

A third way to display the agreement with GR is by comparing the observed phase of the orbit with a theoretical template phase as a function of time. If $f_{\mathrm{b}}$ varies slowly in time, then to first order in a Taylor expansion, the orbital phase is given by $\Phi_{\mathrm{b}}(t)=2 \pi f_{\mathrm{b} 0} t+\pi \dot{f}_{\mathrm{b} 0} t^{2}$. The time of periastron passage $t_{\mathrm{P}}$ is given by $\Phi\left(t_{\mathrm{P}}\right)=2 \pi N$, where $N$ is an integer, and consequently, the periastron time will not grow linearly with $N$. Thus the cumulative difference between periastron time $t_{\mathrm{P}}$ and $N / f_{\mathrm{b} 0}$, the quantities actually measured in practice, should vary according to $t_{\mathrm{P}}-N / f_{\mathrm{b} 0}=$ $-\dot{f}_{\mathrm{b} 0} N^{2} / 2 f_{\mathrm{b} 0}^{3} \approx-\left(\dot{f}_{\mathrm{b} 0} / 2 f_{\mathrm{b} 0}\right) t^{2}$. Figure 7 shows the results: The dots are the data points, while the curve is the predicted difference using the measured masses and the quadrupole formula for $\dot{f}_{\mathrm{b} 0}[409]$.

The consistency among the constraints provides a test of the assumption that the two bodies behave as "point" masses, without complicated tidal effects, obeying the general relativistic equations of motion including gravitational radiation. It is also a test of strong gravity, in that the highly relativistic internal structure of the neutron stars does not influence their orbital motion, as predicted by the SEP of GR. 
Observations [231, 410] indicate that the pulse profile is varying with time, which suggests that the pulsar is undergoing geodetic precession on a 300-year timescale as it moves through the curved spacetime generated by its companion (see Section 4.4.2). The amount is consistent with GR, assuming that the pulsar's spin is suitably misaligned with the orbital angular momentum. Unfortunately, the evidence suggests that the pulsar beam may precess out of our line of sight by 2025 .

\subsection{A zoo of binary pulsars}

More than 70 binary neutron star systems with orbital periods less than a day are now known. While some are less interesting for testing relativity, some have yielded interesting tests, and others, notably the recently discovered "double pulsar" are likely to continue to produce significant results well into the future. Here we describe some of the more interesting or best studied cases;

The "double" pulsar: J0737-3039A, B. This binary pulsar system, discovered in 2003 [72], was already remarkable for its extraordinarily short orbital period ( 0.1 days) and large periastron advance $\left(16.8995^{\circ} \mathrm{yr}^{-1}\right)$, but then the companion was also discovered to be a pulsar [265]. Because two projected semi-major axes could be measured, the mass ratio was obtained directly from the ratio of the two values of $a_{\mathrm{p}} \sin i$, and thereby the two masses could be obtained by combining that ratio with the periastron advance, assuming GR. The results are $m_{A}=1.3381 \pm 0.0007 M_{\odot}$ and $m_{B}=1.2489 \pm 0.0007 M_{\odot}$, where $A$ denotes the primary (first) pulsar. From these values, one finds that the orbit is nearly edge-on, with $\sin i=0.9997$, a value which is completely consistent with that inferred from the Shapiro delay parameter. In fact, the five measured post-Keplerian parameters plus the ratio of the projected semi-major axes give six constraints on the masses (assuming GR): as seen in Figure 8, all six overlap within their measurement errors [232]. (Note that Figure 8 is based on more recent data than that quoted in [232], in this discussion and in Table 8.) Because of the location of the system, galactic proper-motion effects play a significantly smaller role in the interpretation of $\dot{P}_{\mathrm{b}}$ measurements than they did in B1913+16; this and the reduced effect of interstellar dispersion means that the accuracy of measuring the gravitational-wave damping may soon beat that from the Hulse-Taylor system. It may ultimately be necessary for the data analysis to include second post-Newtonian $(2 \mathrm{PN})$ corrections, for example in the pericenter advance. The geodetic precession of pulsar B's spin axis has also been measured by monitoring changes in the patterns of eclipses of the signal from pulsar A, with a result in agreement with GR to about 13 percent [68]; the constraint on the masses from that effect (assuming GR to be correct) is also shown in Figure 8. In fact, pulsar B has precessed so much that its signal no longer sweeps by the Earth, so it has gone "silent". For a recent overview of the double pulsar, see [71].

J1738+0333: A white-dwarf companion. This is a low-eccentricity, 8.5-hour period system in which the white-dwarf companion is bright enough to permit detailed spectroscopy, allowing the companion mass to be determined directly to be $0.181 M_{\odot}$. The mass ratio is determined from Doppler shifts of the spectral lines of the companion and of the pulsar period, giving the pulsar mass $1.46 M_{\odot}$. Ten years of observation of the system yielded both a measurement of the apparent orbital period decay, and enough information about its parallax and proper motion to account for the substantial kinematic effect to give a value of the intrinsic period decay of $\dot{P}_{\mathrm{b}}=(-25.9 \pm 3.2) \times 10^{-15} \mathrm{~s} \mathrm{~s}^{-1}$ in agreement with the predicted effect [164]. But because of the asymmetry of the system, the result also places a significant bound on the existence of dipole radiation, predicted by many alternative theories of gravity (see Section 6.3 below for discussion). Data from this system were also used to place the tight bound on the PPN parameter $\alpha_{1}$ shown in Table 4. 


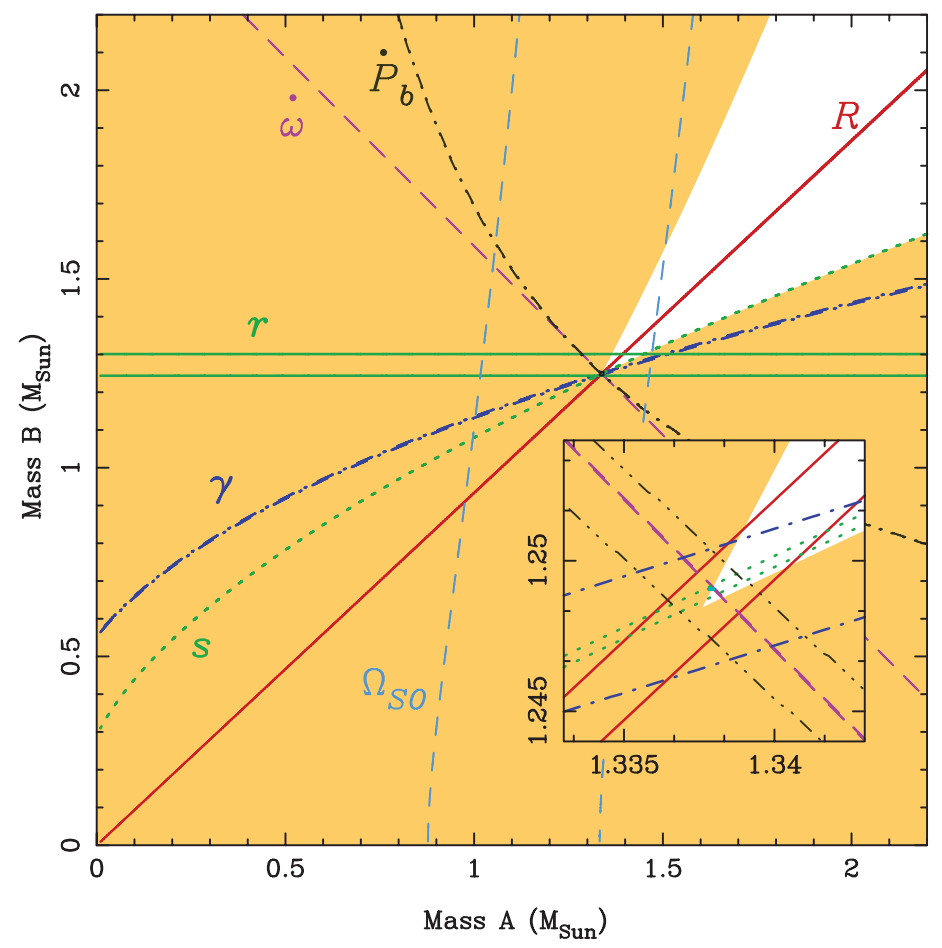

Figure 8: Constraints on masses of the pulsar and its companion from data on J0737-3039A,B, assuming GR to be valid. The inset shows the intersection region magnified by a factor of 80 . Image courtesy of M. Kramer.

J1141-6545: A white-dwarf companion. This system is similar in some ways to the HulseTaylor binary: short orbital period (0.20 days), significant orbital eccentricity $(0.172)$, rapid periastron advance (5.3 degrees per year) and massive components $\left(M_{p}=1.27 \pm 0.01 M_{\odot}, M_{c}=\right.$ $\left.1.02 \pm 0.01 M_{\odot}\right)$. The key difference is that the companion is again a white dwarf. The intrinsic orbit period decay has been measured in agreement with GR to about six percent, again placing limits on dipole gravitational radiation [46].

J0348+0432: The most massive neutron star. Discovered in 2011 [264, 19], this is another neutron-star white-dwarf system, in a very short period (0.1 day), low eccentricity $\left(2 \times 10^{-6}\right)$ orbit. Timing of the neutron star and spectroscopy of the white dwarf have led to mass values of $0.172 M_{\odot}$ for the white dwarf and $2.01 \pm 0.04 M_{\odot}$ for the pulsar, making it the most massive accurately measured neutron star yet. This supported an earlier discovery of a $2 M_{\odot}$ pulsar [127]; such large masses rule out a number of heretofore viable soft equations of state for nuclear matter. The orbit period decay agrees with the GR prediction within 20 percent and is expected to improve steadily with time.

J0337+1715: Two white-dwarf companions. This remarkable system was reported in 2014 [332]. It consists of a 2.73 millisecond pulsar $\left(M=1.44 M_{\odot}\right)$ with extremely good timing precision, accompanied by two white dwarfs in coplanar circular orbits. The inner white dwarf $\left(M=0.1975 M_{\odot}\right)$ has an orbital period of 1.629 days, with $e=6.918 \times 10^{-4}$, and the outer 
white dwarf $\left(M=0.41 M_{\odot}\right)$ has a period of 327.26 days, with $e=3.536 \times 10^{-2}$. This is an ideal system for testing the Nordtvedt effect in the strong-field regime. Here the inner system is the analogue of the Earth-Moon system, and the outer white dwarf plays the role of the Sun. Because the outer semi-major axis is about $1 / 3$ of an astronomical unit, the basic driving perturbation is comparable to that provided by the Sun. However, the self-gravitational binding energy per unit mass of the neutron star is almost a billion times larger than that of the Earth, greatly amplifying the size of the Nordtvedt effect. Depending on the details, this system could exceed lunar laser ranging in testing the Nordtvedt effect by several orders of magnitude.

Other binary pulsars. Two of the earliest binary pulsars, B1534+12 and B2127+11C, discovered in 1990, failed to live up to their early promise despite being similar to the Hulse-Taylor system in most respects (both were believed to be double neutron-star systems). The main reason was the significant uncertainty in the kinematic effect on $\dot{P}_{\mathrm{b}}$ of local accelerations, galactic in the case of B1534+12, and those arising from the globular cluster that was home to B2127+11C.

Table 8: Parameters of other binary pulsars. References may be found in the text. Values for orbit period derivatives include corrections for galactic kinematic effects

\begin{tabular}{llll}
\hline Parameter & J0737-3039(A, B) & J1738+0333 & J1141-6545 \\
\hline (i) Keplerian: & & & \\
$a_{\mathrm{p}} \sin i(\mathrm{~s})$ & $1.415032(1) / 1.516(2)$ & $0.34342913(2)$ & $1.858922(6)$ \\
$e$ & $0.0877775(9)$ & $(3.4 \pm 1.1) \times 10^{-7}$ & $0.171884(2)$ \\
$P_{\mathrm{b}}($ day $)$ & $0.10225156248(5)$ & $0.354790739872(1)$ & $0.1976509593(1)$ \\
& & & \\
(ii) Post-Keplerian: & & & $5.3096(4)$ \\
$\left.\langle\dot{\omega}\rangle{ }^{\circ} \mathrm{yr}^{-1}\right)$ & $16.8995(7)$ & & $0.77(1)$ \\
$\gamma^{\prime}(\mathrm{ms})$ & $0.386(3)$ & $-0.026(3)$ & $-0.401(25)$ \\
$\dot{P}_{\mathrm{b}}\left(10^{-12}\right)$ & $-1.25(2)$ & & \\
$r(\mu \mathrm{s})$ & $6.2(3)$ & & \\
$s=\sin i$ & $0.9997(4)$ & & \\
\hline
\end{tabular}

\subsection{Binary pulsars and alternative theories}

Soon after the discovery of the binary pulsar it was widely hailed as a new testing ground for relativistic gravitational effects. As we have seen in the case of GR, in most respects, the system has lived up to, indeed exceeded, the early expectations.

In another respect, however, the system has only partially lived up to its promise, namely as a direct testing ground for alternative theories of gravity. The origin of this promise was the discovery [139, 415] that alternative theories of gravity generically predict the emission of dipole gravitational radiation from binary star systems. In GR, there is no dipole radiation because the "dipole moment" (center of mass) of isolated systems is uniform in time (conservation of momentum), and because the "inertial mass" that determines the dipole moment is the same as the mass that generates gravitational waves (SEP). In other theories, while the inertial dipole moment may remain uniform, the "gravity wave" dipole moment need not, because the mass that generates gravitational waves depends differently on the internal gravitational binding energy of each body than does the inertial mass (violation of SEP). Schematically, in a coordinate system in which the center of inertial mass is at the origin, so that $m_{\mathrm{I}, 1} \mathbf{x}_{1}+m_{\mathrm{I}, 2} \mathbf{x}_{2}=0$, the dipole part 
of the retarded gravitational field would be given by

$$
h \sim \frac{1}{R} \frac{d}{d t}\left(m_{\mathrm{GW}, 1} \mathbf{x}_{1}+m_{\mathrm{GW}, 2} \mathbf{x}_{2}\right) \cdot \mathbf{N} \sim \frac{\eta m}{R} \mathbf{v} \cdot \mathbf{N}\left(\frac{m_{\mathrm{GW}, 1}}{m_{\mathrm{I}, 1}}-\frac{m_{\mathrm{GW}, 2}}{m_{\mathrm{I}, 2}}\right),
$$

where $\mathbf{v}=\mathbf{v}_{1}-\mathbf{v}_{2}, \mathbf{N}$ is a unit vector directed toward the observer, and $\eta$ and $m$ are defined using inertial masses. In theories that violate SEP, the difference between gravitational-wave mass and inertial mass is a function of the internal gravitational binding energy of the bodies. This additional form of gravitational radiation damping could, at least in principle, be significantly stronger than the usual quadrupole damping, because it depends on fewer powers of the orbital velocity $v$, and it depends on the gravitational binding energy per unit mass of the bodies, which, for neutron stars, could be as large as 20 percent (see TEGP 10 [420] for further details). As one fulfillment of this promise, Will and Eardley worked out in detail the effects of dipole gravitational radiation in the bimetric theory of Rosen, and, when the first observation of the decrease of the orbital period was announced in 1979, the Rosen theory suffered a terminal blow. A wide class of alternative theories also fails the binary pulsar test because of dipole gravitational radiation (TEGP 12.3 [420]).

On the other hand, the early observations of PSR 1913+16 already indicated that, in GR, the masses of the two bodies were nearly equal, so that, in theories of gravity that are in some sense "close" to GR, dipole gravitational radiation would not be a strong effect, because of the apparent symmetry of the system. The Rosen theory, and others like it, are not "close" to GR, except in their predictions for the weak-field, slow-motion regime of the solar system. When relativistic neutron stars are present, theories like these can predict strong effects on the motion of the bodies resulting from their internal highly relativistic gravitational structure (violations of SEP). As a consequence, the masses inferred from observations of the periastron shift and $\gamma^{\prime}$ may be significantly different from those inferred using GR, and may be different from each other, leading to strong dipole gravitational radiation damping. By contrast, the Brans-Dicke theory is "close" to GR, roughly speaking within $1 / \omega_{\mathrm{BD}}$ of the predictions of the latter, for large values of the coupling constant $\omega_{\mathrm{BD}}$. Thus, despite the presence of dipole gravitational radiation, the Hulse-Taylor binary pulsar provides at present only a weak test of pure Brans-Dicke theory, not competitive with solar-system tests.

However, the discovery of binary pulsar systems with a white dwarf companion, such as $\mathrm{J} 1738+0333, \mathrm{~J} 1141-6545$ and J0348+0432 has made it possible to perform strong tests of the existence of dipole radiation. This is because such systems are necessarily asymmetrical, since the gravitational binding energy per unit mass of white dwarfs is of order $10^{-4}$, much less than that of the neutron star. Already, significant bounds have been placed on dipole radiation using J1738+0333 and J1141-6545 [164, 46].

Because the gravitational-radiation and strong-field properties of alternative theories of gravity can be dramatically different from those of GR and each other, it is difficult to parametrize these aspects of the theories in the manner of the PPN framework. In addition, because of the generic violation of the strong equivalence principle in these theories, the results can be very sensitive to the equation of state and mass of the neutron star(s) in the system. In the end, there is no way around having to analyze every theory in turn. On the other hand, because of their relative simplicity, scalar-tensor theories provide an illustration of the essential effects, and so we shall discuss binary pulsars within this class of theories.

\subsection{Binary pulsars and scalar-tensor gravity}

Making the usual assumption that both members of the system are neutron stars, and using the methods summarized in TEGP 10-12 [420] (see also [286]) one can obtain formulas for the periastron shift, the gravitational redshift/second-order Doppler shift parameter, the Shapiro delay coefficients, and the rate of change of orbital period, analogous to Eqs. (108). These formulas 
depend on the masses of the two neutron stars, on their sensitivities $s_{A}$, and on the scalar-tensor parameters, as defined in Table 6 (and on a new sensitivity $\kappa^{*}$, defined below). First, there is a modification of Kepler's third law, given by

$$
2 \pi f_{\mathrm{b}}=\left(\frac{\alpha m}{a^{3}}\right)^{1 / 2}
$$

Then the predictions for $\langle\dot{\omega}\rangle, \gamma^{\prime}, \dot{P}_{\mathrm{b}}, r$ and $s$ are

$$
\begin{aligned}
\langle\dot{\omega}\rangle & =6 \pi f_{\mathrm{b}}\left(2 \pi \alpha m f_{\mathrm{b}}\right)^{2 / 3}\left(1-e^{2}\right)^{-1} \mathcal{P}, \\
\gamma^{\prime} & =e\left(2 \pi f_{\mathrm{b}}\right)^{-1}\left(2 \pi \alpha m f_{\mathrm{b}}\right)^{2 / 3} \frac{m_{2}}{m} \alpha^{-1}\left[1-2 \zeta s_{2}+\alpha \frac{m_{2}}{m}+\zeta \kappa_{1}^{*}\left(1-2 s_{2}\right)\right], \\
\dot{P}_{\mathrm{b}} & =-\frac{192 \pi}{5}\left(2 \pi \alpha \mathcal{M} f_{\mathrm{b}}\right)^{5 / 3} F^{\prime}(e)-8 \pi \zeta\left(2 \pi \mu f_{\mathrm{b}}\right) \mathcal{S}^{2} G(e), \\
r & =m_{2}(1-\zeta), \\
s & =\sin i,
\end{aligned}
$$

where

$$
\begin{aligned}
\mathcal{P} & =1+\frac{1}{3}\left(2 \bar{\gamma}-\bar{\beta}_{+}+\psi \bar{\beta}_{-}\right) \\
F^{\prime}(e) & =\frac{1}{12}\left(1-e^{2}\right)^{-7 / 2}\left[\kappa_{1}\left(1+\frac{7}{2} e^{2}+\frac{1}{2} e^{4}\right)-\frac{1}{2} \kappa_{2} e^{2}\left(1+\frac{1}{2} e^{2}\right)\right], \\
G(e) & =\left(1-e^{2}\right)^{-5 / 2}\left(1+\frac{1}{2} e^{2}\right)
\end{aligned}
$$

where $\kappa_{1}$ and $\kappa_{2}$ are defined in Eq. (104). The quantity $\kappa_{\mathrm{A}}^{*}$ is defined by

$$
\kappa_{\mathrm{A}}^{*}=\left(\frac{\partial\left(\ln I_{\mathrm{A}}\right)}{\partial(\ln \phi)}\right)
$$

and measures the "sensitivity" of the moment of inertia $I_{\mathrm{A}}$ of each body to changes in the scalar field for a fixed baryon number $N$ (see TEGP 11, 12 and 14.6 (c) [420] for further details). The sensitivities $s_{A}$ and $\kappa_{A}^{*}$ will depend on the neutron-star equation of state. Notice how the violation of SEP in scalar-tensor theory introduces complex structure-dependent effects in everything from the Newtonian limit (modification of the effective coupling constant in Kepler's third law) to gravitational radiation. In the limit $\zeta \rightarrow 0$, we recover GR, and all structure dependence disappears. The first term in $\dot{P}_{\mathrm{b}}$ (see Eq. (114)) is the combined effect of quadrupole and monopole gravitational radiation, post-Newtonian corrections to dipole radiation, and a dipole-octupole coupling term, all contributing at $0 \mathrm{PN}$ order, while the second term is the effect of dipole radiation, contributing at the dominant $-1 \mathrm{PN}$ order.

Unfortunately, because of the near equality of neutron star masses in typical double neutron star binary pulsars, dipole radiation is somewhat suppressed, and the bounds obtained are typically not competitive with the Cassini bound on $\gamma$, except for those generalized scalar-tensor theories, with $\beta_{0}<0$ where the strong gravity of the neutron stars induces spontaneous scalarization effects [106]. Figure 9 illustrates this: the bounds on $\alpha_{0}$ and $\beta_{0}$ from the three binary neutron star systems B1913+16, J0737-3039, and B1534+12 are not close to being competitive with the Cassini bound on $\alpha_{0}$, except for very negative values of $\beta_{0}$ (recall that $\left.\alpha_{0}=\left(3+2 \omega_{0}\right)^{-1 / 2}\right)$.

On the other hand, a binary pulsar system with dissimilar objects, such as a white dwarf or black hole companion, provides potentially more promising tests of dipole radiation. As a result, the neutron-star-white-dwarf systems J1141-6545 and J1738+0333 yield much more stringent bounds. 


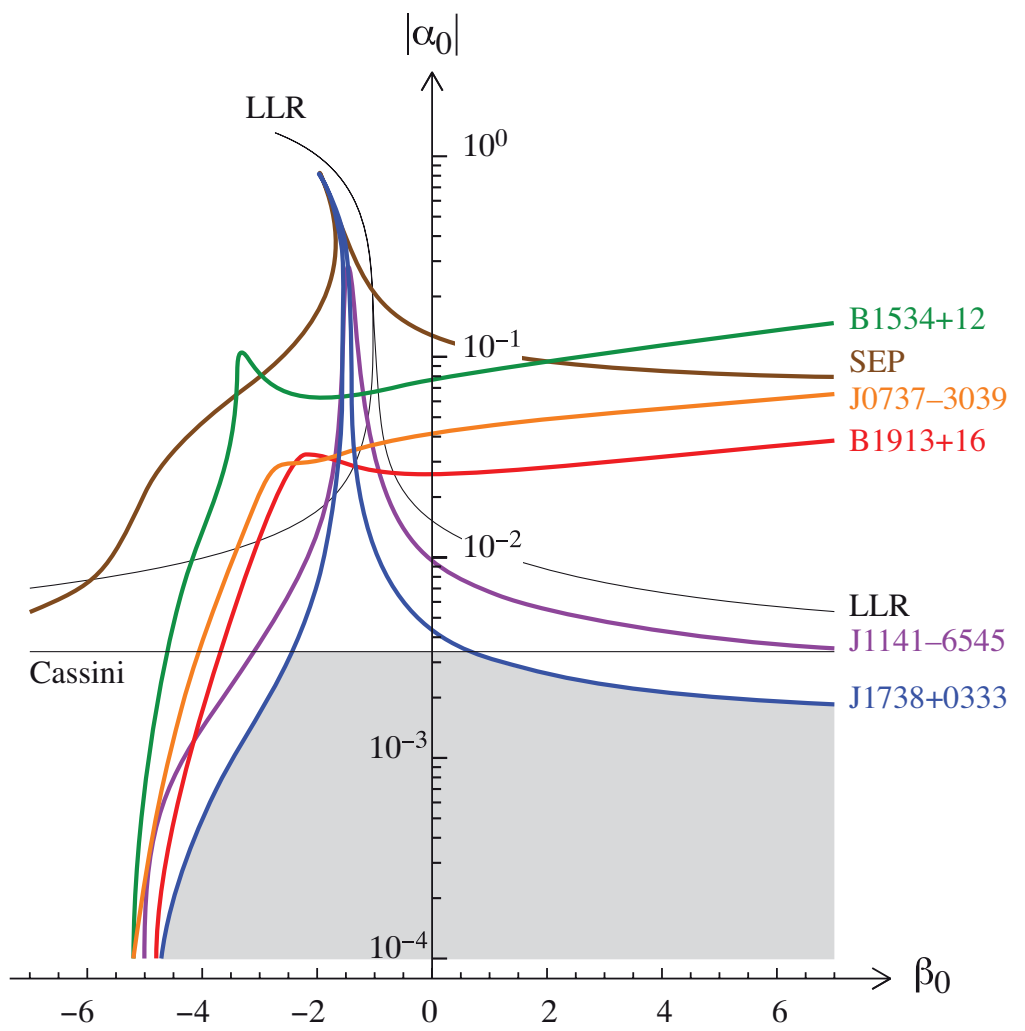

Figure 9: Bounds on the scalar-tensor parameters $\alpha_{0}$ and $\beta_{0}$ from solar-system and binary pulsar measurements. Bounds from tests of the Nordtvedt effect using lunar laser ranging and circular pulsar-white-dwarf binary systems are denoted LLR and SEP, respectively. Image reproduced with permission from [164], copyright by the authors.

Indeed, the latter system surpasses the Cassini bound for $\beta_{0}>1$ and $\beta_{0}<-2$, and is close to that bound for the pure Brans-Dicke case $\beta_{0}=0$ [164].

It is worth pointing out that the bounds displayed in Figure 9 have been calculated using a specific choice of scalar-tensor theory, in which the function $A(\varphi)$ is given by

$$
A(\varphi)=\exp \left[\alpha_{0}\left(\varphi-\varphi_{0}\right)+\frac{1}{2} \beta_{0}\left(\varphi-\varphi_{0}\right)^{2}\right],
$$

where $\alpha_{0}$, and $\beta_{0}$, are arbitrary parameters, and $\varphi_{0}$ is the asymptotic value of the scalar field. In the notation for scalar-tensor theories used here, this theory corresponds to the choice

$$
\omega(\phi)=-\frac{1}{2}\left(3-\frac{1}{\alpha_{0}^{2}-\beta_{0} \ln \phi}\right)
$$

where $\phi_{0}=A\left(\varphi_{0}\right)^{-2}=1$. The parameters $\zeta$ and $\lambda$ are given by

$$
\begin{aligned}
\zeta & =\frac{\alpha_{0}^{2}}{1+\alpha_{0}^{2}}, \\
\lambda & =\frac{1}{2} \frac{\beta_{0}}{1+\alpha_{0}^{2}} .
\end{aligned}
$$


The bounds were also calculated using a polytropic equation of state, which tends to give lower maximum masses for neutron stars than do more realistic equations of state.

Bounds on various versions of $\mathrm{TeVeS}$ theories have also been established, with the tightest constraints again coming from neutron-star-white-dwarf binaries [164]; in the case of TeVeS, the theory naturally predicts $\gamma=1$ in the post-Newtonian limit, so the Cassini measurements are irrelevant here. Strong constraints on the Einstein-Æther and Khronometric theories have also been placed using binary pulsar measurements, exploiting both gravitational-wave damping data, and data related to preferred-frame effects [443, 442]. 


\section{Gravitational-Wave Tests of Gravitational Theory}

\subsection{Gravitational-wave observatories}

Soon after the publication of this update, a new method of testing relativistic gravity will be realized, when a worldwide network of upgraded laser interferometric gravitational-wave observatories in the U.S. (LIGO Hanford and LIGO Livingston) and Europe (VIRGO and GEO600) begins regular detection and analysis of gravitational-wave signals from astrophysical sources. Within a few years, they may be joined by an underground cryogenic interferometer (KAGRA) in Japan, and around 2022, by a LIGO-type interferometer in India. These broad-band antennas will have the capability of detecting and measuring the gravitational waveforms from astronomical sources in a frequency band between about $10 \mathrm{~Hz}$ (the seismic noise cutoff) and $500 \mathrm{~Hz}$ (the photon counting noise cutoff), with a maximum sensitivity to strain at around $100 \mathrm{~Hz}$ of $h \sim \Delta l / l \sim 10^{-22}$ $(\mathrm{rms})$, for the kilometer-scale LIGO/VIRGO projects. The most promising source for detection and study of the gravitational wave signal is the "inspiralling compact binary" - a binary system of neutron stars or black holes (or one of each) in the final minutes of a death spiral leading to a violent merger. Such is the fate, for example, of the Hulse-Taylor binary pulsar B1913+16 in about 300 Myr, or the double pulsar J0737-3039 in about 85 Myr. Given the expected sensitivity of the advanced LIGO-Virgo detectors, which could see such sources out to many hundreds of megaparsecs, it has been estimated that from 40 to several hundred annual inspiral events could be detectable. Other sources, such as supernova core collapse events, instabilities in rapidly rotating newborn neutron stars, signals from non-axisymmetric pulsars, and a stochastic background of waves, may be detectable (see [352] for a review).

In addition, plans are being developed for orbiting laser interferometer space antennae, such as DECIGO in Japan and eLISA in Europe. The eLISA system would consist of three spacecraft orbiting the sun in a triangular formation separated from each other by a million kilometers, and would be sensitive primarily in the very low-frequency band between $10^{-4}$ and $10^{-1} \mathrm{~Hz}$, with peak strain sensitivity of order $h \sim 10^{-23}$.

A third approach that focuses on the ultra low-frequency band (nanohertz) is that of Pulsar Timing Arrays (PTA), whereby a network of highly stable millisecond pulsars is monitored in a coherent way using radio telescopes, in hopes of detecting the fluctuations in arrival times induced by passing gravitational waves.

For recent reviews of the status of all these approaches to gravitational-wave detection, see the Proceedings of the 8th Edoardo Amaldi Conference on Gravitational Waves [272].

In addition to opening a new astronomical window, the detailed observation of gravitational waves by such observatories may provide the means to test general relativistic predictions for the polarization and speed of the waves, for gravitational radiation damping and for strong-field gravity. These topics have been thoroughly covered in two recent Living Reviews by Gair et al. [170] for space-based detectors, and by Yunes and Siemens [452] for ground-based detectors. Here we present a brief overview.

\subsection{Gravitational-wave amplitude and polarization}

\subsubsection{General relativity}

A generic gravitational wave detector can be modelled as a body of mass $M$ at a distance $L$ from a fiducial laboratory point, connected to the point by a spring of resonant frequency $\omega_{0}$ and quality factor $Q$. From the equation of geodesic deviation, the infinitesimal displacement $\xi$ of the mass along the line of separation from its equilibrium position satisfies the equation of motion

$$
\ddot{\xi}+\frac{2 \omega_{0}}{Q} \dot{\xi}+\omega_{0}^{2} \xi=\frac{L}{2}\left(F_{+}(\theta, \phi, \psi) \ddot{h}_{+}(t)+F_{\times}(\theta, \phi, \psi) \ddot{h}_{\times}(t)\right)
$$


where $F_{+}(\theta, \phi, \psi)$ and $F_{\times}(\theta, \phi, \psi)$ are "beam-pattern" factors that depend on the direction of the source $(\theta, \phi)$ and on a polarization angle $\psi$, and $h_{+}(t)$ and $h_{\times}(t)$ are gravitational waveforms corresponding to the two polarizations of the gravitational wave (for pedagogical reviews, see [386, 324]). In a source coordinate system in which the $x-y$ plane is the plane of the sky and the $z$-direction points toward the detector, these two modes are given by

$$
h_{+}(t)=\frac{1}{2}\left(h_{\mathrm{TT}}^{x x}(t)-h_{\mathrm{TT}}^{y y}(t)\right), \quad h_{\times}(t)=h_{\mathrm{TT}}^{x y}(t),
$$

where $h_{\mathrm{TT}}^{i j}$ represent transverse-traceless (TT) projections of the calculated waveform of Eq. (84), given by

$$
h_{\mathrm{TT}}^{i j}=h^{k l}\left[\left(\delta^{i k}-\hat{N}^{i} \hat{N}^{k}\right)\left(\delta^{j l}-\hat{N}^{j} \hat{N}^{l}\right)-\frac{1}{2}\left(\delta^{i j}-\hat{N}^{i} \hat{N}^{j}\right)\left(\delta^{k l}-\hat{N}^{k} \hat{N}^{l}\right)\right],
$$

where $\hat{N}^{j}$ is a unit vector pointing toward the detector. The beam pattern factors depend on the orientation and nature of the detector. For a wave approaching along the laboratory $z$-direction, and for a mass whose location on the $x-y$ plane makes an angle $\phi$ with the $x$-axis, the beam pattern factors are given by $F_{+}=\cos 2 \phi$ and $F_{\times}=\sin 2 \phi$. For a laser interferometer with one arm along the laboratory $x$-axis, the other along the $y$-axis, with $\xi$ defined as the differential displacement along the two arms, the beam pattern functions are

$$
\begin{aligned}
& F_{+}=\frac{1}{2}\left(1+\cos ^{2} \theta\right) \cos 2 \phi \cos 2 \psi-\cos \theta \sin 2 \phi \sin 2 \psi, \\
& F_{\times}=\frac{1}{2}\left(1+\cos ^{2} \theta\right) \cos 2 \phi \sin 2 \psi+\cos \theta \sin 2 \phi \cos 2 \psi .
\end{aligned}
$$

Here, we assume that $\omega_{0} \approx 0$ in Eq. (124), corresponding to the essentially free motion of the suspended mirrors in the horizontal direction. For a laser interferometer in which the angle between the arms is $\chi$, the overall response is reduced by $\sin \chi$; for a space-based interferometer such as eLISA, $\chi=60^{\circ}$.

The waveforms $h_{+}(t)$ and $h_{\times}(t)$ depend on the nature and evolution of the source. For example, for a binary system in a circular orbit, with an inclination $i$ relative to the plane of the sky, and the $x$-axis oriented along the major axis of the projected orbit, the quadrupole approximation of Eq. (86) gives

$$
\begin{aligned}
& h_{+}(t)=-\frac{2 \mathcal{M}}{R}\left(2 \pi \mathcal{M} f_{\mathrm{b}}\right)^{2 / 3}\left(1+\cos ^{2} i\right) \cos 2 \Phi_{\mathrm{b}}(t), \\
& h_{\times}(t)=-\frac{2 \mathcal{M}}{R}\left(2 \pi \mathcal{M} f_{\mathrm{b}}\right)^{2 / 3}(2 \cos i) \cos 2 \Phi_{\mathrm{b}}(t),
\end{aligned}
$$

where $\Phi_{\mathrm{b}}(t)=2 \pi \int^{t} f_{\mathrm{b}}\left(t^{\prime}\right) d t^{\prime}$ is the orbital phase.

\subsubsection{Alternative theories of gravity}

A generic gravitational wave detector whose scale is small compared to the gravitational wavelength measures the local components of a symmetric $3 \times 3$ tensor which is composed of the "electric" components of the Riemann curvature tensor, $R_{0 i 0 j}$, via the equation of geodesic deviation, given, for a pair of freely falling particles by $\ddot{x}^{i}=-R_{0 i 0 j} x^{j}$, where $x^{i}$ denotes the spatial separation. In general there are six independent components, which can be expressed in terms of polarizations (modes with specific transformation properties under rotations and boosts); for a wave propagating in the $z$-direction, they can be displayed by the matrix

$$
S^{j k}=\left(\begin{array}{ccc}
A_{\mathrm{S}}+A_{+} & A_{\times} & A_{\mathrm{V} 1} \\
A_{\times} & A_{\mathrm{S}}-A_{+} & A_{\mathrm{V} 2} \\
A_{\mathrm{V} 1} & A_{\mathrm{V} 2} & A_{\mathrm{L}}
\end{array}\right) .
$$


Three modes $\left(A_{+}, A_{\times}\right.$, and $\left.A_{\mathrm{S}}\right)$ are transverse to the direction of propagation, with two representing quadrupolar deformations and one representing a monopolar transverse "breathing" deformation. Three modes are longitudinal, with one $\left(A_{\mathrm{L}}\right)$ an axially symmetric stretching mode in the propagation direction, and one quadrupolar mode in each of the two orthogonal planes containing the propagation direction $\left(A_{\mathrm{V} 1}\right.$ and $\left.A_{\mathrm{V} 2}\right)$. Figure 10 shows the displacements induced on a ring of freely falling test particles by each of these modes. General relativity predicts only the first two transverse quadrupolar modes (a) and (b) independently of the source; these correspond to the waveforms $h_{+}$and $h_{\times}$discussed earlier (note the $\cos 2 \phi$ and $\sin 2 \phi$ dependences of the displacements).

\section{Gravitational-Wave Polarization}
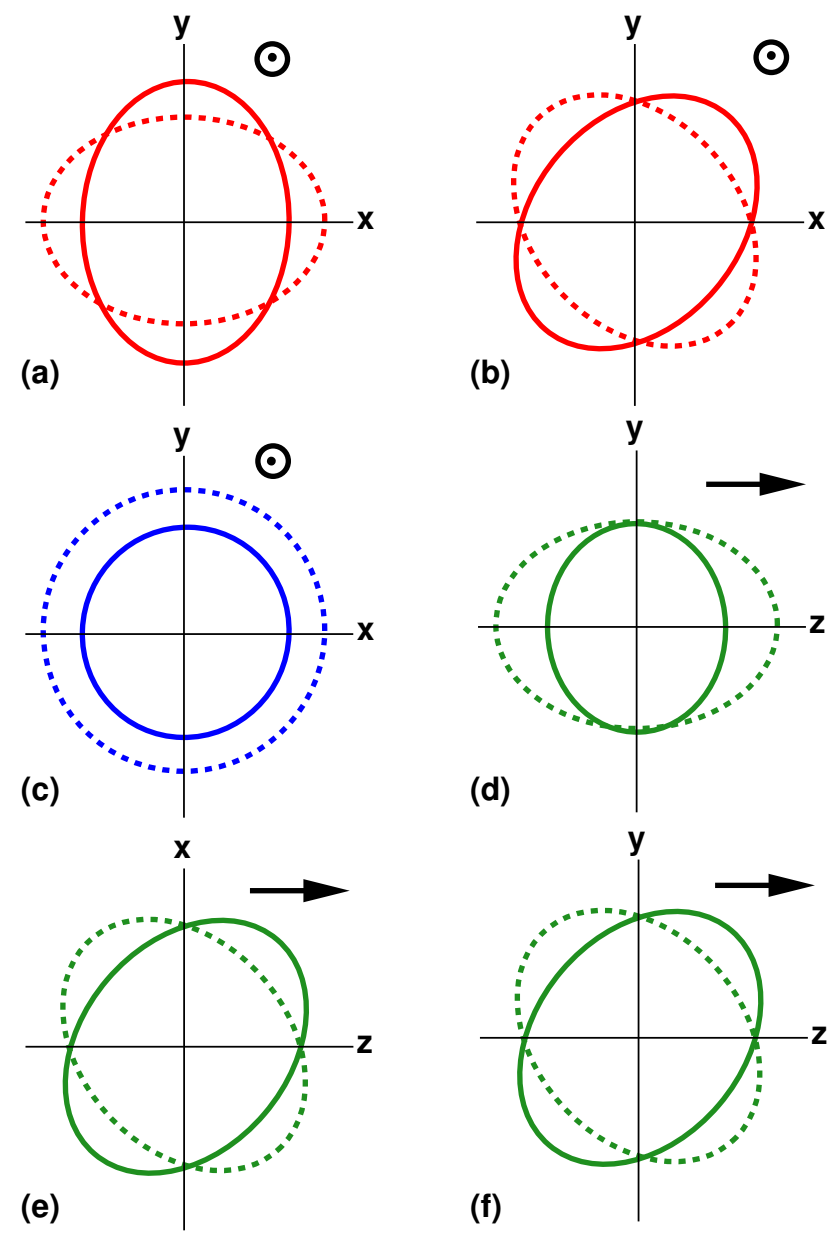

Figure 10: The six polarization modes for gravitational waves permitted in any metric theory of gravity. Shown is the displacement that each mode induces on a ring of test particles. The wave propagates in the $+z$ direction. There is no displacement out of the plane of the picture. In (a), (b), and (c), the wave propagates out of the plane; in (d), (e), and (f), the wave propagates in the plane. In GR, only (a) and (b) are present; in massless scalar-tensor gravity, (c) may also be present.

Massless scalar-tensor gravitational waves can in addition contain the transverse breathing mode (c). This can be obtained from the physical waveform $h^{\alpha \beta}$, which is related to $\tilde{h}^{\alpha \beta}$ and $\varphi$ to 
the required order by

$$
h^{\alpha \beta}=\tilde{h}^{\alpha \beta}+\Psi \eta^{\alpha \beta},
$$

where $\Psi=\varphi-1$. In this case, $A_{+(-)} \propto \tilde{h}_{+(-)}$, while $A_{\mathrm{S}} \propto \Psi$ (see Eqs. (99), (100), (101) and (102) for the leading contributions to these fields). In massive scalar-tensor theories, the longitudinal mode $(d)$ can also be present, but is suppressed relative to (c) by a factor $\left(\lambda / \lambda_{\mathrm{C}}\right)^{2}$, where $\lambda$ is the wavelength of the radiation, and $\lambda_{\mathrm{C}}$ is the Compton wavelength of the massive scalar.

More general metric theories predict additional longitudinal modes, up to the full complement of six (TEGP 10.2 [420]). For example, Einstein-Æther theory generically predicts all six modes [205].

A suitable array of gravitational antennas could delineate or limit the number of modes present in a given wave. The strategy depends on whether or not the source direction is known. In general there are eight unknowns (six polarizations and two direction cosines), but only six measurables $\left(R_{0 i 0 j}\right)$. If the direction can be established by either association of the waves with optical or other observations, or by time-of-flight measurements between separated detectors, then six suitably oriented detectors suffice to determine all six components. If the direction cannot be established, then the system is underdetermined, and no unique solution can be found. However, if one assumes that only transverse waves are present, then there are only three unknowns if the source direction is known, or five unknowns otherwise. Then the corresponding number (three or five) of detectors can determine the polarization. If distinct evidence were found of any mode other than the two transverse quadrupolar modes of GR, the result would be disastrous for GR. On the other hand, the absence of a breathing mode would not necessarily rule out scalar-tensor gravity, because the strength of that mode depends on the nature of the source.

For laser interferometers, the signal controlling the laser phase output can be written in the form

$$
S(t)=\frac{1}{2}\left(e_{1}^{j} e_{1}^{k}-e_{2}^{j} e_{2}^{k}\right) S^{j k},
$$

where $\mathbf{e}_{1}$ and $\mathbf{e}_{2}$ are unit vectors directed along the two arms of the interferometer. The final result is

$$
S(t)=F_{\mathrm{S}} A_{\mathrm{S}}+F_{\mathrm{L}} A_{\mathrm{L}}+F_{\mathrm{V} 1} A_{\mathrm{V} 1}+F_{\mathrm{V} 2} A_{\mathrm{V} 2}+F_{+} A_{+}+F_{\times} A_{\times},
$$

where the angular pattern functions $F_{A}(\theta, \phi, \psi)$ are given by

$$
\begin{aligned}
F_{\mathrm{S}} & =-\frac{1}{2} \sin ^{2} \theta \cos 2 \phi, \\
F_{\mathrm{L}} & =\frac{1}{2} \sin ^{2} \theta \cos 2 \phi, \\
F_{\mathrm{V} 1} & =-\sin \theta(\cos \theta \cos 2 \phi \cos \psi-\sin 2 \phi \sin \psi), \\
F_{\mathrm{V} 2} & =-\sin \theta(\cos \theta \cos 2 \phi \sin \psi+\sin 2 \phi \cos \psi), \\
F_{+} & =\frac{1}{2}\left(1+\cos ^{2} \theta\right) \cos 2 \phi \cos 2 \psi-\cos \theta \sin 2 \phi \sin 2 \psi, \\
F_{\times} & =\frac{1}{2}\left(1+\cos ^{2} \theta\right) \cos 2 \phi \sin 2 \psi+\cos \theta \sin 2 \phi \cos 2 \psi,
\end{aligned}
$$

(see [324] for detailed derivations and definitions). Note that the scalar and longitudinal pattern functions are degenerate and thus no array of laser interferometers can measure these two modes separately.

Some of the details of implementing such polarization observations have been worked out for arrays of resonant cylindrical, disk-shaped, spherical, and truncated icosahedral detectors (TEGP 10.2 [420], for recent reviews see [256, 403]). Early work to assess whether the ground-based or space-based laser interferometers (or combinations of the two types) could perform interesting polarization measurements was carried out in [404, 70, 267, 171, 411]; for a recent detailed analysis see [301]. Unfortunately for this purpose, the two LIGO observatories (in Washington and 
Louisiana states, respectively) have been constructed to have their respective arms as parallel as possible, apart from the curvature of the Earth; while this maximizes the joint sensitivity of the two detectors to gravitational waves, it minimizes their ability to detect two modes of polarization. In this regard the addition of Virgo, and the future KAGRA and LIGO-India systems will be crucial to polarization measurements. By combining signals from various interferometers into a kind of "null channel" one can test for the existence of modes beyond the + and $\times$ modes in a model independent manner [78]. The capability of space-based interferometers to measure the polarization modes was assessed in detail in [388, 302]. For pulsar timing arrays, see [245, 14, 74].

\subsection{Gravitational-wave phase evolution}

\subsubsection{General relativity}

In the binary pulsar, a test of GR was made possible by measuring at least three relativistic effects that depended upon only two unknown masses. The evolution of the orbital phase under the damping effect of gravitational radiation played a crucial role. Another situation in which measurement of orbital phase can lead to tests of GR is that of the inspiralling compact binary system. The key differences are that here gravitational radiation itself is the detected signal, rather than radio pulses, and the phase evolution alone carries all the information. In the binary pulsar, the first derivative of the binary frequency $\dot{f}_{\mathrm{b}}$ was measured; here the full nonlinear variation of $f_{\mathrm{b}}$ as a function of time is measured.

Broad-band laser interferometers are especially sensitive to the phase evolution of the gravitational waves, which carry the information about the orbital phase evolution. The analysis of gravitational wave data from such sources will involve some form of matched filtering of the noisy detector output against an ensemble of theoretical "template" waveforms which depend on the intrinsic parameters of the inspiralling binary, such as the component masses, spins, and so on, and on its inspiral evolution. How accurate must a template be in order to "match" the waveform from a given source (where by a match we mean maximizing the cross-correlation or the signal-tonoise ratio)? In the total accumulated phase of the wave detected in the sensitive bandwidth, the template must match the signal to a fraction of a cycle. For two inspiralling neutron stars detected by the advanced LIGO/Virgo systems, around 16000 cycles should be detected during the final few minutes of inspiral; this implies a phasing accuracy of $10^{-5}$ or better. Since $v \sim 1 / 10$ during the late inspiral, this means that correction terms in the phasing at the level of $v^{5}$ or higher are needed. More formal analyses confirm this intuition [99, 153, 97, 323].

Because it is a slow-motion system $\left(v \sim 10^{-3}\right)$, the binary pulsar is sensitive only to the lowestorder effects of gravitational radiation as predicted by the quadrupole formula. Nevertheless, the first correction terms of order $v$ and $v^{2}$ to the quadrupole formula were calculated as early as 1976 [405] (see TEGP 10.3 [420]).

But for laser interferometric observations of gravitational waves, the bottom line is that, in order to measure the astrophysical parameters of the source and to test the properties of the gravitational waves, it is necessary to derive the gravitational waveform and the resulting radiation back-reaction on the orbit phasing at least to $3 \mathrm{PN}$ order beyond the quadrupole approximation.

For the special case of non-spinning bodies moving on quasi-circular orbits (i.e., circular apart from a slow inspiral), the evolution of the gravitational wave frequency $f=2 f_{\mathrm{b}}$ through $2 \mathrm{PN}$ order has the form

$$
\begin{aligned}
\dot{f}=\frac{96 \pi}{5} f^{2}(\pi \mathcal{M} f)^{5 / 3}[ & 1-\left(\frac{743}{336}+\frac{11}{4} \eta\right)(\pi m f)^{2 / 3}+4 \pi(\pi m f) \\
& \left.+\left(\frac{34103}{18144}+\frac{13661}{2016} \eta+\frac{59}{18} \eta^{2}\right)(\pi m f)^{4 / 3}+\mathcal{O}\left[(\pi m f)^{5 / 3}\right]\right]
\end{aligned}
$$


where $\eta=m_{1} m_{2} / m^{2}$. The first term is the quadrupole contribution (compare Eq. (88)), the second term is the 1PN contribution, the third term, with the coefficient $4 \pi$, is the "tail" contribution, and the fourth term is the $2 \mathrm{PN}$ contribution. Two decades of intensive work by many groups have led to the development of waveforms in GR that are accurate to 3.5PN order, and for some specific effects, such as those related to spin, to 4.5PN order (see [51] for a thorough review).

Similar expressions can be derived for the loss of angular momentum and linear momentum. Expressions for non-circular orbits have also been derived [175, 107]. These losses react back on the orbit to circularize it and cause it to inspiral. The result is that the orbital phase (and consequently the gravitational wave phase) evolves non-linearly with time. It is the sensitivity of the broad-band laser interferometric detectors to phase that makes the higher-order contributions to $d f / d t$ so observationally relevant.

If the coefficients of each of the powers of $f$ in Eq. (135) can be measured, then one again obtains more than two constraints on the two unknowns $m_{1}$ and $m_{2}$, leading to the possibility to test GR. For example, Blanchet and Sathyaprakash [59,60] have shown that, by observing a source with a sufficiently strong signal, an interesting test of the $4 \pi$ coefficient of the "tail" term could be performed (but see [22] for a more sophisticated analysis).

Another possibility involves gravitational waves from a small mass orbiting and inspiralling into a (possibly supermassive) spinning black hole. A general non-circular, non-equatorial orbit will precess around the hole, both in periastron and in orbital plane, leading to a complex gravitational waveform that carries information about the non-spherical, strong-field spacetime around the hole. According to GR, this spacetime must be the Kerr spacetime of a rotating black hole, uniquely specified by its mass and angular momentum, and consequently, observation of the waves could test this fundamental hypothesis of GR [345, 322].

\subsubsection{Alternative theories of gravity}

In general, alternative theories of gravity will predict rather different phase evolution from that of GR, notably via the addition of dipole gravitational radiation. For example, the dipole gravitational radiation predicted by scalar-tensor theories modifies the gravitational radiation back-reaction, and thereby the phase evolution. Including only the leading $0 \mathrm{PN}$ and $-1 \mathrm{PN}$ (dipole) contributions, one obtains,

$$
\dot{f}=\frac{96 \pi}{5} f^{2}(\pi \alpha \mathcal{M} f)^{5 / 3} \frac{\kappa_{1}}{12}\left[1+b(\pi m f)^{-2 / 3}\right],
$$

where $\mathcal{M}=\eta^{3 / 5} m$, and $b$ is the coefficient of the dipole term, given to first order in $\zeta$ by $b=$ $(5 / 24) \zeta \alpha^{-5 / 3} \mathcal{S}^{2}$, where $\kappa_{1}$ is given by Eq. (104), $\mathcal{S}=\alpha^{-1 / 2}\left(s_{1}-s_{2}\right)$ and $\zeta=1 /\left(4+2 \omega_{0}\right)$. Double neutron star systems are not promising because the small range of masses available near $1.4 M_{\odot}$ results in suppression of dipole radiation by symmetry. For black holes, $s=0.5$ identically, consequently double black hole systems turn out to be observationally identical in the two theories. Thus mixed systems involving a neutron star and a black hole are preferred. However, a number of analyses of the capabilities of both ground-based and space-based (eLISA) observatories have shown that observing waves from neutron-star-black-hole inspirals is not likely to bound scalar-tensor gravity at a level competitive with the Cassini bound, with future solar-system improvements, or with binary pulsar observations [422, 236, 106, 353, 433, 41, 42, 445]. A possible exception is DECIGO/BBO, a proposed space gravitational-wave observatory with peak sensitivity between the eLISA and LIGO/Virgo bands; observations of inspirals of neutron stars onto hypothetical intermediate mass $\left(\sim 10^{4} M_{\odot}\right)$ black holes could improve upon the Cassini bound by several orders of magnitude [446].

These considerations suggest that it might be fruitful to attempt to parametrize the phasing formulae in a manner reminiscent of the PPN framework for post-Newtonian gravity. A number of approaches along this line have been developed, including the parametrized post-Einsteinian (PPE) 
framework [451, 347], a Bayesian parametrized approach [250], and a parametrization based on the post-Newtonian expansions discussed above [288]. The discovery of relationships between the moment of inertia, the gravitational Love number, and the quadrupole moment of neutron stars ("I-Love-Q" relations) in general relativity has opened the possibility of testing theories using gravitational waves in a manner that is relatively free of contamination from the neutron-star equation of state [448, 447].

\subsection{Speed of gravitational waves}

According to GR, in the limit in which the wavelength of gravitational waves is small compared to the radius of curvature of the background spacetime, the waves propagate along null geodesics of the background spacetime, i.e., they have the same speed $c$ as light (in this section, we do not set $c=1$ ). In other theories, the speed could differ from $c$ because of coupling of gravitation to "background" gravitational fields. For example, in the Rosen bimetric theory with a flat background metric $\boldsymbol{\eta}$, gravitational waves follow null geodesics of $\boldsymbol{\eta}$, while light follows null geodesics of $\mathbf{g}$ (TEGP $10.1[420]$ ).

Another way in which the speed of gravitational waves could differ from $c$ is if gravitation were propagated by a massive field (a massive graviton), in which case $v_{\mathrm{g}}$ would be given by, in a local inertial frame,

$$
\frac{v_{\mathrm{g}}^{2}}{c^{2}}=1-\frac{m_{\mathrm{g}}^{2} c^{4}}{E^{2}}
$$

where $m_{\mathrm{g}}$ and $E$ are the graviton rest mass and energy, respectively.

The most obvious way to test this is to compare the arrival times of a gravitational wave and an electromagnetic wave from the same event, e.g., a supernova or a prompt gamma-ray burst. For a source at a distance $D$, the resulting value of the difference $1-v_{\mathrm{g}} / c$ is

$$
1-\frac{v_{\mathrm{g}}}{c}=5 \times 10^{-17}\left(\frac{200 \mathrm{Mpc}}{D}\right)\left(\frac{\Delta t}{1 \mathrm{~s}}\right),
$$

where $\Delta t \equiv \Delta t_{\mathrm{a}}-(1+Z) \Delta t_{\mathrm{e}}$ is the "time difference", where $\Delta t_{\mathrm{a}}$ and $\Delta t_{\mathrm{e}}$ are the differences in arrival time and emission time of the two signals, respectively, and $Z$ is the redshift of the source. In many cases, $\Delta t_{\mathrm{e}}$ is unknown, so that the best one can do is employ an upper bound on $\Delta t_{\mathrm{e}}$ based on observation or modelling. The result will then be a bound on $1-v_{\mathrm{g}} / c$.

For a massive graviton, if the frequency of the gravitational waves is such that $h f \gg m_{\mathrm{g}} c^{2}$, where $h$ is Planck's constant, then $v_{\mathrm{g}} / c \approx 1-\frac{1}{2}\left(c / \lambda_{\mathrm{g}} f\right)^{2}$, where $\lambda_{\mathrm{g}}=h / m_{\mathrm{g}} c$ is the graviton Compton wavelength, and the bound on $1-v_{\mathrm{g}} / c$ can be converted to a bound on $\lambda_{\mathrm{g}}$, given by

$$
\lambda_{\mathrm{g}}>3 \times 10^{12} \mathrm{~km}\left(\frac{D}{200 \mathrm{Mpc}} \frac{100 \mathrm{~Hz}}{f}\right)^{1 / 2}\left(\frac{1}{f \Delta t}\right)^{1 / 2}
$$

The foregoing discussion assumes that the source emits both gravitational and electromagnetic radiation in detectable amounts, and that the relative time of emission can be established to sufficient accuracy, or can be shown to be sufficiently small.

However, there is a situation in which a bound on the graviton mass can be set using gravitational radiation alone [423]. That is the case of the inspiralling compact binary. Because the frequency of the gravitational radiation sweeps from low frequency at the initial moment of observation to higher frequency at the final moment, the speed of the gravitons emitted will vary, from lower speeds initially to higher speeds (closer to $c$ ) at the end. This will cause a distortion of the observed phasing of the waves and result in a shorter than expected overall time $\Delta t_{\mathrm{a}}$ of passage of a given number of cycles. Furthermore, through the technique of matched filtering, the parameters of the compact binary can be measured accurately (assuming that GR is a good approximation to 
the orbital evolution, even in the presence of a massive graviton), and thereby the emission time $\Delta t_{\mathrm{e}}$ can be determined accurately. Roughly speaking, the "phase interval" $f \Delta t$ in Eq. (139) can be measured to an accuracy $1 / \rho$, where $\rho$ is the signal-to-noise ratio.

Thus one can estimate the bounds on $\lambda_{\mathrm{g}}$ achievable for various compact inspiral systems, and for various detectors. For stellar-mass inspiral (neutron stars or black holes) observed by the LIGO/VIRGO class of ground-based interferometers, $D \approx 200 \mathrm{Mpc}, f \approx 100 \mathrm{~Hz}$, and $f \Delta t \sim$ $\rho^{-1} \approx 1 / 10$. The result is $\lambda_{\mathrm{g}}>10^{13} \mathrm{~km}$. For supermassive binary black holes $\left(10^{4}\right.$ to $\left.10^{7} M_{\odot}\right)$ observed by the proposed laser interferometer space antenna (LISA), $D \approx 3 \mathrm{Gpc}, f \approx 10^{-3} \mathrm{~Hz}$, and $f \Delta t \sim \rho^{-1} \approx 1 / 1000$. The result is $\lambda_{\mathrm{g}}>10^{17} \mathrm{~km}$.

A full noise analysis using proposed noise curves for the advanced LIGO and for LISA weakens these crude bounds by factors between two and 10 [423, 433, 41, 42, 23, 377, 445]. For example, for the inspiral of two $10^{6} M_{\odot}$ black holes with aligned spins at a distance of $3 \mathrm{Gpc}$ observed by LISA, a bound of $2 \times 10^{16} \mathrm{~km}$ could be placed [41]. Other possibilities include using binary pulsar data to bound modifications of gravitational radiation damping by a massive graviton [154], using LISA observations of the phasing of waves from compact white-dwarf binaries, eccentric galactic binaries, and eccentric inspiral binaries [98, 209], using pulsar timing arrays [244], and using DECIGO/BBO to observe neutron-star intermediate-mass black-hole inspirals [446].

Bounds obtainable from gravitational radiation effects should be compared with the solid bound $\lambda_{\mathrm{g}}>2.8 \times 10^{12} \mathrm{~km}$ [381] derived from solar system dynamics, which limit the presence of a Yukawa modification of Newtonian gravity of the form

$$
V(r)=\frac{G M}{r} \exp \left(-r / \lambda_{\mathrm{g}}\right),
$$

and with the model-dependent bound $\lambda_{\mathrm{g}}>6 \times 10^{19} \mathrm{~km}$ from consideration of galactic and cluster dynamics [401].

Mirshekari et al. [287] studied bounds that could be placed on more general graviton dispersion relations that could emerge from alternative theories with Lorentz violation, in which the effective propagation speed is given by

$$
\frac{v_{g}^{2}}{c^{2}}=1-\frac{m_{\mathrm{g}}^{2} c^{4}}{E^{2}}-\mathbb{A} E^{\alpha-2},
$$

where $\mathbb{A}$ and $\alpha$ are parameters that depend on the theory. 


\section{Astrophysical and Cosmological Tests}

One of the central difficulties of testing GR in the strong-field regime is the possibility of contamination by uncertain or complex physics. In the solar system, weak-field gravitational effects can in most cases be measured cleanly and separately from non-gravitational effects. The remarkable cleanliness of many binary pulsars permits precise measurements of gravitational phenomena in a strong-field context.

Unfortunately, nature is rarely so kind. Still, under suitable conditions, qualitative and even quantitative strong-field tests of GR could be carried out.

One example is the exploration of the spacetime near black holes and neutron stars. Studies of certain kinds of accretion known as advection-dominated accretion flow (ADAF) in low-luminosity binary X-ray sources may yield the signature of the black hole event horizon [299]. The spectrum of frequencies of quasi-periodic oscillations (QPO) from galactic black hole binaries may permit measurement of the spins of the black holes [327]. Aspects of strong-field gravity and framedragging may be revealed in spectral shapes of iron fluorescence lines from the inner regions of accretion disks [336, 337]. Using sub-mm VLBI, a collaboration dubbed the Event Horizon Telescope could image our galactic center black hole Sgr A* and the black hole in M87 with horizon-scale angular resolution; observation of accretion phenomena at these angular resolutions could provide tests of the spacetime geometry very close to the black hole [133]. Tracking of hypothetical stars whose orbits are within a fraction of a milliparsec of Sgr $\mathrm{A}^{*}$ could test the black hole "ho-hair" theorem, via a direct measurement of both the angular momentum $J$ and quadrupole moment $Q$ of the black hole, and a test of the requirement that $Q=-J^{2} / M$ [427]. Such tests could also be carried out using pulsars, if any should be found deep in the galactic center [255].

Because of uncertainties in the detailed models, the results to date of studies like these are suggestive at best, but the combination of future higher-resolution observations and better modelling could lead to striking tests of strong-field predictions of GR.

For a detailed review of strong-field tests of GR using electromagnetic observations, see [328].

Another example is in cosmology. From a few seconds after the Big Bang until the present, the underlying physics of the universe is well understood, in terms of a standard model of a nearly spatially flat universe, 13.6 Gyr old, dominated by cold dark matter and dark energy $(\Lambda \mathrm{CDM})$. Some alternative theories of gravity that are qualitatively different from GR fail to produce cosmologies that meet even the minimum requirements of agreeing qualitatively with Big-Bang nucleosynthesis (BBN) or the properties of the cosmic microwave background (TEGP 13.2 [420]). Others, such as Brans-Dicke theory, are sufficiently close to GR (for large enough $\omega_{\mathrm{BD}}$ ) that they conform to all cosmological observations, given the underlying uncertainties. The generalized scalar-tensor theories and $f(R)$ theories, however, could have small effective $\omega$ at early times, while evolving through the attractor mechanism to large $\omega$ today.

One way to test such theories is through Big-Bang nucleosynthesis, since the abundances of the light elements produced when the temperature of the universe was about $1 \mathrm{MeV}$ are sensitive to the rate of expansion at that epoch, which in turn depends on the strength of interaction between geometry and the scalar field. Because the universe is radiation-dominated at that epoch, uncertainties in the amount of cold dark matter or of the cosmological constant are unimportant. The nuclear reaction rates are reasonably well understood from laboratory experiments and theory, and the number of light neutrino families (3) conforms to evidence from particle accelerators. Thus, within modest uncertainties, one can assess the quantitative difference between the BBN predictions of GR and scalar-tensor gravity under strong-field conditions and compare with observations. For recent analyses, see [350, 116, 89, 90].

In addition, many alternative theories, such as $f(R)$ theories have been developed in order to provide an alternative to the dark energy of the standard $\Lambda \mathrm{CDM}$ model, in particular by 
modifying gravity on large, cosmological scales, while preserving the conventional solar and stellar system phenomenology of GR. Since we are now in a period of what may be called "precision cosmology", one can begin to envision trying to test alternative theories using the accumulation of data on many fronts, including the growth of large scale structure, cosmic background fluctuations, galactic rotation curves, BBN, weak lensing, baryon acoustic oscillations, etc. The "parametrized post-Friedmann" framework is one initial foray into this arena [30]. Other approaches can be found in $[15,121,135,134,454,189]$.

\section{Conclusions}

General relativity has held up under extensive experimental scrutiny. The question then arises, why bother to continue to test it? One reason is that gravity is a fundamental interaction of nature, and as such requires the most solid empirical underpinning we can provide. Another is that all attempts to quantize gravity and to unify it with the other forces suggest that the standard general relativity of Einstein may not be the last word. Furthermore, the predictions of general relativity are fixed; the pure theory contains no adjustable constants so nothing can be changed. Thus every test of the theory is either a potentially deadly test or a possible probe for new physics. Although it is remarkable that this theory, born 100 years ago out of almost pure thought, has managed to survive every test, the possibility of finding a discrepancy will continue to drive experiments for years to come. These experiments will search for new physics beyond Einstein at many different scales: the large distance scales of the astrophysical, galactic, and cosmological realms; scales of very short distances or high energy; and scales related to strong or dynamical gravity. 


\section{Acknowledgments}

This work has been supported since the initial version in part by the National Science Foundation, Grant Numbers PHY 96-00049, 00-96522, 03-53180, 06-52448, 09-65133, 12-60995 and 13-06069, and by the National Aeronautics and Space Administration, Grant Numbers NAG510186 and NNG-06GI60G. We also gratefully acknowledge the continuing hospitality of the Institut d'Astrophysique de Paris, where portions of this update were completed. We thank Luc Blanchet for helpful comments, and Michael Kramer and Norbert Wex for providing useful figures. We are particularly grateful to Norbert Wex and Nicolás Yunes for detailed and comprehensive comments. 


\section{References}

[1] Adelberger, E. G., "New tests of Einstein's equivalence principle and Newton's inverse-square law", Class. Quantum Grav., 18, 2397-2405 (2001). [DOI], [ADS]. (Cited on pages 11 and 48.)

[2] Adelberger, E. G., Heckel, B. R., Hoedl, S., Hoyle, C. D., Kapner, D. J. and Upadhye, A., "ParticlePhysics Implications of a Recent Test of the Gravitational Inverse-Square Law", Phys. Rev. Lett., 98, 131104 (2007). [DOI], [ADS], [hep-ph/0611223]. (Cited on page 27.)

[3] Adelberger, E. G., Heckel, B. R. and Nelson, A. E., "Tests of the Gravitational Inverse-Square Law", Annu. Rev. Nucl. Part. Sci., 53, 77-121 (2003). [DOI], [ADS], [arXiv:hep-ph/0307284 [hep-ph]]. (Cited on page 27.)

[4] Adelberger, E. G., Heckel, B. R., Stubbs, C. W. and Rogers, W. F., "Searches for New Macroscopic Forces", Annu. Rev. Nucl. Sci., 41, 269-320 (1991). [DOI]. (Cited on page 27.)

[5] Alexander, S. and Yunes, N., "Chern-Simons modified general relativity", Phys. Rep., 480, 1-55 (2009). [DOI], [ADS], [arXiv:0907.2562 [hep-th]]. (Cited on page 41.)

[6] Ali-Haïmoud, Y. and Chen, Y., "Slowly rotating stars and black holes in dynamical Chern-Simons gravity", Phys. Rev. D, 84, 124033 (2011). [DOI], [ADS], [arXiv:1110.5329 [astro-ph.HE]]. (Cited on page 41.)

[7] Alsing, J., Berti, E., Will, C. M. and Zaglauer, H., "Gravitational radiation from compact binary systems in the massive Brans-Dicke theory of gravity", Phys. Rev. D, 85, 064041 (2012). [DOI], [ADS], [arXiv:1112.4903 [gr-qc]]. (Cited on pages 62 and 66.)

[8] Alväger, T., Farley, F. J. M., Kjellman, J. and Wallin, I., "Test of the second postulate of special relativity in the GeV region", Phys. Lett., 12, 260-262 (1977). [DOI], [ADS]. (Cited on page 12.)

[9] Alvarez, C. and Mann, R. B., "The equivalence principle and anomalous magnetic moment experiments", Phys. Rev. D, 54, 7097-7107 (1996). [DOI], [gr-qc/9511028]. (Cited on page 22.)

[10] Alvarez, C. and Mann, R. B., "Testing the equivalence principle by Lamb shift energies", Phys. Rev. $D, \mathbf{5 4}, 5954-5974$ (1996). [DOI], [gr-qc/9507040]. (Cited on page 22.)

[11] Alvarez, C. and Mann, R. B., "The equivalence principle and $g-2$ experiments", Phys. Lett. B, 409, 83-87 (1997). [DOI], [gr-qc/9510070]. (Cited on page 22.)

[12] Alvarez, C. and Mann, R. B., "The equivalence principle in the non-baryonic regime", Phys. Rev. $D, \mathbf{5 5}, 1732-1740$ (1997). [DOI], [gr-qc/9609039]. (Cited on page 22.)

[13] Alvarez, C. and Mann, R. B., "Testing the equivalence principle using atomic vacuum energy shifts", Mod. Phys. Lett. A, 11, 1757-1763 (1997). [gr-qc/9612031]. (Cited on page 22.)

[14] Alves, M. E. S. and Tinto, M., "Pulsar timing sensitivities to gravitational waves from relativistic metric theories of gravity", Phys. Rev. D, 83, 123529 (2011). [DOI], [ADS], [arXiv:1102.4824 [gr-qc]]. (Cited on page 83.)

[15] Amin, M. A., Wagoner, R. V. and Blandford, R. D., "A subhorizon framework for probing the relationship between the cosmological matter distribution and metric perturbations", Mon. Not. R. Astron. Soc., 390, 131-142 (2008). [DOI], [ADS], [arXiv:0708.1793]. (Cited on page 88.)

[16] Anderson, J. D., Laing, P. A., Lau, E. L., Liu, A. S., Nieto, M. M. and Turyshev, S. G., "Indication, from Pioneer 10/11, Galileo, and Ulysses Data, of an Apparent Anomalous, Weak, Long-Range Acceleration", Phys. Rev. Lett., 81, 2858-2861 (1998). [DOI], [ADS], [gr-qc/9808081]. (Cited on page 27.) 
[17] Antia, H. M., Chitre, S. M. and Gough, D. O., "Temporal variations in the Sun's rotational kinetic energy", Astron. Astrophys., 477, 657-663 (2008). [DOI], [ADS], [arXiv:0711.0799]. (Cited on page 47.$)$

[18] Antoniadis, I., Arkani-Hamed, N., Dimopoulos, S. and Dvali, G., "New dimensions at a millimeter to a fermi and superstrings at a TeV", Phys. Lett. B, 436, 257-263 (1998). [DOI], [hep-ph/9804398]. (Cited on page 27.)

[19] Antoniadis, J. et al., "A Massive Pulsar in a Compact Relativistic Binary", Science, 340, 448 (2013). [DOI], [ADS], [arXiv:1304.6875 [astro-ph.HE]]. (Cited on page 73.)

[20] Antonini, P., Okhapkin, M., Göklü, E. and Schiller, S., "Test of constancy of speed of light with rotating cryogenic optical resonators", Phys. Rev. A, 71, 050101 (2005). [gr-qc/0504109]. (Cited on page 13.)

[21] Arkani-Hamed, N., Dimopoulos, S. and Dvali, G. R., "The hierarchy problem and new dimensions at a millimeter", Phys. Lett. B, 429, 263-272 (1998). [DOI], [arXiv:hep-ph/9803315]. (Cited on page 27.)

[22] Arun, K. G., Iyer, B. R., Qusailah, M. S. S. and Sathyaprakash, B. S., "Probing the nonlinear structure of general relativity with black hole binaries", Phys. Rev. D, 74, 024006 (2006). [DOI], [ADS], [gr-qc/0604067]. (Cited on page 84.)

[23] Arun, K. G. and Will, C. M., "Bounding the mass of the graviton with gravitational waves: effect of higher harmonics in gravitational waveform templates", Class. Quantum Grav., 26, 155002 (2009). [DOI], [ADS], [arXiv:0904.1190 [gr-qc]]. (Cited on page 86.)

[24] Asada, H., "The light cone effect on the Shapiro time delay", Astrophys. J. Lett., 574, L69-L70 (2002). [DOI], [astro-ph/0206266]. (Cited on page 45.)

[25] Ashby, N., "Relativistic effects in the Global Positioning System", in Dadhich, N. and Narlikar, J. V., eds., Gravitation and Relativity: At the Turn of the Millenium, Proceedings of the 15th International Conference on General Relativity and Gravitation (GR-15), held at IUCAA, Pune, India, December 16-21, 1997, pp. 231-258, (Inter-University Center for Astronomy and Astrophysics, Pune, India, 1998). (Cited on page 17.)

[26] Ashby, N., "Relativity in the Global Positioning System", Living Rev. Relativity, 6, lrr-2003-1 (2003). [DOI]. URL (accessed 28 March 2014):

http://www.livingreviews.org/lrr-2003-1. (Cited on page 17.)

[27] Ashby, N., Bender, P. L. and Wahr, J. M., "Future gravitational physics tests from ranging to the BepiColombo Mercury planetary orbiter", Phys. Rev. D, 75, 022001 (2007). [DOI], [ADS]. (Cited on page 54.)

[28] "ATNF Pulsar Catalogue", web interface to database, Australia Telescope National Facility. URL (accessed 28 March 2014): http://www.atnf.csiro.au/research/pulsar/psrcat/. (Cited on page 68.)

[29] Baeßler, S., Heckel, B. R., Adelberger, E. G., Gundlach, J. H., Schmidt, U. and Swanson, H. E., "Improved Test of the Equivalence Principle for Gravitational Self-Energy", Phys. Rev. Lett., 83, 3585-3588 (1999). [DOI], [ADS]. (Cited on pages 11 and 48.)

[30] Baker, T., Ferreira, P. G. and Skordis, C., "The parameterized post-Friedmann framework for theories of modified gravity: Concepts, formalism, and examples", Phys. Rev. D, 87, 024015 (2013). [DOI], [ADS], [arXiv:1209.2117 [astro-ph.CO]]. (Cited on page 88.)

[31] Bambi, C., Giannotti, M. and Villante, F. L., "Response of primordial abundances to a general modification of $G_{\mathrm{N}}$ and/or of the early universe expansion rate", Phys. Rev. D, 71, 123524 (2005). [DOI], [astro-ph/0503502]. (Cited on page 50.) 
[32] Barausse, E., Palenzuela, C., Ponce, M. and Lehner, L., "Neutron-star mergers in scalar-tensor theories of gravity", Phys. Rev. D, 87, 081506 (2013). [DOI], [ADS], [arXiv:1212.5053 [gr-qc]]. (Cited on page 37.$)$

[33] Bartlett, D. F. and Van Buren, D., "Equivalence of Active and Passive Gravitational Mass Using the Moon", Phys. Rev. Lett., 57, 21-24 (1986). [DOI]. (Cited on page 53.)

[34] Bauch, A. and Weyers, S., "New experimental limit on the validity of local position invariance", Phys. Rev. D, 65, 081101(R) (2002). [DOI], [ADS]. (Cited on page 17.)

[35] Baumgarte, T. W. and Shapiro, S. L., Numerical Relativity: Solving Einstein's Equations on the Computer, (Cambridge University Press, Cambridge; New York, 2010). [ADS], [Google Books]. (Cited on page 55.)

[36] Bekenstein, J. D., "Relativistic gravitation theory for the modified Newtonian dynamics paradigm", Phys. Rev. D, 70, 083509 (2004). [DOI], [ADS], [arXiv:astro-ph/0403694]. (Cited on page 39.)

[37] Bell, J. F. and Damour, T., "A new test of conservation laws and Lorentz invariance in relativistic gravity", Class. Quantum Grav., 13, 3121-3127 (1996). [DOI], [gr-qc/9606062]. (Cited on page 49.)

[38] Benkhoff, J. et al., "BepiColombo - Comprehensive exploration of Mercury: Mission overview and science goals", Planet. Space Sci., 58, 2-20 (2010). [DOI], [ADS]. (Cited on page 54.)

[39] Bennett, C. L. et al., "Nine-year Wilkinson Microwave Anisotropy Probe (WMAP) observations: Final maps and results", Astrophys. J. Suppl. Ser., 208, 20 (2013). [DOI], [ADS], [arXiv:1212.5225 [astro-ph.CO]]. (Cited on page 50.)

[40] Beringer, J. et al. (Particle Data Group), "Review of Particle Physics", Phys. Rev. D, 86, 010001 (2012). [DOI]. (Cited on page 8.)

[41] Berti, E., Buonanno, A. and Will, C. M., "Estimating spinning binary parameters and testing alternative theories of gravity with LISA", Phys. Rev. D, 71, 084025 (2005). [DOI], [ADS], [arXiv:grqc/0411129]. (Cited on pages 84 and 86.)

[42] Berti, E., Buonanno, A. and Will, C. M., "Testing general relativity and probing the merger history of massive black holes with LISA", Class. Quantum Grav., 22, S943-S954 (2005). [DOI], [ADS], [arXiv:gr-qc/0504017]. (Cited on pages 84 and 86.)

[43] Berti, E., Cardoso, V. and Starinets, A. O., "Quasinormal modes of black holes and black branes", Class. Quantum Grav., 26, 163001 (2009). [DOI], [ADS], [arXiv:0905.2975 [gr-qc]]. (Cited on page 55.)

[44] Bertotti, B., Iess, L. and Tortora, P., "A test of general relativity using radio links with the Cassini spacecraft", Nature, 425, 374-376 (2003). [DOI], [ADS]. (Cited on page 45.)

[45] Bezerra, V. B., Klimchitskaya, G. L., Mostepanenko, V. M. and Romero, C., "Constraints on nonNewtonian gravity from measuring the Casimir force in a configuration with nanoscale rectangular corrugations", Phys. Rev. D, 83, 075004 (2011). [DOI], [ADS], [arXiv:1103.0993 [hep-ph]]. (Cited on page 27.)

[46] Bhat, N. D. R., Bailes, M. and Verbiest, J. P. W., "Gravitational-radiation losses from the pulsar white-dwarf binary PSR J1141 6545", Phys. Rev. D, 77, 124017 (2008). [DOI], [ADS], [arXiv:0804.0956]. (Cited on pages 73 and 75.)

[47] Bi, X.-J., Cao, Z., Li, Y. and Yuan, Q., "Testing Lorentz invariance with the ultrahigh energy cosmic ray spectrum", Phys. Rev. D, 79, 083015 (2009). [DOI], [ADS], [arXiv:0812.0121]. (Cited on page 15.) 
[48] Biller, S. D. et al., "Limits to Quantum Gravity Effects on Energy Dependence of the Speed of Light from Observations of TeV Flares in Active Galaxies", Phys. Rev. Lett., 83, 2108-2111 (1999). [DOI], [arXiv:gr-qc/9810044]. (Cited on page 15.)

[49] Bize, S. et al., "Testing the Stability of Fundamental Constants with ${ }^{199} \mathrm{Hg}^{+}$Single-Ion Optical Clock", Phys. Rev. Lett., 90, 150802 (2003). [DOI], [physics/0212109]. (Cited on page 18.)

[50] Blanchet, L., "Second-post-Newtonian generation of gravitational radiation", Phys. Rev. D, 51, 2559-2583 (1995). [DOI], [gr-qc/9501030]. (Cited on page 60.)

[51] Blanchet, L., "Gravitational Radiation from Post-Newtonian Sources and Inspiralling Compact Binaries", Living Rev. Relativity, 17, lrr-2014-2 (2014). [DOI], [ADS], [arXiv:1310.1528 [gr-qc]]. URL (accessed 28 March 2014):

http://www.livingreviews.org/lrr-2014-2. (Cited on pages 60,61, 62, and 84.)

[52] Blanchet, L. and Damour, T., "Radiative gravitational fields in general relativity I. General structure of the field outside the source", Philos. Trans. R. Soc. London, Ser. A, 320, 379-430 (1986). [DOI]. (Cited on page 60.)

[53] Blanchet, L. and Damour, T., "Tail-transported temporal correlations in the dynamics of a gravitating system", Phys. Rev. D, 37, 1410-1435 (1988). [DOI]. (Cited on page 60.)

[54] Blanchet, L. and Damour, T., "Post-Newtonian generation of gravitational waves", Ann. Inst. Henri Poincare A, 50, 377-408 (1989). (Cited on page 60.)

[55] Blanchet, L. and Damour, T., "Hereditary effects in gravitational radiation", Phys. Rev. D, 46, 4304-4319 (1992). [DOI]. (Cited on page 60.)

[56] Blanchet, L., Damour, T., Iyer, B. R., Will, C. M. and Wiseman, A. G., "Gravitational-Radiation Damping of Compact Binary Systems to Second Post-Newtonian Order", Phys. Rev. Lett., 74, 35153518 (1995). [DOI], [gr-qc/9501027]. (Cited on page 61.)

[57] Blanchet, L. and Novak, J., "External field effect of modified Newtonian dynamics in the Solar system", Mon. Not. R. Astron. Soc., 412, 2530-2542 (2011). [DOI], [ADS], [arXiv:1010.1349 [astroph.CO]]. (Cited on page 40.)

[58] Blanchet, L. and Novak, J., "Testing MOND in the Solar System", arXiv, e-print, (2011). [ADS], [arXiv:1105.5815 [astro-ph.CO]]. (Cited on page 40.)

[59] Blanchet, L. and Sathyaprakash, B. S., "Signal analysis of gravitational wave tails", Class. Quantum Grav., 11, 2807-2831 (1994). [DOI]. (Cited on page 84.)

[60] Blanchet, L. and Sathyaprakash, B. S., "Detecting the tail effect in gravitational wave experiments", Phys. Rev. Lett., 74, 1067-1070 (1995). [DOI]. (Cited on page 84.)

[61] Blas, D., Pujolàs, O. and Sibiryakov, S., "Consistent extension of Hořava gravity", Phys. Rev. Lett., 104, 181302 (2010). [DOI], [ADS], [arXiv:0909.3525 [hep-th]]. (Cited on page 39.)

[62] Blas, D., Pujolàs, O. and Sibiryakov, S., "Models of non-relativistic quantum gravity: the good, the bad and the healthy", J. High Energy Phys., 4, 18 (2011). [DOI], [ADS], [arXiv:1007.3503 [hep-th]]. (Cited on page 39.)

[63] Blatt, S. et al., "New Limits on Coupling of Fundamental Constants to Gravity Using Sr87 Optical Lattice Clocks", Phys. Rev. Lett., 100, 140801 (2008). [DOI], [ADS], [arXiv:0801.1874 [physics.atomph]]. (Cited on pages 17, 18, and 19.)

[64] Bolton, A. S., Rappaport, S. and Burles, S., "Constraint on the post-Newtonian parameter $\gamma$ on galactic size scales", Phys. Rev. D, 74, 061501 (2006). [DOI], [ADS], [arXiv:astro-ph/0607657]. (Cited on page 44.) 
[65] Braginsky, V. B. and Panov, V. I., "Verification of the equivalence of inertial and gravitational mass", Sov. Phys. JETP, 34, 463-466 (1972). [DOI]. (Cited on page 10.)

[66] Braxmaier, C. et al., "Astrodynamical Space Test of Relativity using Optical Devices I (ASTROD I) - a class-M fundamental physics mission proposal for cosmic vision 2015-2025: 2010 Update", Exp. Astron., 34, 181-201 (2012). [DOI], [ADS], [arXiv:1104.0060 [gr-qc]]. (Cited on page 54.)

[67] Brecher, K., "Is the speed of light independent of the velocity of the source?", Phys. Rev. Lett., 39 1051-1054 (1977). [DOI]. (Cited on page 12.)

[68] Breton, R. P. et al., "Relativistic Spin Precession in the Double Pulsar", Science, 321, 104 (2008). [DOI], [ADS], [arXiv:0807.2644]. (Cited on page 72.)

[69] Brillet, A. and Hall, J. L., "Improved laser test of the isotropy of space", Phys. Rev. Lett., 42, 549-552 (1979). [DOI]. (Cited on pages 12 and 13.)

[70] Brunetti, M., Coccia, E., Fafone, V. and Fucito, F., "Gravitational-wave radiation from compact binary systems in the Jordan-Brans-Dicke theory", Phys. Rev. D, 59, 044027 (1999). [DOI], [grqc/9805056]. (Cited on page 82.)

[71] Burgay, M., "The double pulsar system in its 8th anniversary", in Science with Parkes at 50 Years Young, 31 Oct. -4 Nov., 2011, (ATNF / CSIRO, Australia, 2012). [ADS], [arXiv:1210.0985 [astroph.IM]]. URL (accessed 5 June 2014):

http://www.atnf.csiro.au/research/conferences/Parkes50th/. (Cited on page 72.)

[72] Burgay, M. et al., "An increased estimate of the merger rate of double neutron stars from observations of a highly relativistic system", Nature, 426, 531-533 (2003). [DOI], [ADS], [arXiv:astro-ph/0312071 [astro-ph]]. (Cited on page 72.)

[73] Carlip, S., "Model-dependence of Shapiro time delay and the 'speed of gravity/speed of light' controversy", Class. Quantum Grav., 21, 3803-3812 (2004). [DOI], [gr-qc/0403060]. (Cited on page 45.)

[74] Chamberlin, S. J. and Siemens, X., "Stochastic backgrounds in alternative theories of gravity: Overlap reduction functions for pulsar timing arrays", Phys. Rev. D, 85, 082001 (2012). [DOI], [ADS], [arXiv:1111.5661 [astro-ph.HE]]. (Cited on page 83.)

[75] Champeney, D. C., Isaak, G. R. and Khan, A. M., "An 'aether drift' experiment based on the Mössbauer effect", Phys. Lett., 7, 241-243 (1963). [DOI]. (Cited on page 12.)

[76] Chand, H., Petitjean, P., Srianand, R. and Aracil, B., "Probing the time-variation of the finestructure constant: Results based on Si IV doublets from a UVES sample", Astron. Astrophys., 430, 47-58 (2005). [DOI], [astro-ph/0408200]. (Cited on pages 18 and 19.)

[77] Chandrasekhar, S., "The Post-Newtonian Equations of Hydrodynamics in General Relativity.", Astrophys. J., 142, 1488-1540 (1965). [DOI], [ADS]. (Cited on page 58.)

[78] Chatziioannou, K., Yunes, N. and Cornish, N., "Model-independent test of general relativity: An extended post-Einsteinian framework with complete polarization content", Phys. Rev. D, 86, 022004 (2012). [DOI], [ADS], [arXiv:1204.2585 [gr-qc]]. (Cited on page 83.)

[79] Chiaverini, J., Smullin, S. J., Geraci, A. A., Weld, D. M. and Kapitulnik, A., "New experimental constraints on non-Newtonian forces below $100 \mu \mathrm{m}$ ", Phys. Rev. Lett., 90, 151101 (2003). [DOI], [hep-ph/0209325]. (Cited on page 27.)

[80] Chou, C. W., Hume, D. B., Rosenband, T. and Wineland, D. J., "Optical Clocks and Relativity", Science, 329, 1630-1633 (2010). [DOI], [ADS]. (Cited on page 17.)

[81] Chupp, T. E., Hoare, R. J., Loveman, R. A., Oteiza, E. R., Richardson, J. M., Wagshul, M. E. and Thompson, A. K., "Results of a new test of local Lorentz invariance: A search for mass anisotropy in ${ }^{21} \mathrm{Ne} "$, Phys. Rev. Lett., 63, 1541-1545 (1989). [DOI]. (Cited on page 13.) 
[82] Ciufolini, I., "The 1995-99 measurements of the Lense-Thirring effect using laser-ranged satellites", Class. Quantum Grav., 17, 2369-2380 (2000). [DOI]. (Cited on page 52.)

[83] Ciufolini, I., Chieppa, F., Lucchesi, D. and Vespe, F., "Test of Lense-Thirring orbital shift due to spin", Class. Quantum Grav., 14, 2701-2726 (1997). [DOI]. (Cited on page 52.)

[84] Ciufolini, I., Moreno Monge, B., Paolozzi, A., Koenig, R., Sindoni, G., Michalak, G. and Pavlis, E. C., "Monte Carlo simulations of the LARES space experiment to test General Relativity and fundamental physics", Class. Quantum Grav., 30, 235009 (2013). [DOI], [ADS], [arXiv:1310.2601 [gr-qc]]. (Cited on page 52.)

[85] Ciufolini, I., Paolozzi, A., Pavlis, E. C., Ries, J., Koenig, R., Matzner, R., Sindoni, G. and Neumeyer, H., "Testing gravitational physics with satellite laser ranging", Eur. Phys. J. Plus, 126, 72 (2011). [DOI], [ADS]. (Cited on page 52.)

[86] Ciufolini, I. and Pavlis, E. C., "A confirmation of the general relativistic prediction of the LenseThirring effect", Nature, 431, 958-960 (2004). [DOI], [ADS]. (Cited on page 52.)

[87] Ciufolini, I., Pavlis, E. C., Chieppa, F., Fernandes-Vieira, E. and Pérez-Mercader, J., "Test of general relativity and measurement of the Lense-Thirring effect with two Earth satellites", Science, 279, 2100-2103 (1998). [DOI]. (Cited on page 52.)

[88] Ciufolini, I., Pavlis, E. C. and Peron, R., "Determination of frame-dragging using Earth gravity models from CHAMP and GRACE", New Astron., 11, 527-550 (2006). [DOI], [ADS]. (Cited on page 52.)

[89] Clifton, T., Barrow, J. D. and Scherrer, R. J., "Constraints on the variation of $G$ from primordial nucleosynthesis", Phys. Rev. D, 71, 123526 (2005). [DOI], [astro-ph/0504418]. (Cited on page 87.)

[90] Coc, A., Olive, K. A., Uzan, J.-P. and Vangioni, E., "Big bang nucleosynthesis constraints on scalartensor theories of gravity", Phys. Rev. D, 73, 083525 (2006). [DOI], [astro-ph/0601299]. (Cited on page 87.)

[91] Coley, A., "Schiff's Conjecture on Gravitation", Phys. Rev. Lett., 49, 853-855 (1982). [DOI]. (Cited on page 20.)

[92] Colladay, D. and Kostelecký, V. A., "CPT violation and the standard model", Phys. Rev. D, 55, 6760-6774 (1997). [DOI], [arXiv:hep-ph/9703464]. (Cited on pages 13 and 24.)

[93] Colladay, D. and Kostelecký, V. A., "Lorentz-violating extension of the standard model", Phys. Rev. $D, \mathbf{5 8}, 116002$ (1998). [DOI], [arXiv:hep-ph/9809521]. (Cited on pages 13 and 24.)

[94] Copi, C. J., Davis, A. N. and Krauss, L. M., "New Nucleosynthesis Constraint on the Variation of G", Phys. Rev. Lett., 92, 171301 (2004). [DOI], [arXiv:astro-ph/0311334]. (Cited on page 50.)

[95] Crelinsten, J., Einstein's Jury: The Race to Test Relativity, (Princeton University Press, Princeton, 2006). (Cited on pages 16 and 43.)

[96] Creminelli, P., Nicolis, A., Papucci, M. and Trincherini, E., "Ghosts in massive gravity", J. High Energy Phys., 2005(09), 003 (2005). [DOI], [hep-th/0505147]. (Cited on page 41.)

[97] Cutler, C. and Flanagan, É.É., "Gravitational waves from merging compact binaries: How accurately can one extract the binary's parameters from the inspiral wave form?", Phys. Rev. D, 49, 2658-2697 (1994). [DOI], [arXiv:gr-qc/9402014]. (Cited on page 83.)

[98] Cutler, C., Hiscock, W. A. and Larson, S. L., "LISA, binary stars, and the mass of the graviton", Phys. Rev. D, 67, 024015 (2003). [DOI], [ADS], [arXiv:gr-qc/0209101]. (Cited on page 86.) 
[99] Cutler, C. et al., "The Last Three Minutes: Issues in Gravitational-Wave Measurements of Coalescing Compact Binaries", Phys. Rev. Lett., 70, 2984-2987 (1993). [DOI], [astro-ph/9208005]. (Cited on page 83.)

[100] Damour, T., "The problem of motion in Newtonian and Einsteinian gravity", in Hawking, S. W. and Israel, W., eds., Three Hundred Years of Gravitation, pp. 128-198, (Cambridge University Press, Cambridge; New York, 1987). [ADS]. (Cited on pages 56, 57, and 60.)

[101] Damour, T. and Dyson, F. J., "The Oklo bound on the time variation of the fine-structure constant revisited", Nucl. Phys. B, 480, 37-54 (1996). [DOI], [hep-ph/9606486]. (Cited on pages 18 and 19.)

[102] Damour, T. and Esposito-Farèse, G., "Tensor-multi-scalar theories of gravitation", Class. Quantum Grav., 9, 2093-2176 (1992). [DOI], [ADS]. (Cited on pages 35, 36, and 66.)

[103] Damour, T. and Esposito-Farèse, G., "Nonperturbative strong-field effects in tensor-scalar theories of gravitation", Phys. Rev. Lett., 70, 2220-2223 (1993). [DOI], [ADS]. (Cited on page 37.)

[104] Damour, T. and Esposito-Farèse, G., "Tensor-scalar gravity and binary-pulsar experiments", Phys. Rev. D, 54, 1474-1491 (1996). [DOI], [ADS], [gr-qc/9602056]. (Cited on page 37.)

[105] Damour, T. and Esposito-Farèse, G., "Testing gravity to second post-Newtonian order: A fieldtheory approach", Phys. Rev. D, 53, 5541-5578 (1996). [DOI], [ADS], [gr-qc/9506063]. (Cited on page 62.)

[106] Damour, T. and Esposito-Farèse, G., "Gravitational-wave versus binary-pulsar tests of strong-field gravity", Phys. Rev. D, 58, 042001 (1998). [DOI], [ADS], [arXiv:gr-qc/9803031]. (Cited on pages 76 and 84.)

[107] Damour, T., Gopakumar, A. and Iyer, B. R., "Phasing of gravitational waves from inspiralling eccentric binaries", Phys. Rev. D, 70, 064028 (2004). [DOI], [gr-qc/0404128]. (Cited on page 84.)

[108] Damour, T. and Iyer, B. R., "Post-Newtonian generation of gravitational waves. II. The spin moments", Ann. Inst. Henri Poincare A, 54, 115-164 (1991). (Cited on page 60.)

[109] Damour, T., Jaranowski, P. and Schäfer, G., "Poincaré invariance in the ADM Hamiltonian approach to the general relativistic two-body problem", Phys. Rev. D, 62, 021501(R) (2000). [gr-qc/0003051]. Erratum: Phys. Rev. D, 63, 029903(E) (2001). (Cited on page 60.)

[110] Damour, T., Jaranowski, P. and Schäfer, G., "Dimensional regularization of the gravitational interaction of point masses", Phys. Lett. B, 513, 147-155 (2001). [DOI], [gr-qc/0105038]. (Cited on page 60.)

[111] Damour, T., Jaranowski, P. and Schäfer, G., "Equivalence between the ADM-Hamiltonian and the harmonic-coordinates approaches to the third post-Newtonian dynamics of compact binaries", Phys. Rev. D, 63, 044021 (2001). [DOI], [gr-qc/0010040]. Erratum: Phys. Rev. D, 66, 029901(E) (2002). (Cited on page 60.)

[112] Damour, T. and Nordtvedt Jr, K. L., "General relativity as a cosmological attractor of tensor-scalar theories", Phys. Rev. Lett., 70, 2217-2219 (1993). [DOI]. (Cited on page 36.)

[113] Damour, T. and Nordtvedt Jr, K. L., "Tensor-scalar cosmological models and their relaxation toward general relativity", Phys. Rev. D, 48, 3436-3450 (1993). [DOI]. (Cited on page 36.)

[114] Damour, T., Piazza, F. and Veneziano, G., "Runaway dilaton and equivalence principle violations", Phys. Rev. Lett., 89, 081601 (2002). [DOI], [arXiv:gr-qc/0204094]. (Cited on page 37.)

[115] Damour, T., Piazza, F. and Veneziano, G., "Violations of the equivalence principle in a dilatonrunaway scenario", Phys. Rev. D, 66, 046007 (2002). [DOI], [arXiv:hep-th/0205111]. (Cited on page 37.) 
[116] Damour, T. and Pichon, B., "Big bang nucleosynthesis and tensor-scalar gravity", Phys. Rev. D, 59, 123502 (1999). [DOI], [astro-ph/9807176]. (Cited on page 87.)

[117] Damour, T. and Polyakov, A. M., "The string dilaton and a least coupling principle", Nucl. Phys. B, 423, 532-558 (1994). [DOI], [arXiv:hep-th/9401069]. (Cited on pages 26 and 37.)

[118] Damour, T. and Schäfer, G., "New tests of the strong equivalence principle using binary-pulsar data", Phys. Rev. Lett., 66, 2549-2552 (1991). [DOI]. (Cited on page 49.)

[119] Damour, T. and Taylor, J. H., "Strong-field tests of relativistic gravity and binary pulsars", Phys. Rev. D, 45, 1840-1868 (1992). [DOI]. (Cited on page 69.)

[120] Damour, T. and Vokrouhlický, D., "Equivalence principle and the Moon", Phys. Rev. D, 53, 41774201 (1996). [DOI], [gr-qc/9507016]. (Cited on page 48.)

[121] Daniel, S. F., Linder, E. V., Smith, T. L., Caldwell, R. R., Cooray, A., Leauthaud, A. and Lombriser, L., "Testing general relativity with current cosmological data", Phys. Rev. D, 81, 123508 (2010). [DOI], [ADS], [arXiv:1002.1962 [astro-ph.CO]]. (Cited on page 88.)

[122] De Felice, A. and Tsujikawa, S., " $f(R)$ Theories", Living Rev. Relativity, 13, lrr-2010-3 (2010). [DOI], [ADS], [arXiv:1002.4928]. URL (accessed 28 March 2014):

http://www.livingreviews.org/lrr-2010-3. (Cited on page 37.)

[123] de Rham, C., "Massive Gravity", Living Rev. Relativity, forthcoming (2014). [ADS], [arXiv:1401.4173 [hep-th]]. (Cited on page 41.)

[124] de Sitter, W., "On Einstein's theory of gravitation and its astronomical consequences. Second paper", Mon. Not. R. Astron. Soc., 77, 155-184 (1916). [ADS]. (Cited on page 57.)

[125] Deffayet, C., Dvali, G., Gabadadze, G. and Vainshtein, A. I., "Nonperturbative continuity in graviton mass versus perturbative discontinuity", Phys. Rev. D, 65, 044026 (2002). [DOI], [arXiv:hepth/0106001]. (Cited on page 41.)

[126] Deller, A. T., Verbiest, J. P. W., Tingay, S. J. and Bailes, M., "Extremely High Precision VLBI Astrometry of PSR J0437-4715 and Implications for Theories of Gravity", Astrophys. J. Lett., 685, L67-L70 (2008). [DOI], [ADS], [arXiv:0808.1594]. (Cited on page 50.)

[127] Demorest, P. B., Pennucci, T., Ransom, S. M., Roberts, M. S. E. and Hessels, J. W. T., "A twosolar-mass neutron star measured using Shapiro delay", Nature, 467, 1081-1083 (2010). [DOI], [ADS], [arXiv:1010.5788 [astro-ph.HE]]. (Cited on page 73.)

[128] Di Casola, E., Liberati, S. and Sonego, S., "Nonequivalence of equivalence principles", arXiv, e-print, (2013). [ADS], [arXiv:1310.7426 [gr-qc]]. (Cited on pages 10 and 30.)

[129] Dick, R., "Inequivalence of Jordan and Einstein frame: What is the low energy gravity in string theory?", Gen. Relativ. Gravit., 30, 435-444 (1998). [DOI]. (Cited on page 25.)

[130] Dicke, R. H., "Experimental relativity", in DeWitt, C. M. and DeWitt, B. S., eds., Relativity, Groups and Topology. Relativité, Groupes et Topologie, Lectures delivered at Les Houches during the 1963 session of the Summer School of Theoretical Physics, University of Grenoble, pp. 165-313, (Gordon and Breach, New York; London, 1964). (Cited on page 9.)

[131] Dicke, R. H., Gravitation and the Universe, Memoirs of the American Philosophical Society. Jayne Lecture for 1969, 78, (American Philosophical Society, Philadelphia, 1970). (Cited on page 10.)

[132] Dickey, J. O. et al., "Lunar Laser Ranging: A Continuing Legacy of the Apollo Program", Science, 265, 482-490 (1994). [DOI]. (Cited on pages 48, 50, and 53.) 
[133] Doeleman, S. et al., "Imaging an Event Horizon: submm-VLBI of a Super Massive Black Hole", in Astro2010: The Astronomy and Astrophysics Decadal Survey - Science White Papers, p. 68, (National Academy of Sciences, Washington, DC, 2009). [ADS], [arXiv:0906.3899 [astro-ph.CO]]. URL (accessed 10 June 2014):

http://sites.nationalacademies.org/bpa/BPA_050603. (Cited on page 87.)

[134] Dossett, J. N. and Ishak, M., "Spatial curvature and cosmological tests of general relativity", Phys. Rev. D, 86, 103008 (2012). [DOI], [ADS], [arXiv:1205.2422 [astro-ph.CO]]. (Cited on page 88.)

[135] Dossett, J. N., Ishak, M. and Moldenhauer, J., "Testing general relativity at cosmological scales: Implementation and parameter correlations", Phys. Rev. D, 84, 123001 (2011). [DOI], [ADS], [arXiv:1109.4583 [astro-ph.CO]]. (Cited on page 88.)

[136] Drever, R. W. P., "A search for anisotropy of inertial mass using a free precession technique", Philos. Mag., 6, 683-687 (1961). [DOI]. (Cited on page 13.)

[137] Dyda, S., Flanagan, É. É. and Kamionkowski, M., "Vacuum instability in Chern-Simons gravity", Phys. Rev. D, 86, 124031 (2012). [DOI], [ADS], [arXiv:1208.4871 [gr-qc]]. (Cited on page 41.)

[138] Dyson, F. J., "The Fundamental Constants and Their Time Variation", in Salam, A. and Wigner, E. P., eds., Aspects of Quantum Theory, pp. 213-236, (Cambridge University Press, Cambridge; New York, 1972). [Google Books]. (Cited on page 18.)

[139] Eardley, D. M., "Observable effects of a scalar gravitational field in a binary pulsar", Astrophys. J. Lett., 196, L59-L62 (1975). [DOI], [ADS]. (Cited on pages 62, 63, and 74.)

[140] Eddington, A. and Clark, G. L., "The Problem of n Bodies in General Relativity Theory", Proc. R. Soc. London, Ser. A, 166, 465-475 (1938). [DOI], [ADS]. (Cited on page 57.)

[141] Eddington, A. S., "The Propagation of Gravitational Waves", Proc. R. Soc. London, Ser. A, 102, 268-282 (1922). [DOI], [ADS]. (Cited on page 57.)

[142] Ehlers, J., Rosenblum, A., Goldberg, J. N. and Havas, P., "Comments on gravitational radiation damping and energy loss in binary systems", Astrophys. J. Lett., 208, L77-L81 (1976). [DOI], [ADS]. (Cited on page 58.)

[143] Einstein, A., "Näherungsweise Integration der Feldgleichungen der Gravitation", Sitzungsber. K. Preuss. Akad. Wiss., Phys.-Math. Kl., 1916, 688-696 (1916). [ADS]. Online version (accessed 5 June 2014): http://echo.mpiwg-berlin.mpg.de/MPIWG:RA6W5W65. (Cited on page 57.)

[144] Einstein, A., "Über Gravitationswellen", Sitzungsber. K. Preuss. Akad. Wiss., 1918, 154-167 (1918). [ADS]. (Cited on page 57.)

[145] Einstein, A., Infeld, L. and Hoffmann, B., "The Gravitational Equations and the Problem of Motion", Ann. Math., 39, 65-100 (1938). [DOI], [ADS]. (Cited on page 57.)

[146] Einstein, A. and Rosen, N., "On Gravitational Waves", J. Franklin Inst., 223, 43-54 (1937). [DOI], [ADS]. (Cited on page 57.)

[147] Eling, C. and Jacobson, T., "Static post-Newtonian equivalence of general relativity and gravity with a dynamical preferred frame", Phys. Rev. D, 69, 064005 (2004). [DOI], [gr-qc/0310044]. (Cited on page 38.)

[148] Eötvös, R. v., Pekár, V. and Fekete, E., "Beitrage zum Gesetze der Proportionalität von Trägheit und Gravität", Ann. Phys. (Leipzig), 68, 11-66 (1922). [DOI], [ADS]. (Cited on page 10.)

[149] Everitt, C. W. F. et al., "Gravity Probe B: Final Results of a Space Experiment to Test General Relativity", Phys. Rev. Lett., 106, 221101 (2011). [DOI], [ADS], [arXiv:1105.3456 [gr-qc]]. (Cited on page 52.) 
[150] Famaey, B. and McGaugh, S. S., "Modified Newtonian Dynamics (MOND): Observational Phenomenology and Relativistic Extensions", Living Rev. Relativity, 15, lrr-2012-10 (2012). [DOI], [ADS], [arXiv:1112.3960 [astro-ph.CO]]. URL (accessed 28 March 2014): http://www.livingreviews.org/lrr-2012-10. (Cited on page 40.)

[151] Farley, F. J. M., Bailey, J., Brown, R. C. A., Giesch, M., Jöstlein, H., van der Meer, S., Picasso, E. and Tannenbaum, M., "The Anomalous Magnetic Moment of the Negative Muon", Nuovo Cimento, 45, 281-286 (1966). [DOI]. (Cited on page 12.)

[152] Fienga, A., Laskar, J., Kuchynka, P., Manche, H., Desvignes, G., Gastineau, M., Cognard, I. and Theureau, G., "The INPOP10a planetary ephemeris and its applications in fundamental physics", Celest. Mech. Dyn. Astron., 111, 363-385 (2011). [DOI], [ADS], [arXiv:1108.5546 [astro-ph.EP]]. (Cited on page 47.)

[153] Finn, L. S. and Chernoff, D. F., "Observing binary inspiral in gravitational radiation: One interferometer", Phys. Rev. D, 47, 2198-2219 (1993). [DOI], [arXiv:gr-qc/9301003]. (Cited on page 83.)

[154] Finn, L. S. and Sutton, P. J., "Bounding the mass of the graviton using binary pulsar observations", Phys. Rev. D, 65, 044022 (2002). [DOI], [ADS], [arXiv:gr-qc/0109049]. (Cited on page 86.)

[155] Fischbach, E., Gillies, G. T., Krause, D. E., Schwan, J. G. and Talmadge, C. L., "Non-Newtonian gravity and new weak forces: An index of measurements and theory", Metrologia, 29, 213-260 (1992). [DOI]. (Cited on pages 10 and 27.)

[156] Fischbach, E., Sudarsky, D., Szafer, A., Talmadge, C. L. and Aronson, S. H., "Reanalysis of the Eötvös experiment", Phys. Rev. Lett., 56, 3-6 (1986). [DOI]. Erratum: Phys. Rev. Lett. 56 (1986) 1427. (Cited on page 26.)

[157] Fischbach, E. and Talmadge, C. L., "Six years of the fifth force", Nature, 356, 207-215 (1992). [DOI]. (Cited on page 27.)

[158] Fischbach, E. and Talmadge, C. L., The Search for Non-Newtonian Gravity, (Springer, New York, 1998). [Google Books]. (Cited on page 27.)

[159] Fischer, M. et al., "New limits on the drift of fundamental constants from laboratory measurements", Phys. Rev. Lett., 92, 230802 (2004). [DOI], [physics/0312086]. (Cited on page 18.)

[160] Fock, V. A., The Theory of Space, Time and Gravitation, (Pergamon Press, Oxford; New York, 1964), 2nd rev. edition. (Cited on page 58.)

[161] Fomalont, E., Kopeikin, S., Lanyi, G. and Benson, J., "Progress in Measurements of the Gravitational Bending of Radio Waves Using the VLBA", Astrophys. J., 699, 1395-1402 (2009). [DOI], [ADS], [arXiv:0904.3992 [astro-ph.CO]]. (Cited on page 44.)

[162] Fomalont, E. B. and Kopeikin, S. M., "The measurement of the light deflection from Jupiter: experimental results", Astrophys. J., 598, 704-711 (2003). [DOI], [astro-ph/0302294]. (Cited on page 45.)

[163] Foster, B. Z. and Jacobson, T., "Post-Newtonian parameters and constraints on Einstein-aether theory", Phys. Rev. D, 73, 064015 (2006). [DOI], [arXiv:gr-qc/0509083 [gr-qc]]. (Cited on page 38.)

[164] Freire, P. C. C. et al., "The relativistic pulsar-white dwarf binary PSR J1738+0333 - II. The most stringent test of scalar-tensor gravity", Mon. Not. R. Astron. Soc., 423, 3328-3343 (2012). [DOI], [ADS], [arXiv:1205.1450 [astro-ph.GA]]. (Cited on pages 72, 75, 77, and 78.)

[165] Froeschlé, M., Mignard, F. and Arenou, F., "Determination of the PPN parameter $\gamma$ with the Hipparcos data", in Proceedings from the Hipparcos Venice '97 Symposium, Held on 13-16 May 1997, (ESA, Noordwijk, Netherlands, 1997). URL (accessed 10 June 2014):

http://www.rssd.esa.int/?project=HIPPARCOS\&page=venice97. (Cited on page 44.) 
[166] Fujii, Y., "Oklo Constraint on the Time-Variability of the Fine-Structure Constant", in Karshenboim, S. G. and Peik, E., eds., Astrophysics, Clocks and Fundamental Constants, 302nd WE-HeraeusSeminar, June 2003, Bad Honnef, Germany, Lecture Notes in Physics, 648, pp. 167-185, (Springer, Berlin; New York, 2004). [hep-ph/0311026]. (Cited on pages 18 and 19.)

[167] Fujii, Y. and Maeda, K.-I., The Scalar-Tensor Theory of Gravitation, Cambridge Monographs on Mathematical Physics, (Cambridge University Press, Cambridge; New York, 2007). [ADS]. (Cited on page 37.)

[168] Fujita, R., "Gravitational Waves from a Particle in Circular Orbits around a Schwarzschild Black Hole to the 22nd Post-Newtonian Order", Prog. Theor. Phys., 128, 971-992 (2012). [DOI], [ADS], [arXiv:1211.5535 [gr-qc]]. (Cited on page 62.)

[169] "Gaia: Science Home Page", project homepage, ESA. URL (accessed 28 March 2014): http://www.cosmos.esa.int/web/gaia/. (Cited on page 54.)

[170] Gair, J. R., Vallisneri, M., Larson, S. L. and Baker, J. G., "Testing General Relativity with LowFrequency, Space-Based Gravitational-Wave Detectors", Living Rev. Relativity, 16, lrr-2013-7 (2013). [DOI], [ADS], [arXiv:1212.5575 [gr-qc]]. URL (accessed 28 March 2014): http://www.livingreviews.org/lrr-2013-7. (Cited on page 79.)

[171] Gasperini, M., "On the response of gravitational antennas to dilatonic waves", Phys. Lett. B, 470, 67-72 (1999). [DOI], [gr-qc/9910019]. (Cited on page 82.)

[172] Geraci, A. A., Smullin, S. J., Weld, D. M., Chiaverini, J. and Kapitulnik, A., "Improved constraints on non-Newtonian forces at 10 microns", Phys. Rev. D, 78, 022002 (2008). [DOI], [ADS], [arXiv:0802.2350 [hep-ex]]. (Cited on page 27.)

[173] Gleiser, R. J. and Kozameh, C. N., "Astrophysical limits on quantum gravity motivated birefringence", Phys. Rev. D, 64, 083007 (2001). [DOI], [arXiv:gr-qc/0102093]. (Cited on page 15.)

[174] Godone, A., Novero, C. and Tavella, P., "Null gravitational redshift experiment with nonidentical atomic clocks", Phys. Rev. D, 51, 319-323 (1995). [DOI]. (Cited on page 17.)

[175] Gopakumar, A. and Iyer, B. R., "Gravitational waves from inspiraling compact binaries: Angular momentum flux, evolution of the orbital elements and the waveform to the second post-Newtonian order", Phys. Rev. D, 56, 7708-7731 (1997). [DOI], [arXiv:gr-qc/9710075]. (Cited on page 84.)

[176] Gourgoulhon, E., 3+1 Formalism in General Relativity: Bases of Numerical Relativity, Lecture Notes in Physics, 846, (Springer, Berlin; New York, 2012). [DOI], [ADS], [arXiv:gr-qc/0703035 [grqc]]. (Cited on page 55.)

[177] "Gravity Probe B: Testing Einstein's Universe", project homepage, Stanford University. URL (accessed 28 March 2014):

http://einstein.stanford.edu/. (Cited on page 51.)

[178] Grishchuk, L. P. and Kopeikin, S. M., "The Motion of a Pair of Gravitating Bodies Including the Radiation Reaction Force", Sov. Astron. Lett., 9, 230-232 (1983). [ADS]. (Cited on page 60.)

[179] Guéna, J., Abgrall, M., Rovera, D., Rosenbusch, P., Tobar, M. E., Laurent, P., Clairon, A. and Bize, S., "Improved Tests of Local Position Invariance Using Rb87 and Cs133 Fountains", Phys. Rev. Lett., 109, 080801 (2012). [DOI], [ADS], [arXiv:1205.4235 [physics.atom-ph]]. (Cited on pages 17, 18, and 19.)

[180] Guenther, D. B., Krauss, L. M. and Demarque, P., "Testing the Constancy of the Gravitational Constant Using Helioseismology", Astrophys. J., 498, 871-876 (1998). [DOI], [ADS]. (Cited on page 50.) 
[181] Haugan, M. P., "Energy conservation and the principle of equivalence", Ann. Phys. (N.Y.), 118, 156-186 (1979). [DOI]. (Cited on page 20.)

[182] Haugan, M. P. and Lämmerzahl, C., "On the interpretation of Michelson-Morley experiments", Phys. Lett. A, 282, 223-229 (2001). [gr-qc/0103052]. (Cited on page 13.)

[183] Haugan, M. P. and Lämmerzahl, C., "Principles of Equivalence: Their Role in Gravitation Physics and Experiments That Test Them", in Lämmerzahl, C., Everitt, C. W. F. and Hehl, F. W., eds., Gyros, Clocks, Interferometers...: Testing Relativistic Gravity in Space, Proceedings of a meeting held at Bad Honnef, Germany, 21-7 August 1999, Lecture Notes in Physics, 562, pp. 195-212, (Springer, Berlin; New York, 2001). [DOI], [ADS], [gr-qc/0103067]. (Cited on page 10.)

[184] Haugan, M. P. and Will, C. M., "Modern tests of special relativity", Phys. Today, 40, 69-76 (1987). [DOI]. (Cited on page 13.)

[185] Hawking, S. W., "Black holes in the Brans-Dicke Theory of gravitation", Commun. Math. Phys., 25, 167-171 (1972). [DOI], [ADS]. (Cited on pages 63 and 66.)

[186] Hees, A., Folkner, W. M., Jacobson, R. A. and Park, R. S., "Constraints on MOND theory from radio tracking data of the Cassini spacecraft", arXiv, e-print, (2014). [ADS], [arXiv:1402.6950 [gr-qc]]. (Cited on page 40.)

[187] Hellings, R. W. and Nordtvedt Jr, K. L., "Vector-Metric Theory of Gravity", Phys. Rev. D, 7, 3593-3602 (1973). [DOI]. (Cited on page 38.)

[188] Hinterbichler, K., "Theoretical aspects of massive gravity", Rev. Mod. Phys., 84, 671-710 (2012). [DOI], [ADS], [arXiv:1105.3735 [hep-th]]. (Cited on page 41.)

[189] Hojjati, A., Zhao, G.-B., Pogosian, L., Silvestri, A., Crittenden, R. and Koyama, K., "Cosmological tests of general relativity: A principal component analysis", Phys. Rev. D, 85, 043508 (2012). [DOI], [ADS], [arXiv:1111.3960 [astro-ph.CO]]. (Cited on page 88.)

[190] Hořava, P., "Quantum gravity at a Lifshitz point", Phys. Rev. D, 79, 084008 (2009). [DOI], [ADS], [arXiv:0901.3775 [hep-th]]. (Cited on page 39.)

[191] Horbatsch, M. W. and Burgess, C. P., "Cosmic black-hole hair growth and quasar OJ287", J. Cosmol. Astropart. Phys., 2012(05), 010 (2012). [DOI], [ADS], [arXiv:1111.4009 [gr-qc]]. (Cited on page 67.)

[192] Hoyle, C. D., Kapner, D. J., Heckel, B. R., Adelberger, E. G., Gundlach, J. H., Schmidt, U. and Swanson, H. E., "Submillimeter tests of the gravitational inverse-square law", Phys. Rev. D, 70, 042004 (2004). [DOI], [arXiv:hep-ph/0405262]. (Cited on page 27.)

[193] Hoyle, C. D., Schmidt, U., Heckel, B. R., Adelberger, E. G., Gundlach, J. H., Kapner, D. J. and Swanson, H. E., "Submillimeter Test of the Gravitational Inverse-Square Law: A Search for 'large' Extra Dimensions", Phys. Rev. Lett., 86, 1418-1421 (2001). [DOI], [arXiv:hep-ph/0011014]. (Cited on page 27.)

[194] Hughes, V. W., Robinson, H. G. and Beltran-Lopez, V., "Upper limit for the anisotropy of inertial mass from nuclear resonance experiments", Phys. Rev. Lett., 4, 342-344 (1960). [DOI]. (Cited on page 13.)

[195] Hulse, R. A., "Nobel Lecture: The discovery of the binary pulsar", Rev. Mod. Phys., 66, 699-710 (1994). [DOI]. (Cited on page 68.)

[196] Hulse, R. A. and Taylor, J. H., "Discovery of a pulsar in a binary system", Astrophys. J. Lett., 195, L51-L53 (1975). [DOI], [ADS]. (Cited on page 58.)

[197] Iorio, L., "On the reliability of the so-far performed tests for measuring the Lense-Thirring effect with the LAGEOS satellites", New Astron., 10, 603-615 (2005). [DOI], [gr-qc/0411024]. (Cited on page 52.) 
[198] Itoh, Y. and Futamase, T., "New derivation of a third post-Newtonian equation of motion for relativistic compact binaries without ambiguity", Phys. Rev. D, 68, 121501(R) (2003). [DOI], [grqc/0310028]. (Cited on page 60.)

[199] Ivanchik, A., Petitjean, P., Varshalovich, D., Aracil, B., Srianand, R., Chand, H., Ledoux, C. and Boissé, P., "A new constraint on the time dependence of the proton-to-electron mass ratio: Analysis of the Q 0347-383 and Q 0405-443 spectra", Astron. Astrophys., 440, 45-52 (2005). [DOI], [astroph/0507174]. (Cited on page 19.)

[200] Ives, H. E. and Stilwell, G. R., "An experimental study of the rate of a moving atomic clock", J. Opt. Soc. Am., 28, 215-226 (1938). [DOI]. (Cited on page 12.)

[201] Jackiw, R. and Pi, S.-Y., "Chern-Simons modification of general relativity", Phys. Rev. D, 68, 104012 (2003). [DOI], [ADS], [gr-qc/0308071]. (Cited on page 41.)

[202] Jacobson, T., "Primordial Black Hole Evolution in Tensor-Scalar Cosmology", Phys. Rev. Lett., 83, 2699-2702 (1999). [DOI], [ADS], [astro-ph/9905303]. (Cited on page 67.)

[203] Jacobson, T., "Undoing the twist: The Hořava limit of Einstein-æther theory", Phys. Rev. D, 89, 081501 (2014). [DOI], [ADS], [arXiv:1310.5115 [gr-qc]]. (Cited on page 39.)

[204] Jacobson, T. and Mattingly, D., "Gravity with a dynamical preferred frame", Phys. Rev. D, 64, 024028 (2001). [DOI], [ADS], [arXiv:gr-qc/0007031]. (Cited on page 38.)

[205] Jacobson, T. and Mattingly, D., "Einstein-aether waves", Phys. Rev. D, 70, 024003 (2004). [DOI], [arXiv:gr-qc/0402005 [gr-qc]]. (Cited on pages 38 and 82.)

[206] Jaranowski, P. and Schäfer, G., "3rd post-Newtonian higher order Hamilton dynamics for two-body point-mass systems", Phys. Rev. D, 57, 7274-7291 (1998). [DOI], [gr-qc/9712075]. Erratum: Phys. Rev. D, 63 (2001) 029902. (Cited on page 60.)

[207] Jaranowski, P. and Schäfer, G., "Binary black-hole problem at the third post-Newtonian approximation in the orbital motion: Static part", Phys. Rev. D, 60, 124003 (1999). [DOI], [gr-qc/9906092]. (Cited on page 60.)

[208] Jaseja, T. S., Javan, A., Murray, J. and Townes, C. H., "Test of special relativity or of the isotropy of space by use of infrared masers", Phys. Rev., 133, A1221-A1225 (1964). [DOI]. (Cited on page 12.)

[209] Jones, D. I., "Bounding the Mass of the Graviton Using Eccentric Binaries", Astrophys. J. Lett., 618, L115-L118 (2005). [DOI], [ADS], [arXiv:gr-qc/0411123]. (Cited on page 86.)

[210] Kanekar, N., Langston, G. I., Stocke, J. T., Carilli, C. L. and Menten, K. M., "Constraining Fundamental Constant Evolution with H I and OH Lines", Astrophys. J. Lett., 746, L16 (2012). [DOI], [ADS], [arXiv:1201.3372 [astro-ph.CO]]. (Cited on pages 18 and 19.)

[211] Kapner, D. J., Cook, T. S., Adelberger, E. G., Gundlach, J. H., Heckel, B. R., Hoyle, C. D. and Swanson, H. E., "Tests of the Gravitational Inverse-Square Law below the Dark-Energy Length Scale", Phys. Rev. Lett., 98, 021101 (2007). [DOI], [ADS], [hep-ph/0611184]. (Cited on page 27.)

[212] Katz, J. I., "Comment on 'Indication, from Pioneer 10/11, Galileo, and Ulysses Data, of an Apparent Anomalous, Weak, Long-Range Acceleration"', Phys. Rev. Lett., 83, 1892 (1999). [DOI], [ADS], [grqc/9809070]. (Cited on page 27.)

[213] Kennefick, D., "Einstein versus the Physical Review", Phys. Today, 58, 43-48 (2005). [DOI], [ADS]. (Cited on page 57.)

[214] Kennefick, D., Traveling at the Speed of Thought: Einstein and the Quest for Gravitational Waves, (Princeton University Press, Princeton; Woodstock, UK, 2007). [ADS], [Google Books]. (Cited on page 58.) 
[215] Kennefick, D., "Testing relativity from the 1919 eclipse - a question of bias", Phys. Today, 62, 37 (2009). [DOI], [ADS]. (Cited on page 43.)

[216] Khoury, J. and Weltman, A., "Chameleon Fields: Awaiting Surprises for Tests of Gravity in Space", Phys. Rev. Lett., 93, 171104 (2004). [DOI], [ADS], [astro-ph/0309300]. (Cited on page 37.)

[217] King, J. A., Webb, J. K., Murphy, M. T., Flambaum, V. V., Carswell, R. F., Bainbridge, M. B., Wilczynska, M. R. and Koch, F. E., "Spatial variation in the fine-structure constant - new results from VLT/UVES", Mon. Not. R. Astron. Soc., 422, 3370-3414 (2012). [DOI], [ADS], [arXiv:1202.4758 [astro-ph.CO]]. (Cited on pages 18 and 19.)

[218] Klimchitskaya, G. L., Mohideen, U. and Mostepanenko, V. M., "Constraints on corrections to Newtonian gravity from two recent measurements of the Casimir interaction between metallic surfaces", Phys. Rev. D, 87, 125031 (2013). [DOI], [ADS], [arXiv:1306.4979 [gr-qc]]. (Cited on page 27.)

[219] Kokkotas, K. D. and Schmidt, B. G., "Quasi-Normal Modes of Stars and Black Holes", Living Rev. Relativity, 2, lrr-1999-2 (1999). [DOI], [ADS], [arXiv:gr-qc/9909058]. URL (accessed 28 March 2014): http://www.livingreviews.org/lrr-1999-2. (Cited on pages 55 and 62.)

[220] Konopliv, A. S., Asmar, S. W., Folkner, W. M., Karatekin, Ö., Nunes, D. C., Smrekar, S. E., Yoder, C. F. and Zuber, M. T., "Mars high resolution gravity fields from MRO, Mars seasonal gravity, and other dynamical parameters", Icarus, 211, 401-428 (2011). [DOI], [ADS]. (Cited on pages 47 and 50.)

[221] Kopeikin, S. M., "Testing the relativistic effect of the propagation of gravity by very long baseline interferometry", Astrophys. J. Lett., 556, L1-L5 (2001). [DOI], [gr-qc/0105060]. (Cited on page 45.)

[222] Kopeikin, S. M., "The post-Newtonian treatment of the VLBI experiment on September 8, 2002", Phys. Lett. A, 312, 147-157 (2003). [DOI], [gr-qc/0212121]. (Cited on page 45.)

[223] Kopeikin, S. M., "The speed of gravity in general relativity and theoretical interpretation of the Jovian deflection experiment", Class. Quantum Grav., 21, 3251-3286 (2004). [DOI], [gr-qc/0310059]. (Cited on pages 45 and 46.)

[224] Kopeikin, S. M., "Comment on 'Model-dependence of Shapiro time delay and the 'speed of gravity/speed of light' controversy"', Class. Quantum Grav., 22, 5181 (2005). [DOI], [gr-qc/0501048]. (Cited on page 46.)

[225] Kopeikin, S. M., "Comments on 'On the Speed of Gravity and the Jupiter/quasar Measurement' by S. Samuel", Int. J. Mod. Phys. D, 15, 273-288 (2006). [DOI], [ADS], [gr-qc/0501001]. (Cited on page 46.)

[226] Kopeikin, S. M. and Fomalont, E. B., "General relativistic model for experimental measurement of the speed of propagation of gravity by VLBI", in Ros, E., Porcas, R. W., Lobanov, A. P. and Zensus, J. A., eds., Proceedings of the 6th European VLBI Network Symposium, June 25-28 2002, Bonn, Germany, pp. 49-52, (Max-Planck-Institut für Radioastronomie, Bonn, 2002). [gr-qc/0206022]. (Cited on page 45.)

[227] Kostelecký, V. A. and Lane, C. D., "Constraints on Lorentz violation from clock-comparison experiments", Phys. Rev. D, 60, 116010 (1999). [DOI], [arXiv:hep-ph/9908504]. (Cited on page 13.)

[228] Kostelecký, V. A. and Mewes, M., "Signals for Lorentz violation in electrodynamics", Phys. Rev. D, 66, 056005 (2002). [DOI], [arXiv:hep-ph/0205211]. (Cited on pages 13 and 24.)

[229] Kostelecký, V. A. and Russell, N., "Data tables for Lorentz and CPT violation", Rev. Mod. Phys., 83, 11-31 (2011). [DOI], [arXiv:0801.0287 [hep-ph]]. (Cited on pages 15 and 24.)

[230] Kostelecký, V. A. and Samuel, S., "Gravitational phenomenology in higher-dimensional theories and strings", Phys. Rev. D, 40, 1886-1903 (1989). [DOI]. (Cited on page 38.) 
[231] Kramer, M., "Determination of the geometry of the PSR B1913+16 system by geodetic precession", Astrophys. J., 509, 856-860 (1998). [DOI], [astro-ph/9808127]. (Cited on page 72.)

[232] Kramer, M. et al., "Tests of General Relativity from Timing the Double Pulsar", Science, 314, 97-102 (2006). [DOI], [ADS], [arXiv:astro-ph/0609417]. (Cited on page 72.)

[233] Krisher, T. P., Anderson, J. D. and Campbell, J. K., "Test of the gravitational redshift effect at Saturn", Phys. Rev. Lett., 64, 1322-1325 (1990). [DOI]. (Cited on page 17.)

[234] Krisher, T. P., Maleki, L., Lutes, G. F., Primas, L. E., Logan, R. T., Anderson, J. D. and Will, C. M., "Test of the isotropy of the one-way speed of light using hydrogen-maser frequency standards", Phys. Rev. D, 42, 731-734 (1990). [DOI]. (Cited on page 12.)

[235] Krisher, T. P., Morabito, D. D. and Anderson, J. D., "The Galileo solar redshift experiment", Phys. Rev. Lett., 70, 2213-2216 (1993). [DOI]. (Cited on page 17.)

[236] Królak, A., Kokkotas, K. D. and Schäfer, G., "Estimation of the post-Newtonian parameters in the gravitational-wave emission of a coalescing binary", Phys. Rev. D, 52, 2089-2111 (1995). [grqc/9503013]. (Cited on page 84.)

[237] Lambert, S. B. and Le Poncin-Lafitte, C., "Determining the relativistic parameter $\gamma$ using very long baseline interferometry", Astron. Astrophys., 499, 331-335 (2009). [DOI], [ADS], [arXiv:0903.1615 [gr-qc]]. (Cited on page 44.)

[238] Lambert, S. B. and Le Poncin-Lafitte, C., "Improved determination of $\gamma$ by VLBI", Astron. Astrophys., 529, A70 (2011). [DOI], [ADS]. (Cited on page 44.)

[239] Lämmerzahl, C., "The Einstein equivalence principle and the search for new physics", in Giulini, D. J. W., Kiefer, C. and Lämmerzahl, C., eds., Quantum Gravity: From Theory to Experimental Search, Lecture Notes in Physics, 631, pp. 367-394, (Springer, Berlin; New York, 2003). [Google Books]. (Cited on page 10.)

[240] Lamoreaux, S. K., Jacobs, J. P., Heckel, B. R., Raab, F. J. and Fortson, E. N., "New limits on spatial anisotropy from optically-pumped ${ }^{201} \mathrm{Hg}$ and ${ }^{199} \mathrm{Hg} "$, Phys. Rev. Lett., 57, 3125-3128 (1986). [DOI], [ADS]. (Cited on page 13.)

[241] Lang, R. N., "Compact binary systems in scalar-tensor gravity. II. Tensor gravitational waves to second post-Newtonian order", Phys. Rev. D, 89, 084014 (2014). [DOI], [ADS], [arXiv:1310.3320 [gr-qc]]. (Cited on pages 62 and 65.)

[242] Lazaridis, K. et al., "Generic tests of the existence of the gravitational dipole radiation and the variation of the gravitational constant", Mon. Not. R. Astron. Soc., 400, 805-814 (2009). [DOI], [ADS], [arXiv:0908.0285 [astro-ph.GA]]. (Cited on page 50.)

[243] Lebach, D. E., Corey, B. E., Shapiro, I. I., Ratner, M. I., Webber, J. C., Rogers, A. E. E., Davis, J. L. and Herring, T. A., "Measurement of the Solar Gravitational Deflection of Radio Waves Using Very-Long-Baseline Interferometry", Phys. Rev. Lett., 75, 1439-1442 (1995). [DOI]. (Cited on page 44.)

[244] Lee, K., Jenet, F. A., Price, R. H., Wex, N. and Kramer, M., "Detecting Massive Gravitons Using Pulsar Timing Arrays", Astrophys. J., 722, 1589-1597 (2010). [DOI], [ADS], [arXiv:1008.2561 [astroph.HE]]. (Cited on page 86.)

[245] Lee, K. J., Jenet, F. A. and Price, R. H., "Pulsar Timing as a Probe of Non-Einsteinian Polarizations of Gravitational Waves", Astrophys. J., 685, 1304-1319 (2008). [DOI], [ADS]. (Cited on page 83.)

[246] Leefer, N., Weber, C. T. M., Cingöz, A., Torgerson, J. R. and Budker, D., "New Limits on Variation of the Fine-Structure Constant Using Atomic Dysprosium", Phys. Rev. Lett., 111, 060801 (2013). [DOI], [ADS], [arXiv:1304.6940 [physics.atom-ph]]. (Cited on pages 17, 18, and 19.) 
[247] Lehner, L., "Numerical relativity: a review", Class. Quantum Grav., 18, R25-R86 (2001). [DOI], [gr-qc/0106072]. (Cited on page 55.)

[248] Lentati, L. et al., "Variations in the fundamental constants in the QSO host J1148+5251 at $z=6.4$ and the BR1202-0725 system at $z=4.7$ ", Mon. Not. R. Astron. Soc., 430, 2454-2463 (2013). [DOI], [ADS], [arXiv:1211.3316 [astro-ph.CO]]. (Cited on pages 18 and 19.)

[249] Levi-Civita, T., "Astronomical consequences of the relativistic two-body problem", Am. J. Math., 59, 225-334 (1937). [DOI]. (Cited on page 57.)

[250] Li, T. G. F. et al., "Towards a generic test of the strong field dynamics of general relativity using compact binary coalescence: Further investigations", J. Phys.: Conf. Ser., 363, 012028 (2012). [DOI], [ADS], [arXiv:1111.5274 [gr-qc]]. (Cited on page 85.)

[251] Liberati, S., "Tests of Lorentz invariance: a 2013 update", Class. Quantum Grav., 30, 133001 (2013). [DOI], [ADS], [arXiv:1304.5795 [gr-qc]]. (Cited on pages 15 and 24.)

[252] Lightman, A. P. and Lee, D. L., "Restricted proof that the weak equivalence principle implies the Einstein equivalence principle", Phys. Rev. D, 8, 364-376 (1973). [DOI]. (Cited on page 20.)

[253] Lineweaver, C. H., Tenorio, L., Smoot, G. F., Keegstra, P., Banday, A. J. and Lubin, P., "The dipole observed in the COBE DMR 4 year data", Astrophys. J., 470, 38-42 (1996). [DOI]. (Cited on page 13.)

[254] Lipa, J. A., Nissen, J. A., Wang, S., Stricker, D. A. and Avaloff, D., "New limit on signals of Lorentz violation in electrodynamics", Phys. Rev. Lett., 90, 060403 (2003). [DOI], [physics/0302093]. (Cited on page 13.)

[255] Liu, K., Wex, N., Kramer, M., Cordes, J. M. and Lazio, T. J. W., "Prospects for probing the spacetime of Sgr A* with pulsars", Astrophys. J., 747, 1 (2012). [DOI], [ADS], [arXiv:1112.2151 [astro-ph.HE]]. (Cited on page 87.)

[256] Lobo, J. A., "Spherical GW detectors and geometry", in Coccia, E., Veneziano, G. and Pizzella, G., eds., Second Edoardo Amaldi Conference on Gravitational Waves, Proceedings of the conference, held at CERN, Switzerland, 1-4 July, 1997, Edoardo Amaldi Foundation Series, pp. 168-179, (World Scientific, Singapore, 1998). [ADS]. (Cited on page 82.)

[257] Long, J. C., Chan, H. W., Churnside, A. B., Gulbis, E. A., Varney, M. C. M. and Price, J. C., "Upper limits to submillimetre-range forces from extra space-time dimensions", Nature, 421, 922-925 (2003). [DOI], [hep-ph/0210004]. (Cited on page 27.)

[258] Long, J. C., Chan, H. W. and Price, J. C., "Experimental status of gravitational-strength forces in the sub-centimeter regime", Nucl. Phys. B, 539, 23-34 (1999). [DOI], [hep-ph/9805217]. (Cited on page 27.)

[259] LoPresto, J. C., Schrader, C. and Pierce, A. K., "Solar gravitational redshift from the infrared oxygen triplet", Astrophys. J., 376, 757-760 (1991). [DOI]. (Cited on page 16.)

[260] Lorentz, H. A. and Droste, J., "The motion of a system of bodies under the influence of their mutual attraction, according to Einstein's theory", in The Collected Papers of H.A. Lorentz, Vol. 5, pp. 330-355, (Nijhoff, The Hague, 1937). [DOI], Translated from Versl. K. Akad. Wetensch. Amsterdam, 26, 392, 1917. (Cited on page 57.)

[261] Lorimer, D. R., "Binary and Millisecond Pulsars", Living Rev. Relativity, 11, lrr-2008-8 (2008). [DOI], [ADS], [arXiv:0811.0762]. URL (accessed 28 March 2014):

http://www.livingreviews.org/lrr-2008-8. (Cited on page 68.) 
[262] Lucchesi, D. M. and Peron, R., "Accurate Measurement in the Field of the Earth of the GeneralRelativistic Precession of the LAGEOS II Pericenter and New Constraints on Non-Newtonian Gravity", Phys. Rev. Lett., 105, 231103 (2010). [DOI], [ADS], [arXiv:1106.2905 [gr-qc]]. (Cited on page 47.)

[263] Lucchesi, D. M. and Peron, R., "LAGEOS II pericenter general relativistic precession (1993-2005): Error budget and constraints in gravitational physics", Phys. Rev. D, 89, 082002 (2014). [DOI], [ADS]. (Cited on page 47.)

[264] Lynch, R. S. et al., "The Green Bank Telescope $350 \mathrm{MHz}$ drift-scan survey II: Data analysis and the timing of 10 new pulsars, including a relativistic binary", Astrophys. J., 763, 81 (2013). [DOI], [ADS], [arXiv:1209.4296 [astro-ph.HE]]. (Cited on page 73.)

[265] Lyne, A. G. et al., "A Double-Pulsar System: A Rare Laboratory for Relativistic Gravity and Plasma Physics", Science, 303, 1153-1157 (2004). [DOI], [arXiv:astro-ph/0401086]. (Cited on page 72.)

[266] Maeda, K.-I., "On time variation of fundamental constants in superstring theories", Mod. Phys. Lett. A, 3, 243-249 (1988). [DOI]. (Cited on page 37.)

[267] Maggiore, M. and Nicolis, A., "Detection strategies for scalar gravitational waves with interferometers and resonant spheres", Phys. Rev. D, 62, 024004 (2000). [DOI], [ADS], [arXiv:gr-qc/9907055]. (Cited on page 82.)

[268] Magueijo, J., "New varying speed of light theories", Rep. Prog. Phys., 66, 2025-2068 (2003). [DOI], [arXiv:astro-ph/0305457]. (Cited on page 26.)

[269] Malaney, R. A. and Mathews, G. J., "Probing the early universe: A review of primordial nucleosynthesis beyond the standard big bang", Phys. Rep., 229, 147-219 (1993). [DOI]. (Cited on page 19.)

[270] Maleki, L. and Prestage, J. D., "SpaceTime Mission: Clock test of relativity at four solar radii", in Lämmerzahl, C., Everitt, C. W. F. and Hehl, F. W., eds., Gyros, Clocks, and Interferometers...: Testing Relativistic Gravity in Space, Proceedings of a meeting held in Bad Honnef, Germany, August $21-27,1999$, Lecture Notes in Physics, 562, p. 369, (Springer, Berlin; New York, 2001). (Cited on page 17.)

[271] Marion, H. et al., "A search for variations of fundamental constants using atomic fountain clock", Phys. Rev. Lett., 90, 150801 (2003). [DOI], [physics/0212112]. (Cited on page 18.)

[272] Marka, Z. and Marka, S., "Selected articles from 'The 8th Edoardo Amaldi Conference on Gravitational Waves (Amaldi 8)', Columbia University, New York, 22 -26 June 2009", Class. Quantum Grav., 27, 080301 (2010). [DOI]. (Cited on page 79.)

[273] Mattingly, D., "Modern Tests of Lorentz Invariance", Living Rev. Relativity, 8, lrr-2005-5 (2005). [DOI], [ADS], [arXiv:gr-qc/0502097]. URL (accessed 28 March 2014): http://www.livingreviews.org/lrr-2005-5. (Cited on pages 15 and 24.)

[274] Mattingly, D. and Jacobson, T. A., "Relativistic Gravity with a Dynamical Preferred Frame", in Kostelecký, V. A., ed., CPT and Lorentz Symmetry II, Proceedings of the Second Meeting, held at Indiana University, Bloomington, August 15-18, 2001, pp. 331-335, (World Scientific, Singapore; River Edge, 2002). [gr-qc/0112012]. (Cited on page 38.)

[275] Mecheri, R., Abdelatif, T., Irbah, A., Provost, J. and Berthomieu, G., "New values of gravitational moments $J_{2}$ and $J_{4}$ deduced from helioseismology", Solar Phys., 222, 191-197 (2004). [DOI], [ADS]. (Cited on page 47.)

[276] Mercuri, S. and Taveras, V., "Interaction of the Barbero-Immirzi field with matter and pseudoscalar perturbations", Phys. Rev. D, 80, 104007 (2009). [DOI], [ADS], [arXiv:0903.4407 [gr-qc]]. (Cited on page 41.) 
[277] Merkowitz, S. M., "Tests of Gravity Using Lunar Laser Ranging", Living Rev. Relativity, 13, lrr2010-7 (2010). [DOI], [ADS]. URL (accessed 28 March 2014):

http://www.livingreviews.org/lrr-2010-7. (Cited on page 48.)

[278] Merlet, S., Bodart, Q., Malossi, N., Landragin, A., Pereira Dos Santos, F., Gitlein, O. and Timmen, L., "Comparison between two mobile absolute gravimeters: optical versus atomic interferometers", Metrologia, 47, L9-L11 (2010). [DOI], [ADS], [arXiv:1005.0357 [physics.atom-ph]]. (Cited on page 12.)

[279] Michelson, A. A. and Morley, E. W., "On the Relative Motion of the Earth and the Luminiferous Ether", Am. J. Sci., 34, 333-345 (1887). [DOI]. Online version (accessed 28 March 2014):

http://www.aip.org/history/gap/Michelson/Michelson.html. (Cited on page 12.)

[280] "MICROSCOPE (MICRO-Satellite à traînée Compensée pour l'Observation du Principe d'Equivalence)", project homepage, CNES. URL (accessed 28 March 2014): http://smsc.cnes.fr/MICROSCOPE/. (Cited on page 12.)

[281] Mignard, F., "Fundamental Physics with GAIA", in Bienaymé, O. and Turon, C., eds., GAIA: A European Space Project, Summer School, held in Les Houches, France, 14-18 May 2001, EAS Publications Series, 2, pp. 107-121, (EDP Sciences, Les Ulis, 2002). [DOI], [ADS]. (Cited on page 54.)

[282] Milani, A., Vokrouhlický, D., Villani, D., Bonanno, C. and Rossi, A., "Testing general relativity with the BepiColombo radio science experiment", Phys. Rev. D, 66, 082001 (2002). [DOI]. (Cited on page 54.)

[283] Milgrom, M., "A modification of the Newtonian dynamics as a possible alternative to the hidden mass hypothesis", Astrophys. J., 270, 365-370 (1983). [DOI], [ADS]. (Cited on page 39.)

[284] Milgrom, M., "MOND effects in the inner Solar system", Mon. Not. R. Astron. Soc., 399, 474-486 (2009). [DOI], [ADS], [arXiv:0906.4817 [astro-ph.CO]]. (Cited on page 40.)

[285] Mino, Y., Sasaki, M., Shibata, M., Tagoshi, H. and Tanaka, T., "Black Hole Perturbation", Prog. Theor. Phys. Suppl., 128, 1-121 (1997). [DOI], [gr-qc/9712057]. (Cited on pages 55 and 62.)

[286] Mirshekari, S. and Will, C. M., "Compact binary systems in scalar-tensor gravity: Equations of motion to 2.5 post-Newtonian order", Phys. Rev. D, 87, 084070 (2013). [DOI], [ADS], [arXiv:1301.4680 [gr-qc]]. (Cited on pages 62, 63, 64, 66, 67, and 75.)

[287] Mirshekari, S., Yunes, N. and Will, C. M., "Constraining Lorentz-violating, modified dispersion relations with gravitational waves", Phys. Rev. D, 85, 024041 (2012). [DOI], [ADS], [arXiv:1110.2720 [gr-qc]]. (Cited on page 86.)

[288] Mishra, C. K., Arun, K. G., Iyer, B. R. and Sathyaprakash, B. S., "Parametrized tests of postNewtonian theory using Advanced LIGO and Einstein Telescope", Phys. Rev. D, 82, 064010 (2010). [DOI], [ADS], [arXiv:1005.0304 [gr-qc]]. (Cited on page 85.)

[289] Misner, C. W., Thorne, K. S. and Wheeler, J. A., Gravitation, (W. H. Freeman, San Francisco, 1973). [ADS]. (Cited on pages 28 and 32.)

[290] Mitchell, T. and Will, C. M., "Post-Newtonian gravitational radiation and equations of motion via direct integration of the relaxed Einstein equations. V. Evidence for the strong equivalence principle to second post-Newtonian order", Phys. Rev. D, 75, 124025 (2007). [DOI], [ADS], [arXiv:0704.2243 [gr-qc]]. (Cited on page 60.)

[291] Modenini, D. and Tortora, P., "Pioneer 10 and 11 orbit determination analysis shows no discrepancy with Newton-Einstein's laws of gravity", arXiv, e-print, (2013). [ADS], [arXiv:1311.4978 [gr-qc]]. (Cited on page 27.) 
[292] Moura, F. and Schiappa, R., "Higher-derivative-corrected black holes: perturbative stability and absorption cross section in heterotic string theory", Class. Quantum Grav., 24, 361-386 (2007). [DOI], [ADS], [hep-th/0605001]. (Cited on page 41.)

[293] Müller, H., Herrmann, S., Braxmaier, C., Schiller, S. and Peters, A., "Modern MichelsonMorley experiment using cryogenic optical resonators", Phys. Rev. Lett., 91, 020401 (2003). [DOI], [physics/0305117]. (Cited on page 13.)

[294] Müller, H., Peters, A. and Chu, S., "A precision measurement of the gravitational redshift by the interference of matter waves", Nature, 463, 926-929 (2010). [DOI], [ADS]. (Cited on page 12.)

[295] Müller, J., Schneider, M., Nordtvedt Jr, K. L. and Vokrouhlický, D., "What can LLR provide to relativity?", in Piran, T., ed., The Eighth Marcel Grossmann Meeting on Recent Developments in Theoretical and Experimental General Relativity, Gravitation and Relativistic Field Theories, Proceedings of the meeting held at the Hebrew University of Jerusalem, June 22-27, 1997, pp. 1151-1153, (World Scientific, Singapore, 1999). (Cited on pages 48 and 50.)

[296] Murphy, M. T., Webb, J. K., Flambaum, V. V., Dzuba, V. A., Churchill, C. W., Prochaska, J. X., Barrow, J. D. and Wolfe, A. M., "Possible evidence for a variable fine-structure constant from QSO absorption lines: motivations, analysis and results", Mon. Not. R. Astron. Soc., 327, 1208-1222 (2001). [DOI], [astro-ph/0012419]. (Cited on pages 18 and 19.)

[297] Murphy, T. W. et al., "Laser ranging to the lost Lunokhod 1 reflector", Icarus, 211, 1103-1108 (2011). [DOI], [ADS], [arXiv:1009.5720 [astro-ph.EP]]. (Cited on page 49.)

[298] Murphy Jr, T. W., Adelberger, E. G., Battat, J. B. R., Hoyle, C. D., Johnson, N. H., McMillan, R. J., Stubbs, C. W. and Swanson, H. E., "APOLLO: millimeter lunar laser ranging", Class. Quantum Grav., 29, 184005 (2012). [DOI], [ADS]. (Cited on page 49.)

[299] Narayan, R. and McClintock, J. E., "Advection-dominated accretion and the black hole event horizon", New Astron. Rev., 51, 733-751 (2008). [DOI], [ADS], [arXiv:0803.0322]. (Cited on page 87.)

[300] Ni, W.-T., "Equivalence principles and electromagnetism", Phys. Rev. Lett., 38, 301-304 (1977). [DOI]. (Cited on pages 20 and 24.)

[301] Nishizawa, A., Taruya, A., Hayama, K., Kawamura, S. and Sakagami, M.-A., "Probing nontensorial polarizations of stochastic gravitational-wave backgrounds with ground-based laser interferometers", Phys. Rev. D, 79, 082002 (2009). [DOI], [ADS], [arXiv:0903.0528 [astro-ph.CO]]. (Cited on page 82.)

[302] Nishizawa, A., Taruya, A. and Kawamura, S., "Cosmological test of gravity with polarizations of stochastic gravitational waves around 0.1-1 Hz", Phys. Rev. D, 81, 104043 (2010). [DOI], [ADS], [arXiv:0911.0525 [gr-qc]]. (Cited on page 83.)

[303] Nordström, G., "Zur Theorie der Gravitation vom Standpunkt des Relativitätsprinzips", Ann. Phys. (Leipzig), 42, 533-554 (1913). [DOI]. (Cited on page 30.)

[304] Nordtvedt Jr, K. L., "Equivalence Principle for Massive Bodies. I. Phenomenology", Phys. Rev., 169, 1014-1016 (1968). [DOI], [ADS]. (Cited on page 47.)

[305] Nordtvedt Jr, K. L., "Equivalence Principle for Massive Bodies. II. Theory", Phys. Rev., 169, 10171025 (1968). [DOI], [ADS]. (Cited on page 32.)

[306] Nordtvedt Jr, K. L., "Existence of the gravitomagnetic interaction", Int. J. Theor. Phys., 27, 13951404 (1988). [DOI]. (Cited on page 51.)

[307] Nordtvedt Jr, K. L., "Gravitomagnetic interaction and laser ranging to Earth satellites", Phys. Rev. Lett., 61, 2647-2649 (1988). [DOI]. (Cited on page 51.)

[308] Nordtvedt Jr, K. L., " $\dot{G} / G$ and a cosmological acceleration of gravitationally compact bodies", Phys. Rev. Lett., 65, 953-956 (1990). [DOI]. (Cited on page 50.) 
[309] Nordtvedt Jr, K. L., "The Relativistic Orbit Observables in Lunar Laser Ranging", Icarus, 114, 51-62 (1995). [DOI]. (Cited on pages 48 and 49.)

[310] Nordtvedt Jr, K. L., "Testing Newton's third law using lunar laser ranging", Class. Quantum Grav., 18, L133-L137 (2001). [DOI]. (Cited on page 54.)

[311] Ohanian, H. C., "Comment on the Schiff Conjecture", Phys. Rev. D, 10, 2041-2042 (1974). [DOI]. (Cited on page 20.)

[312] Olive, K. A., Pospelov, M., Qian, Y.-Z., Manhès, G., Vangioni-Flam, E., Coc, A. and Cassé, M., "Reexamination of the ${ }^{187}$ Re bound on the variation of fundamental couplings", Phys. Rev. D, 69, 027701 (2004). [DOI], [astro-ph/0309252]. (Cited on page 19.)

[313] Palenzuela, C., Barausse, E., Ponce, M. and Lehner, L., "Dynamical scalarization of neutron stars in scalar-tensor gravity theories", Phys. Rev. D, 89, 044024 (2014). [DOI], [ADS], [arXiv:1310.4481 [gr-qc]]. (Cited on page 37.)

[314] Pani, P. and Cardoso, V., "Are black holes in alternative theories serious astrophysical candidates? The case for Einstein-dilaton-Gauss-Bonnet black holes", Phys. Rev. D, 79, 084031 (2009). [DOI], [ADS], [arXiv:0902.1569 [gr-qc]]. (Cited on page 41.)

[315] Paolozzi, A. and Ciufolini, I., "LARES successfully launched in orbit: Satellite and mission description", Acta Astronaut., 91, 313-321 (2013). [DOI], [ADS], [arXiv:1305.6823 [astro-ph.IM]]. (Cited on page 52.)

[316] Pati, M. E. and Will, C. M., "Post-Newtonian gravitational radiation and equations of motion via direct integration of the relaxed Einstein equations: Foundations", Phys. Rev. D, 62, 124015 (2000). [DOI], [gr-qc/0007087]. (Cited on page 60.)

[317] Pati, M. E. and Will, C. M., "Post-Newtonian gravitational radiation and equations of motion via direct integration of the relaxed Einstein equations. II. Two-body equations of motion to second postNewtonian order, and radiation reaction to 3.5 post-Newtonian order", Phys. Rev. D, 65, 104008 (2002). [DOI], [ADS], [gr-qc/0201001]. (Cited on page 60.)

[318] Peik, E., Lipphardt, B., Schnatz, H., Schneider, T. and Tamm, C., "Limit on the Present Temporal Variation of the Fine Structure Constant", Phys. Rev. Lett., 93, 170801 (2004). [DOI], [physics/0402132]. (Cited on page 18.)

[319] Peil, S., Crane, S., Hanssen, J. L., Swanson, T. B. and Ekstrom, C. R., "Tests of local position invariance using continuously running atomic clocks", Phys. Rev. A, 87, 010102 (2013). [DOI], [ADS], [arXiv:1301.6145 [physics.atom-ph]]. (Cited on page 17.)

[320] Petrov, Y. V., Nazarov, A. I., Onegin, M. S., Petrov, V. Y. and Sakhnovsky, E. G., "Natural nuclear reactor at Oklo and variation of fundamental constants: Computation of neutronics of a fresh core", Phys. Rev. C, 74, 064610 (2006). [DOI], [ADS], [hep-ph/0506186]. (Cited on pages 18 and 19.)

[321] Pitjeva, E. V., "Relativistic Effects and Solar Oblateness from Radar Observations of Planets and Spacecraft", Astron. Lett., 31, 340-349 (2005). [DOI], [ADS]. (Cited on page 50.)

[322] Poisson, E., "Measuring black-hole parameters and testing general relativity using gravitational-wave data from space-based interferometers", Phys. Rev. D, 54, 5939-5953 (1996). [DOI], [gr-qc/9606024]. (Cited on page 84.)

[323] Poisson, E. and Will, C. M., "Gravitational waves from inspiraling compact binaries: Parameter estimation using second-post-Newtonian wave forms", Phys. Rev. D, 52, 848-855 (1995). [DOI], [arXiv:gr-qc/9502040]. (Cited on page 83.)

[324] Poisson, E. and Will, C. M., Gravity: Newtonian, Post-Newtonian, Relativistic, (Cambridge University Press, Cambridge, 2014). (Cited on pages 28, 35, 36, 59, 80, and 82.) 
[325] Prestage, J. D., Bollinger, J. J., Itano, W. M. and Wineland, D. J., "Limits for Spatial Anisotropy by Use of Nuclear-Spin-Polarized ${ }^{9} \mathrm{Be}^{+}$Ions", Phys. Rev. Lett., 54, 2387-2390 (1985). [DOI]. (Cited on page 13.)

[326] Prestage, J. D., Tjoelker, R. L. and Maleki, L., "Atomic clocks and variation of the fine structure constant", Phys. Rev. Lett., 74, 3511-3514 (1995). [DOI]. (Cited on page 17.)

[327] Psaltis, D., "Measurements of black hole spins and tests of strong-field general relativity", in Kaaret, P., Lamb, F. K. and Swank, J. H., eds., X-Ray Timing 2003: Rossi and Beyond, Proceedings of the conference held 3-5 November 2003 in Cambridge, MA, AIP Conference Proceedings, 714, pp. 29-35, (American Institute of Physics, Melville, NY, 2004). [astro-ph/0402213]. (Cited on page 87.)

[328] Psaltis, D., "Probes and Tests of Strong-Field Gravity with Observations in the Electromagnetic Spectrum", Living Rev. Relativity, 11, lrr-2008-9 (2008). [DOI], [ADS], [arXiv:0806.1531]. URL (accessed 28 March 2014):

http://www.livingreviews.org/lrr-2008-9. (Cited on page 87.)

[329] Quast, R., Reimers, D. and Levshakov, S. A., "Probing the variability of the fine-structure constant with the VLT/UVES", Astron. Astrophys., 415, L7-L11 (2004). [DOI], [astro-ph/0311280]. (Cited on pages 18 and 19.)

[330] Randall, L. and Sundrum, R., "An Alternative to Compactification", Phys. Rev. Lett., 83, 4690-4693 (1999). [DOI], [ADS], [arXiv:hep-th/9906064]. (Cited on page 27.)

[331] Randall, L. and Sundrum, R., "Large Mass Hierarchy from a Small Extra Dimension", Phys. Rev. Lett., 83, 3370-3373 (1999). [DOI], [hep-ph/9905221]. (Cited on page 27.)

[332] Ransom, S. M. et al., "A millisecond pulsar in a stellar triple system", Nature, 505, 520-524 (2014). [DOI], [ADS], [arXiv:1401.0535 [astro-ph.SR]]. (Cited on pages 12, 49, and 73.)

[333] Reasenberg, R. D. et al., "Viking relativity experiment: Verification of signal retardation by solar gravity", Astrophys. J. Lett., 234, L219-L221 (1979). [DOI], [ADS]. (Cited on page 45.)

[334] Reeves, H., "On the origin of the light elements $(Z<6)$ ", Rev. Mod. Phys., 66, 193-216 (1994). [DOI]. (Cited on page 19.)

[335] Reynaud, S., Salomon, C. and Wolf, P., "Testing General Relativity with Atomic Clocks", Space Sci. Rev., 148, 233-247 (2009). [DOI], [ADS], [arXiv:0903.1166 [quant-ph]]. (Cited on page 17.)

[336] Reynolds, C. S., "Measuring Black Hole Spin Using X-Ray Reflection Spectroscopy", Space Sci. Rev. (2013). [DOI], [ADS], [arXiv:1302.3260 [astro-ph.HE]]. (Cited on page 87.)

[337] Reynolds, C. S., "The spin of supermassive black holes", Class. Quantum Grav., 30, 244004 (2013). [DOI], [ADS], [arXiv:1307.3246 [astro-ph.HE]]. (Cited on page 87.)

[338] Ries, J. C., Eanes, R. J., Tapley, B. D. and Peterson, G. E., "Prospects for an Improved LenseThirring Test with SLR and the GRACE Gravity Mission", in Noomen, R., Klosko, S., Noll, C. and Pearlman, M., eds., 13th International Workshop on Laser Ranging, Proceedings from the Science Session and Full Proceedings CD-ROM, Washington, DC, October 07-11, 2002, NASA Conference Proceedings, 2003-212248, pp. 211-248. NASA, (2003). URL (accessed 28 March 2014): http://cddis.gsfc.nasa.gov/lw13/1w_proceedings.html. (Cited on page 52.)

[339] Rievers, B. and Lämmerzahl, C., "High precision thermal modeling of complex systems with application to the flyby and Pioneer anomaly", Ann. Phys. (Berlin), 523, 439-449 (2011). [DOI], [ADS], [arXiv:1104.3985 [gr-qc]]. (Cited on page 27.)

[340] Riis, E., Anderson, L.-U. A., Bjerre, N., Poulson, O., Lee, S. A. and Hall, J. L., "Test of the Isotropy of the Speed of Light Using Fast-Beam Laser Spectroscopy", Phys. Rev. Lett., 60, 81-84 (1988). [DOI]. (Cited on page 12.) 
[341] Robertson, H. P., "The two-body problem in general relativity.", Ann. Math., 39, 101-104 (1938). [DOI]. (Cited on page 57.)

[342] Roll, P. G., Krotkov, R. and Dicke, R. H., "The equivalence of inertial and passive gravitational mass", Ann. Phys. (N.Y.), 26, 442-517 (1964). [DOI]. (Cited on page 47.)

[343] Rossi, B. and Hall, D. B., "Variation of the rate of decay of mesotrons with momentum", Phys. Rev., 59, 223-228 (1941). [DOI]. (Cited on page 12.)

[344] Rozelot, J.-P. and Damiani, C., "History of solar oblateness measurements and interpretation", Eur. Phys. J. H, 36, 407-436 (2011). [DOI], [ADS]. (Cited on page 47.)

[345] Ryan, F. D., "Gravitational waves from the inspiral of a compact object into a massive, axisymmetric body with arbitrary multipole moments", Phys. Rev. D, 52, 5707-5718 (1995). [DOI], [ADS]. (Cited on page 84.)

[346] Sagi, E., "Preferred frame parameters in the tensor-vector-scalar theory of gravity and its generalization", Phys. Rev. D, 80, 044032 (2009). [DOI], [ADS], [0905.4001 [gr-qc]]. (Cited on page 39.)

[347] Sampson, L., Yunes, N. and Cornish, N., "Rosetta stone for parametrized tests of gravity", Phys. Rev. D, 88, 064056 (2013). [DOI], [ADS], [arXiv:1307.8144 [gr-qc]]. (Cited on page 85.)

[348] Samuel, S., "On the speed of gravity and the v/c corrections to the Shapiro time delay", Phys. Rev. Lett., 90, 231101 (2003). [DOI], [astro-ph/0304006]. (Cited on page 45.)

[349] Samuel, S., "On the Speed of Gravity and the Jupiter/quasar Measurement", Int. J. Mod. Phys. D, 13, 1753-1770 (2004). [DOI], [astro-ph/0412401]. (Cited on page 45.)

[350] Santiago, D. I., Kalligas, D. and Wagoner, R. V., "Nucleosynthesis constraints on scalar-tensor theories of gravity", Phys. Rev. D, 56, 7627-7637 (1997). [DOI]. (Cited on page 87.)

[351] Sasaki, M. and Tagoshi, H., "Analytic Black Hole Perturbation Approach to Gravitational Radiation", Living Rev. Relativity, 6, lrr-2003-6 (2003). [DOI], [ADS], [arXiv:gr-qc/0306120]. URL (accessed 28 March 2014):

http://www.livingreviews.org/lrr-2003-6. (Cited on pages 55 and 62.)

[352] Sathyaprakash, B. S. and Schutz, B. F., "Physics, Astrophysics and Cosmology with Gravitational Waves", Living Rev. Relativity, 12, lrr-2009-2 (2009). [DOI], [ADS], [arXiv:0903.0338 [gr-qc]]. URL (accessed 28 March 2014):

http://www.livingreviews.org/lrr-2009-2. (Cited on pages 59 and 79.)

[353] Scharre, P. D. and Will, C. M., "Testing scalar-tensor gravity using space gravitational-wave interferometers", Phys. Rev. D, 65, 042002 (2002). [DOI], [ADS], [arXiv:gr-qc/0109044]. (Cited on page 84.)

[354] Schlamminger, S., Choi, K.-Y., Wagner, T. A., Gundlach, J. H. and Adelberger, E. G., "Test of the Equivalence Principle Using a Rotating Torsion Balance", Phys. Rev. Lett., 100, 041101 (2008). [DOI], [ADS], [arXiv:0712.0607 [gr-qc]]. (Cited on page 11.)

[355] Schutz, B. F., A First Course in General Relativity, (Cambridge University Press, Cambridge; New York, 2009), 2nd edition. [ADS], [Google Books]. (Cited on page 28.)

[356] Shah, A. G, "Gravitational-wave flux for a particle orbiting a Kerr black hole to 20th post-Newtonian order: a numerical approach", arXiv, e-print, (2014). [ADS], [arXiv:1403.2697]. (Cited on page 62.)

[357] Shankland, R. S., McCuskey, S. W., Leone, F. C. and Kuerti, G., "New analysis of the interferometer observations of Dayton C. Miller", Rev. Mod. Phys., 27, 167-178 (1955). [DOI]. (Cited on page 12.) 
[358] Shao, L., Caballero, R. N., Kramer, M., Wex, N., Champion, D. J. and Jessner, A., "A new limit on local Lorentz invariance violation of gravity from solitary pulsars", Class. Quantum Grav., 30, 165019 (2013). [DOI], [ADS], [arXiv:1307.2552 [gr-qc]]. (Cited on page 49.)

[359] Shao, L. and Wex, N., "New tests of local Lorentz invariance of gravity with small-eccentricity binary pulsars", Class. Quantum Grav., 29, 215018 (2012). [DOI], [ADS], [arXiv:1209.4503 [gr-qc]]. (Cited on page 49.)

[360] Shao, L. and Wex, N., "New limits on the violation of local position invariance of gravity", Class. Quantum Grav., 30, 165020 (2013). [DOI], [ADS], [arXiv:1307.2637 [gr-qc]]. (Cited on page 49.)

[361] Shapiro, I. I., "A century of relativity", Rev. Mod. Phys., 71, S41-S53 (1999). [DOI]. (Cited on page 8. .)

[362] Shapiro, I. I., Bartel, N., Bietenholz, M. F., Lebach, D. E., Lestrade, J.-F., Ransom, R. R. and Ratner, M. I., "VLBI for Gravity Probe B. I. Overview", Astrophys. J. Suppl. Ser., 201, 1 (2012). [DOI], [ADS], [arXiv:1204.4630 [astro-ph.IM]]. (Cited on page 52.)

[363] Shapiro, S. S., Davis, J. L., Lebach, D. E. and Gregory, J. S., "Measurement of the Solar Gravitational Deflection of Radio Waves using Geodetic Very-Long-Baseline Interferometry Data, 1979-1999", Phys. Rev. Lett., 92, 121101 (2004). [DOI]. (Cited on page 44.)

[364] Shibata, M., Taniguchi, K., Okawa, H. and Buonanno, A., "Coalescence of binary neutron stars in a scalar-tensor theory of gravity", Phys. Rev. D, 89, 084005 (2014). [DOI], [ADS], [arXiv:1310.0627 [gr-qc]]. (Cited on page 37.)

[365] Shlyakter, A. I., "Direct test of the constancy of fundamental nuclear constants", Nature, 264, 340 (1976). [DOI]. (Cited on page 18.)

[366] Skordis, C., "Generalizing tensor-vector-scalar cosmology", Phys. Rev. D, 77, 123502 (2008). [DOI], [ADS], [arXiv:0801.1985]. (Cited on page 39.)

[367] Skordis, C., "The tensor-vector-scalar theory and its cosmology", Class. Quantum Grav., 26, 143001 (2009). [DOI], [ADS], [arXiv:0903.3602 [astro-ph.CO]]. (Cited on page 40.)

[368] Smarr, L., Čadež, A., Dewitt, B. and Eppley, K., "Collision of two black holes: Theoretical framework", Phys. Rev. D, 14, 2443-2452 (1976). [DOI], [ADS]. (Cited on page 58.)

[369] Smiciklas, M., Brown, J. M., Cheuk, L. W., Smullin, S. J. and Romalis, M. V., "New Test of Local Lorentz Invariance Using a ${ }^{21} \mathrm{Ne}-\mathrm{Rb}-\mathrm{K}$ Comagnetometer", Phys. Rev. Lett., 107, 171604 (2011). [DOI], [ADS], [arXiv:1106.0738 [physics.atom-ph]]. (Cited on page 14.)

[370] Sotiriou, T. P. and Faraoni, V., " $f(R)$ theories of gravity", Rev. Mod. Phys., 82, 451-497 (2010) [DOI], [ADS], [arXiv:0805.1726 [gr-qc]]. (Cited on page 37.)

[371] Sotiriou, T. P. and Faraoni, V., "Black Holes in Scalar-Tensor Gravity", Phys. Rev. Lett., 108, 081103 (2012). [DOI], [ADS], [arXiv:1109.6324 [gr-qc]]. (Cited on page 67.)

[372] Speake, C. C. and Will, C. M., "Tests of the weak equivalence principle", Class. Quantum Grav., 29, 180301 (2012). [DOI]. (Cited on page 12.)

[373] Srianand, R., Chand, H., Petitjean, P. and Aracil, B., "Limits on the time variation of the electromagnetic fine-structure constant in the low energy limit from absorption lines in the spectra of distant quasars", Phys. Rev. Lett., 92, 121302 (2004). [DOI], [astro-ph/0402177]. (Cited on pages 18 and 19.)

[374] Stairs, I. H., "Testing General Relativity with Pulsar Timing", Living Rev. Relativity, 6, lrr-2003-5 (2003). [DOI], [ADS], [arXiv:astro-ph/0307536]. URL (accessed 28 March 2014):

http://www.livingreviews.org//rr-2003-5. (Cited on pages 49 and 68.) 
[375] Stairs, I. H. et al., "Discovery of three wide-orbit binary pulsars: Implications for Binary Evolution and Equivalence Principles", Astrophys. J., 632, 1060-1068 (2005). [DOI], [astro-ph/0506188]. (Cited on page 49.)

[376] Stanwix, P. L., Tobar, M. E., Wolf, P., Susli, M., Locke, C. R., Ivanov, E. N., Winterflood, J. and van Kann, F., "Test of Lorentz Invariance in Electrodynamics Using Rotating Cryogenic Sapphire Microwave Oscillators", Phys. Rev. Lett., 95, 040404 (2005). [DOI], [hep-ph/0506074]. (Cited on page 13.)

[377] Stavridis, A. and Will, C. M., "Bounding the mass of the graviton with gravitational waves: Effect of spin precessions in massive black hole binaries", Phys. Rev. D, 80, 044002 (2009). [DOI], [ADS], [arXiv:0906.3602 [gr-qc]]. (Cited on page 86.)

[378] Stecker, F. W. and Scully, S. T., "Searching for new physics with ultrahigh energy cosmic rays", New J. Phys., 11, 085003 (2009). [DOI], [ADS], [arXiv:0906.1735 [astro-ph.HE]]. (Cited on page 15.)

[379] Su, Y., Heckel, B. R., Adelberger, E. G., Gundlach, J. H., Harris, M., Smith, G. L. and Swanson, H. E., "New tests of the universality of free fall", Phys. Rev. D, 50, 3614-3636 (1994). [DOI]. (Cited on page 11.)

[380] Sushkov, A. O., Kim, W. J., Dalvit, D. A. R. and Lamoreaux, S. K., "New Experimental Limits on Non-Newtonian Forces in the Micrometer Range", Phys. Rev. Lett., 107, 171101 (2011). [DOI], [ADS], [arXiv:1108.2547 [quant-ph]]. (Cited on page 27.)

[381] Talmadge, C. L., Berthias, J.-P., Hellings, R. W. and Standish, E. M., "Model-Independent Constraints on Possible Modifications of Newtonian Gravity", Phys. Rev. Lett., 61, 1159-1162 (1988). [DOI], [ADS]. (Cited on pages 27 and 86.)

[382] Taveras, V. and Yunes, N., "Barbero-Immirzi parameter as a scalar field: K-inflation from loop quantum gravity?", Phys. Rev. D, 78, 064070 (2008). [DOI], [ADS], [arXiv:0807.2652 [gr-qc]]. (Cited on page 41.)

[383] Taylor, J. H., "Astronomical and Space Experiments to Test Relativity", in MacCallum, M. A. H., ed., General Relativity and Gravitation, p. 209, (Cambridge University Press, Cambridge; New York, 1987). [Google Books]. (Cited on page 17.)

[384] Taylor, T. R. and Veneziano, G., "Dilaton Couplings at Large Distances", Phys. Lett. B, 213, 450454 (1988). [DOI]. (Cited on pages 26 and 37.)

[385] Taylor Jr, J. H., "Nobel Lecture: Binary pulsars and relativistic gravity", Rev. Mod. Phys., 66, 711-719 (1994). [DOI]. (Cited on page 68.)

[386] Thorne, K. S., "Gravitational radiation", in Hawking, S. W. and Israel, W., eds., Three Hundred Years of Gravitation, pp. 330-458, (Cambridge University Press, Cambridge; New York, 1987). [Google Books]. (Cited on page 80.)

[387] Thorne, K. S. and Dykla, J. J., "Black Holes in the Dicke-Brans Theory of Gravity", Astrophys. J. Lett., 166, L35-L38 (1971). [DOI], [ADS]. (Cited on page 66.)

[388] Tinto, M. and Alves, M. E. D. S., "LISA sensitivities to gravitational waves from relativistic metric theories of gravity", Phys. Rev. D, 82, 122003 (2010). [DOI], [ADS], [arXiv:1010.1302 [gr-qc]]. (Cited on page 83.)

[389] Treuhaft, R. N. and Lowe, S. T., "A measurement of planetary relativistic deflection", Astron. J., 102, 1879-1888 (1991). [DOI]. (Cited on page 44.)

[390] Tu, L.-C., Guan, S.-G., Luo, J., Shao, C.-G. and Liu, L.-X., "Null Test of Newtonian Inverse-Square Law at Submillimeter Range with a Dual-Modulation Torsion Pendulum", Phys. Rev. Lett., 98, 201101 (2007). [DOI], [ADS]. (Cited on page 27.) 
[391] Turneaure, J. P., Will, C. M., Farrell, B. F., Mattison, E. M. and Vessot, R. F. C., "Test of the principle of equivalence by a null gravitational redshift experiment", Phys. Rev. D, 27, 1705-1714 (1983). [DOI]. (Cited on page 17.)

[392] Turyshev, S. G., "Experimental Tests of General Relativity", Annu. Rev. Nucl. Part. Sci., 58, 207248 (2008). [DOI], [ADS], [arXiv:0806.1731 [gr-qc]]. (Cited on page 8.)

[393] Turyshev, S. G., Shao, M. and Nordtvedt Jr, K. L., "Experimental design for the LATOR mission", Int. J. Mod. Phys. D, 13, 2035-2063 (2004). [DOI], [gr-qc/0410044]. (Cited on page 54.)

[394] Turyshev, S. G., Shao, M. and Nordtvedt Jr, K. L., "The laser astrometric test of relativity mission", Class. Quantum Grav., 21, 2773-2799 (2004). [DOI], [gr-qc/0311020]. (Cited on page 54.)

[395] Turyshev, S. G. and Toth, V. T., "The Pioneer Anomaly", Living Rev. Relativity, 13, lrr-2010-4 (2010). [DOI], [ADS], [arXiv:1001.3686 [gr-qc]]. URL (accessed 28 March 2014): http://www.livingreviews.org/lrr-2010-4. (Cited on page 27.)

[396] Turyshev, S. G., Toth, V. T., Kinsella, G., Lee, S.-C., Lok, S. M. and Ellis, J., "Support for the Thermal Origin of the Pioneer Anomaly", Phys. Rev. Lett., 108, 241101 (2012). [DOI], [ADS], [arXiv:1204.2507 [gr-qc]]. (Cited on page 27.)

[397] Uzan, J.-P., "Varying Constants, Gravitation and Cosmology", Living Rev. Relativity, 14, lrr-2011-2 (2011). [DOI], [ADS], [arXiv:1009.5514 [astro-ph.CO]]. URL (accessed 28 March 2014): http://www.livingreviews.org//rr-2011-2. (Cited on pages 18 and 19.)

[398] van Dam, H. and Veltman, M. J. G., "Massive and mass-less Yang-Mills and gravitational fields", Nucl. Phys. B, 22, 397-411 (1970). [DOI], [ADS]. (Cited on page 41.)

[399] Verma, A. K., Fienga, A., Laskar, J., Manche, H. and Gastineau, M., "Use of MESSENGER radioscience data to improve planetary ephemeris and to test general relativity", Astron. Astrophys., 561, A115 (2014). [DOI], [ADS], [arXiv:1306.5569 [astro-ph.EP]]. (Cited on page 47.)

[400] Vessot, R. F. C. et al., "Test of Relativistic Gravitation with a Space-Borne Hydrogen Maser", Phys. Rev. Lett., 45, 2081-2084 (1980). [DOI], [ADS]. (Cited on page 17.)

[401] Visser, M., "Mass for the graviton", Gen. Relativ. Gravit., 30, 1717-1728 (1998). [DOI], [arXiv:grqc/9705051]. (Cited on pages 29, 41, and 86.)

[402] Wagner, T. A., Schlamminger, S., Gundlach, J. H. and Adelberger, E. G., "Torsion-balance tests of the weak equivalence principle", Class. Quantum Grav., 29, 184002 (2012). [DOI], [ADS], [arXiv:1207.2442 [gr-qc]]. (Cited on page 11.)

[403] Wagoner, R. V., "Resonant-mass detection of tensor and scalar waves", in Marck, J.-A. and Lasota, J.-P., eds., Relativistic Gravitation and Gravitational Radiation, Proceedings of the Les Houches School of Physics, held in Les Houches, Haute Savoie, 26 September-6 October, 1995, pp. 419-432, (Cambridge University Press, Cambridge, 1997). [ADS]. (Cited on page 82.)

[404] Wagoner, R. V. and Kalligas, D., "Scalar-tensor theories and gravitational radiation", in Marck, J.-A. and Lasota, J.-P., eds., Relativistic Gravitation and Gravitational Radiation, Proceedings of the Les Houches School of Physics, held in Les Houches, Haute Savoie, 26 September-6 October, 1995, pp. 433-446, (Cambridge University Press, Cambridge, 1997). (Cited on page 82.)

[405] Wagoner, R. V. and Will, C. M., "Post-Newtonian gravitational radiation from orbiting point masses", Astrophys. J., 210, 764-775 (1976). [DOI]. (Cited on page 83.)

[406] Webb, J. K., Flambaum, V. V., Churchill, C. W., Drinkwater, M. J. and Barrow, J. D., "Search for time variation of the fine structure constant", Phys. Rev. Lett., 82, 884-887 (1999). [DOI], [astroph/9803165]. (Cited on pages 18 and 19.) 
[407] Weinberg, S., Gravitation and Cosmology: Principles and Applications of the General Theory of Relativity, (Wiley, New York, 1972). (Cited on page 28.)

[408] Weinberg, S., "Effective field theory for inflation", Phys. Rev. D, 77, 123541 (2008). [DOI], [ADS], [arXiv:0804.4291 [hep-th]]. (Cited on page 41.)

[409] Weisberg, J. M., Nice, D. J. and Taylor, J. H., "Timing Measurements of the Relativistic Binary Pulsar PSR B1913+16", Astrophys. J., 722, 1030-1034 (2010). [DOI], [ADS], [arXiv:1011.0718 [astroph.GA]]. (Cited on pages 68 and 71.)

[410] Weisberg, J. M. and Taylor, J. H., "General Relativistic Geodetic Spin Precession in Binary Pulsar B1913+16: Mapping the Emission Beam in Two Dimensions", Astrophys. J., 576, 942-949 (2002). [DOI], [astro-ph/0205280]. (Cited on page 72.)

[411] Wen, L. and Schutz, B. F., "Coherent network detection of gravitational waves: the redundancy veto", Class. Quantum Grav., 22, S1321-S1336 (2005). [DOI], [gr-qc/0508042]. Proceedings of the 9th Gravitational Wave Data Analysis Workshop, Annecy, France, 15-18 December 2004. (Cited on page 82.)

[412] Wex, N., "Testing Relativistic Gravity with Radio Pulsars", arXiv, e-print, (2014). [ADS], [arXiv:1402.5594 [gr-qc]]. (Cited on page 68.)

[413] Will, C. M., "Theoretical Frameworks for Testing Relativistic Gravity. II. Parametrized PostNewtonian Hydrodynamics, and the Nordtvedt Effect", Astrophys. J., 163, 611-628 (1971). [DOI], [ADS]. (Cited on page 32.)

[414] Will, C. M., "Active mass in relativistic gravity: Theoretical interpretation of the Kreuzer experiment", Astrophys. J., 204, 224-234 (1976). [DOI]. (Cited on page 53.)

[415] Will, C. M., "Gravitational radiation from binary systems in alternative metric theories of gravity: Dipole radiation and the binary pulsar", Astrophys. J., 214, 826-839 (1977). [DOI], [ADS]. (Cited on pages 62 and 74 .)

[416] Will, C. M., "Henry Cavendish, Johann von Soldner, and the deflection of light", Am. J. Phys., 56, 413-415 (1988). [DOI]. (Cited on page 42.)

[417] Will, C. M., "Twilight time for the fifth force?", Sky and Telescope, 80, 472-479 (1990). (Cited on page 27.)

[418] Will, C. M., "Clock synchronization and isotropy of the one-way speed of light", Phys. Rev. D, 45, 403-411 (1992). [DOI]. (Cited on pages 23 and 24.)

[419] Will, C. M., "Is momentum conserved? A test in the binary system PSR 1913+16", Astrophys. J. Lett., 393, L59-L61 (1992). [DOI]. (Cited on page 54.)

[420] Will, C. M., Theory and Experiment in Gravitational Physics, (Cambridge University Press, Cambridge; New York, 1993), 2nd edition. [Google Books]. (Cited on pages 8, 10, 11, 15, 16, 18, 20, 22, $28,31,32,34,35,36,38,42,44,45,47,48,49,51,53,54,66,69,75,76,82,83,85$, and 87.)

[421] Will, C. M., Was Einstein Right?: Putting General Relativity to the Test, (Basic Books, New York, 1993), 2nd edition. (Cited on page 7.)

[422] Will, C. M., "Testing scalar-tensor gravity with gravitational-wave observations of inspiralling compact binaries", Phys. Rev. D, 50, 6058-6067 (1994). [DOI], [arXiv:gr-qc/9406022]. (Cited on page 84.)

[423] Will, C. M., "Bounding the mass of the graviton using gravitational-wave observations of inspiralling compact binaries", Phys. Rev. D, 57, 2061-2068 (1998). [DOI], [ADS], [arXiv:gr-qc/9709011]. (Cited on pages 85 and 86 .) 
[424] Will, C. M., "Einstein's relativity and everyday life", online resource, American Physical Society, (2000). URL (accessed 28 March 2014): http://www.physicscentral.com/writers/writers-00-2.html. (Cited on page 17.)

[425] Will, C. M., "Propagation speed of gravity and the relativistic time delay", Astrophys. J., 590, 683-690 (2003). [DOI], [astro-ph/0301145]. (Cited on pages 45 and 46.)

[426] Will, C. M., "Special Relativity: A Centenary Perspective", in Damour, T., Darrigol, O., Duplantier, B. and Rivasseau, V., eds., Einstein, 1905-2005: Poincaré Seminar 2005, Progres in Mathematical Physics, 47, pp. 33-58, (Birkäuser Verlag, Basel; Boston; Berlin, 2006). [DOI], [ADS], [Google Books]. (Cited on page 12.)

[427] Will, C. M., "Testing the General Relativistic 'No-Hair' Theorems Using the Galactic Center Black Hole Sagittarius A*", Astrophys. J. Lett., 674, L25-L28 (2008). [DOI], [ADS], [arXiv:0711.1677]. (Cited on page 87.)

[428] Will, C. M., "Resource Letter PTG-1: Precision Tests of Gravity", Am. J. Phys., 78, 1240-1247 (2010). [DOI], [ADS], [arXiv:1008.0296 [gr-qc]]. (Cited on page 8.)

[429] Will, C. M., "Finally, results from Gravity Probe B", Physics, 4, 43 (2011). [DOI], [ADS], [arXiv:1106.1198 [gr-qc]]. URL (accessed 28 March 2014): http://physics.aps.org/articles/v4/43. (Cited on page 52.)

[430] Will, C. M., "On the unreasonable effectiveness of the post-Newtonian approximation in gravitational physics", Proc. Natl. Acad. Sci. USA, 108, 5938-5945 (2011). [DOI], [ADS], [arXiv:1102.5192 [gr-qc]]. (Cited on page 57.)

[431] Will, C. M. and Nordtvedt Jr, K. L., "Conservation Laws and Preferred Frames in Relativistic Gravity. I. Preferred-Frame Theories and an Extended PPN Formalism", Astrophys. J., 177, 757774 (1972). [DOI], [ADS]. (Cited on pages 32 and 38.)

[432] Will, C. M. and Wiseman, A. G., "Gravitational radiation from compact binary systems: Gravitational waveforms and energy loss to second post-Newtonian order", Phys. Rev. D, 54, 4813-4848 (1996). [DOI], [gr-qc/9608012]. (Cited on page 60.)

[433] Will, C. M. and Yunes, N., "Testing alternative theories of gravity using LISA", Class. Quantum Grav., 21, 4367-4381 (2004). [DOI], [ADS], [arXiv:gr-qc/0403100]. (Cited on pages 84 and 86.)

[434] Will, C. M. and Zaglauer, H. W., "Gravitational radiation, close binary systems, and the BransDicke theory of gravity", Astrophys. J., 346, 366-377 (1989). [DOI], [ADS]. (Cited on pages 62 and 66.)

[435] Williams, J. G., Newhall, X. X. and Dickey, J. O., "Relativity parameters determined from lunar laser ranging", Phys. Rev. D, 53, 6730-6739 (1996). [DOI]. (Cited on pages 48, 50, and 53.)

[436] Williams, J. G., Turyshev, S. G. and Boggs, D. H., "Progress in Lunar Laser Ranging Tests of Relativistic Gravity", Phys. Rev. Lett., 93, 261101 (2004). [DOI], [ADS], [arXiv:gr-qc/0411113]. (Cited on pages 48, 50, and 53.)

[437] Williams, J. G., Turyshev, S. G. and Murphy Jr, T. W., "Improving LLR Tests of Gravitational Theory", Int. J. Mod. Phys. D, 13, 567-582 (2004). [DOI], [gr-qc/0311021]. (Cited on pages 48 and 54.)

[438] Wolf, P., Bize, S., Clairon, A., Luiten, A. N., Santarelli, G. and Tobar, M. E., "Tests of Lorentz invariance using a microwave resonator", Phys. Rev. Lett., 90, 060402 (2003). [DOI], [gr-qc/0210049]. (Cited on page 13.)

[439] Wolf, P., Blanchet, L., Bordé, C. J., Reynaud, S., Salomon, C. and Cohen-Tannoudji, C., "Does an atom interferometer test the gravitational redshift at the Compton frequency?", Class. Quantum Grav., 28, 145017 (2011). [DOI], [ADS], [arXiv:1012.1194 [gr-qc]]. (Cited on page 12.) 
[440] Wolf, P., Chapelet, F., Bize, S. and Clairon, A., "Cold Atom Clock Test of Lorentz Invariance in the Matter Sector", Phys. Rev. Lett., 96, 060801 (2006). [DOI], [ADS], [hep-ph/0601024]. (Cited on page 14.)

[441] Wolfe, A. M., Brown, R. L. and Roberts, M. S., "Limits on the Variation of Fundamental Atomic Quantities over Cosmic Time Scales", Phys. Rev. Lett., 37, 179-181 (1976). [DOI]. (Cited on page 18.)

[442] Yagi, K., Blas, D., Barausse, E. and Yunes, N., "Constraints on Einstein-Æther theory and Hořava gravity from binary pulsar observations", Phys. Rev. D, 89, 084067 (2014). [DOI], [ADS], [arXiv:1311.7144 [gr-qc]]. (Cited on page 78.)

[443] Yagi, K., Blas, D., Yunes, N. and Barausse, E., "Strong binary pulsar constraints on Lorentz violation in gravity", Phys. Rev. Lett., 112, 161101 (2014). [DOI], [ADS], [arXiv:1307.6219 [gr-qc]]. (Cited on page 78.)

[444] Yagi, K., Stein, L. C., Yunes, N. and Tanaka, T., "Isolated and binary neutron stars in dynamical Chern-Simons gravity", Phys. Rev. D, 87, 084058 (2013). [DOI], [ADS], [arXiv:1302.1918 [gr-qc]]. (Cited on page 41.)

[445] Yagi, K. and Tanaka, T., "Constraining alternative theories of gravity by gravitational waves from precessing eccentric compact binaries with LISA", Phys. Rev. D, 81, 064008 (2010). [DOI], [ADS], [arXiv:0906.4269 [gr-qc]]. (Cited on pages 84 and 86.)

[446] Yagi, K. and Tanaka, T., "DECIGO/BBO as a probe to constrain alternative theories of gravity", Prog. Theor. Phys., 123, 1069-1078 (2010). [DOI], [ADS], [arXiv:0908.3283 [gr-qc]]. (Cited on pages 84 and 86.)

[447] Yagi, K. and Yunes, N., "I-Love-Q relations in neutron stars and their applications to astrophysics, gravitational waves, and fundamental physics", Phys. Rev. D, 88, 023009 (2013). [DOI], [ADS], [arXiv:1303.1528 [gr-qc]]. (Cited on page 85.)

[448] Yagi, K. and Yunes, N., "I-Love-Q: Unexpected universal relations for neutron stars and quark stars", Science, 341, 365-368 (2013). [DOI], [ADS], [arXiv:1302.4499 [gr-qc]]. (Cited on page 85.)

[449] Yang, S.-Q., Zhan, B.-F., Wang, Q.-L., Shao, C.-G., Tu, L.-C., Tan, W.-H. and Luo, J., "Test of the Gravitational Inverse Square Law at Millimeter Ranges", Phys. Rev. Lett., 108, 081101 (2012). [DOI], [ADS]. (Cited on page 27.)

[450] Yunes, N., Pani, P. and Cardoso, V., "Gravitational waves from quasicircular extreme mass-ratio inspirals as probes of scalar-tensor theories", Phys. Rev. D, 85, 102003 (2012). [DOI], [ADS], [arXiv:1112.3351 [gr-qc]]. (Cited on page 67.)

[451] Yunes, N. and Pretorius, F., "Fundamental theoretical bias in gravitational wave astrophysics and the parametrized post-Einsteinian framework", Phys. Rev. D, 80, 122003 (2009). [DOI], [ADS], [arXiv:0909.3328 [gr-qc]]. (Cited on page 85.)

[452] Yunes, N. and Siemens, X., "Gravitational-Wave Tests of General Relativity with Ground-Based Detectors and Pulsar-Timing Arrays", Living Rev. Relativity, 16, lrr-2013-9 (2013). [DOI], [ADS], [arXiv:1304.3473 [gr-qc]]. URL (accessed 28 March 2014):

http://www.livingreviews.org/lrr-2013-9. (Cited on page 79.)

[453] Zakharov, V. I., "Linearized gravitation theory and the graviton mass", JETP Lett., 12, 312 (1970). [ADS]. (Cited on page 41.)

[454] Zuntz, J., Baker, T., Ferreira, P. G. and Skordis, C., "Ambiguous tests of general relativity on cosmological scales", J. Cosmol. Astropart. Phys., 6, 032 (2012). [DOI], [ADS], [arXiv:1110.3830 [astro-ph.CO]]. (Cited on page 88.) 